\title{
Divergent Construction of Benzothiophene-Fused N-Heterocycles via Stereotunable Three-Component Domino Reactions
}

\author{
Qingsong Deng ${ }^{[\mathrm{a}]},{\text { Aimin } \mathrm{Yu}^{[\mathrm{a}]} \text {, Lei Zhang }}^{[\mathrm{b}]}$, Xiangtai Meng*[a] \\ ${ }^{[a]}$ Tianjin Key Laboratory of Organic Solar Cells and Photochemical Conversion, Tianjin Key \\ Laboratory of Drug Targeting and Bioimaging, School of Chemistry \& Chemical Engineering, \\ Tianjin University of Technology, Tianjin 300384, P. R. China \\ ${ }^{[b]}$ Tianjin Engineering Technology Center of Chemical Wastewater Source Reduction and \\ Recycling, School of Science Tianjin Chengjian University, Tianjin 300384, P.R. China \\ E-mail: xtmeng@tjut.edu.cn
}

Table of contents

1) NMR spectra of all new compounds..............................

2) Computational details and archive entries..........................

3) X-ray data collection and structure determinations.....................S-87

4) X-ray crystal structures...............................................

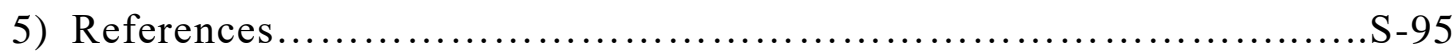




\section{NMR spectra of all new compounds}

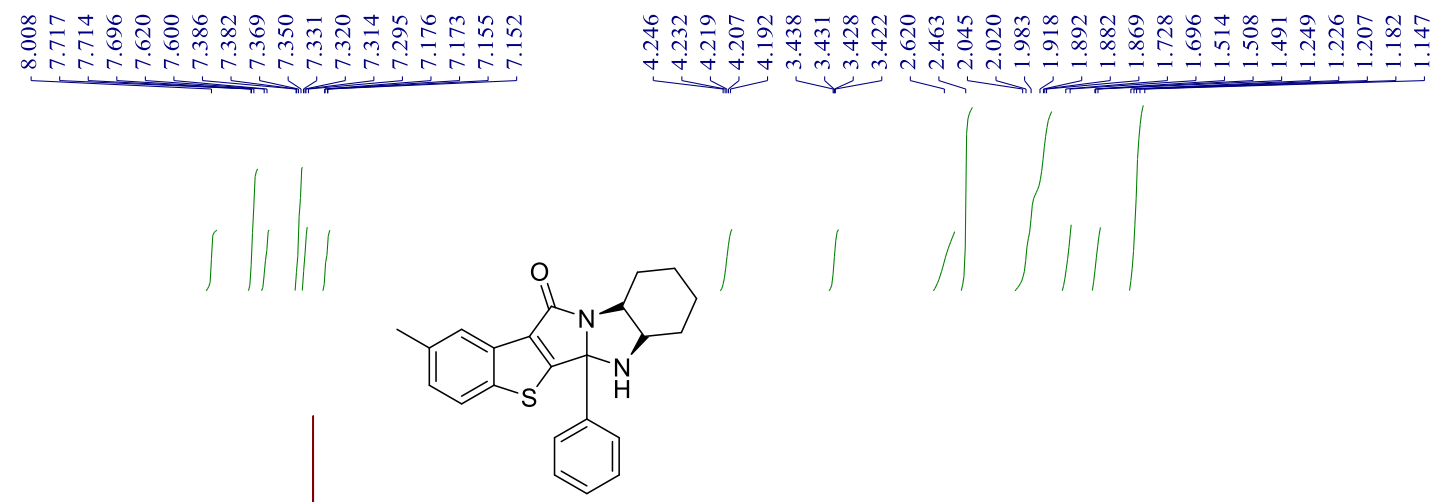

4a, ${ }^{1} \mathrm{H}$ NMR $400 \mathrm{MHz}, \mathrm{CDCl}_{3}$
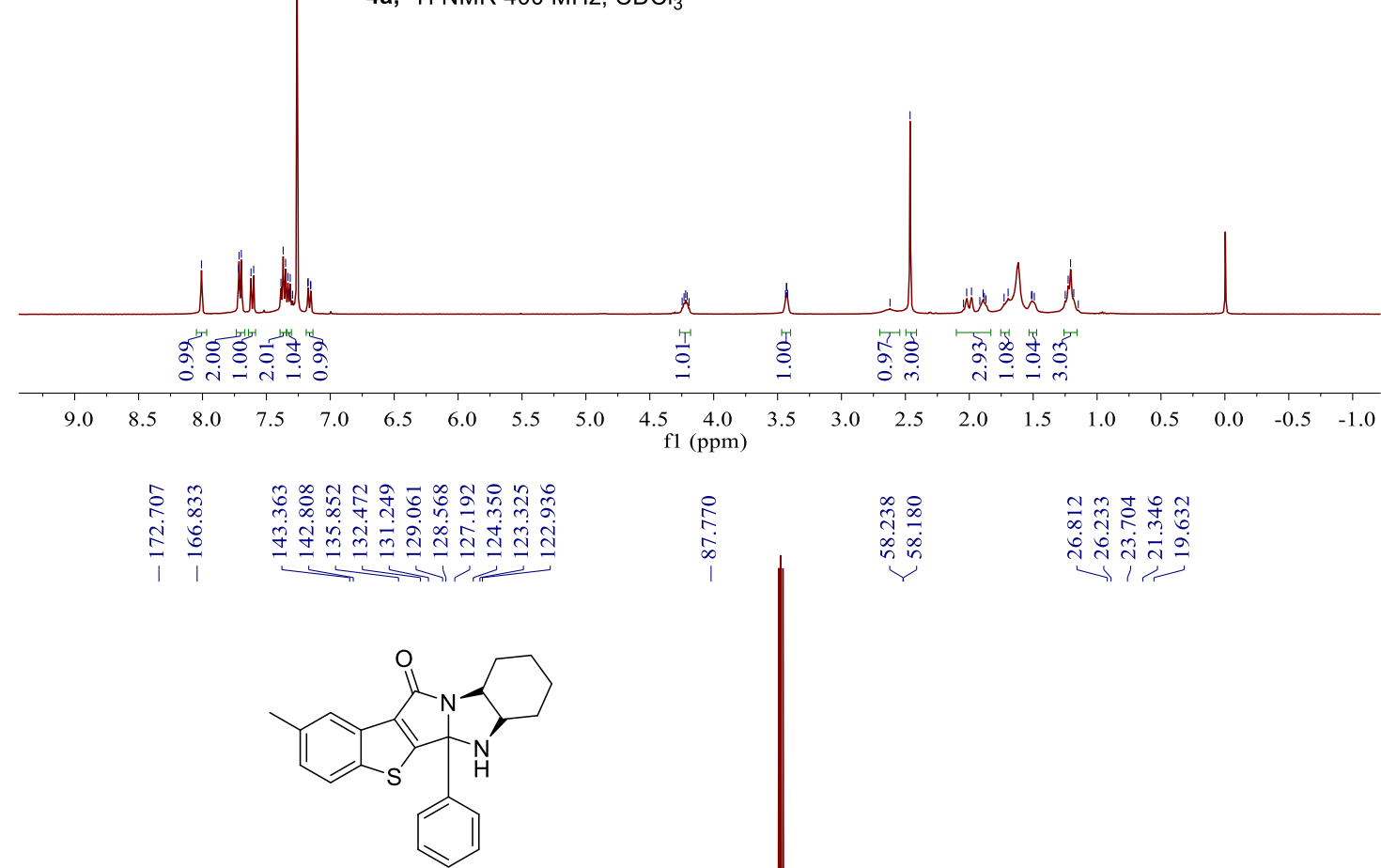

4a, ${ }^{13} \mathrm{C}$ NMR $100 \mathrm{MHz}, \mathrm{CDCl}_{3}$

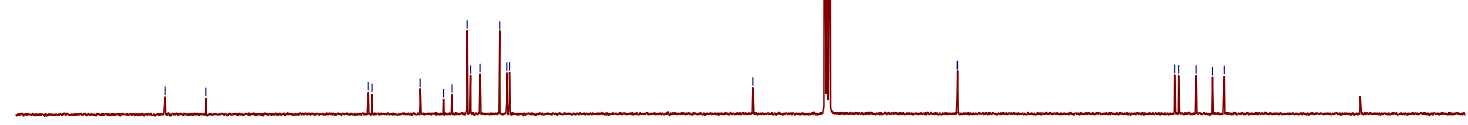

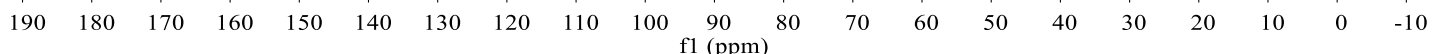




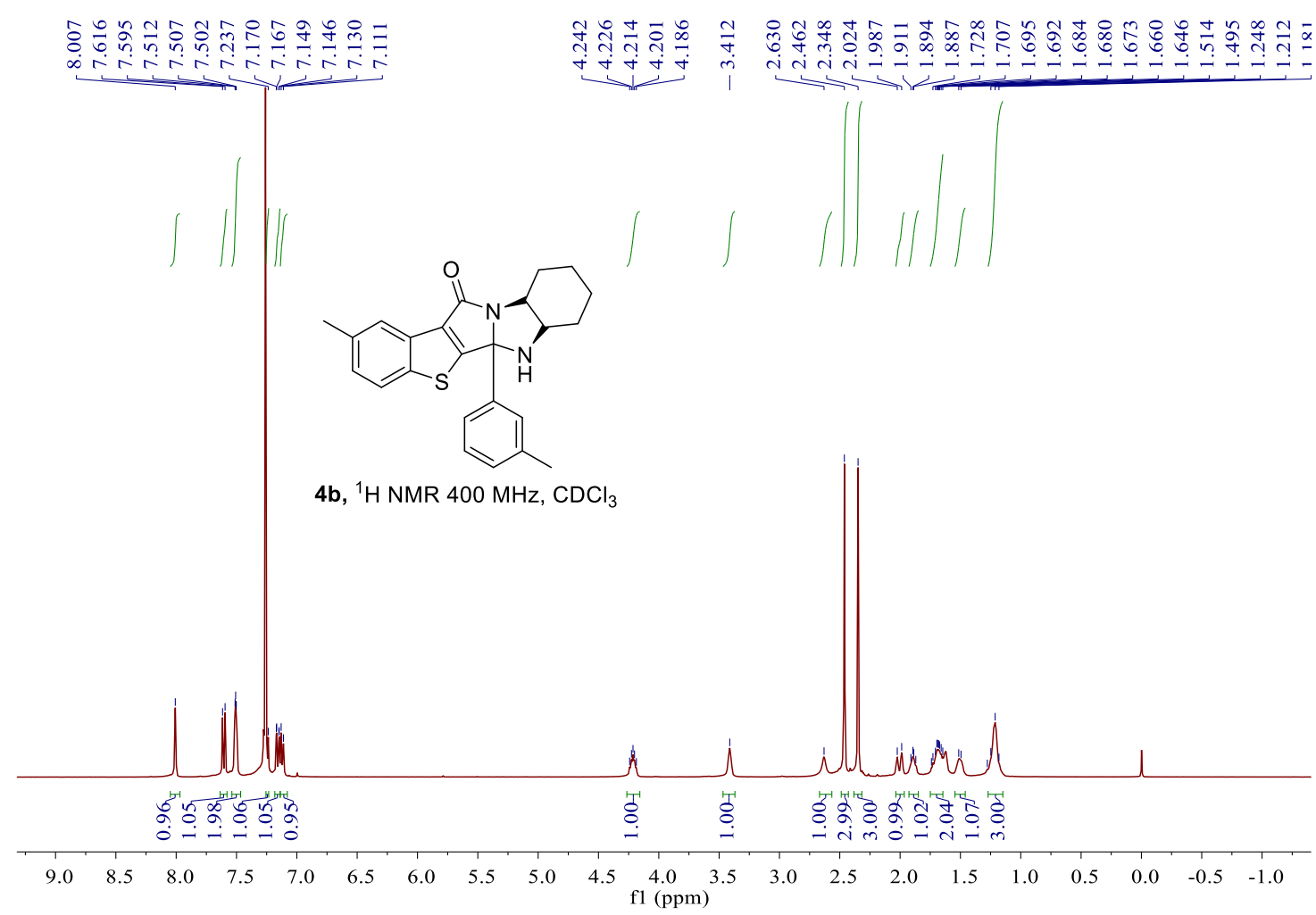

\begin{tabular}{|c|c|c|c|c|}
\hline 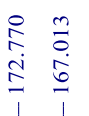 & 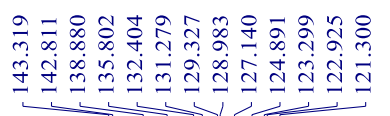 & $\begin{array}{l}\hat{N} \\
\infty \\
\infty \\
\infty \\
\infty\end{array}$ & 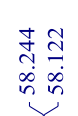 & 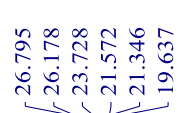 \\
\hline
\end{tabular}

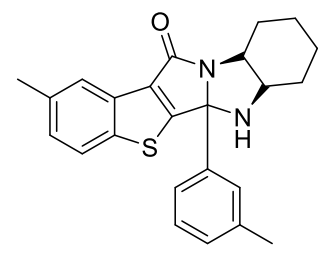

4b, ${ }^{13} \mathrm{C}$ NMR $100 \mathrm{MHz}, \mathrm{CDCl}_{3}$
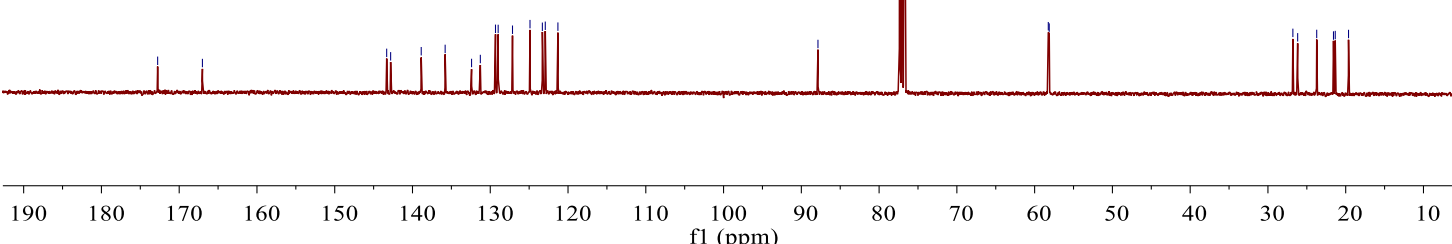

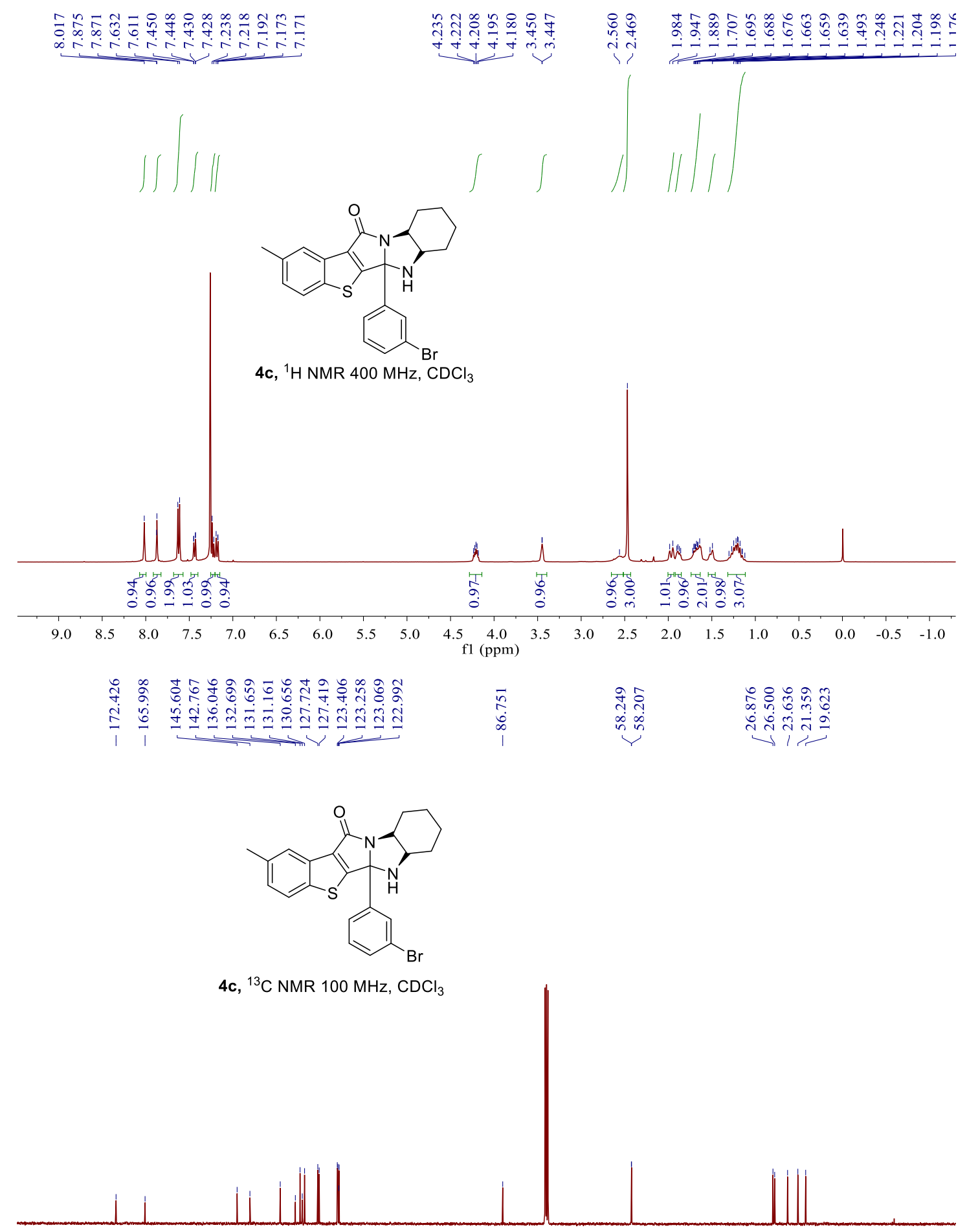

$\begin{array}{lllllllllllllllllllll}190 & 180 & 170 & 160 & 150 & 140 & 130 & 120 & 110 & 100 & \begin{array}{c}90 \\ \mathrm{fl} 1\end{array} & 80 & 70 & 60 & 50 & 40 & 30 & 20 & 10 & 0 & -10\end{array}$ 


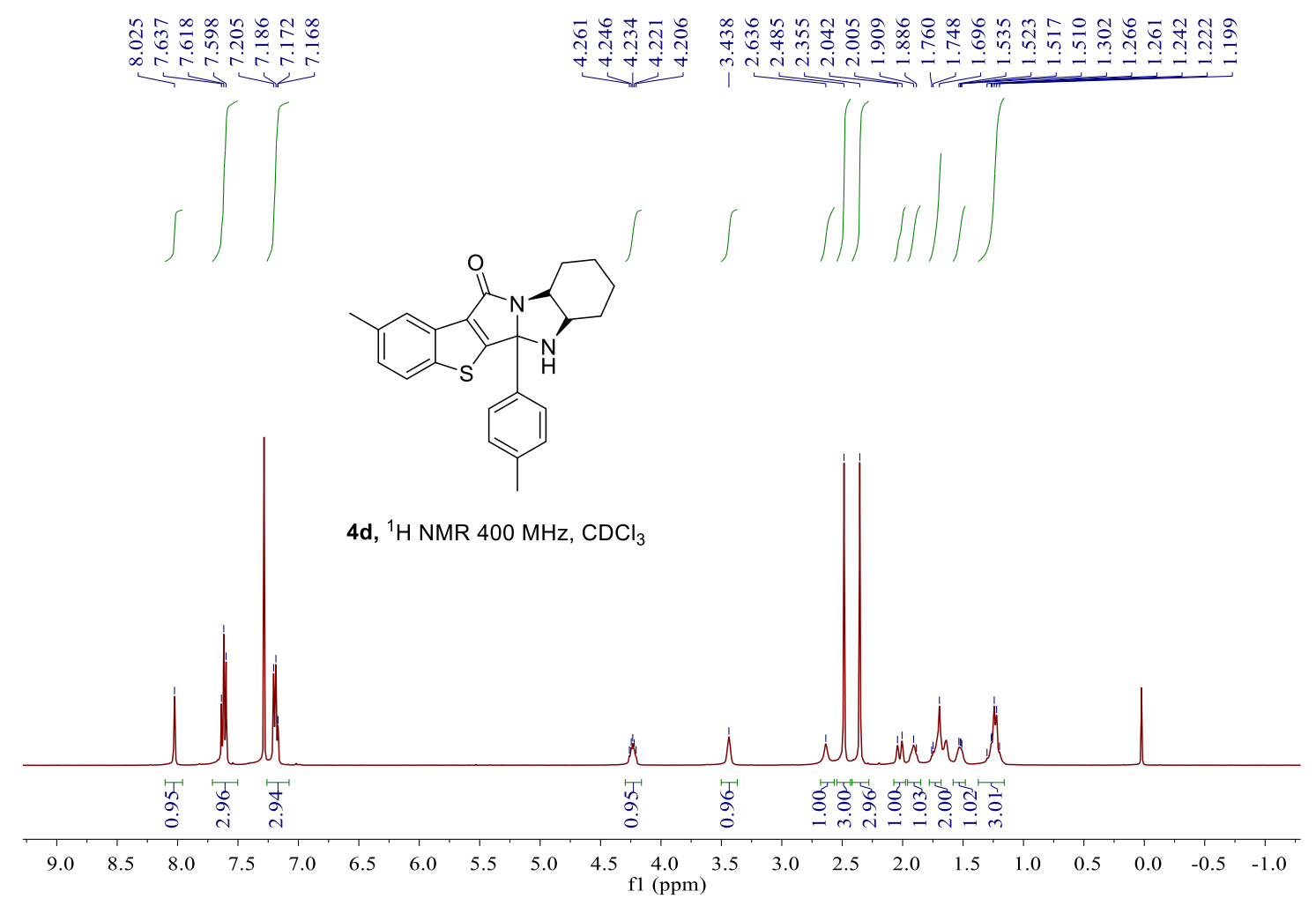

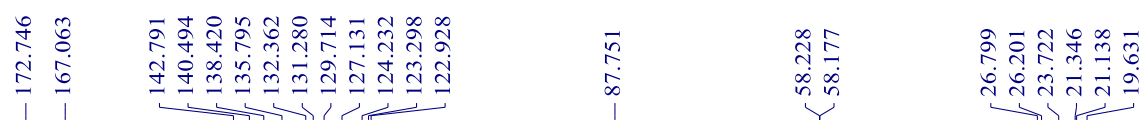

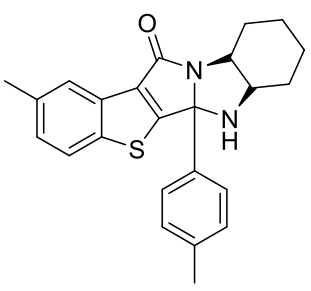

4d, ${ }^{13} \mathrm{C}$ NMR $100 \mathrm{MHz}, \mathrm{CDCl}_{3}$

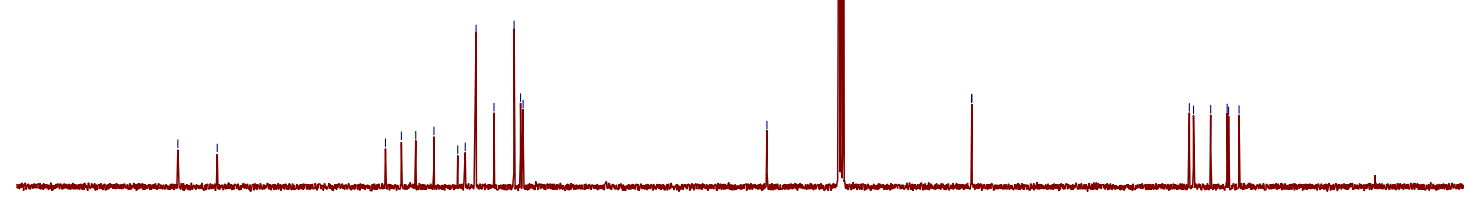

$\begin{array}{lllllllllllllllllllll}190 & 180 & 170 & 160 & 150 & 140 & 130 & 120 & 110 & 100 & 90 & 80 & 70 & 60 & 50 & 40 & 30 & 20 & 10 & 0 & -10\end{array}$ 

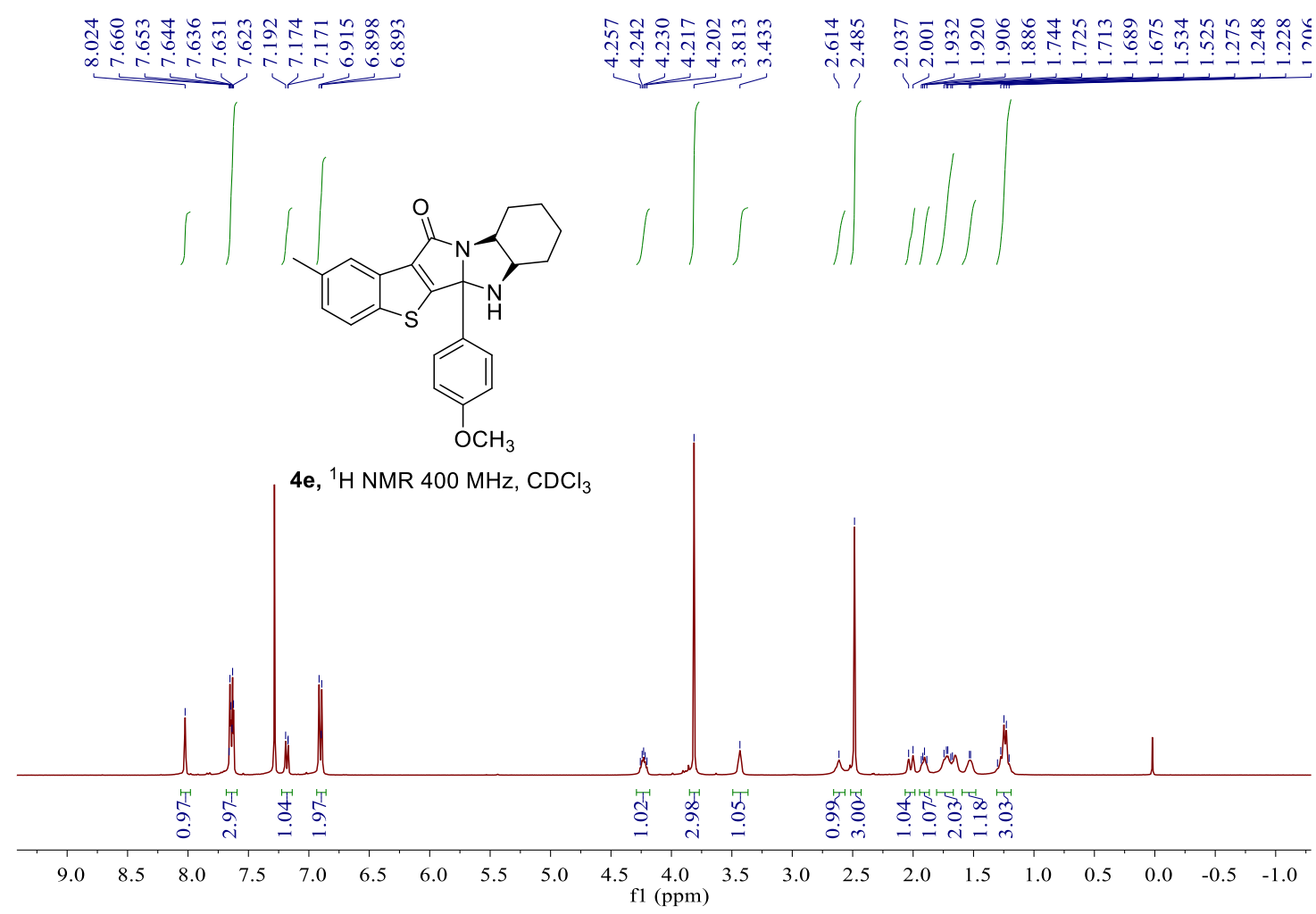

\begin{tabular}{|c|c|c|c|c|}
\hline 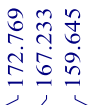 & 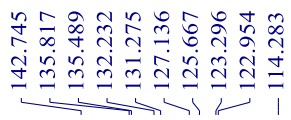 & $\underset{\substack{n \\
\infty \\
1}}{\infty}$ & 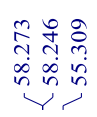 & 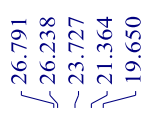 \\
\hline
\end{tabular}

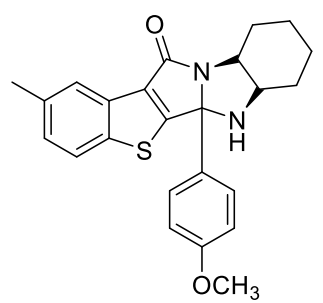

4e, ${ }^{13} \mathrm{C}$ NMR $100 \mathrm{MHz}, \mathrm{CDCl}_{3}$

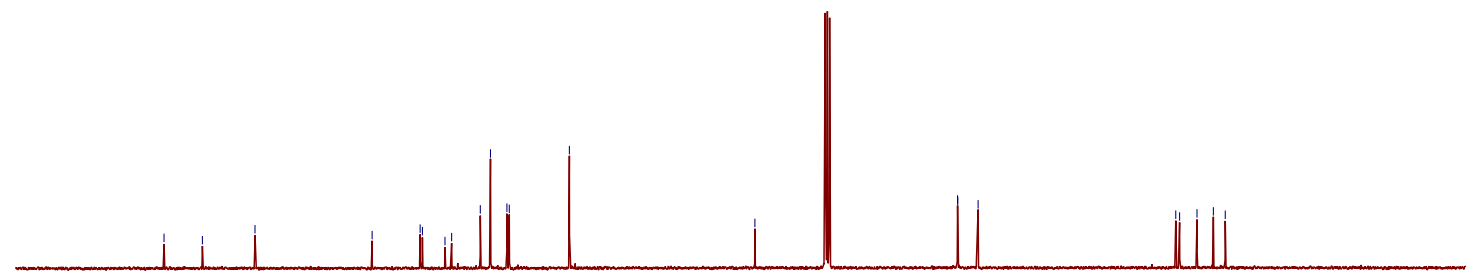

$\begin{array}{lllllllllllllllllllllllll}190 & 180 & 170 & 160 & 150 & 140 & 130 & 120 & 110 & 100 & 90 & 80 & 70 & 60 & 50 & 40 & 30 & 20 & 10 & 0 & -10\end{array}$ 

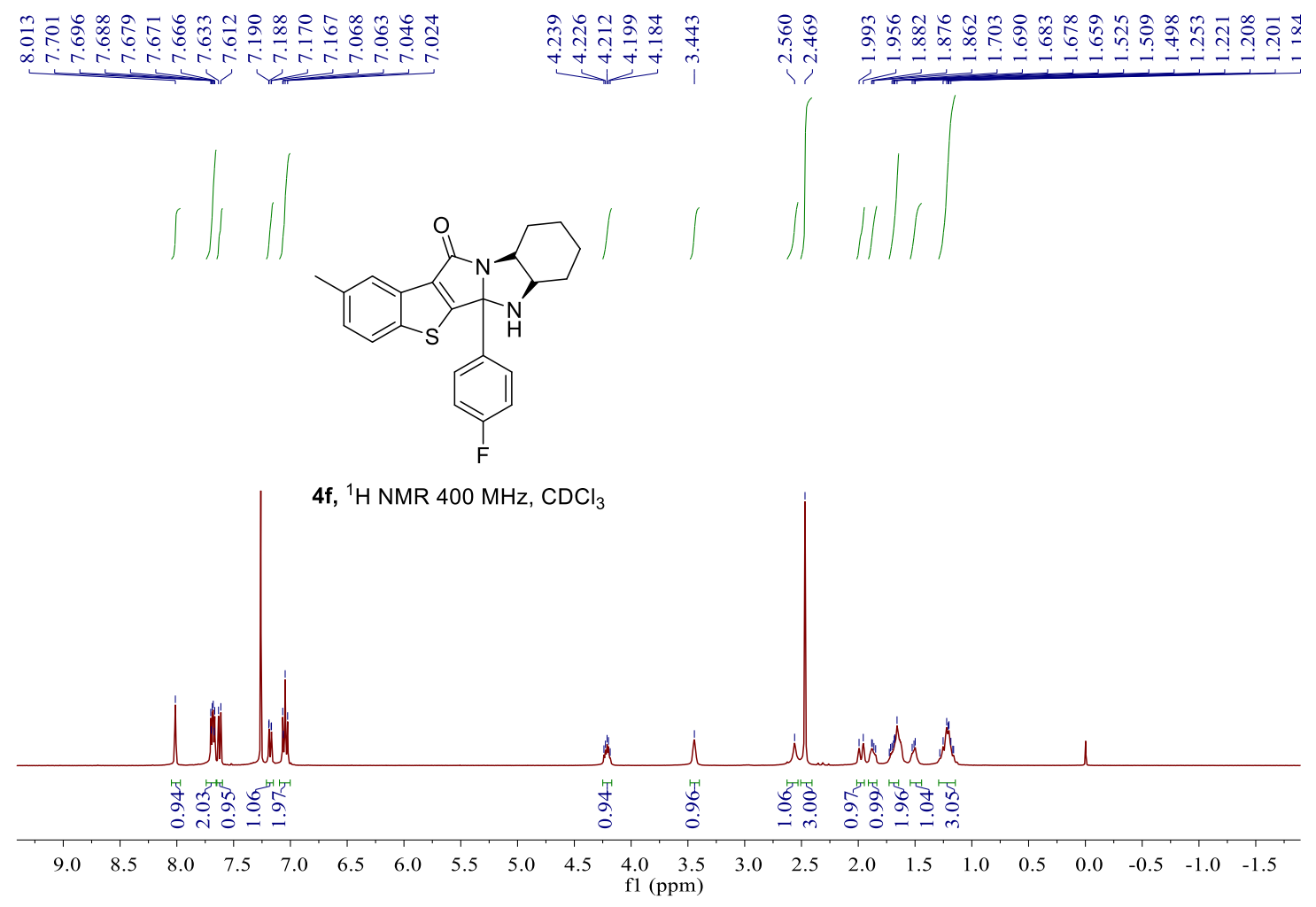

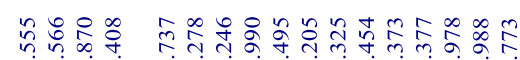

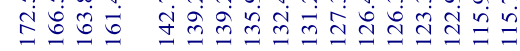

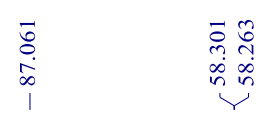

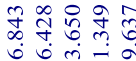

ํำ ำ<smiles>Cc1ccc2sc3c(c2c1)C(=O)N1C2CCCCC2NC31c1ccc(F)cc1</smiles>

4f, ${ }^{13} \mathrm{C}$ NMR $100 \mathrm{MHz}, \mathrm{CDCl}_{3}$

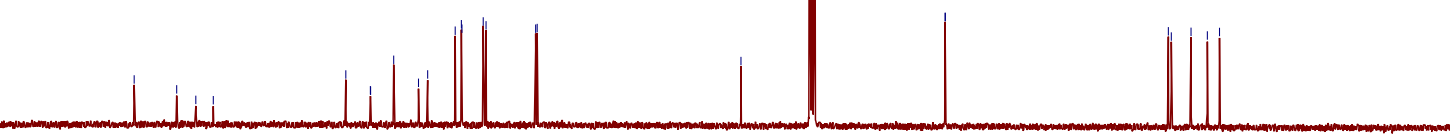

$\begin{array}{lllllllllllllllllllll}190 & 180 & 170 & 160 & 150 & 140 & 130 & 120 & 110 & 100 & \begin{array}{c}90 \\ \mathrm{gpm})\end{array} & 80 & 70 & 60 & 50 & 40 & 30 & 20 & 10 & 0 & -10\end{array}$ 


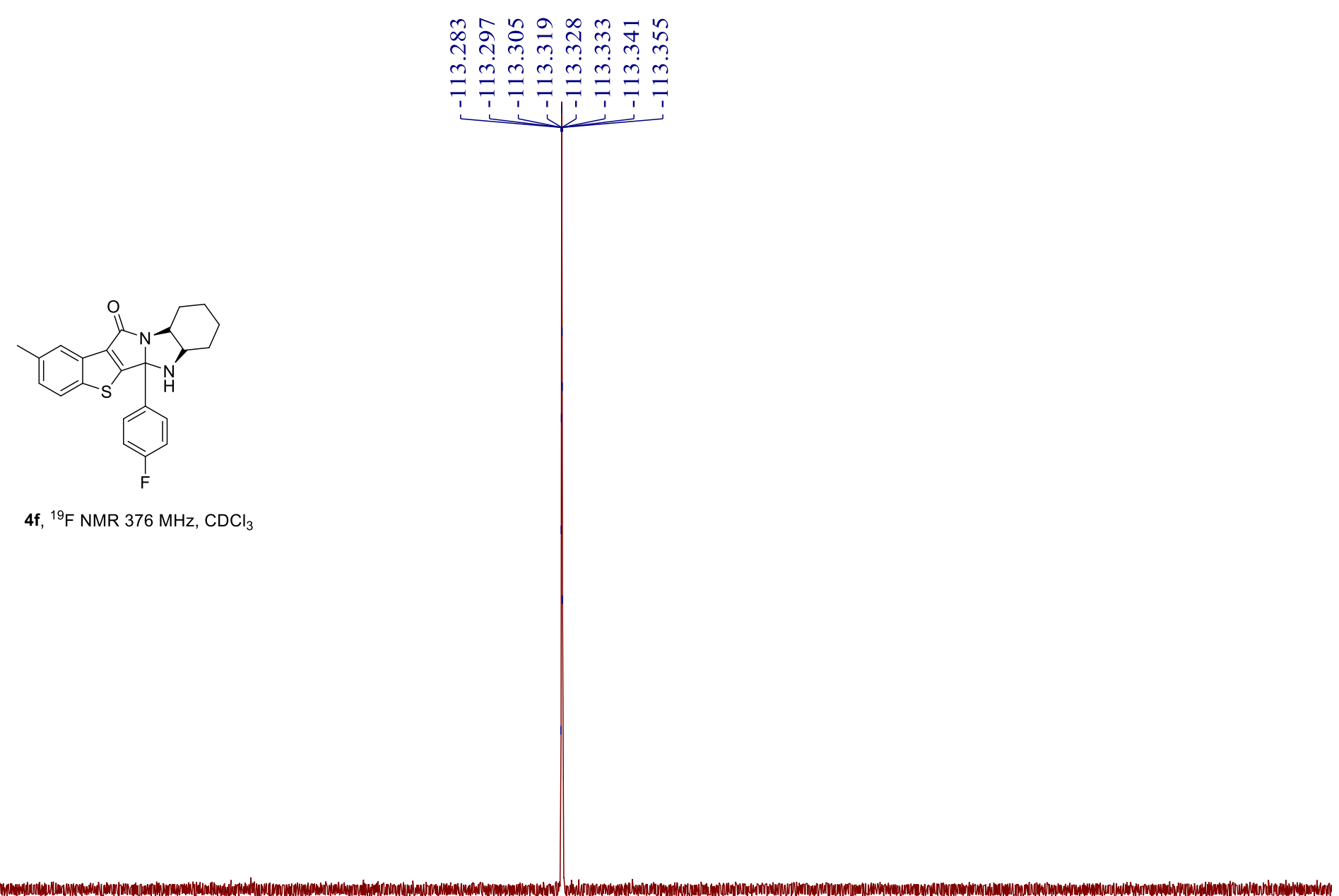

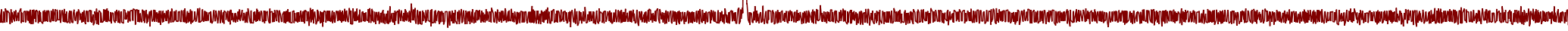

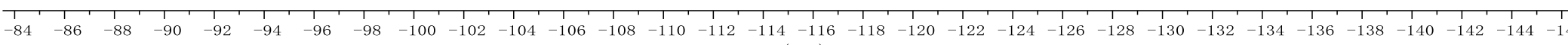




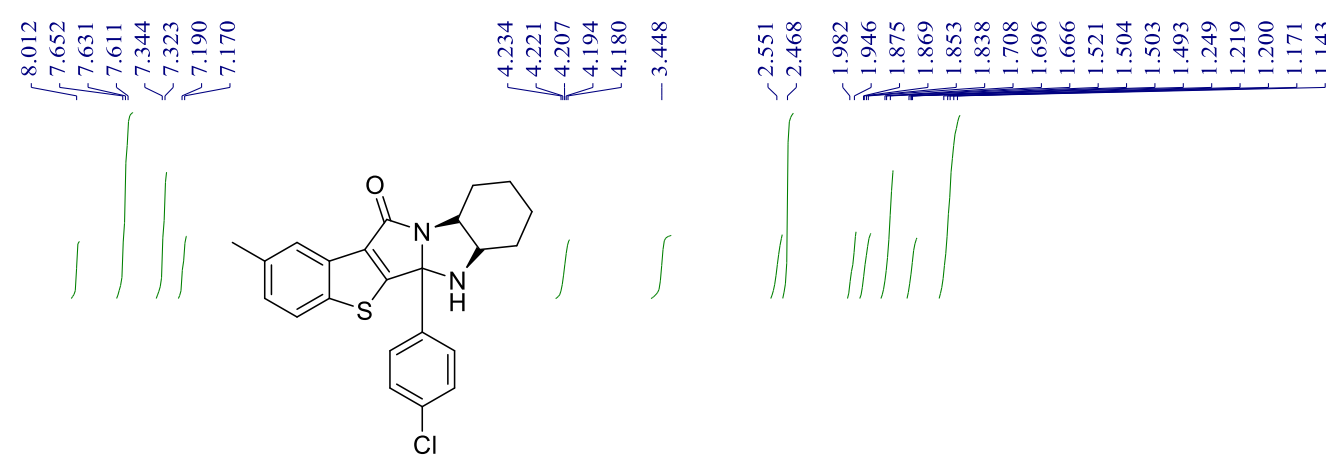

4g, ${ }^{1} \mathrm{H}$ NMR $400 \mathrm{MHz}, \mathrm{CDCl}_{3}$
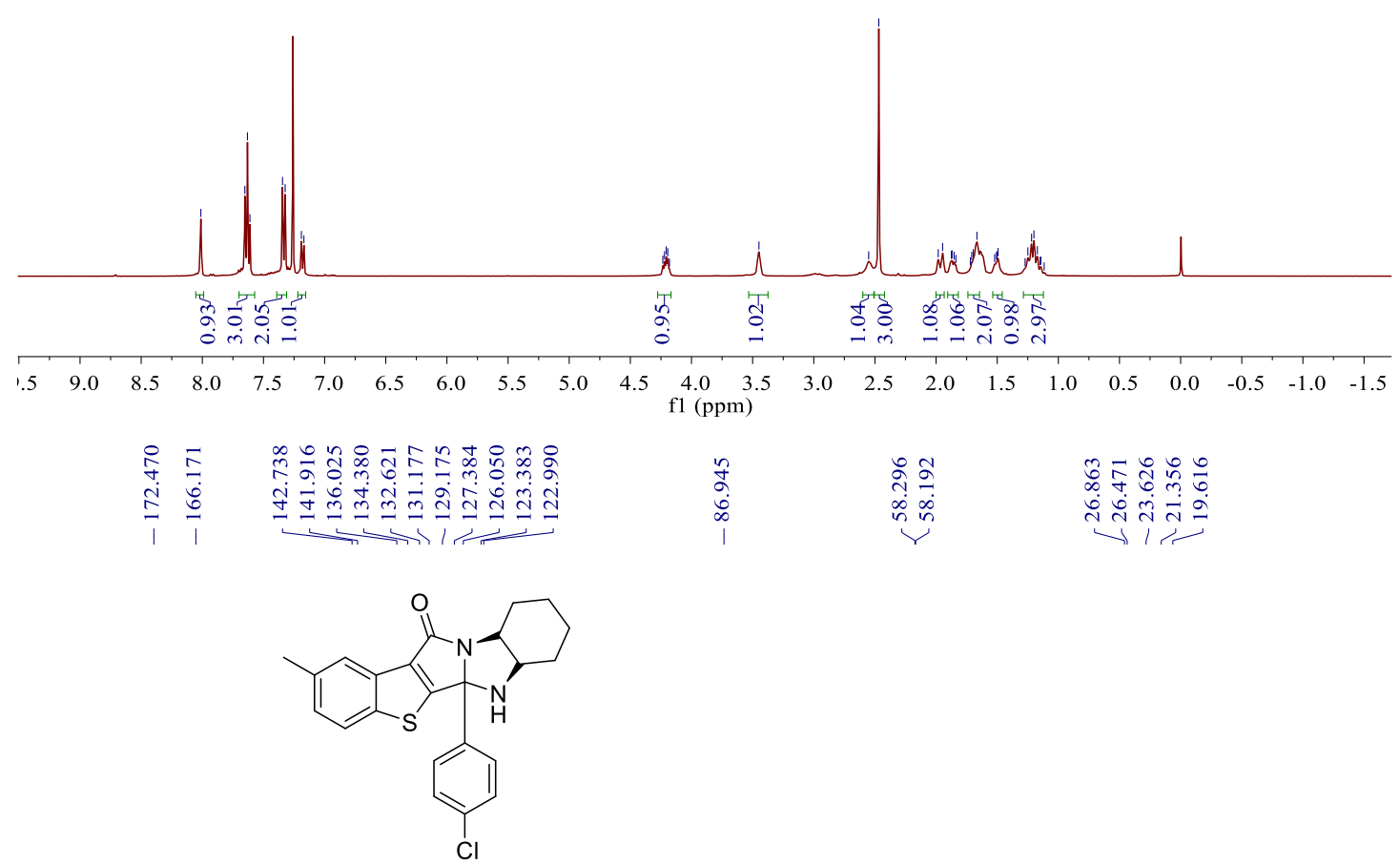

4g, ${ }^{13} \mathrm{C}$ NMR $100 \mathrm{MHz}, \mathrm{CDCl}_{3}$

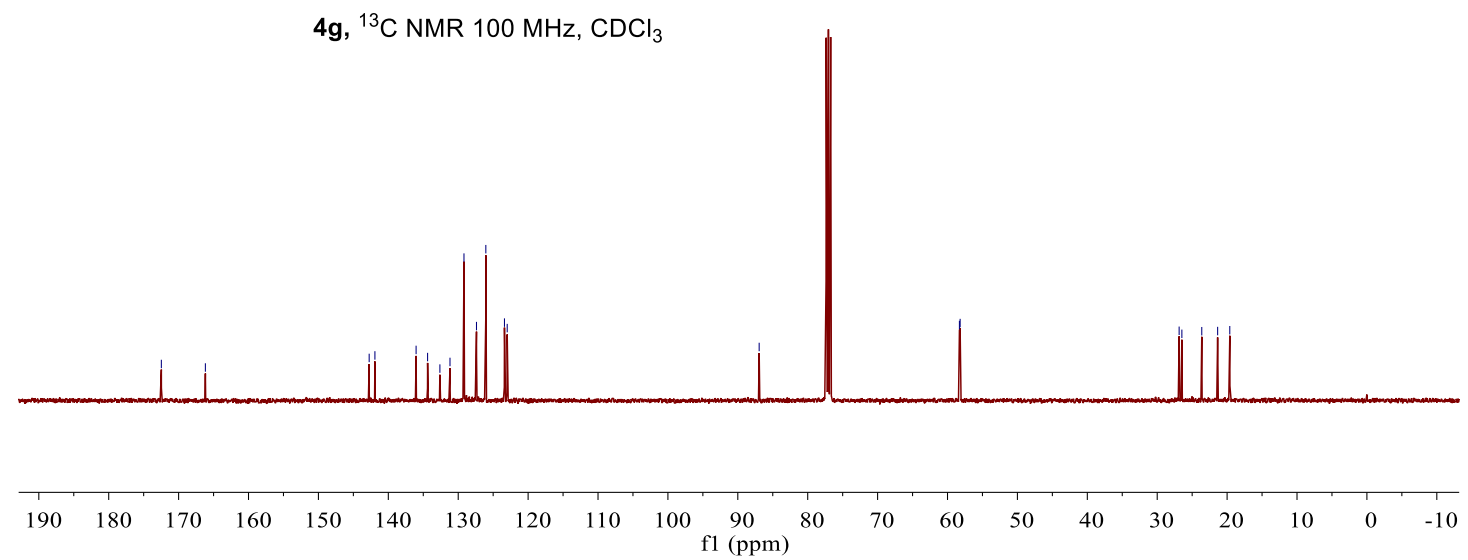




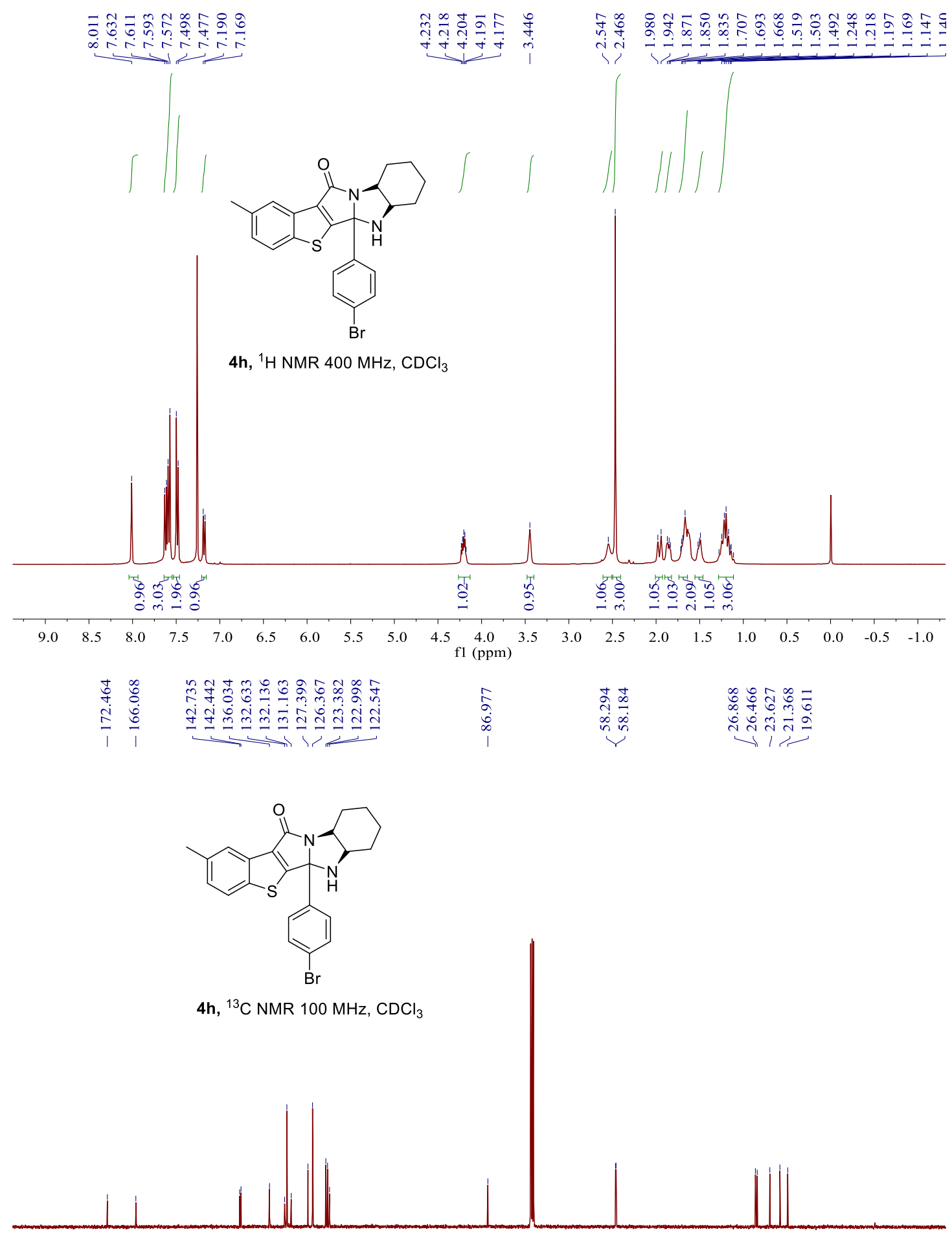

$\begin{array}{lllllllllllllllllllllll}190 & 180 & 170 & 160 & 150 & 140 & 130 & 120 & 110 & 100 & \begin{array}{c}90 \\ \mathrm{f} 1(\mathrm{ppm})\end{array} & 80 & 70 & 60 & 50 & 40 & 30 & 20 & 10 & 0 & -10\end{array}$ 


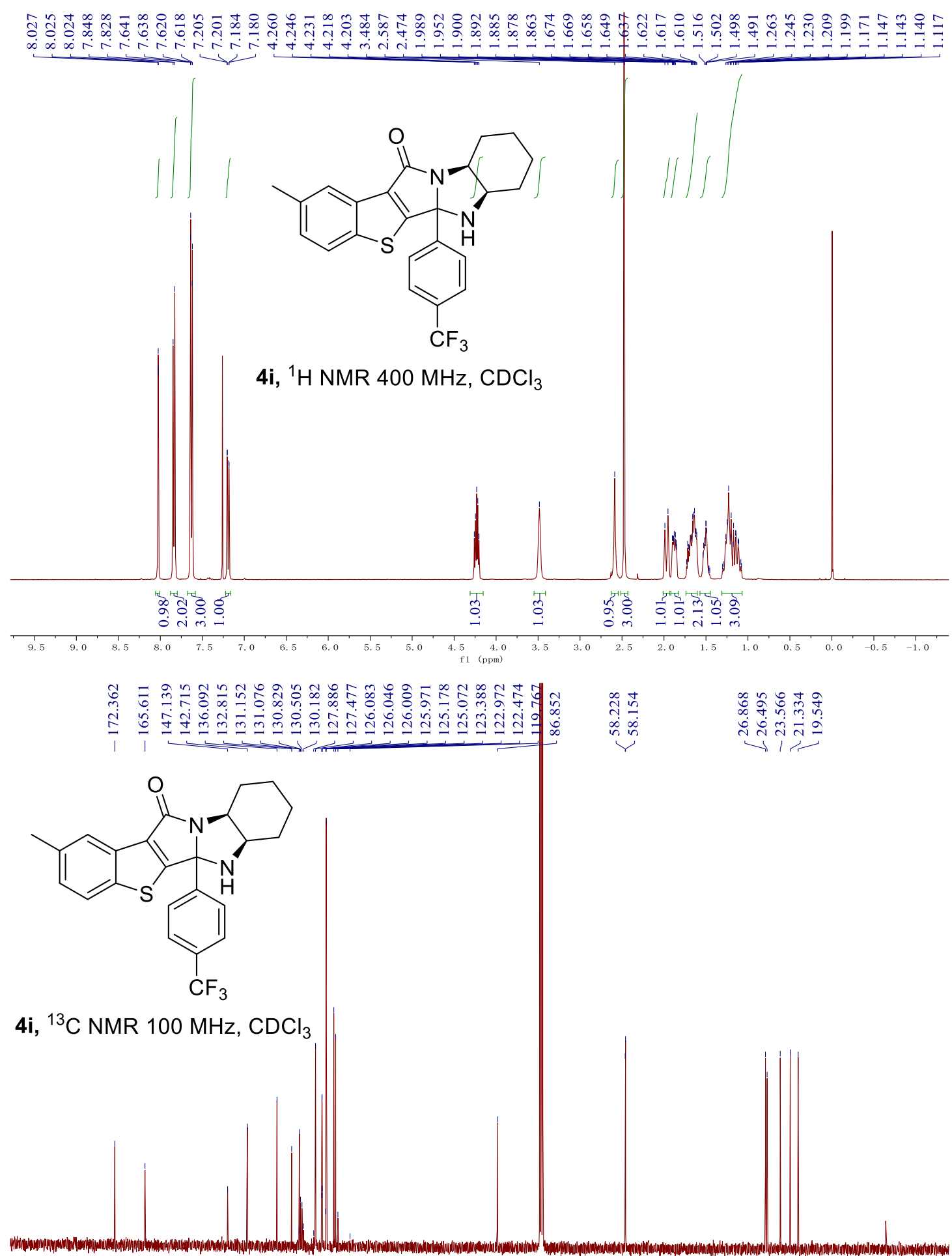

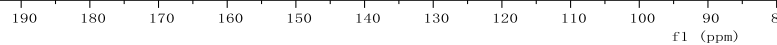




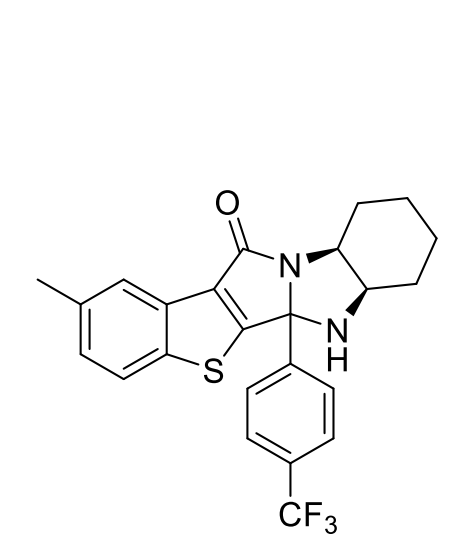

4i, ${ }^{19} \mathrm{~F}$ NMR $376 \mathrm{MHz}, \mathrm{CDCl}_{3}$

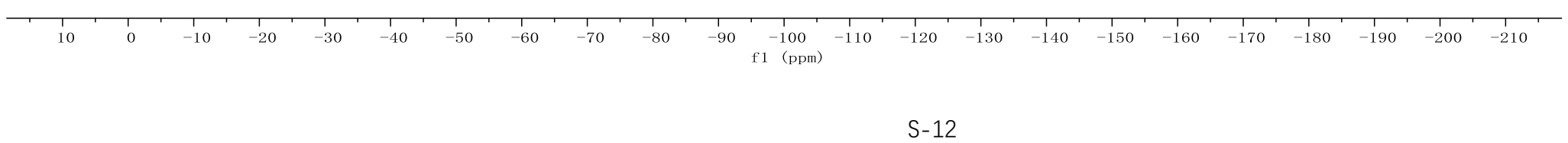




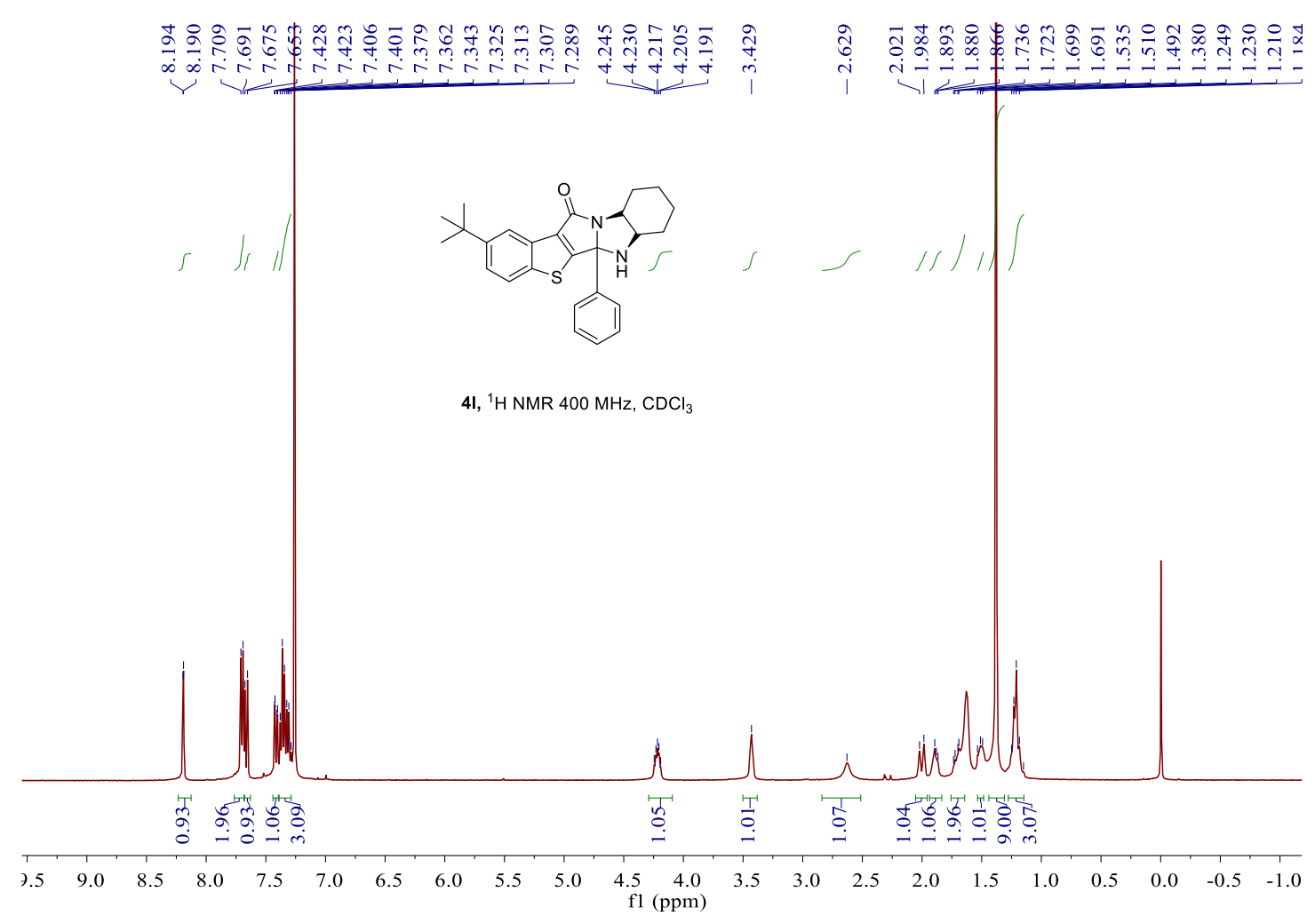

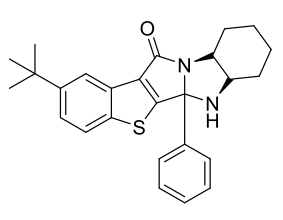

4I, ${ }^{13} \mathrm{C} \mathrm{NMR} 100 \mathrm{MHz}, \mathrm{CDCl}_{3}$

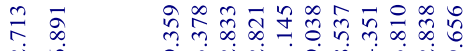

뇸 $\stackrel{8}{\substack{0 \\ \infty \\ 1}}$

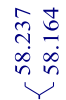

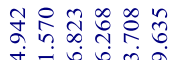

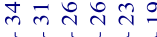

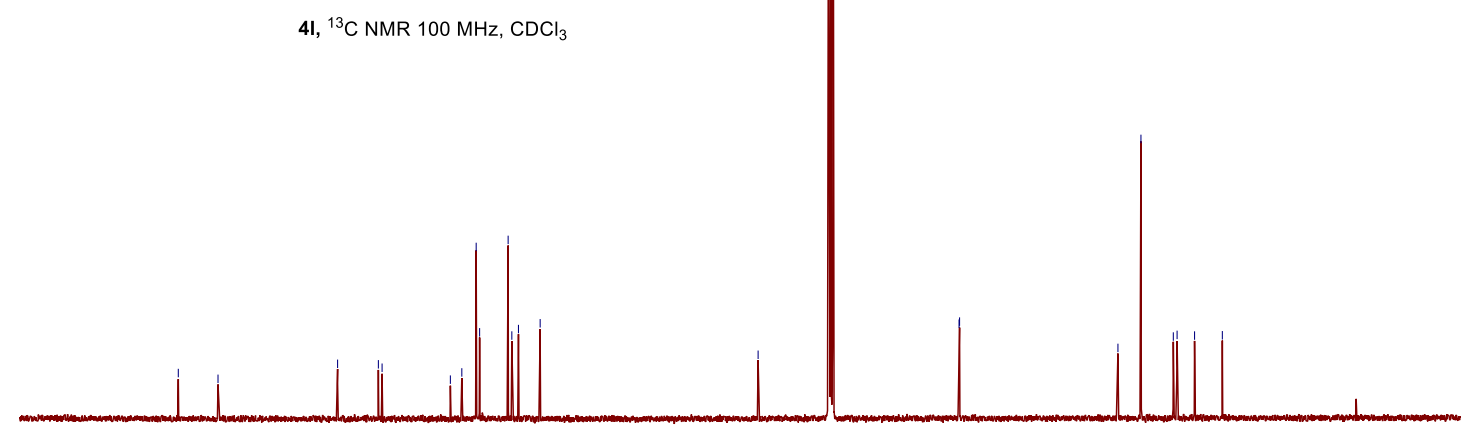

$\begin{array}{llllllllllllllllllll}190 & 180 & 170 & 160 & 150 & 140 & 130 & 120 & 110 & 100 \underset{\mathrm{f} 1}{(\mathrm{ppm})} & 90 & 70 & 60 & 50 & 40 & 30 & 20 & 10 & 0 & -10\end{array}$ 


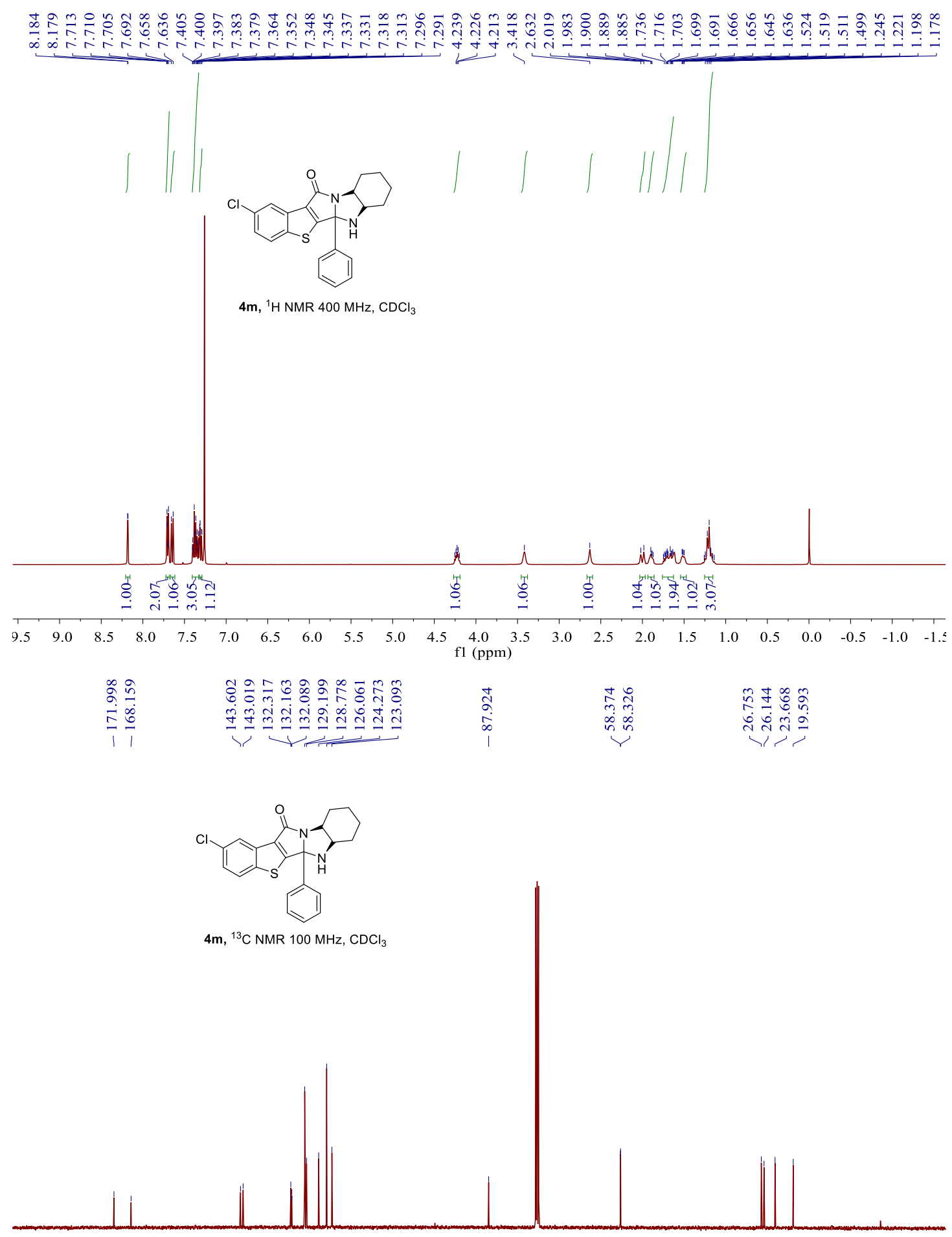

$\begin{array}{llllllllllllllllllll}190 & 180 & 170 & 160 & 150 & 140 & 130 & 120 & 110 & 100 \underset{\mathrm{f} 1}{(\mathrm{ppm})} & 80 & 70 & 60 & 50 & 40 & 30 & 20 & 10 & 0 & -10\end{array}$ 

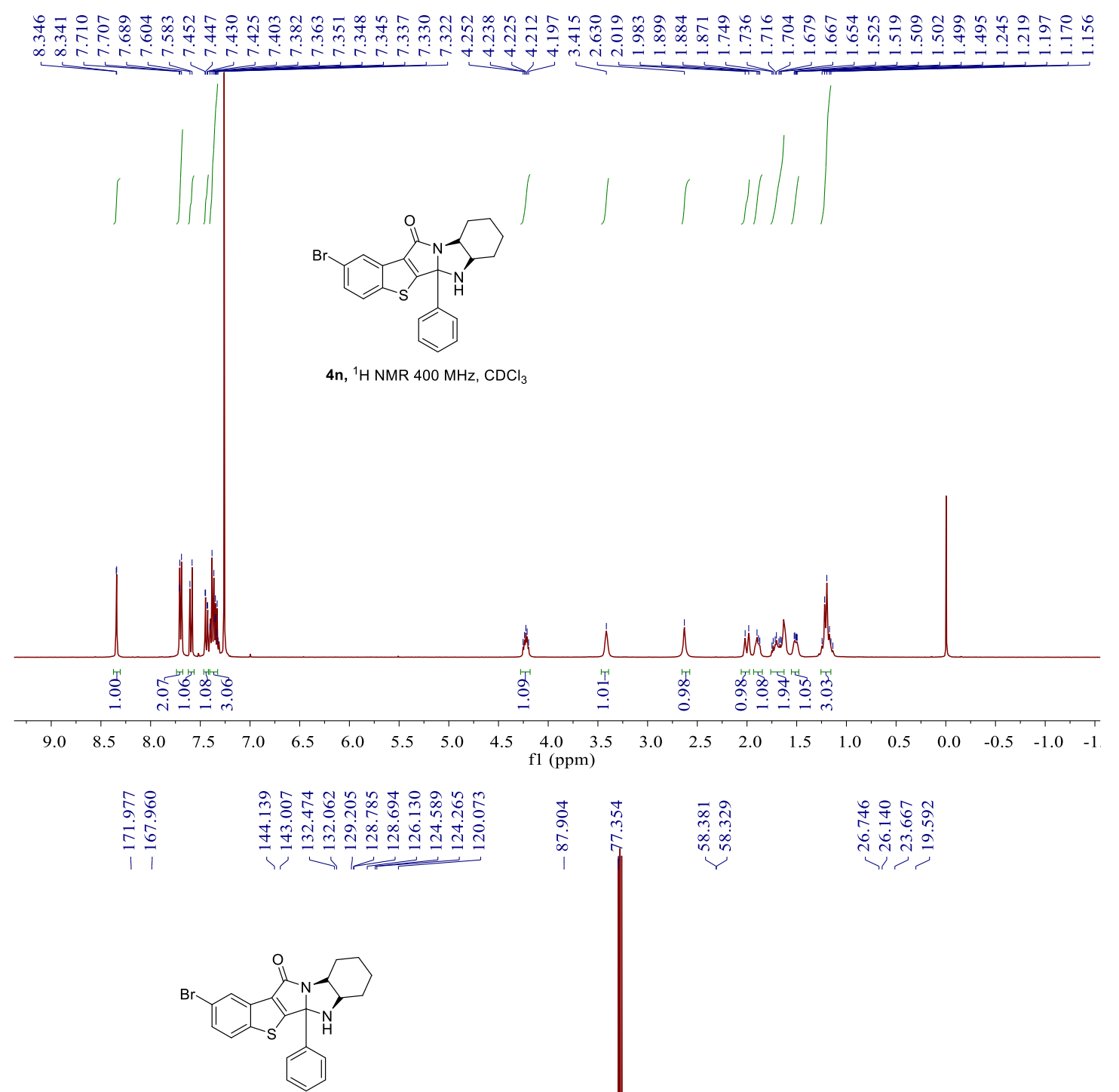

4n, ${ }^{13} \mathrm{C}$ NMR $100 \mathrm{MHz}, \mathrm{CDCl}_{3}$

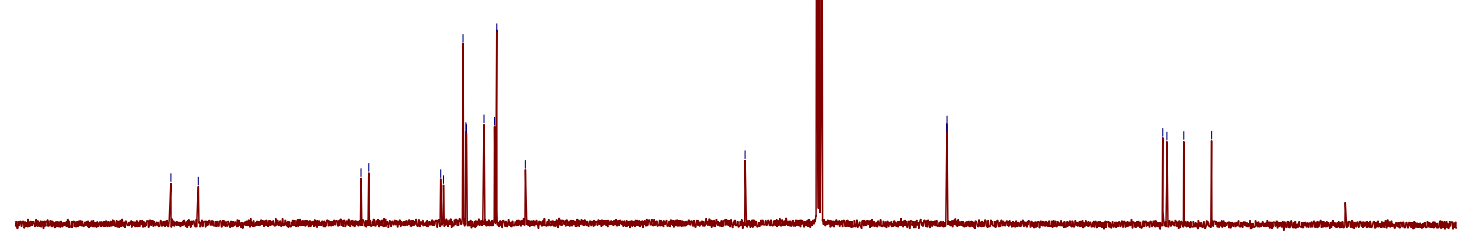

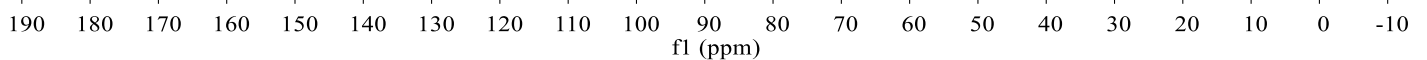




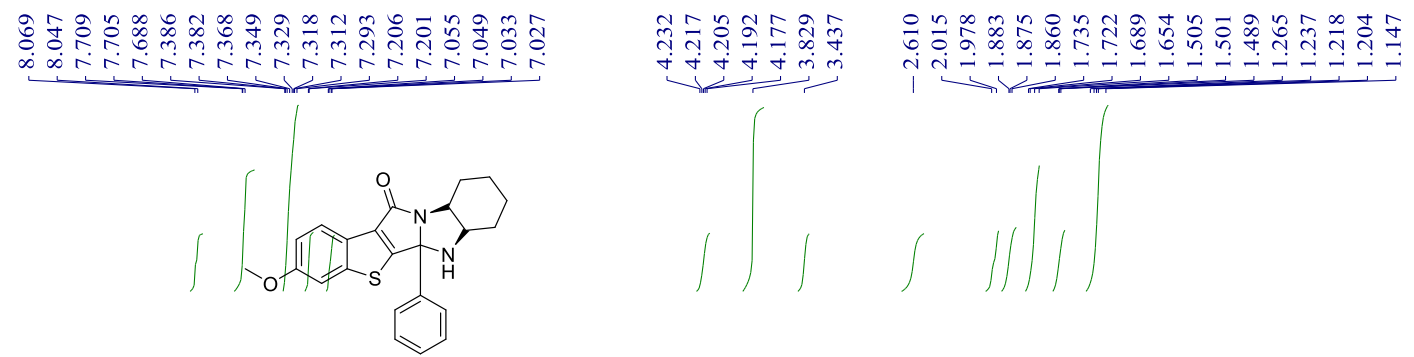

4o, ${ }^{1} \mathrm{H}$ NMR $400 \mathrm{MHz}, \mathrm{CDCl}_{3}$

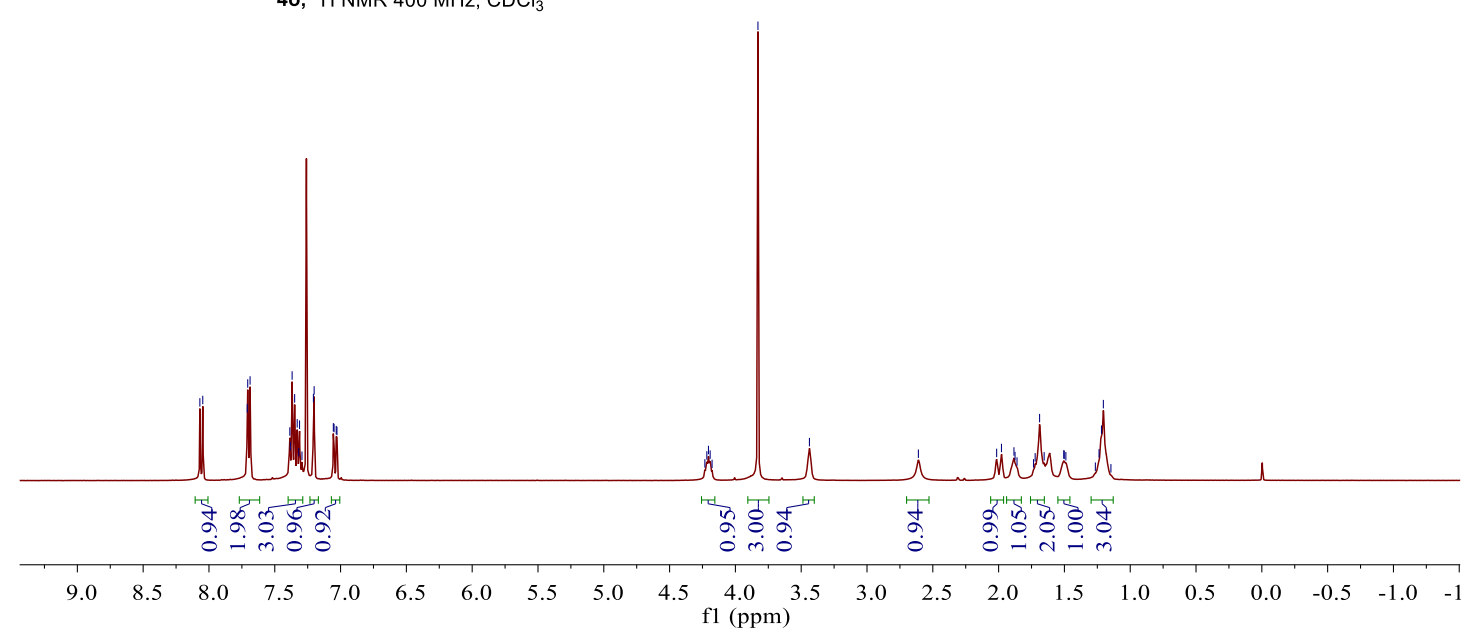

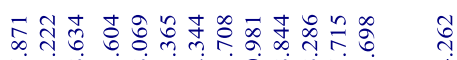

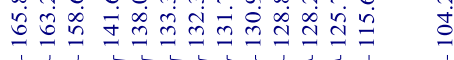

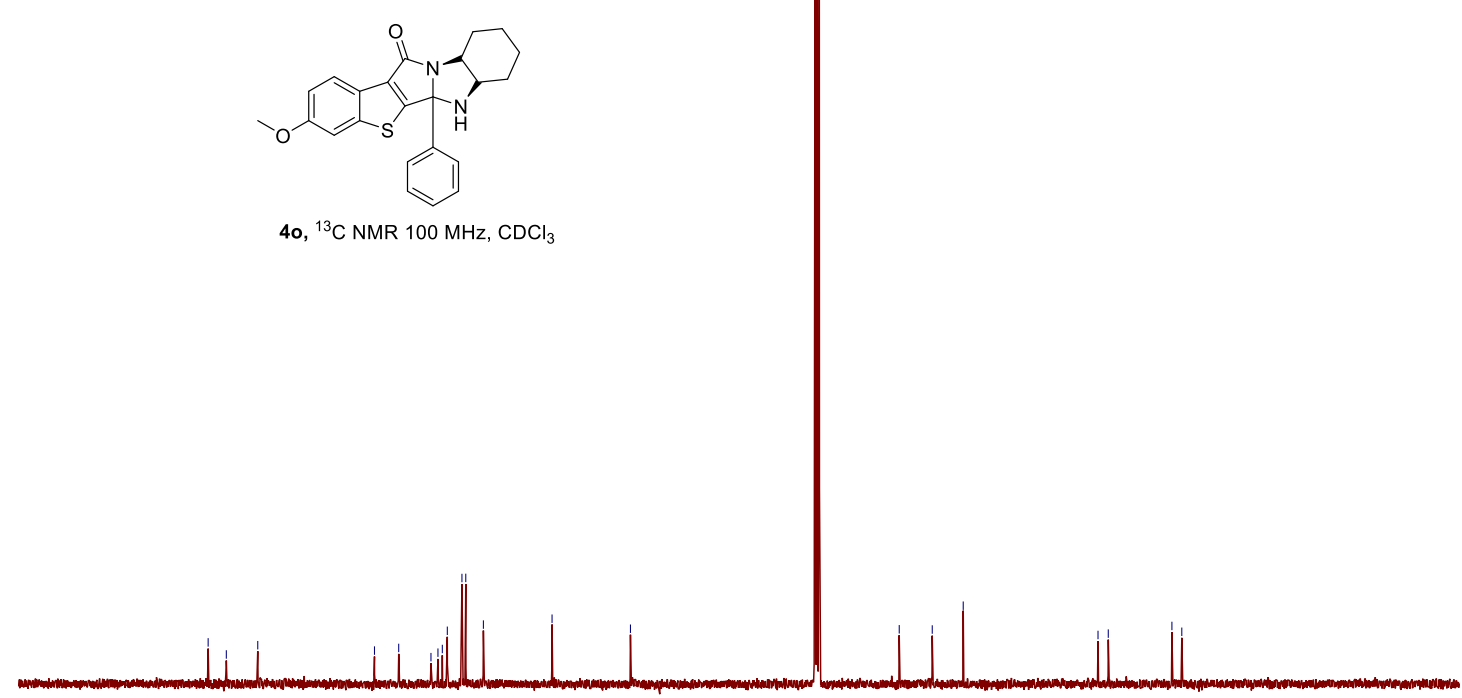

4o, ${ }^{13} \mathrm{C}$ NMR $100 \mathrm{MHz}, \mathrm{CDCl}_{3}$

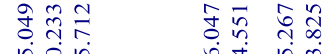

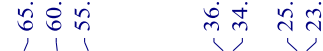

$\begin{array}{llllllllllllllllllll}190 & 180 & 170 & 160 & 150 & 140 & 130 & 120 & 110 & 100 & \begin{array}{c}90 \\ \mathrm{f} 1(\mathrm{ppm})\end{array} & 70 & 60 & 50 & 40 & 30 & 20 & 10 & 0 & -10\end{array}$ 


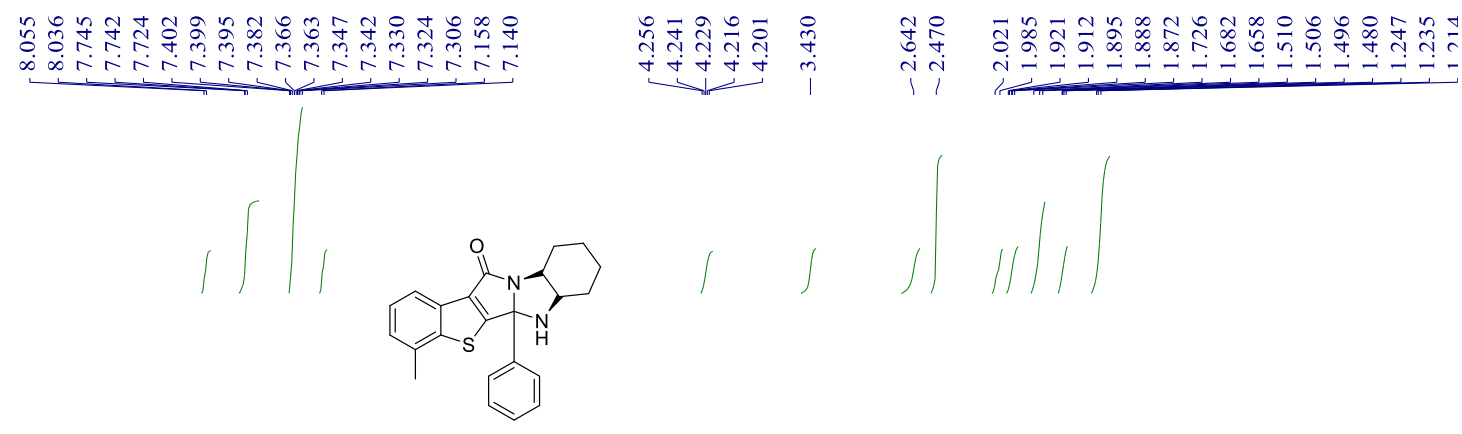

4p, ${ }^{1} \mathrm{H}$ NMR $400 \mathrm{MHz}, \mathrm{CDCl}_{3}$

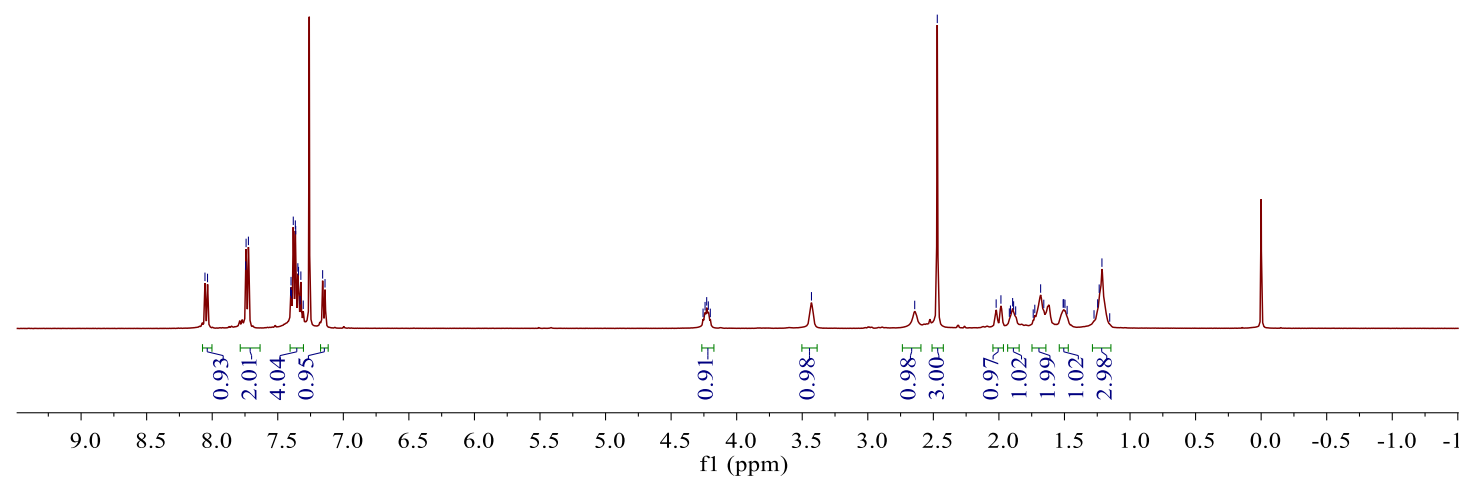

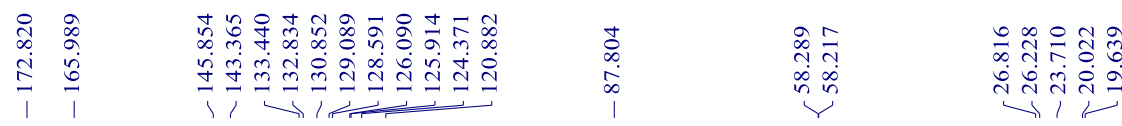

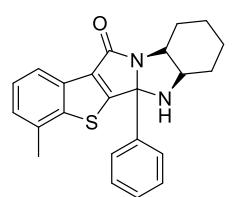

$4 p,{ }^{13} \mathrm{C} \mathrm{NMR} 100 \mathrm{MHz}, \mathrm{CDCl}_{3}$

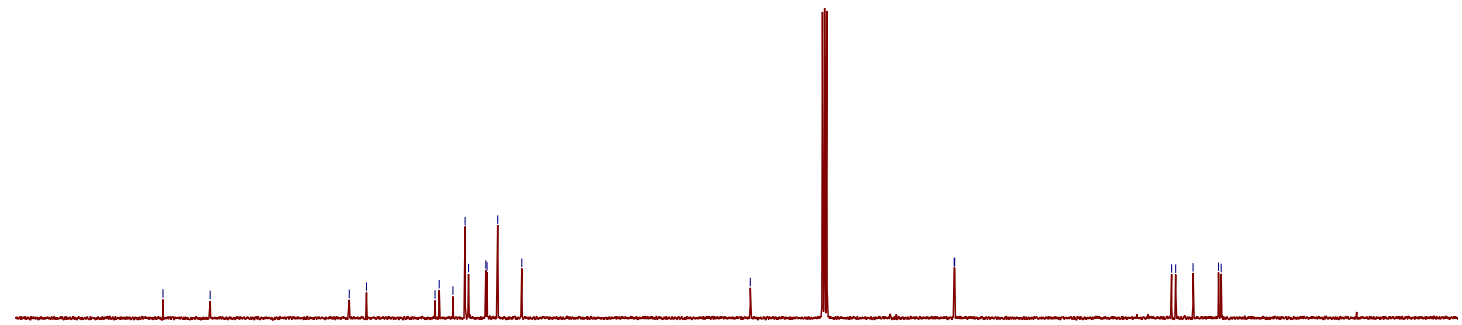

$\begin{array}{lllllllllllllllllllll}190 & 180 & 170 & 160 & 150 & 140 & 130 & 120 & 110 & 100 \underset{\mathrm{f} 1}{\stackrel{9(\mathrm{ppm})}{(\mathrm{ppm})}} & 80 & 70 & 60 & 50 & 40 & 30 & 20 & 10 & 0 & -10\end{array}$ 


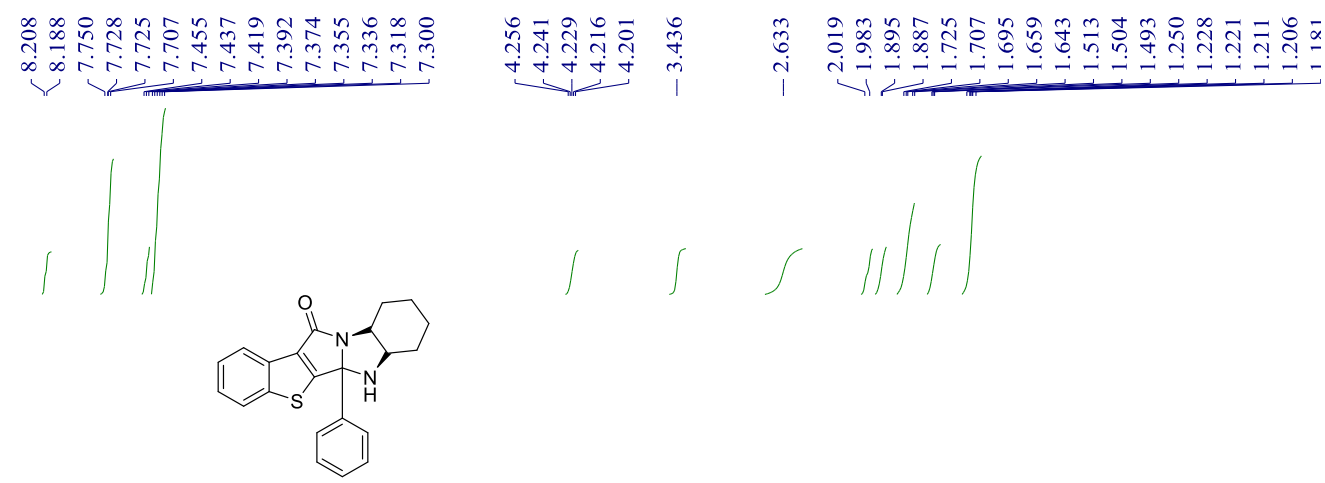

4q, ${ }^{1} \mathrm{H}$ NMR $400 \mathrm{MHz}, \mathrm{CDCl}_{3}$

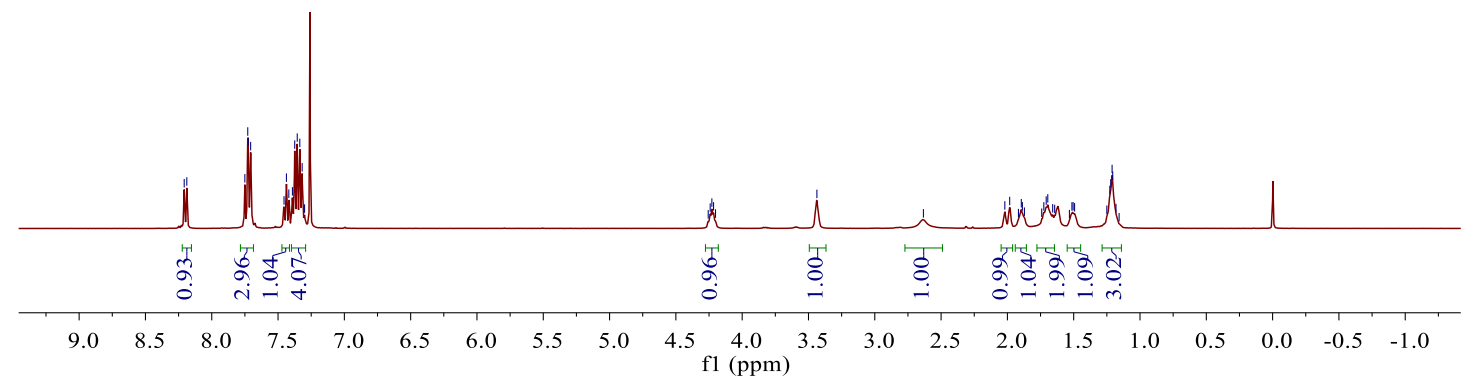

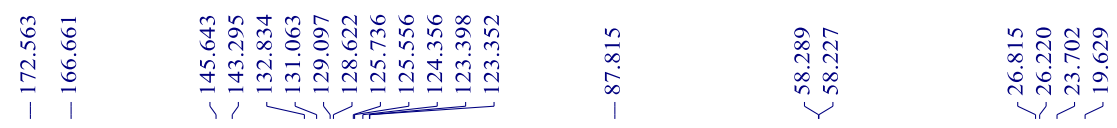

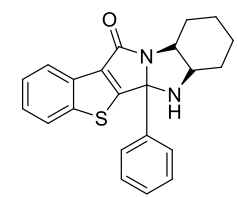

4q, ${ }^{13} \mathrm{C} \mathrm{NMR} 100 \mathrm{MHz}, \mathrm{CDCl}_{3}$

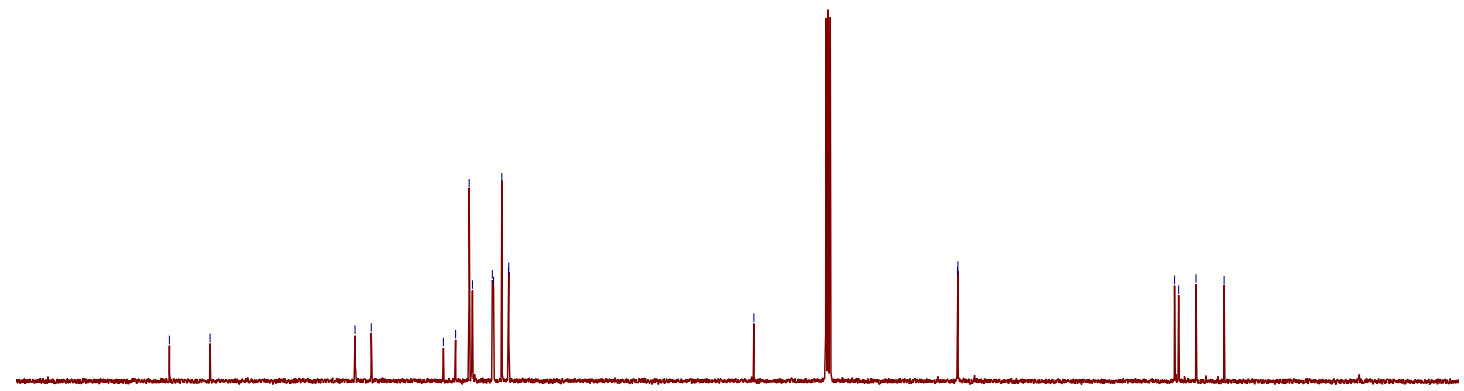

$\begin{array}{llllllllllllllllllll}190 & 180 & 170 & 160 & 150 & 140 & 130 & 120 & 110 & 100 \underset{\mathrm{f} 1}{(\mathrm{ppm})} & 80 & 70 & 60 & 50 & 40 & 30 & 20 & 10 & 0 & -10\end{array}$ 

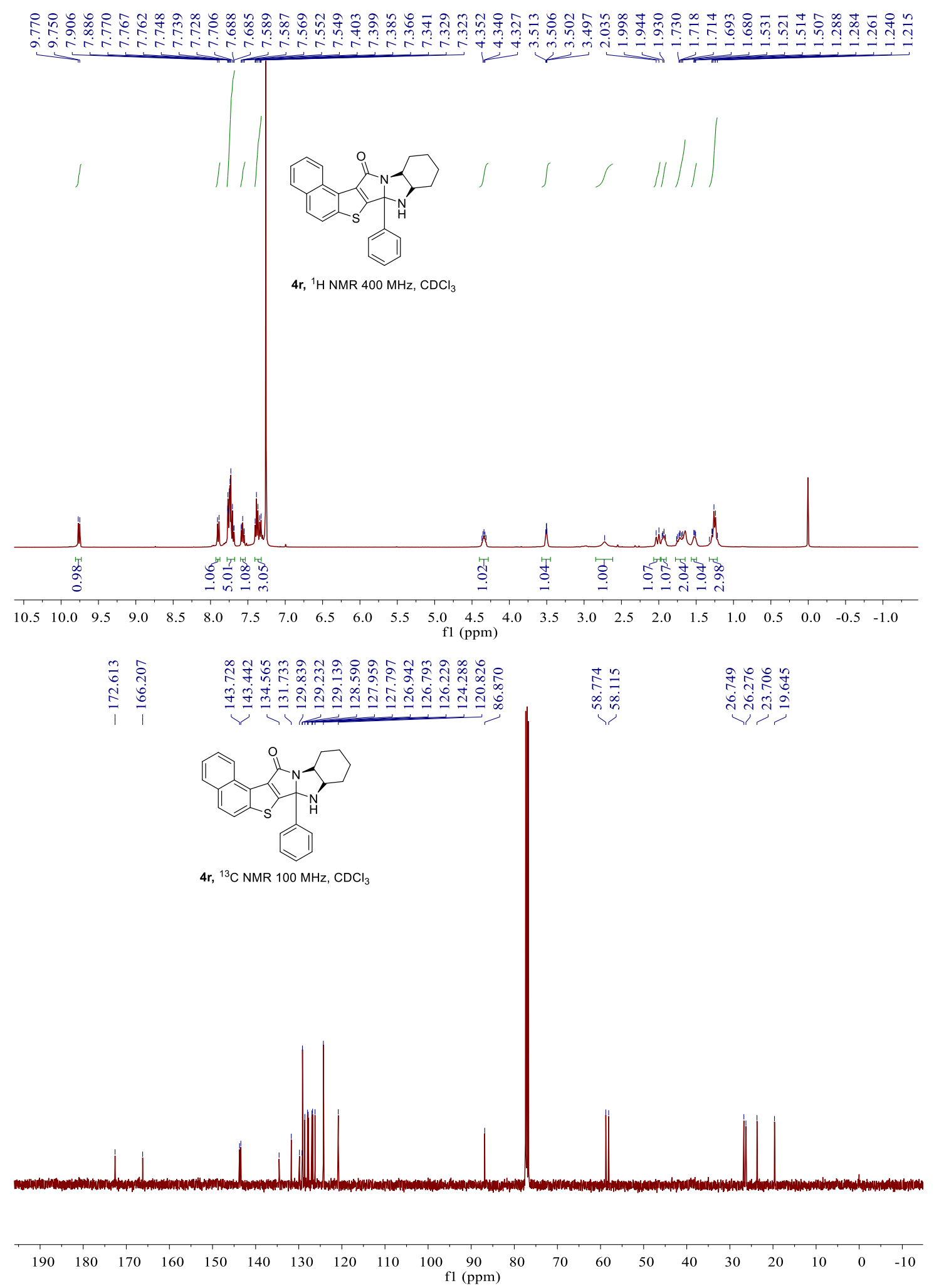

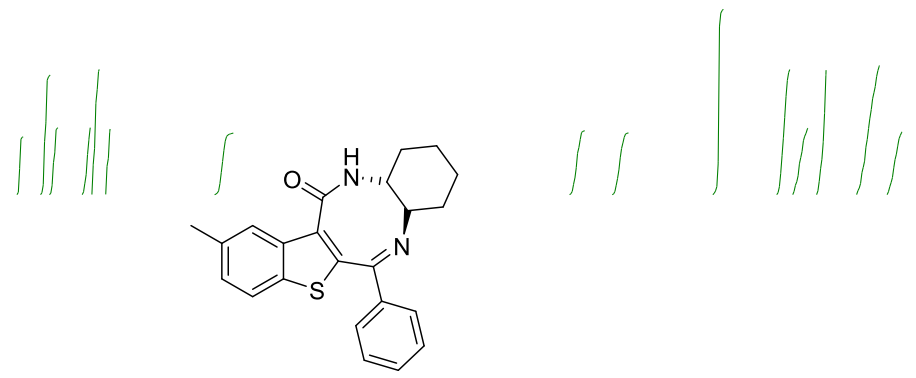

5a, ${ }^{1} \mathrm{H}$ NMR $400 \mathrm{MHz}, \mathrm{CDCl}_{3}$

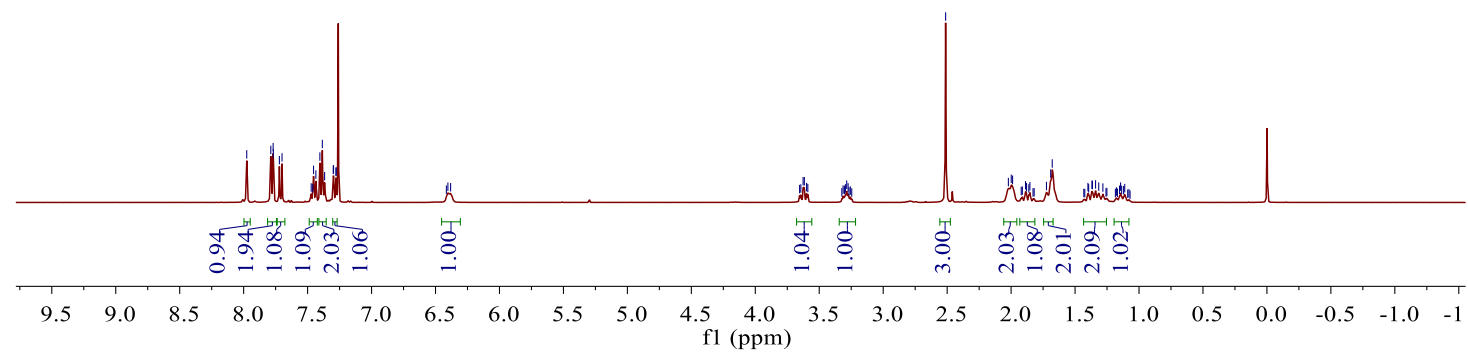

ज̄

药

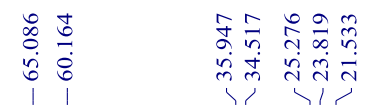

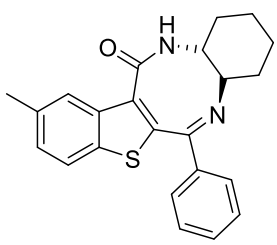

5a, ${ }^{13} \mathrm{C} \mathrm{NMR} 100 \mathrm{MHz}, \mathrm{CDCl}_{3}$

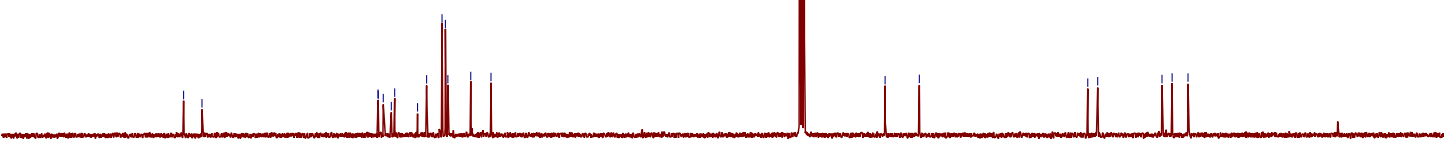

$\begin{array}{llllllllllllllllllllll}190 & 180 & 170 & 160 & 150 & 140 & 130 & 120 & 110 & 100 & 90 & 80 & 70 & 60 & 50 & 40 & 30 & 20 & 10 & 0 & -10\end{array}$ f1 (ppm) 


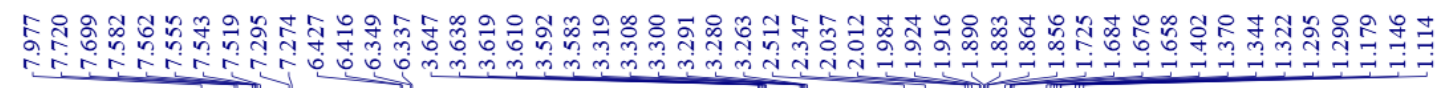

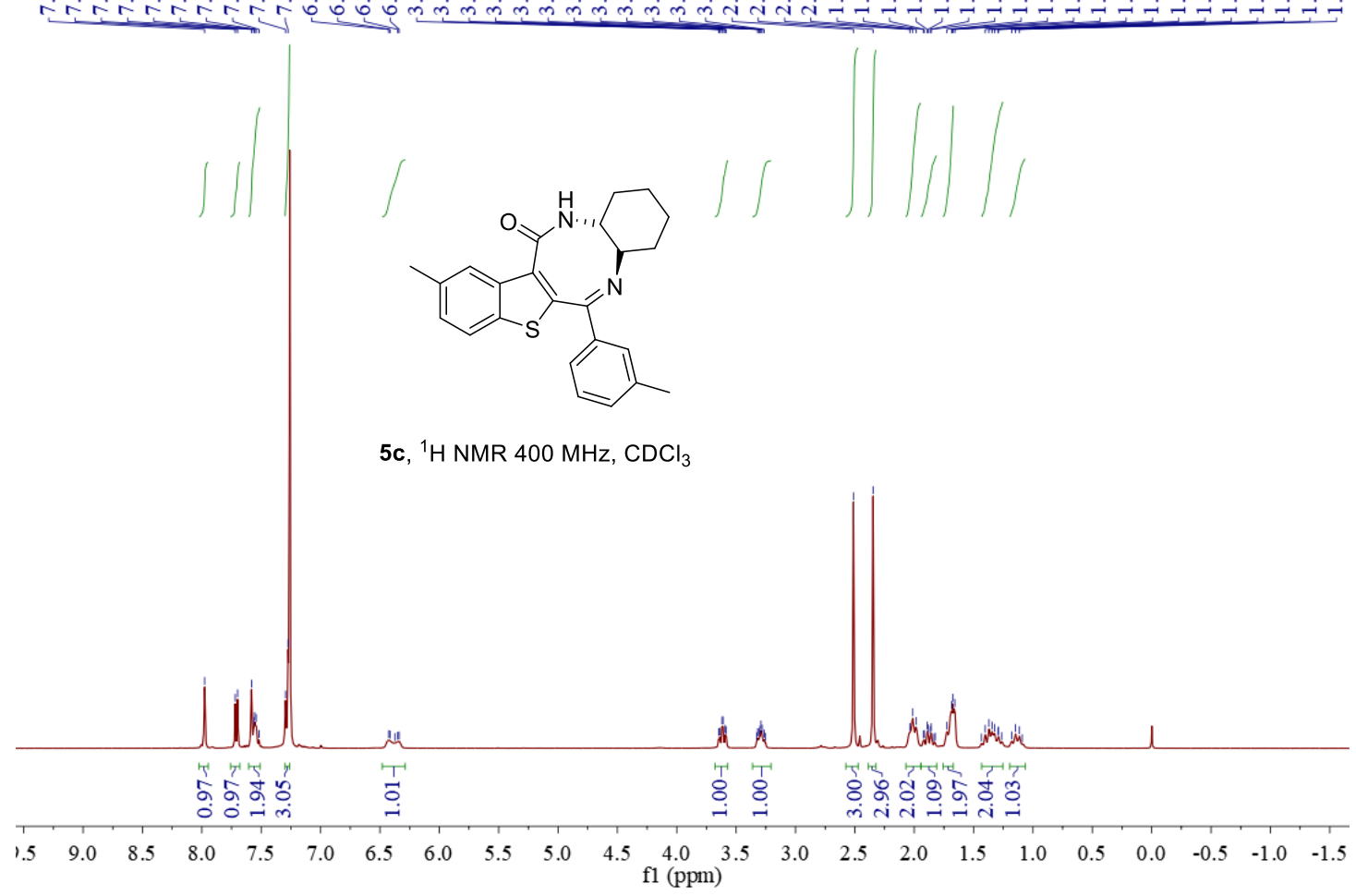

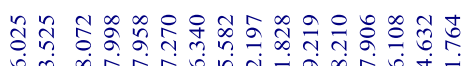

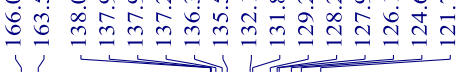

$\begin{array}{ll}\overrightarrow{0} & \infty \\ 0 & \frac{1}{2} \\ 0 & 0\end{array}$

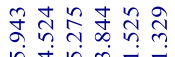

1

ले जेते चे<smiles>Cc1cccc(C2=N[C@@H]3CCCC[C@H]3NC(=O)c3c2sc2ccc(C)cc32)c1</smiles>

5c, ${ }^{13} \mathrm{C} \mathrm{NMR} 100 \mathrm{MHz}, \mathrm{CDCl}_{3}$

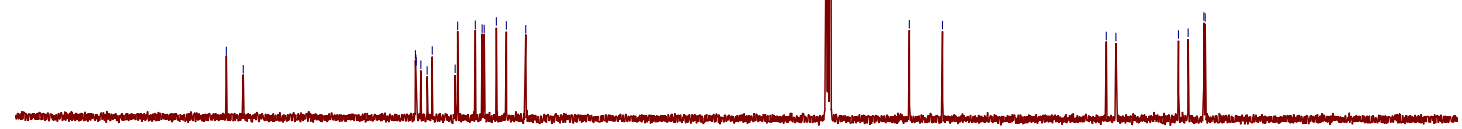

$\begin{array}{lllllllllllllllllllll}190 & 180 & 170 & 160 & 150 & 140 & 130 & 120 & 110 & 100 & \begin{array}{c}90 \\ \mathrm{fl}\end{array} & 80 & 70 & 60 & 50 & 40 & 30 & 20 & 10 & 0 & -10\end{array}$ 


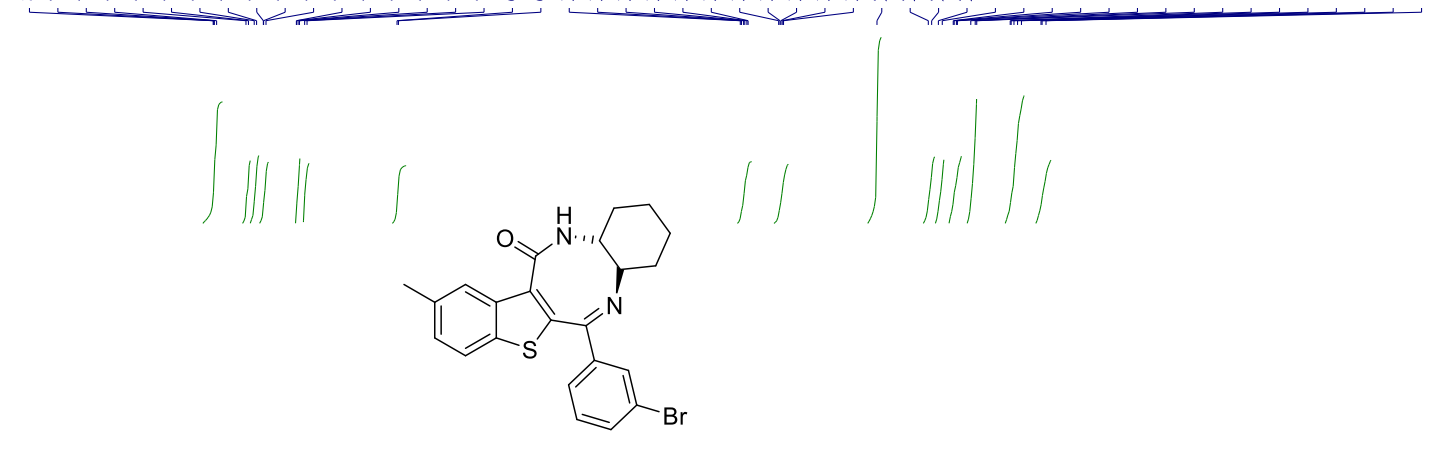

5d, ${ }^{1} \mathrm{H}$ NMR $400 \mathrm{MHz}, \mathrm{CDCl}_{3}$

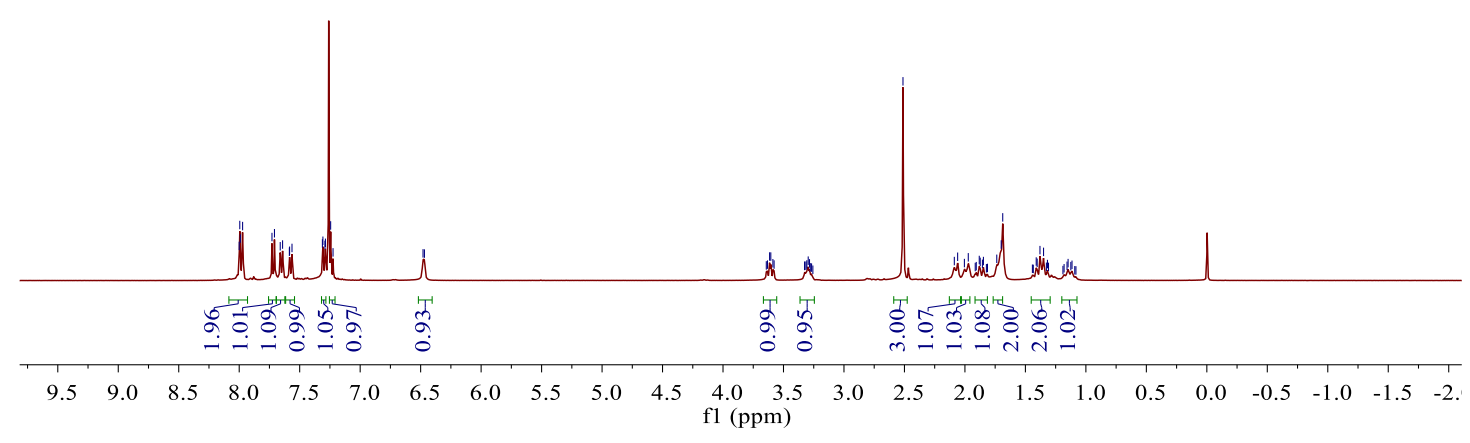

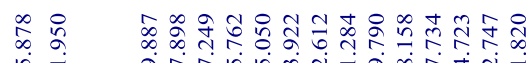

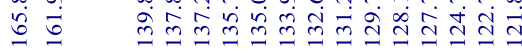
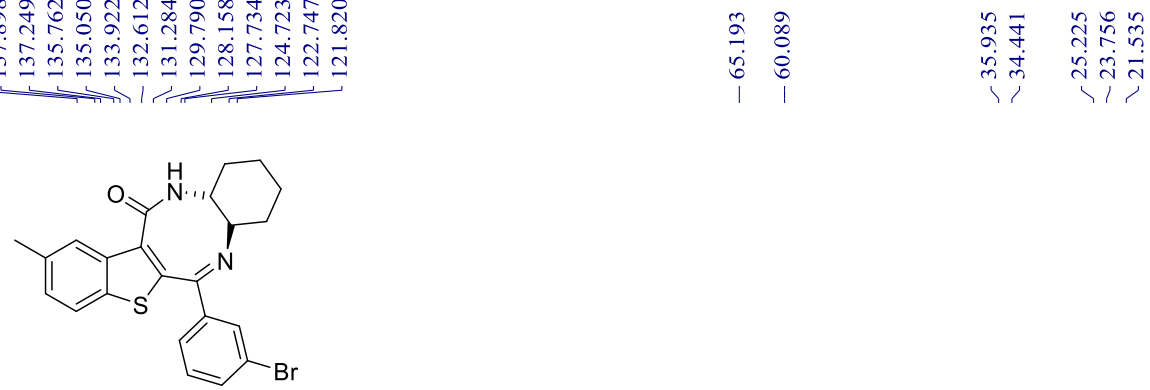

$\mathbf{5 d},{ }^{13} \mathrm{C}$ NMR $100 \mathrm{MHz}, \mathrm{CDCl}_{3}$

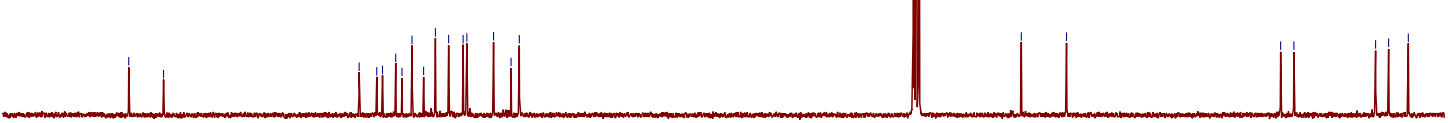

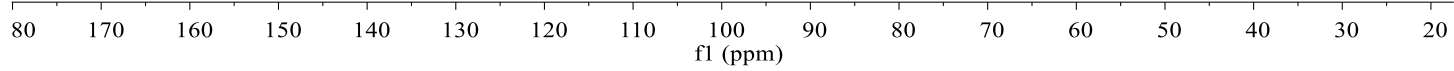




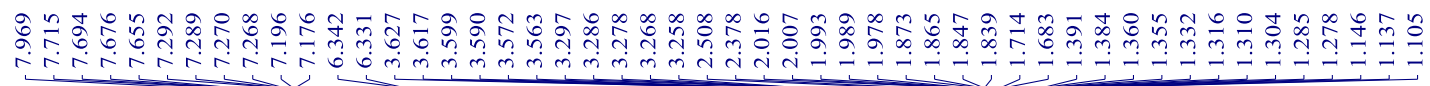

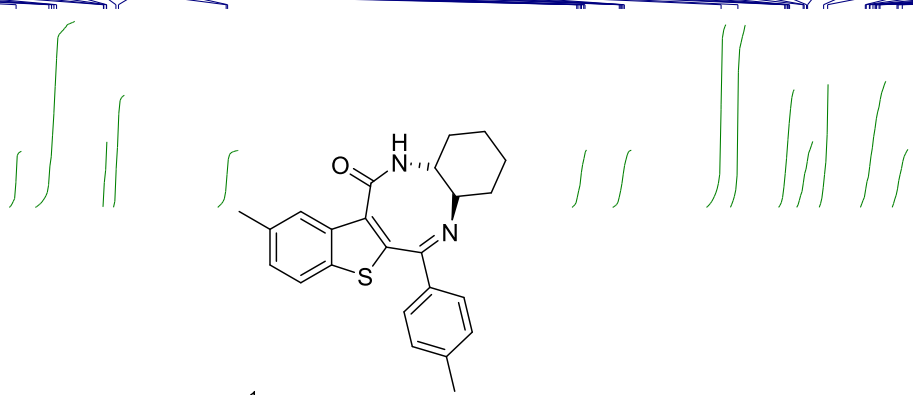

5e, ${ }^{1} \mathrm{H}$ NMR $400 \mathrm{MHz}, \mathrm{CDCl}_{3}$

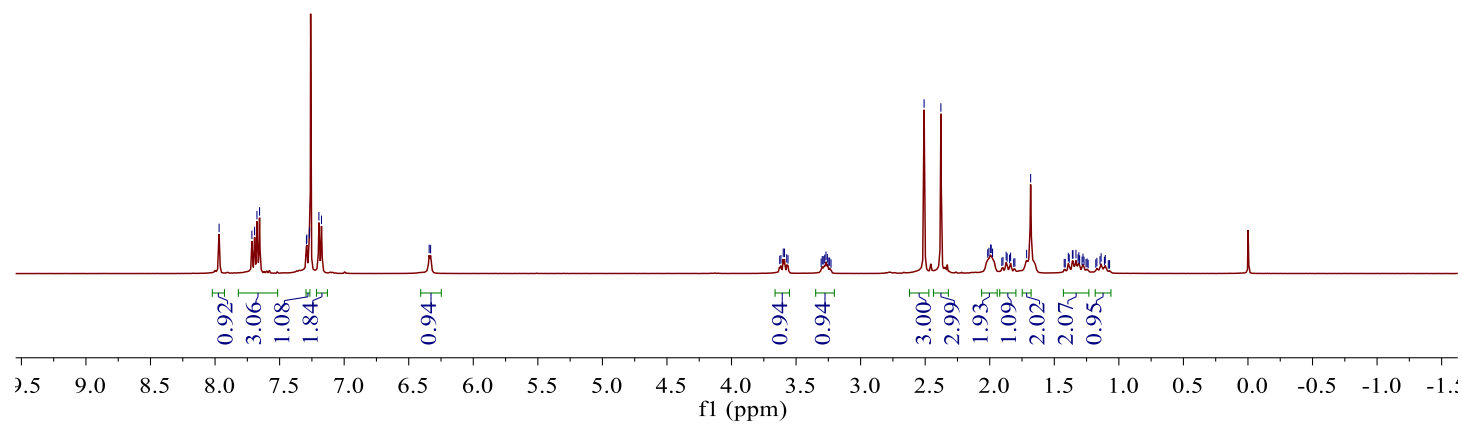

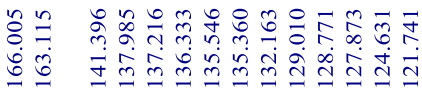

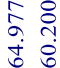

กิ๊

ले प्ते ते

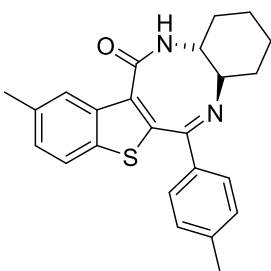

5e, ${ }^{13} \mathrm{C}$ NMR $100 \mathrm{MHz}, \mathrm{CDCl}_{3}$

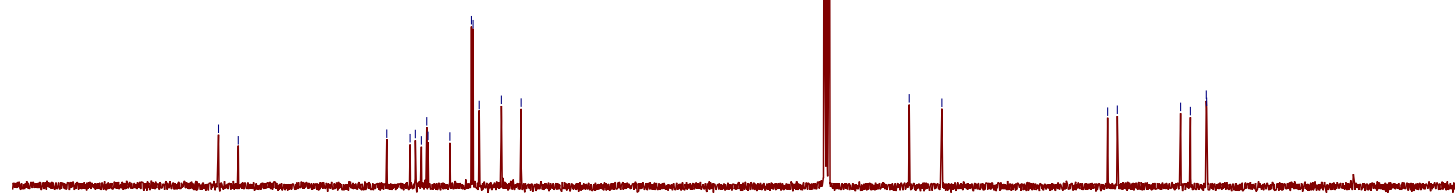

$\begin{array}{llllllllllllllllllll}190 & 180 & 170 & 160 & 150 & 140 & 130 & 120 & 110 & 100 \underset{\mathrm{f} 1}{(\mathrm{ppm})} \mathbf{9 0} & 80 & 70 & 60 & 50 & 40 & 30 & 20 & 10 & 0 & -10\end{array}$ 


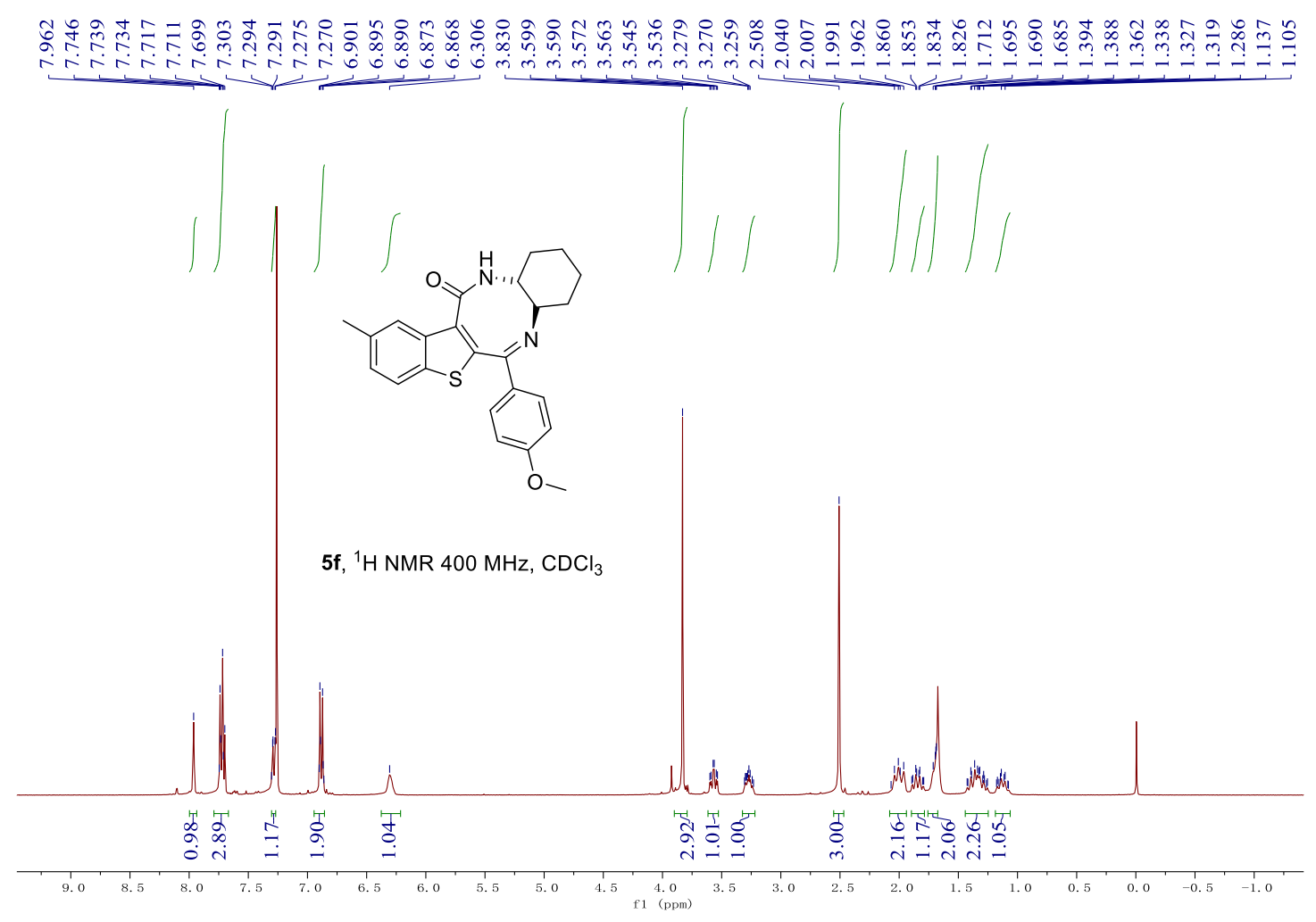

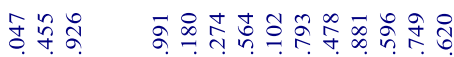

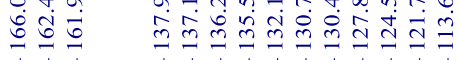

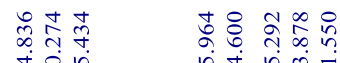

它总㶽

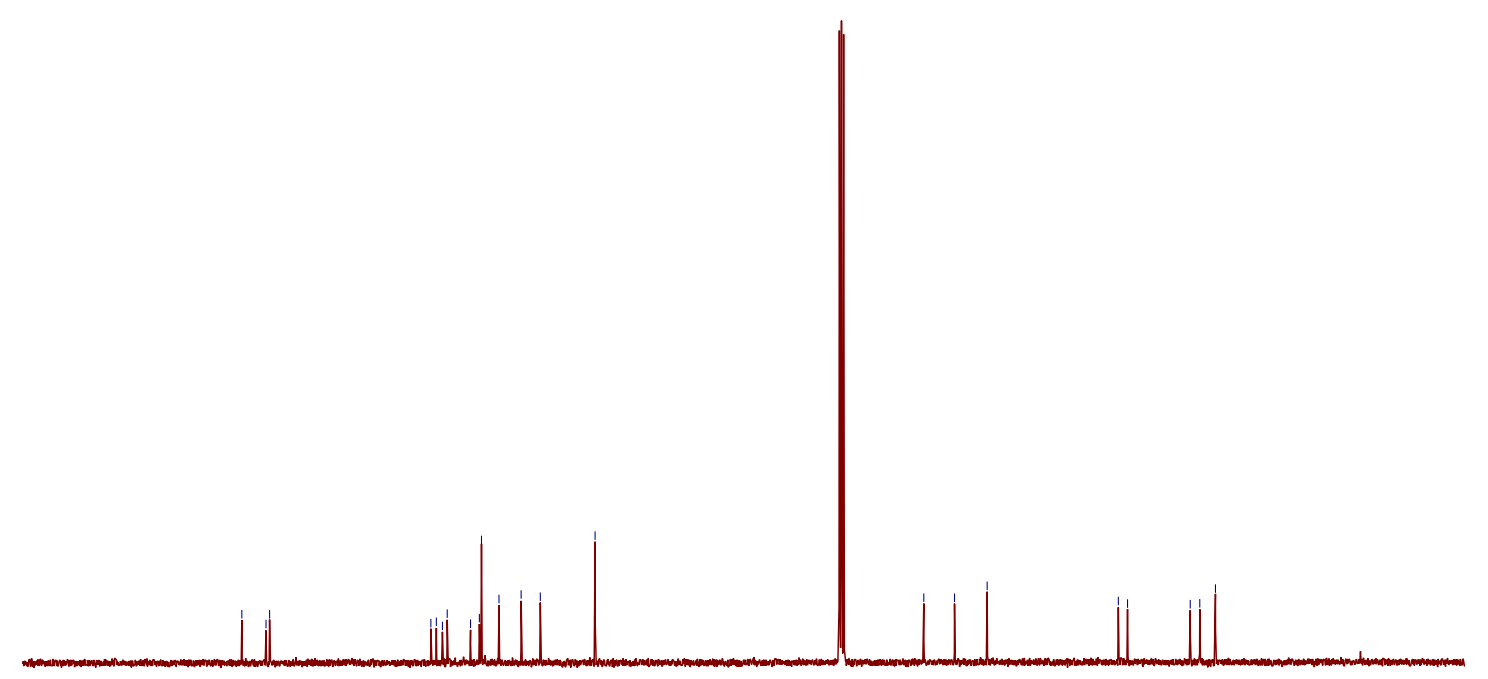

$\begin{array}{lllllllllllllllllllll}190 & 180 & 170 & 160 & 150 & 140 & 130 & 120 & 110 & 100 & \underset{\mathrm{f} 1(\mathrm{ppm})}{90} & 80 & 70 & 60 & 50 & 40 & 30 & 20 & 10 & 0 & -10\end{array}$

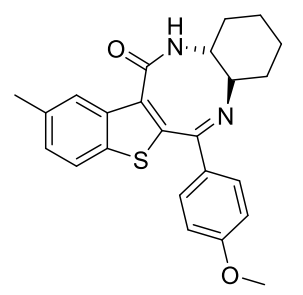

5f, ${ }^{13} \mathrm{C} \mathrm{NMR} 100 \mathrm{MHz}, \mathrm{CDCl}_{3}$ 


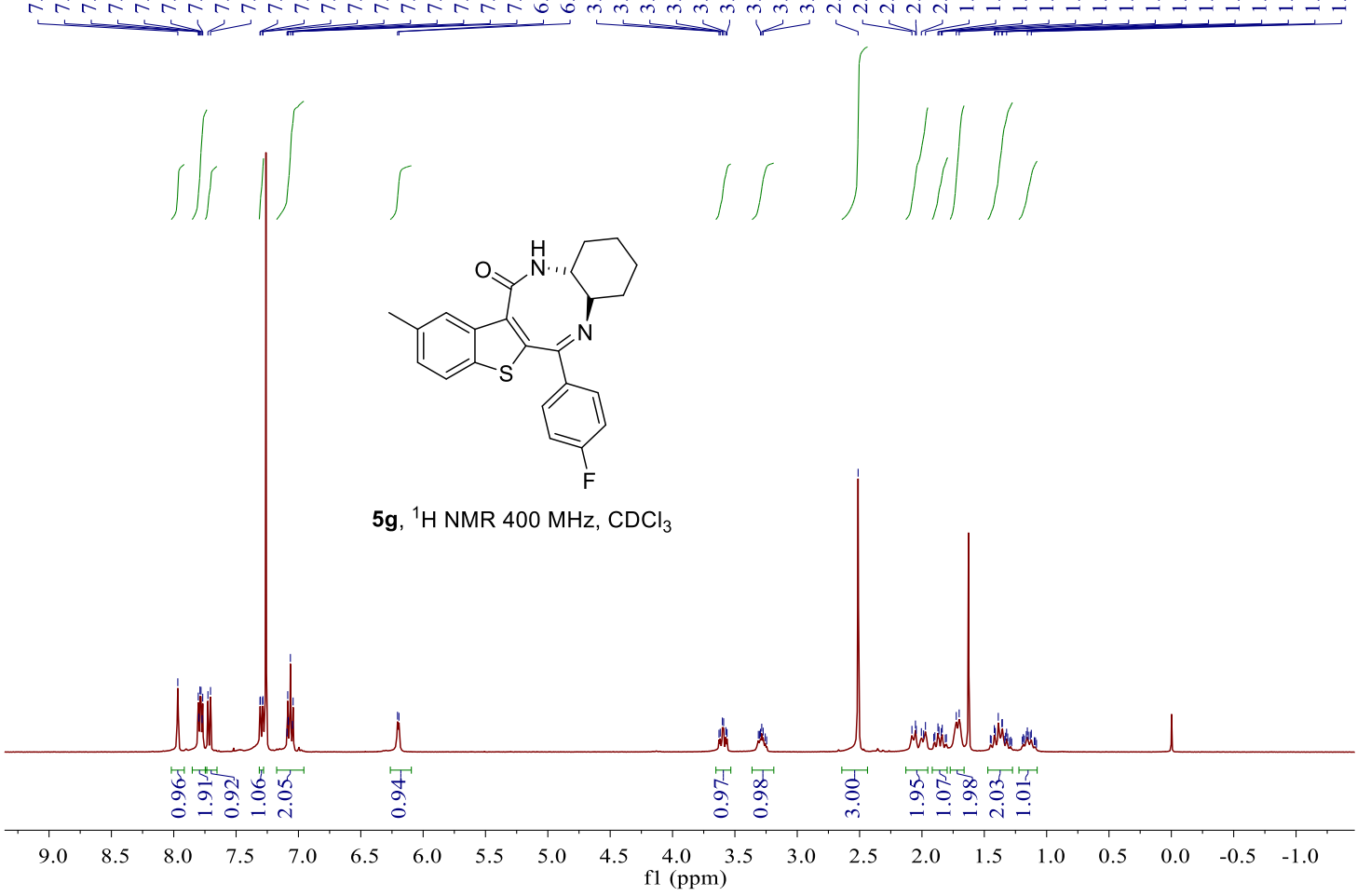

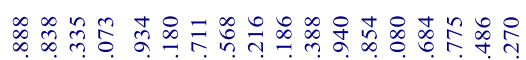

vi்

$\begin{array}{ll}n & + \\ 0 & \infty \\ 0 & 0 \\ 0 & 0 \\ 1 & 1\end{array}$

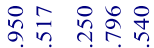

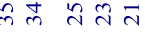

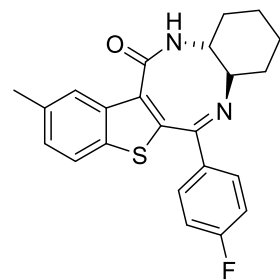

$\mathbf{5 g},{ }^{13} \mathrm{C} \mathrm{NMR} 100 \mathrm{MHz}, \mathrm{CDCl}_{3}$

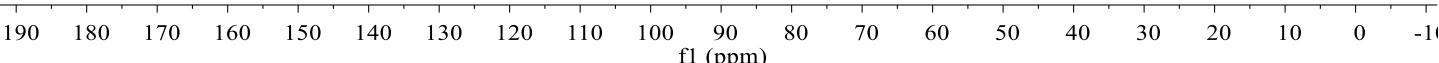



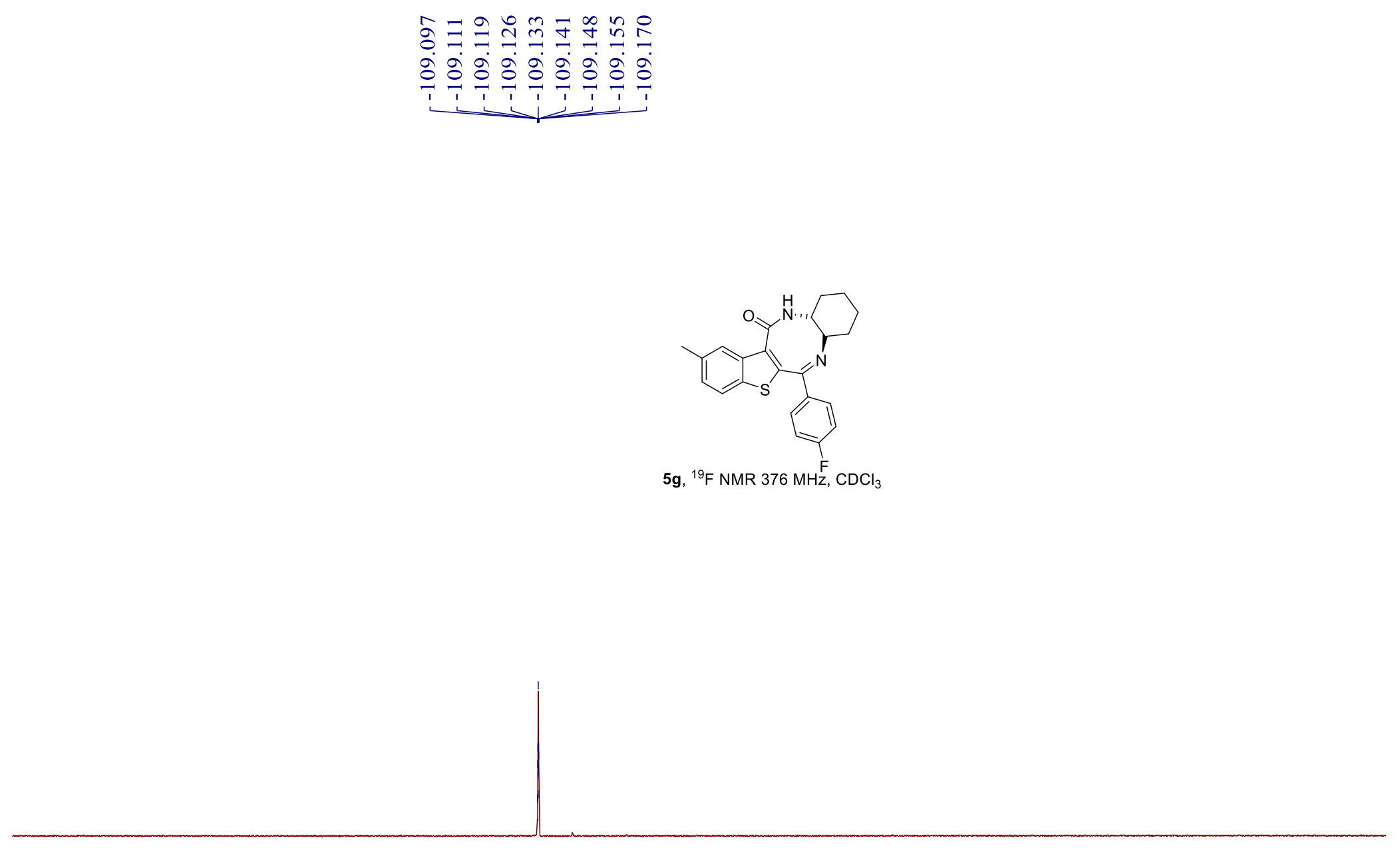

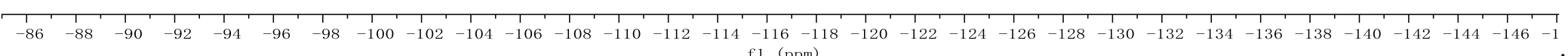




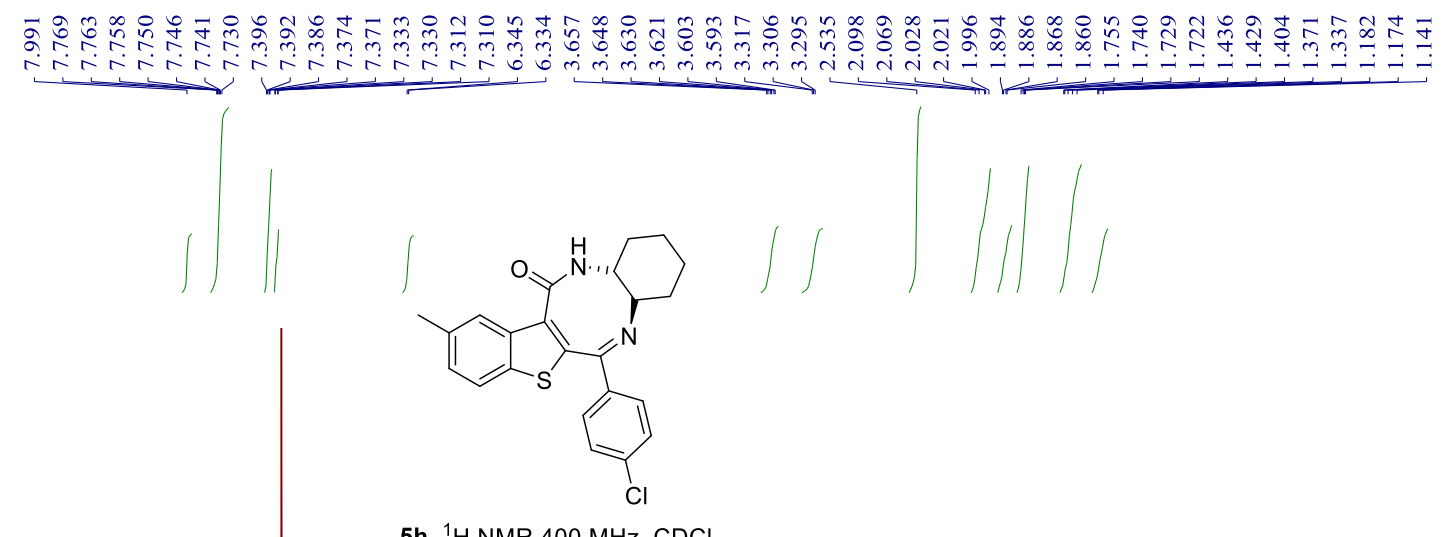

5h, ${ }^{1} \mathrm{H}$ NMR $400 \mathrm{MHz}, \mathrm{CDCl}_{3}$
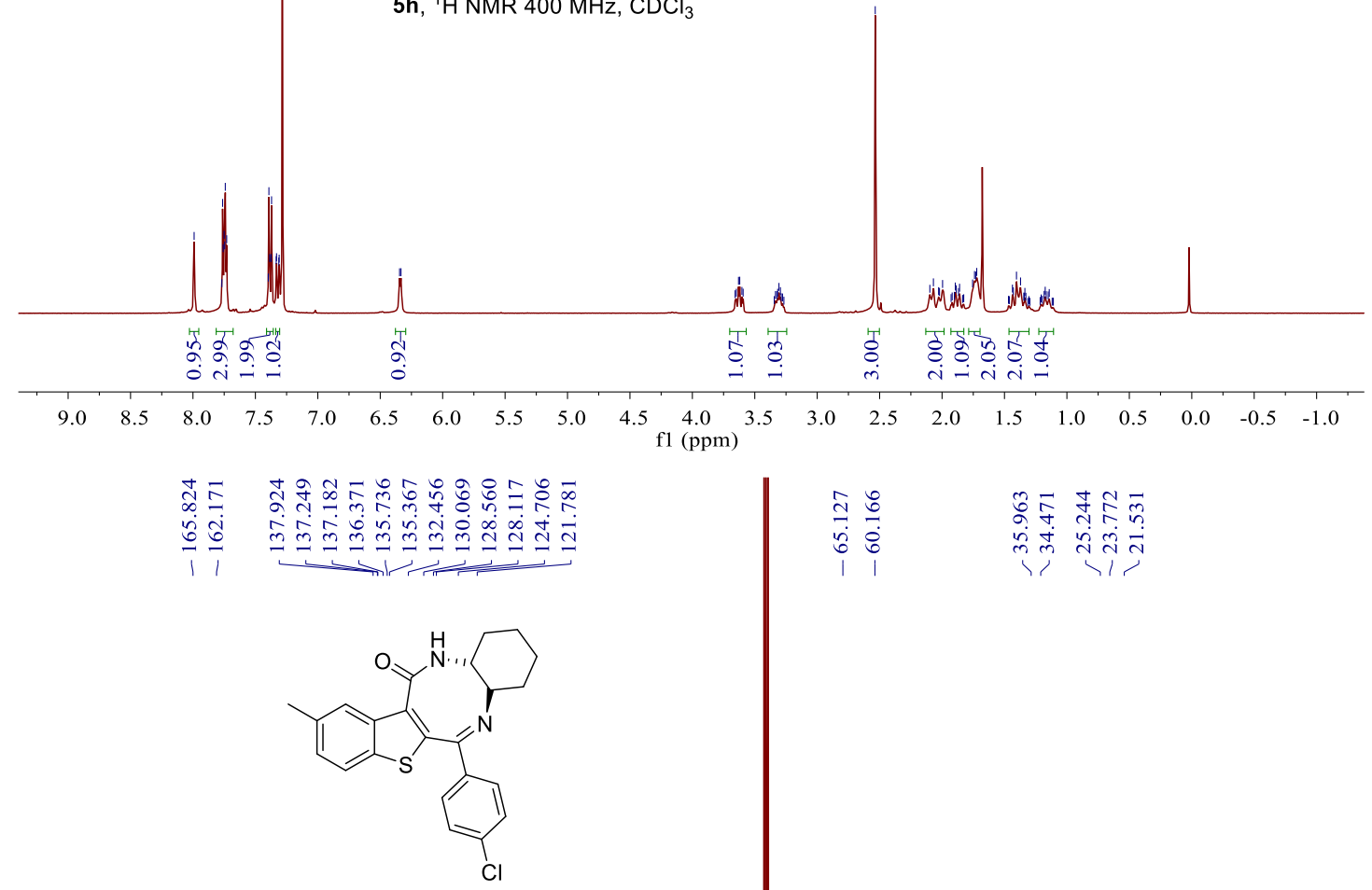

5h, ${ }^{13} \mathrm{C}$ NMR $100 \mathrm{MHz}, \mathrm{CDCl}_{3}$

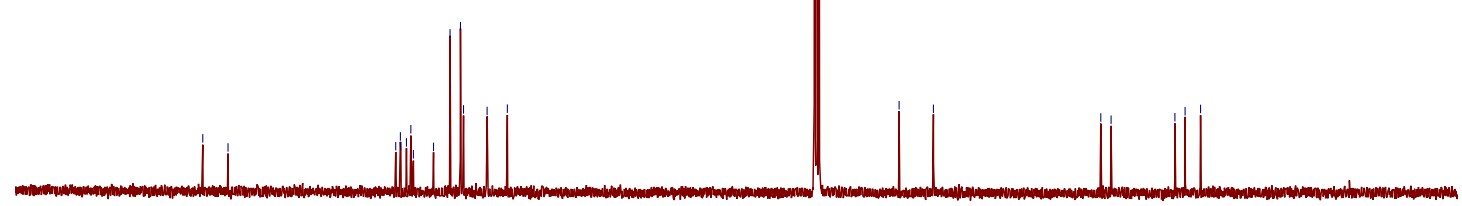

$\begin{array}{lllllllllllllllllllll}190 & 180 & 170 & 160 & 150 & 140 & 130 & 120 & 110 & 100 & 90 & 80 & 70 & 60 & 50 & 40 & 30 & 20 & 10 & 0 & -10\end{array}$ f1 (ppm) 


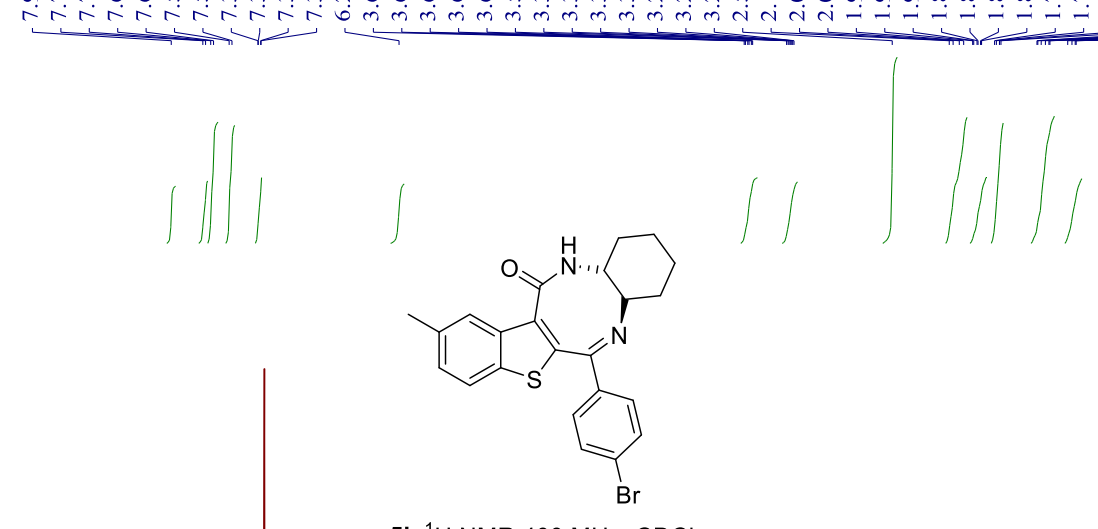

5i, ${ }^{1} \mathrm{H}$ NMR $400 \mathrm{MHz}, \mathrm{CDCl}_{3}$
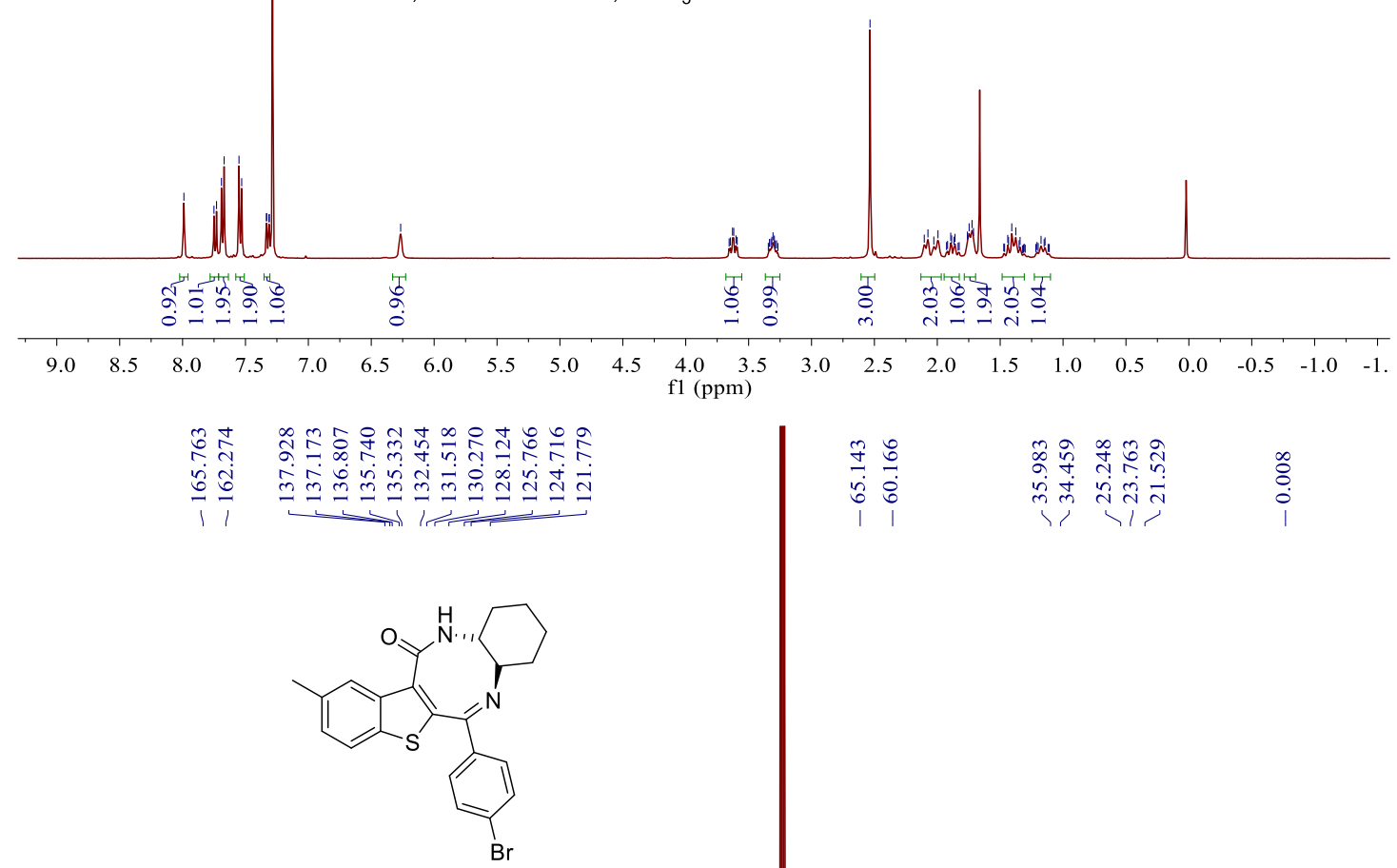

5i, ${ }^{13} \mathrm{C} \mathrm{NMR} 100 \mathrm{MHz}, \mathrm{CDCl}_{3}$

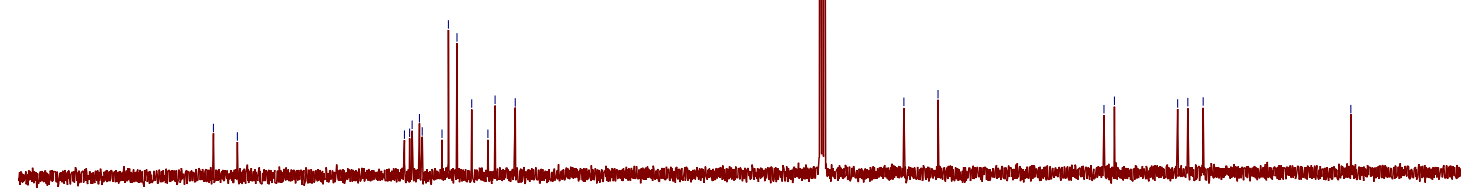

$\begin{array}{lllllllllllllllllllll}190 & 180 & 170 & 160 & 150 & 140 & 130 & 120 & 110 & 100 & \begin{array}{c}90 \\ \mathrm{f} 1(\mathrm{ppm})\end{array} & 80 & 70 & 60 & 50 & 40 & 30 & 20 & 10 & 0 & -10\end{array}$ 


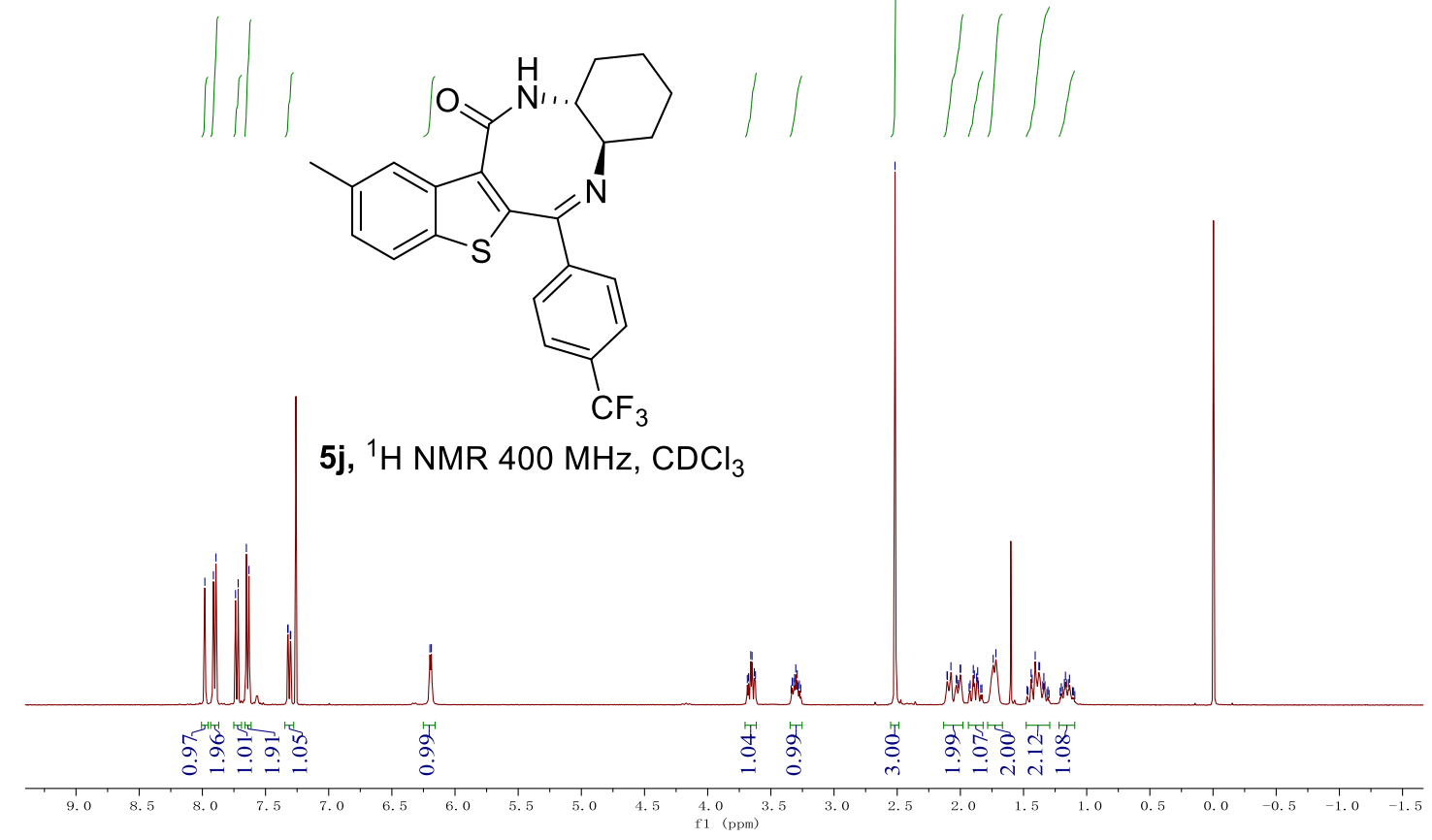

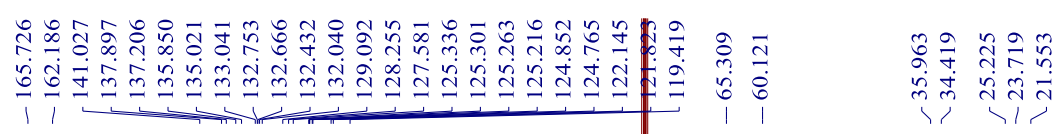<smiles>Cc1ccc2sc3c(c2c1)C(=O)NC1CCCCC1N=C3c1ccc(C(F)(F)F)cc1</smiles>

5j, ${ }^{13} \mathrm{C}$ NMR $100 \mathrm{MHz}, \mathrm{CDCl}_{3}$

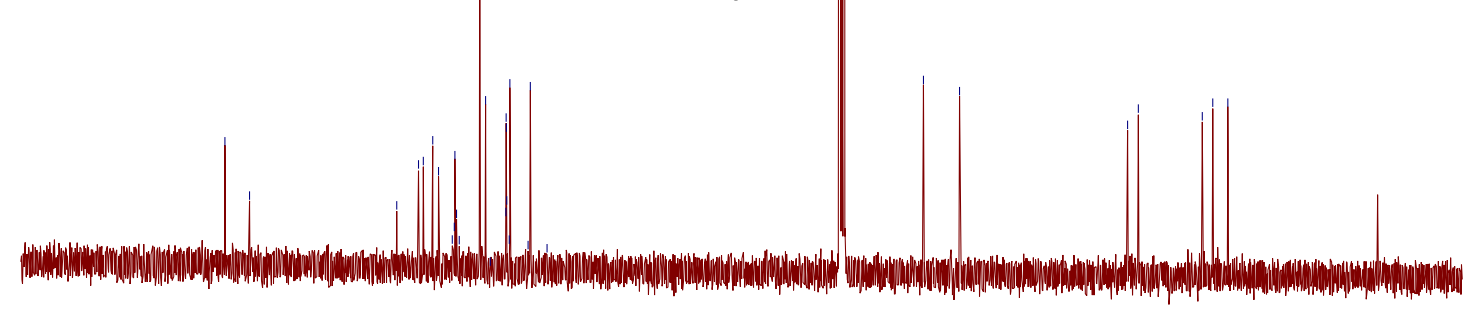

$\begin{array}{lllllllllll}190 & 180 & 170 & 160 & 150 & 140 & 130 & 120 & 110 & 100 & 90 \\ \mathrm{f} 1 & (\mathrm{ppm})\end{array}$ 


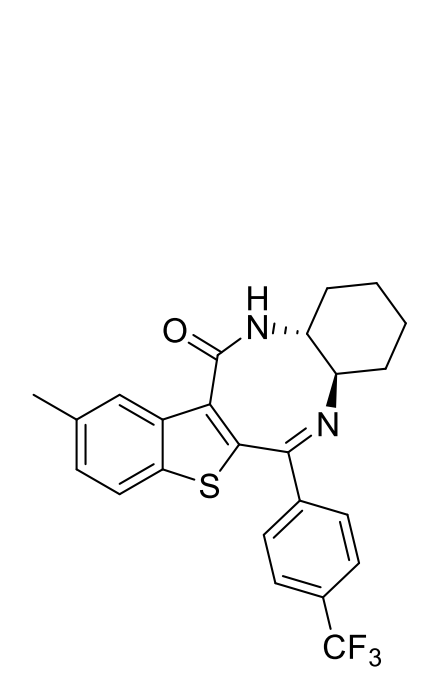

5j, ${ }^{19} \mathrm{~F} \mathrm{NMR} 376 \mathrm{MHz}, \mathrm{CDCl}_{3}$
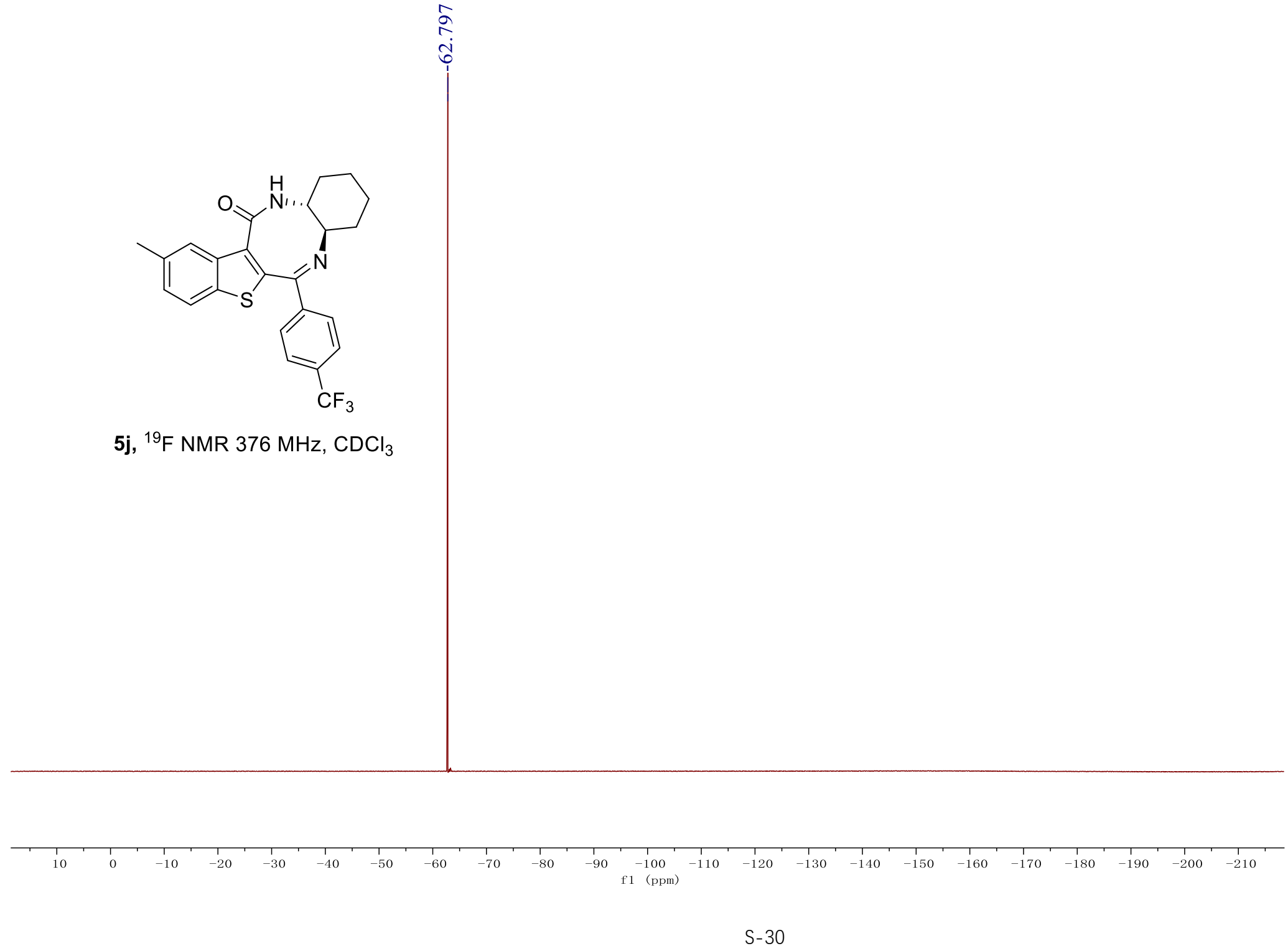


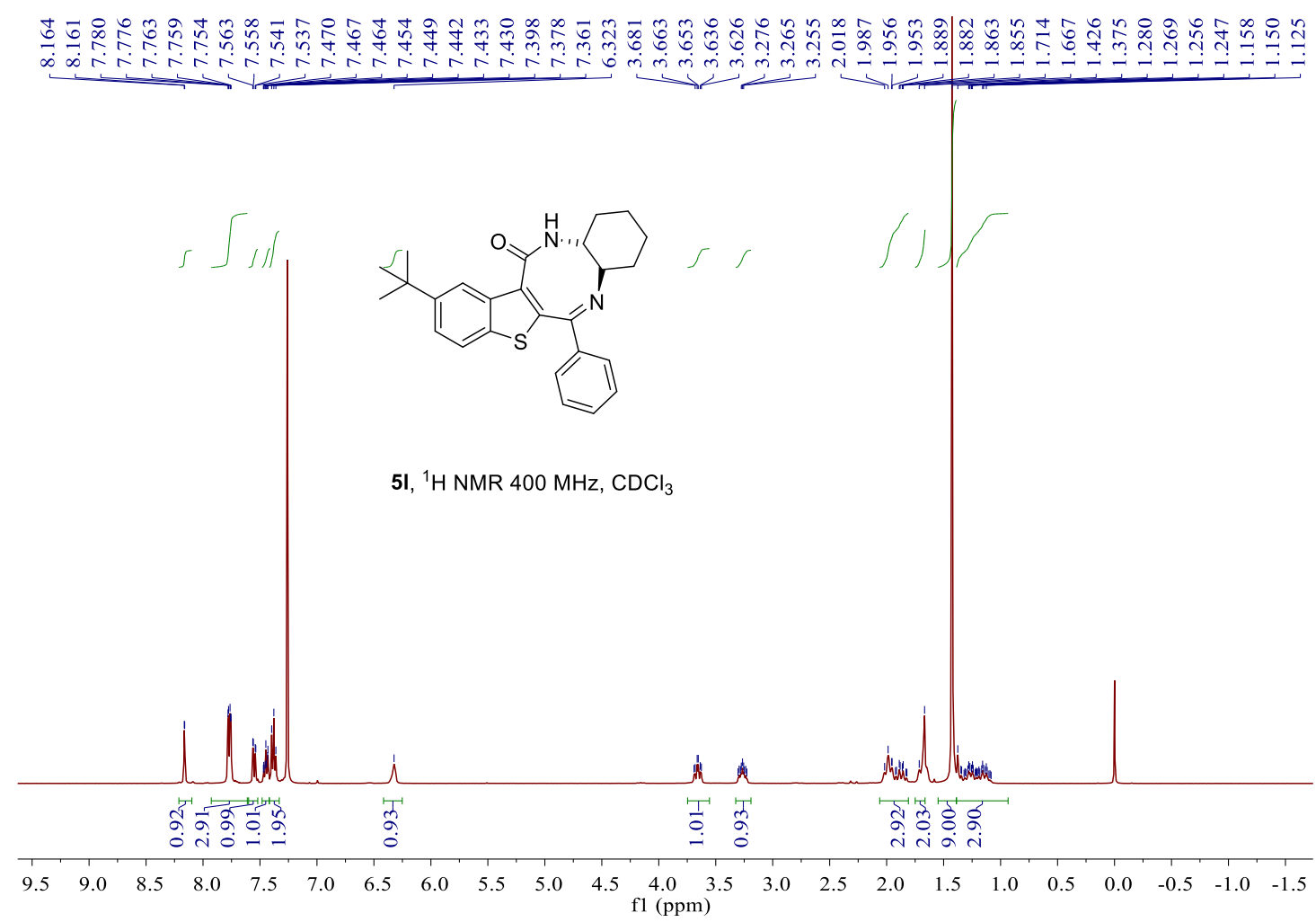

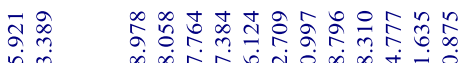

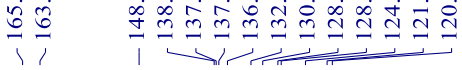

1<smiles>CC(C)(C)c1ccc2sc3c(c2c1)C(=O)NC1CCCCC1N=C3c1ccccc1</smiles>

5I, ${ }^{13} \mathrm{C} \mathrm{NMR} 100 \mathrm{MHz}, \mathrm{CDCl}_{3}$

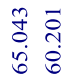

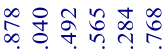

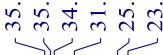

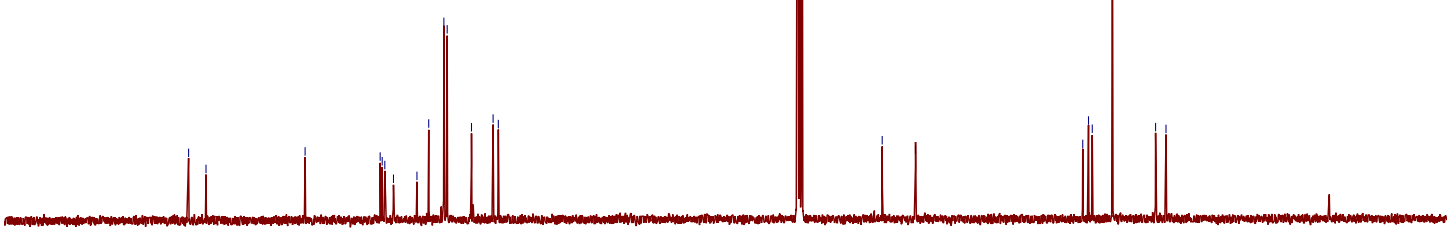

$\begin{array}{lllllllllllllllllllll}190 & 180 & 170 & 160 & 150 & 140 & 130 & 120 & 110 & 100 & 90 & 80 & 70 & 60 & 50 & 40 & 30 & 20 & 10 & 0 & -10\end{array}$ 


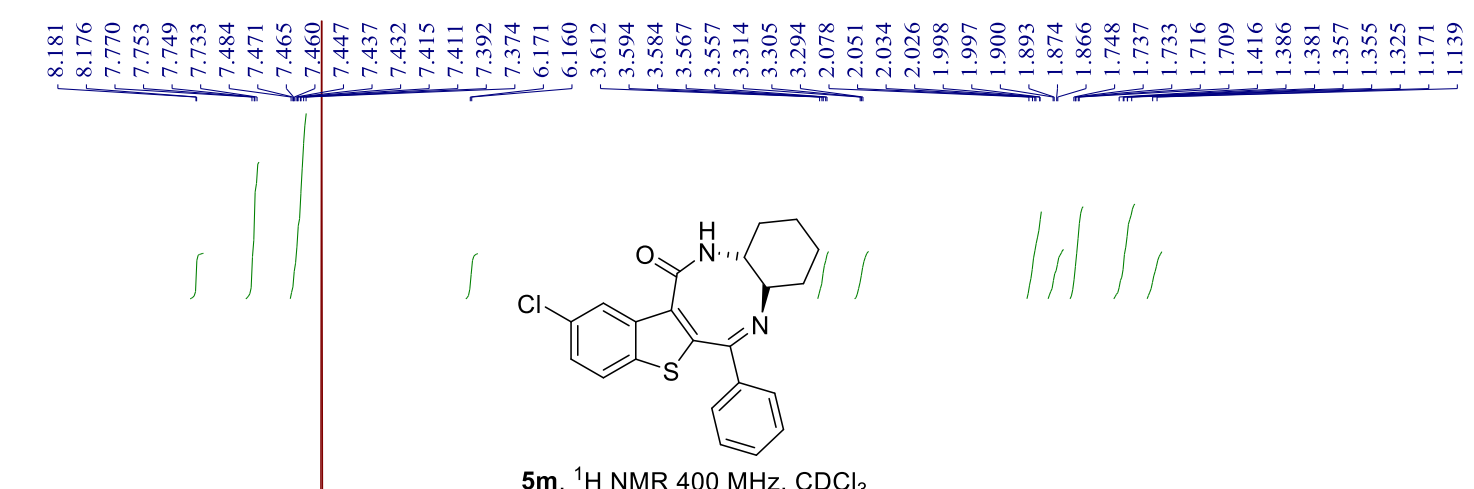

$5 \mathrm{~m},{ }^{1} \mathrm{H}$ NMR $400 \mathrm{MHz}, \mathrm{CDCl}_{3}$

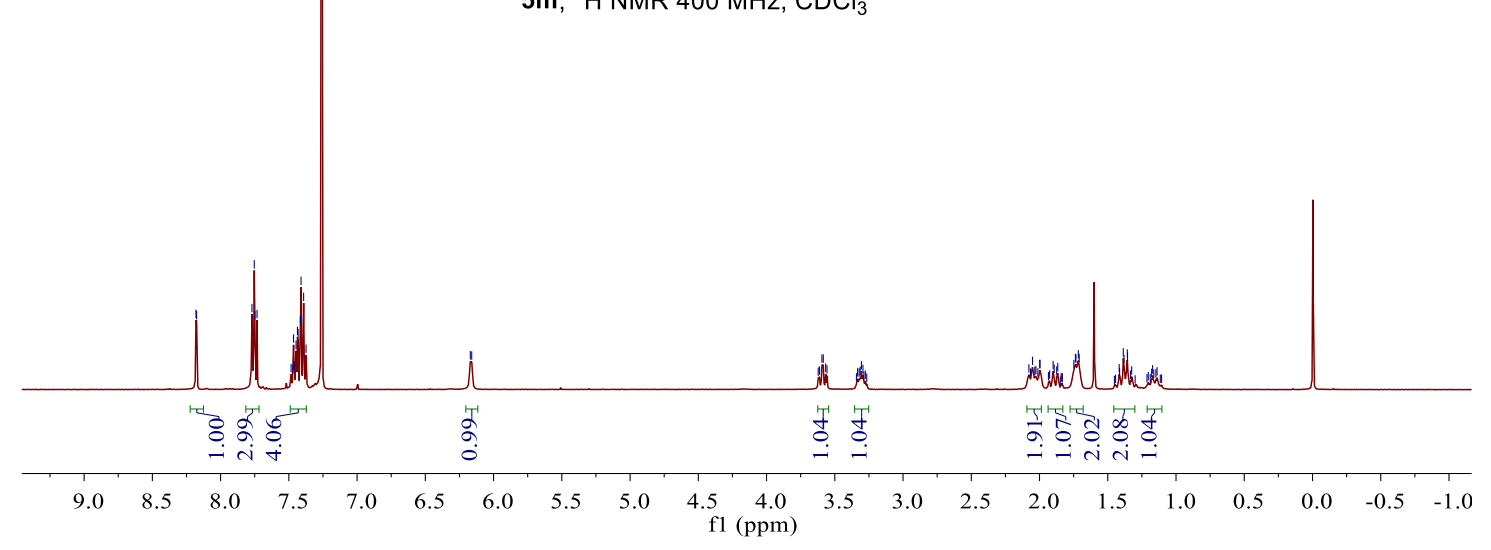

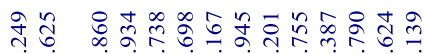

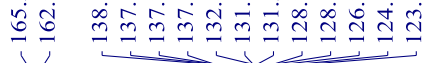

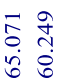

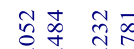

i்

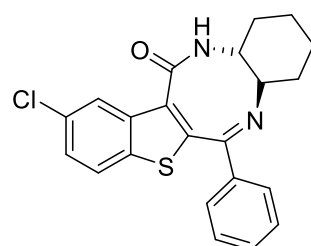

$5 \mathrm{~m},{ }^{13} \mathrm{C}$ NMR $100 \mathrm{MHz}, \mathrm{CDCl}_{3}$

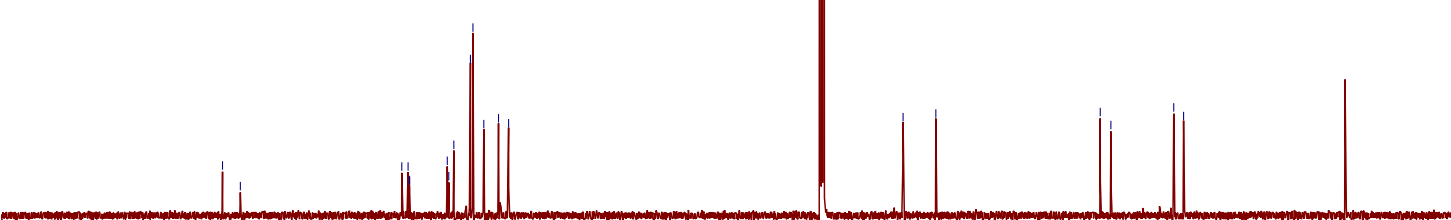

$\begin{array}{lllllllllllllllllllllll}190 & 180 & 170 & 160 & 150 & 140 & 130 & 120 & 110 & 100 & 90 & 80 & 70 & 60 & 50 & 40 & 30 & 20 & 10 & 0 & -10\end{array}$ 


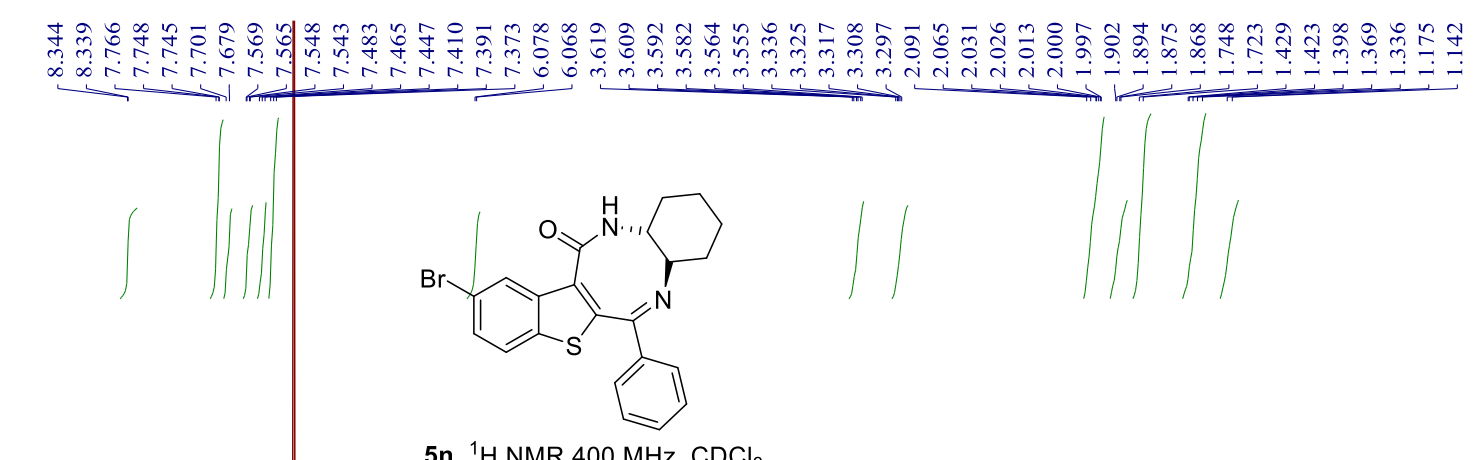

5n, ${ }^{1} \mathrm{H}$ NMR $400 \mathrm{MHz}, \mathrm{CDCl}_{3}$
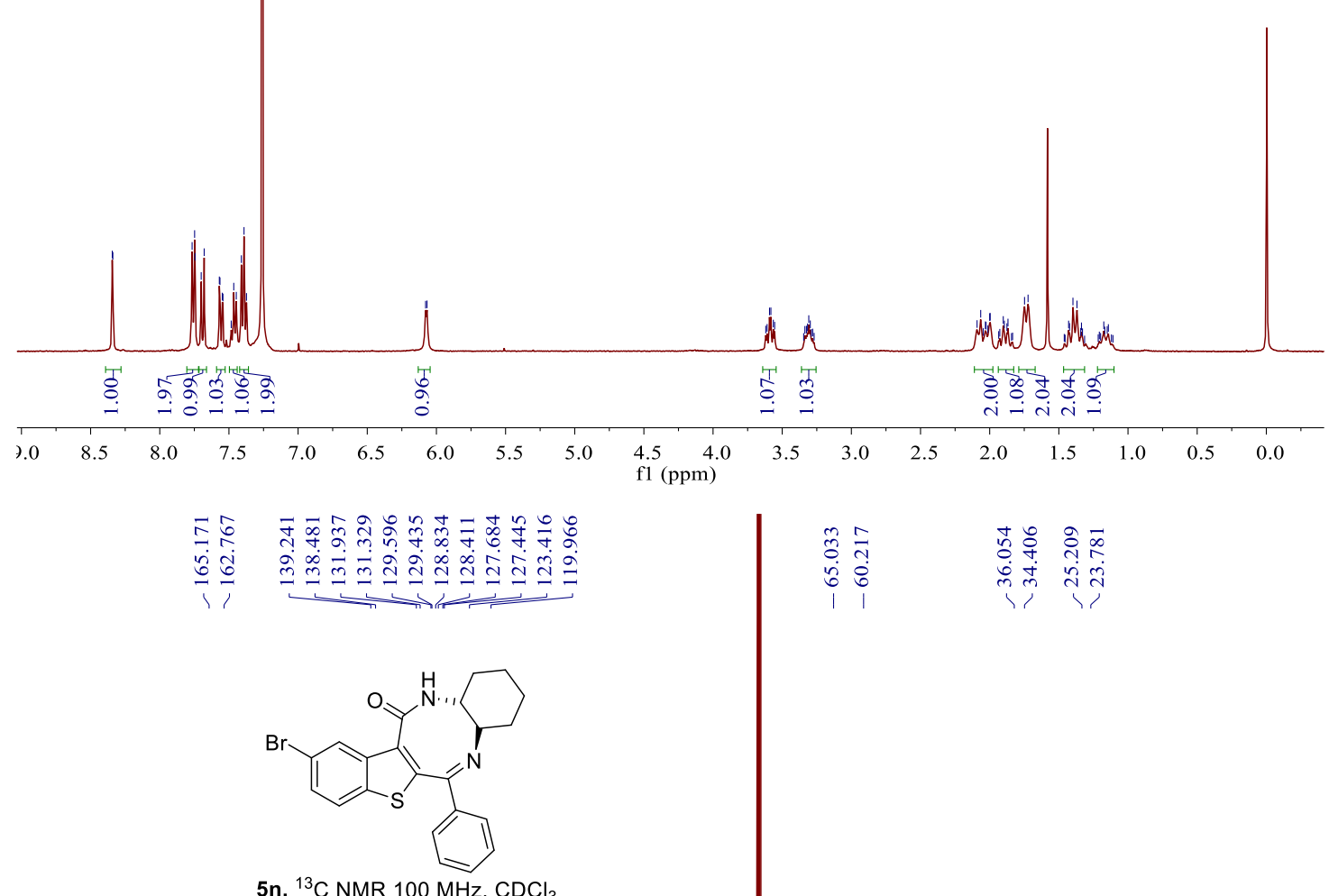

5n, ${ }^{13} \mathrm{C}$ NMR $100 \mathrm{MHz}, \mathrm{CDCl}_{3}$

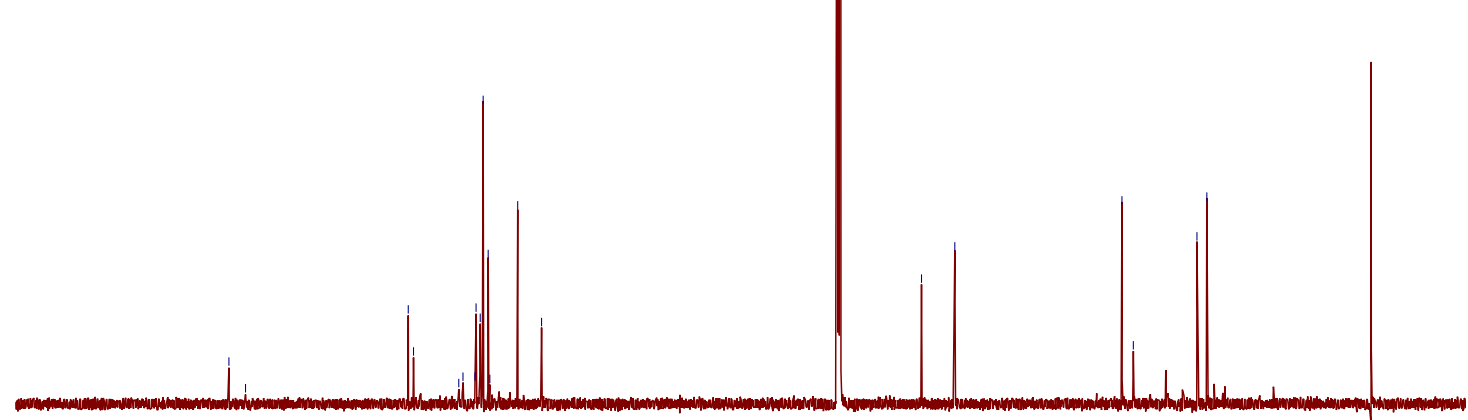

$\begin{array}{llllllllllllllllllll}190 & 180 & 170 & 160 & 150 & 140 & 130 & 120 & 110 & 100 \underset{\mathrm{f} 1(\mathrm{ppm})}{90} & 80 & 70 & 60 & 50 & 40 & 30 & 20 & 10 & 0 & -10\end{array}$ 


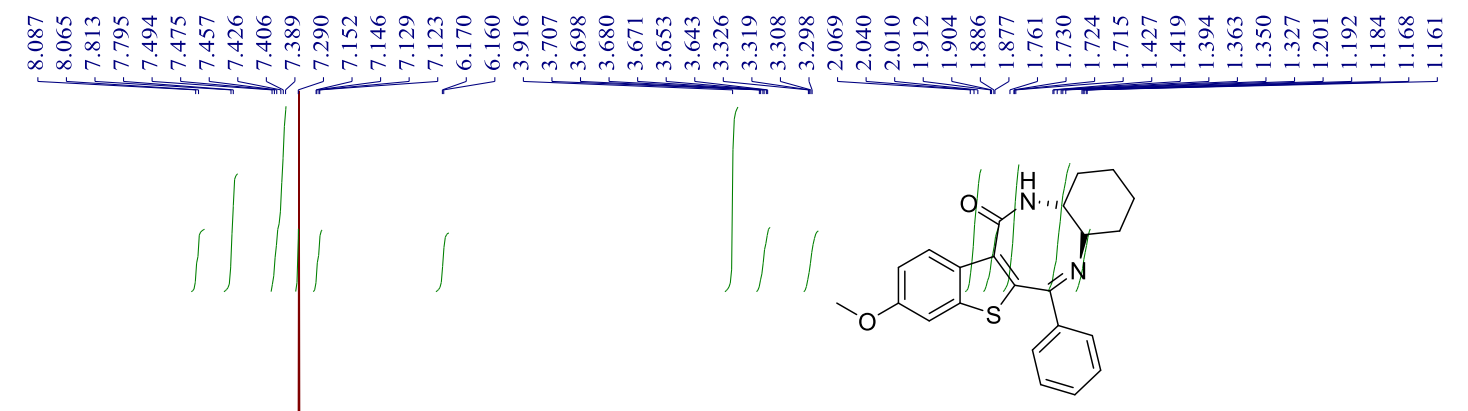

5o, ${ }^{1} \mathrm{H} \mathrm{NMR} 400 \mathrm{MHz}, \mathrm{CDCl}_{3}$

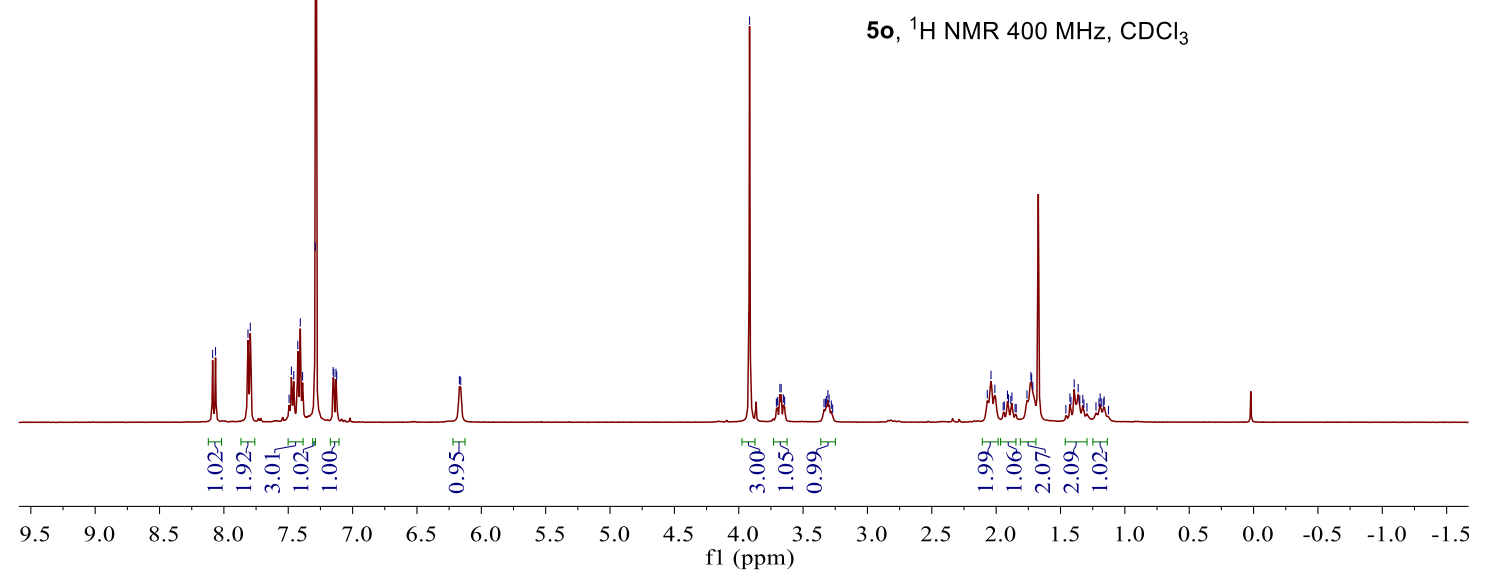

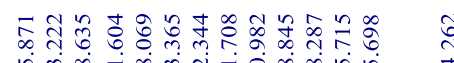

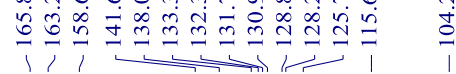

\section{ถึก \\ 60 in}

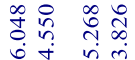

ij<smiles>COc1ccc2c3c(sc2c1)C(c1ccccc1)=N[C@@H]1CCCC[C@H]1NC3=O</smiles>

5o, ${ }^{13} \mathrm{C}$ NMR $100 \mathrm{MHz}, \mathrm{CDCl}_{3}$

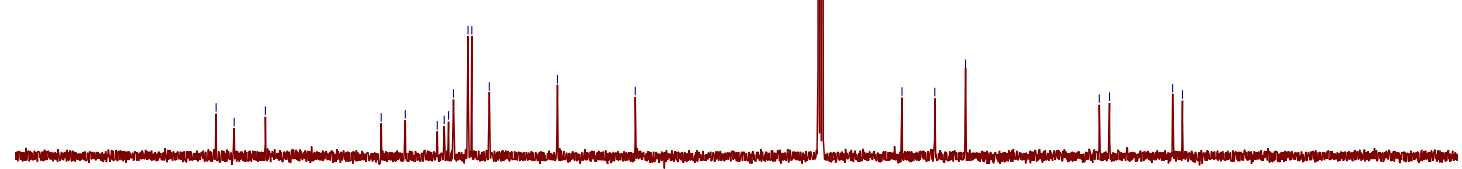

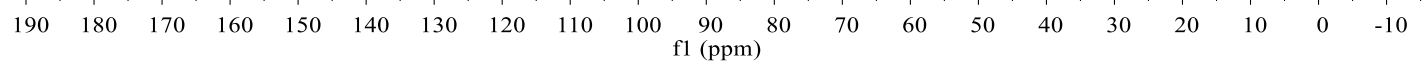




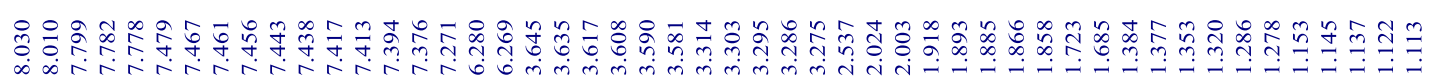

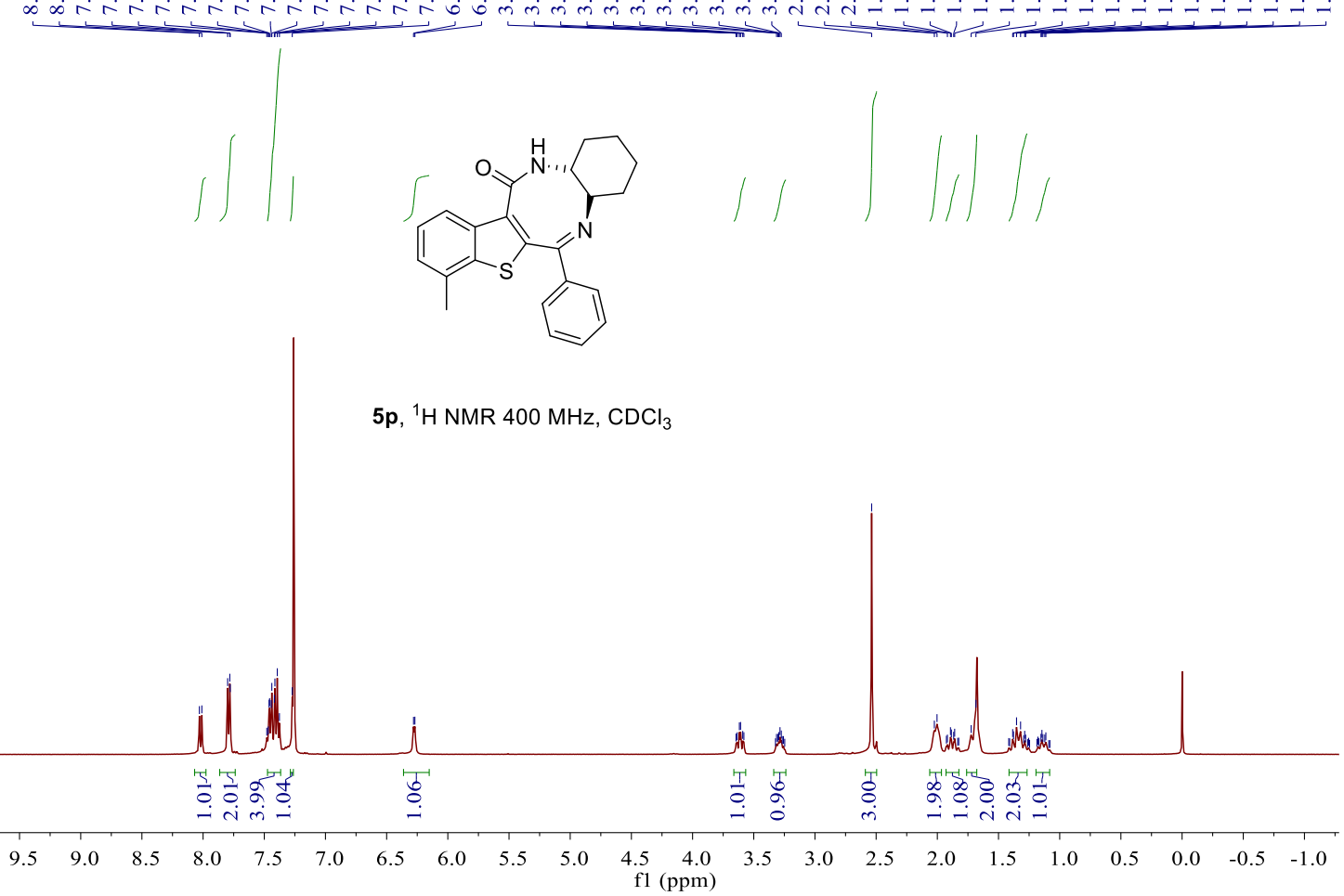

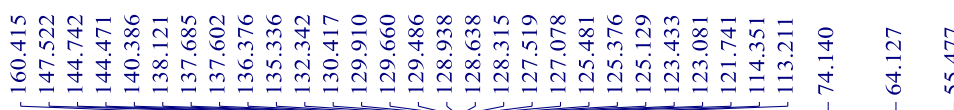

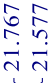

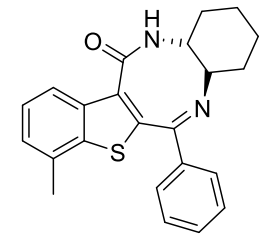

$5 p,{ }^{13} \mathrm{C} \mathrm{NMR} 100 \mathrm{MHz}, \mathrm{CDCl}_{3}$

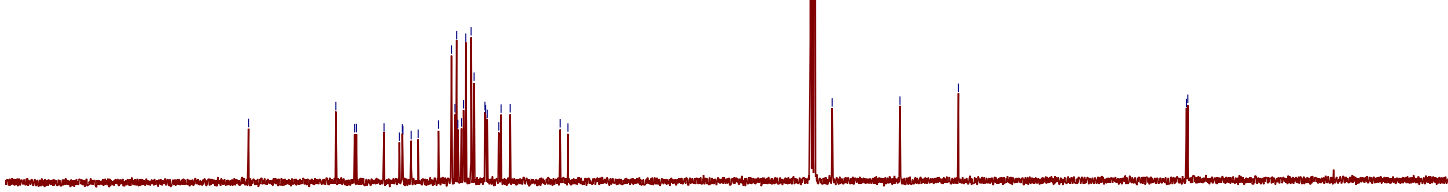

$\begin{array}{llllllllllllllllllll}190 & 180 & 170 & 160 & 150 & 140 & 130 & 120 & 110 & 100 \underset{\mathrm{f} 1}{(\mathrm{ppm})} \mathbf{( \mathrm { ppm } )} & 80 & 70 & 60 & 50 & 40 & 30 & 20 & 10 & 0 & -10\end{array}$ 

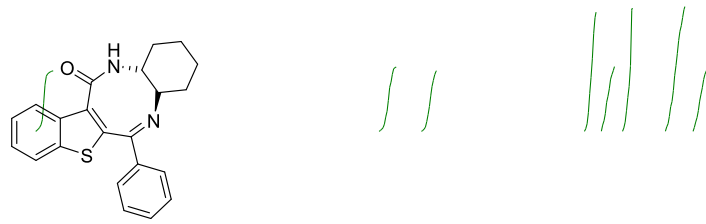

5q, ${ }^{1} \mathrm{H}$ NMR $400 \mathrm{MHz}, \mathrm{CDCl}_{3}$

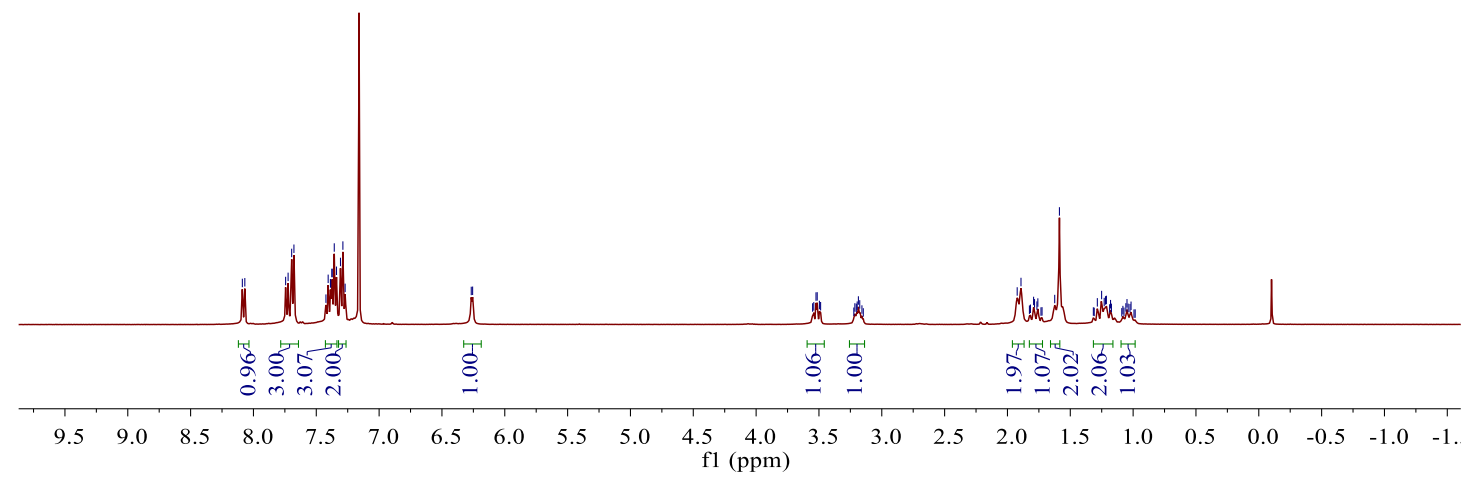

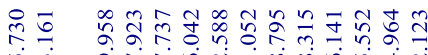

苍苍

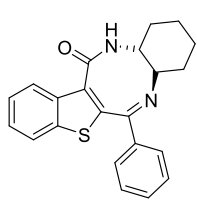

5q, ${ }^{13} \mathrm{C} \mathrm{NMR} 100 \mathrm{MHz}, \mathrm{CDCl}_{3}$

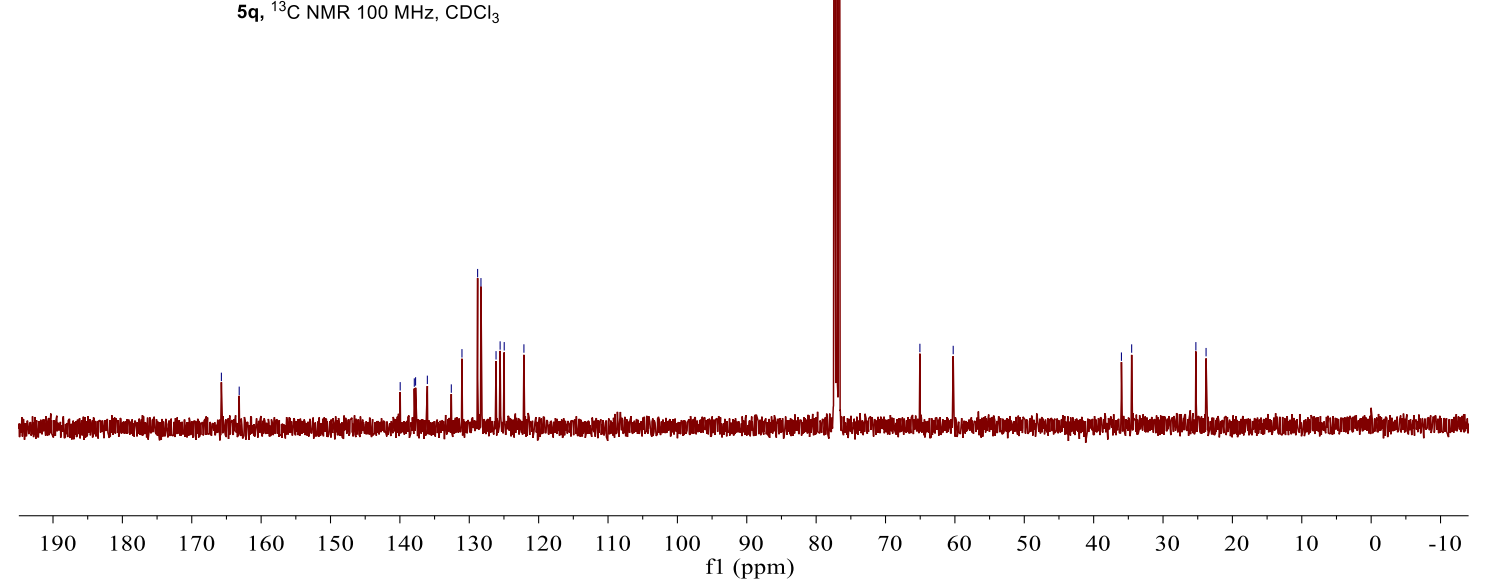



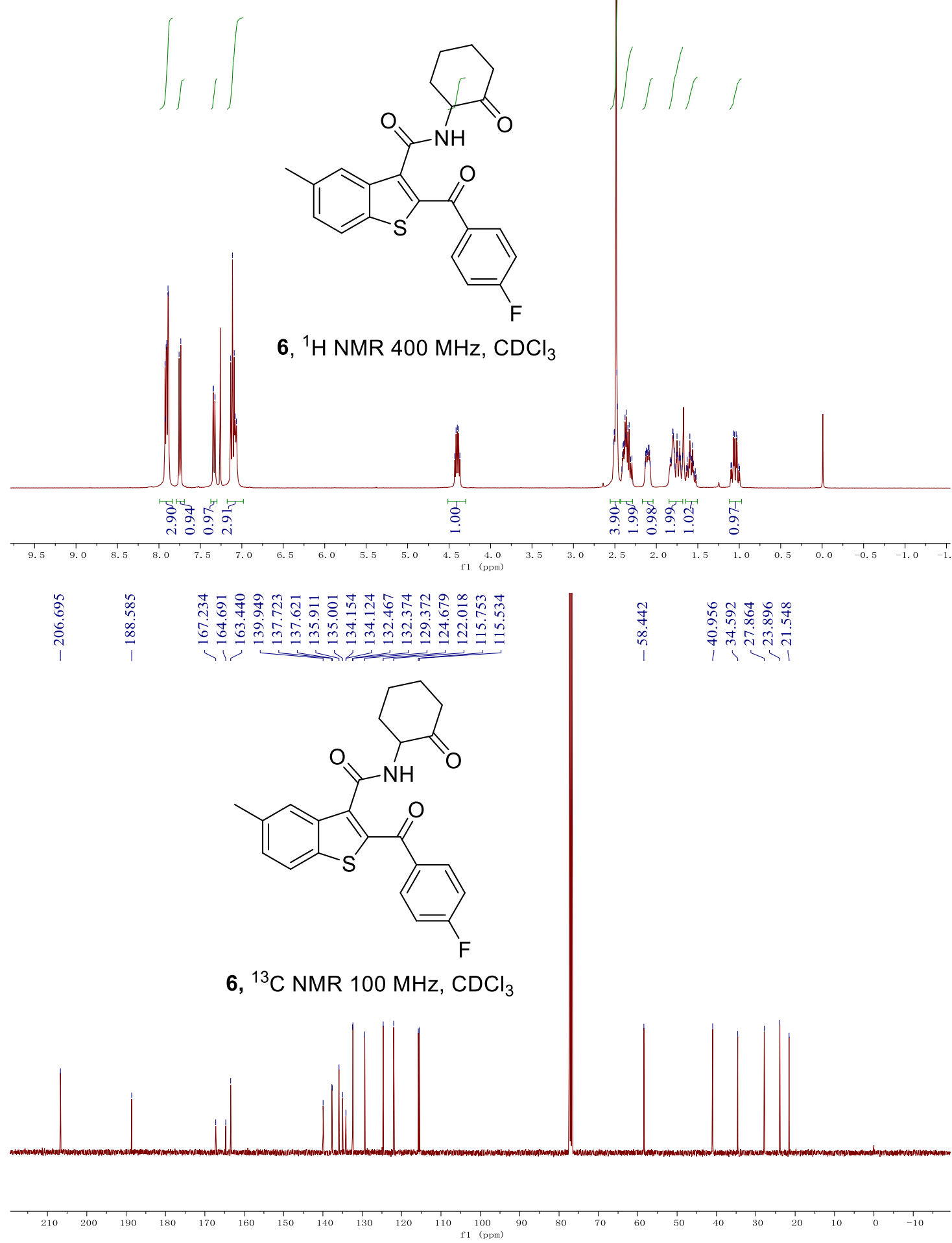


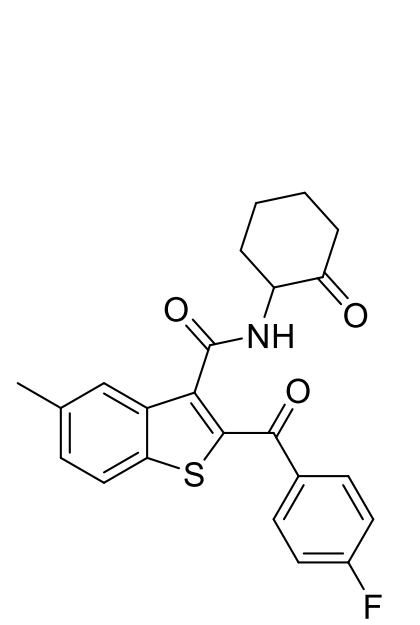

6, ${ }^{19} \mathrm{~F} \mathrm{NMR} 376 \mathrm{MHz}, \mathrm{CDCl}_{3}$

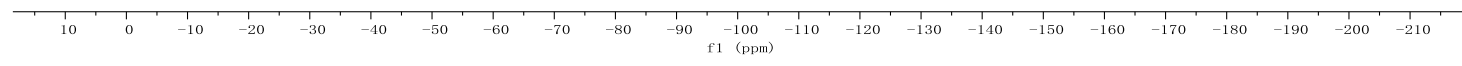

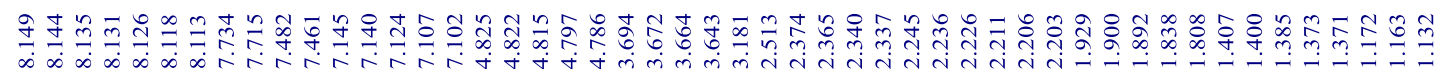

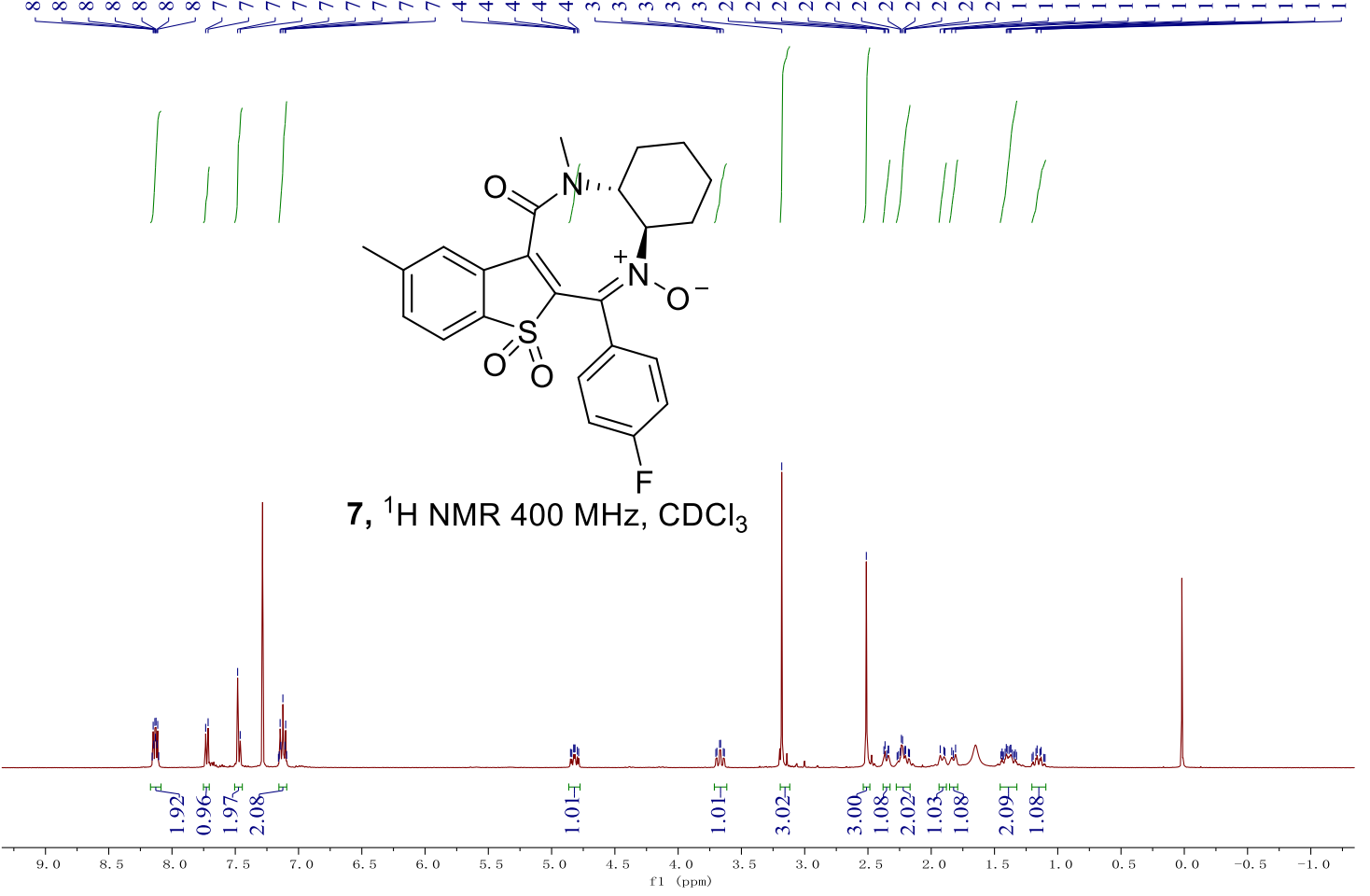



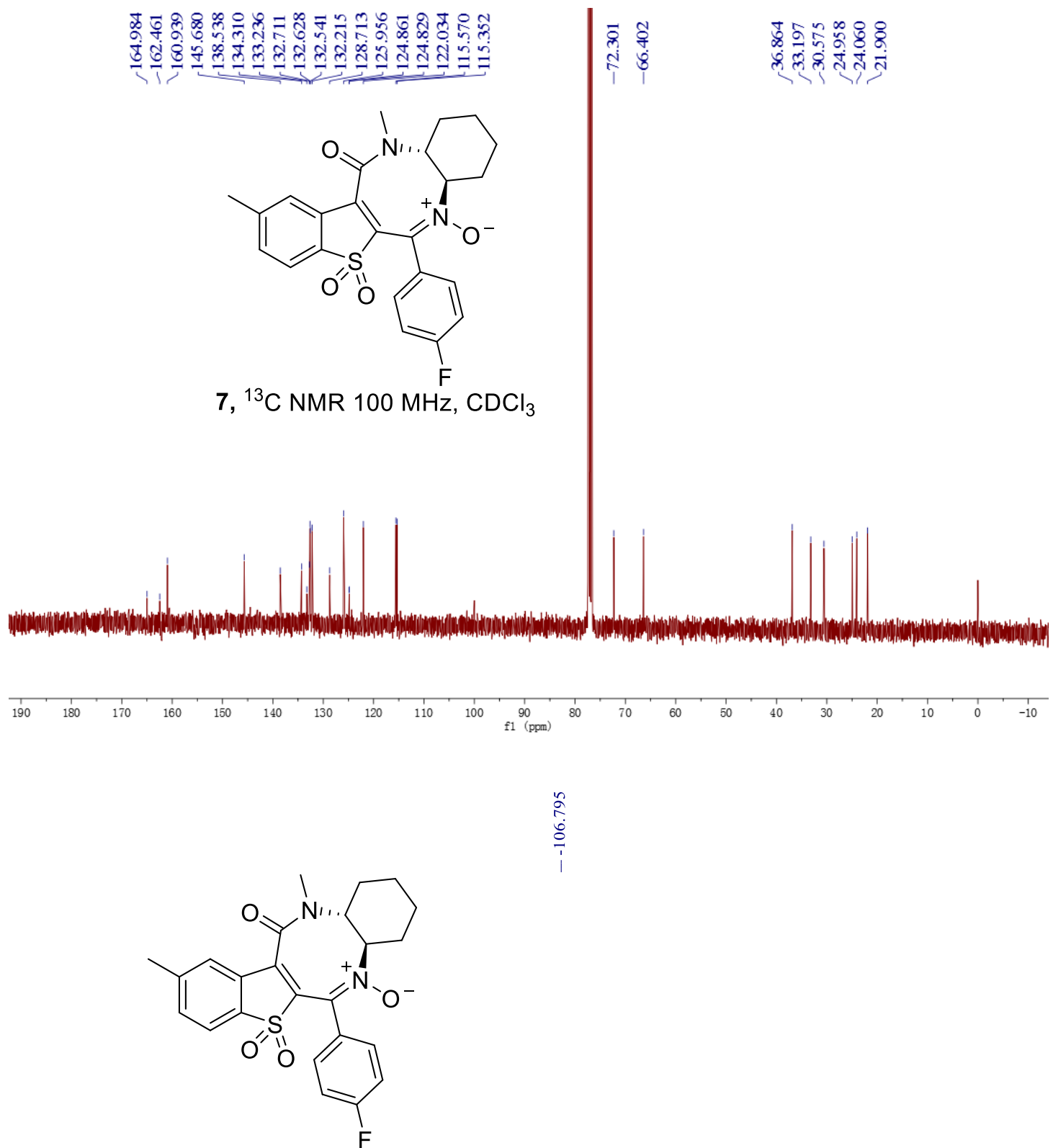

\begin{tabular}{l}
$n$ \\
\multirow{2}{0}{} \\
$\vdots$ \\
$i$
\end{tabular}

7, ${ }^{19} \mathrm{~F}$ NMR $376 \mathrm{MHz}, \mathrm{CDCl}_{3}$

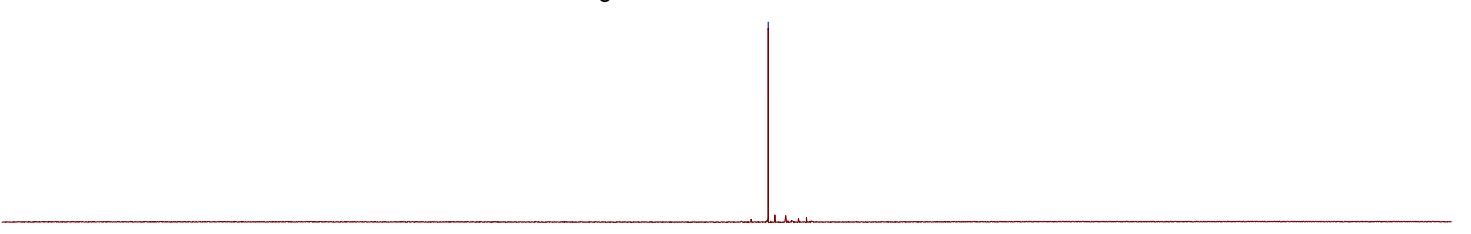




\section{Computational details and archive entries}

Table S1. Cartesian coordinates of key stationary points on the pathway leading to INT-7, optimized at the B3LYP-PCM/6-31+G* level of theory in DMF.

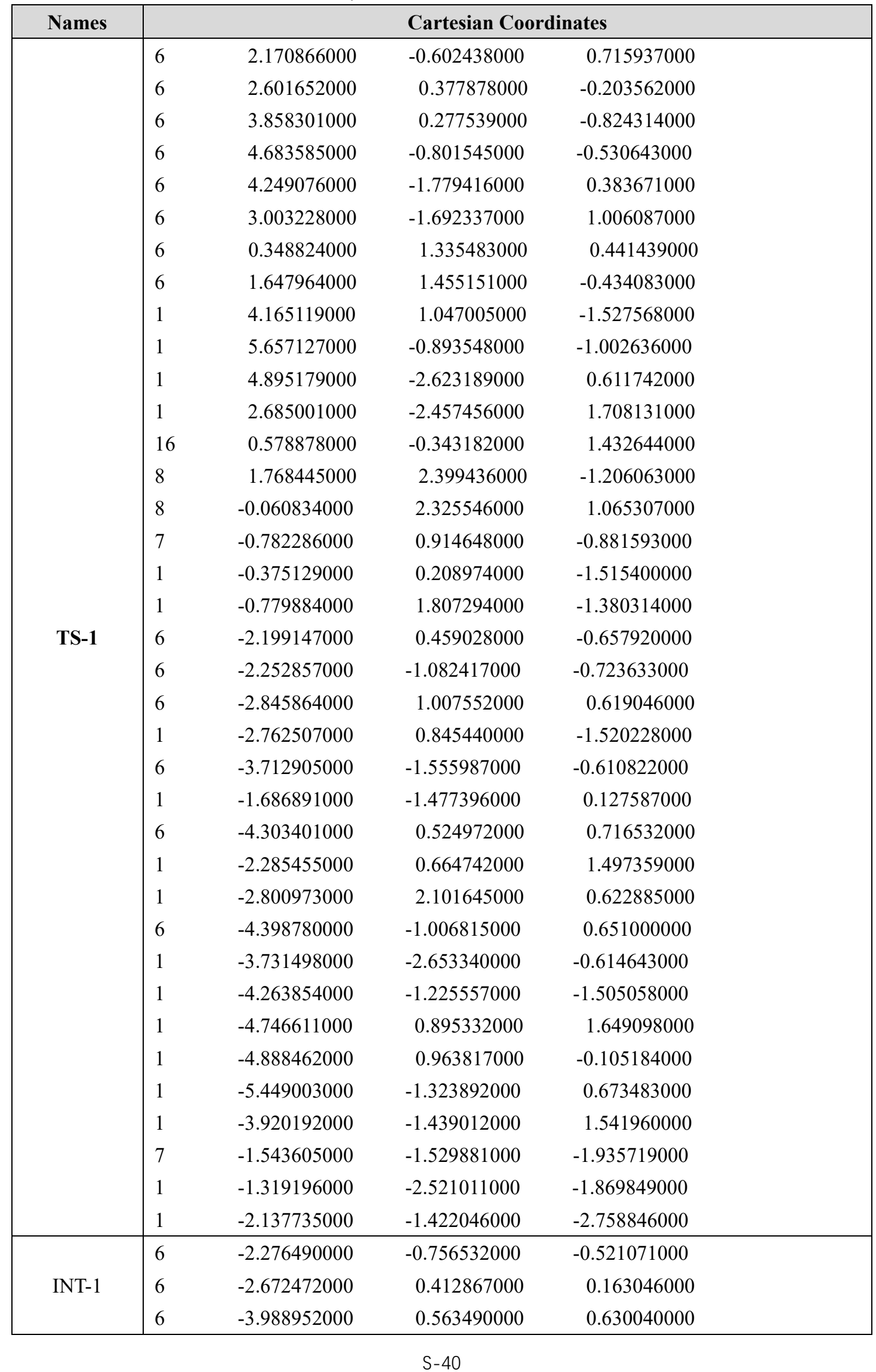




\begin{tabular}{|c|c|c|c|c|}
\hline & 6 & -4.914550000 & -0.450246000 & 0.410979000 \\
\hline & 6 & -4.518792000 & -1.614303000 & -0.274151000 \\
\hline & 6 & -3.213434000 & -1.777356000 & -0.739026000 \\
\hline & 6 & -0.307056000 & 1.085001000 & -0.470833000 \\
\hline & 6 & -1.611297000 & 1.394435000 & 0.347872000 \\
\hline & 1 & -4.264262000 & 1.473267000 & 1.156681000 \\
\hline & 1 & -5.936116000 & -0.348409000 & 0.764715000 \\
\hline & 1 & -5.243129000 & -2.406387000 & -0.445853000 \\
\hline & 1 & -2.926379000 & -2.684721000 & -1.262560000 \\
\hline & 16 & -0.588751000 & -0.830525000 & -1.030202000 \\
\hline & 8 & -1.657327000 & 2.416983000 & 1.025551000 \\
\hline & 8 & -0.027438000 & 1.892860000 & -1.389083000 \\
\hline & 7 & 0.838047000 & 1.026889000 & 0.698624000 \\
\hline & 1 & 0.539005000 & 0.422290000 & 1.486680000 \\
\hline & 1 & 0.820822000 & 1.991990000 & 1.041850000 \\
\hline & 6 & 2.273754000 & 0.604042000 & 0.451520000 \\
\hline & 6 & 2.430839000 & -0.873002000 & 0.877204000 \\
\hline & 6 & 2.803500000 & 0.859758000 & -0.962601000 \\
\hline & 1 & 2.854930000 & 1.216026000 & 1.154384000 \\
\hline & 6 & 3.913756000 & -1.272887000 & 0.796919000 \\
\hline & 1 & 1.862726000 & -1.493324000 & 0.174971000 \\
\hline & 6 & 4.285481000 & 0.448500000 & -1.033899000 \\
\hline & 1 & 2.222822000 & 0.282039000 & -1.691601000 \\
\hline & 1 & 2.682353000 & 1.914569000 & -1.226318000 \\
\hline & 6 & 4.497903000 & -1.010483000 & -0.601301000 \\
\hline & 1 & 4.010901000 & -2.332799000 & 1.063618000 \\
\hline & 1 & 4.478466000 & -0.701448000 & 1.549491000 \\
\hline & 1 & 4.651940000 & 0.600016000 & -2.056470000 \\
\hline & 1 & 4.877667000 & 1.112012000 & -0.386681000 \\
\hline & 1 & 5.567016000 & -1.256637000 & -0.611096000 \\
\hline & 1 & 4.014345000 & -1.679901000 & -1.327718000 \\
\hline & 7 & 1.797807000 & -1.054235000 & 2.194717000 \\
\hline & 1 & 1.598513000 & -2.038122000 & 2.363944000 \\
\hline & 1 & 2.422400000 & -0.749081000 & 2.941865000 \\
\hline \multirow{10}{*}{ TS-2 } & 6 & -2.319590000 & -0.828811000 & -0.386366000 \\
\hline & 6 & -2.661574000 & 0.441971000 & 0.130212000 \\
\hline & 6 & -3.992722000 & 0.757874000 & 0.451593000 \\
\hline & 6 & -4.995005000 & -0.184073000 & 0.249920000 \\
\hline & 6 & -4.658872000 & -1.448106000 & -0.270573000 \\
\hline & 6 & -3.340788000 & -1.775228000 & -0.585835000 \\
\hline & 6 & -0.223277000 & 0.997891000 & -0.415041000 \\
\hline & 6 & -1.547681000 & 1.367125000 & 0.317905000 \\
\hline & 1 & -4.219708000 & 1.742927000 & 0.850958000 \\
\hline & 1 & -6.028165000 & 0.050261000 & 0.489087000 \\
\hline
\end{tabular}




\begin{tabular}{|c|c|c|c|c|}
\hline & 1 & -5.440511000 & -2.186681000 & -0.431065000 \\
\hline & 1 & -3.100570000 & -2.757864000 & -0.982273000 \\
\hline & 16 & -0.617947000 & -1.129752000 & -0.712976000 \\
\hline & 8 & -1.587972000 & 2.429576000 & 0.938830000 \\
\hline & 8 & -0.035328000 & 1.597890000 & -1.503595000 \\
\hline & 7 & 0.880470000 & 1.024750000 & 0.621103000 \\
\hline & 1 & 0.868663000 & 0.101521000 & 1.595867000 \\
\hline & 1 & 0.833617000 & 1.963735000 & 1.023372000 \\
\hline & 6 & 2.299386000 & 0.672922000 & 0.299526000 \\
\hline & 6 & 2.473705000 & -0.788912000 & 0.800624000 \\
\hline & 6 & 2.868856000 & 0.817050000 & -1.112171000 \\
\hline & 1 & 2.891246000 & 1.315080000 & 0.965589000 \\
\hline & 6 & 3.951834000 & -1.147065000 & 0.916664000 \\
\hline & 1 & 1.996355000 & -1.466418000 & 0.086254000 \\
\hline & 6 & 4.364135000 & 0.431253000 & -1.065330000 \\
\hline & 1 & 2.327947000 & 0.167037000 & -1.809454000 \\
\hline & 1 & 2.750779000 & 1.843393000 & -1.471679000 \\
\hline & 6 & 4.609687000 & -0.967537000 & -0.467533000 \\
\hline & 1 & 4.069842000 & -2.179927000 & 1.265131000 \\
\hline & 1 & 4.436621000 & -0.493341000 & 1.655839000 \\
\hline & 1 & 4.783109000 & 0.478136000 & -2.077815000 \\
\hline & 1 & 4.905395000 & 1.179970000 & -0.468516000 \\
\hline & 1 & 5.687164000 & -1.155153000 & -0.388303000 \\
\hline & 1 & 4.208291000 & -1.729463000 & -1.150851000 \\
\hline & 7 & 1.635612000 & -0.847372000 & 2.027730000 \\
\hline & 1 & 1.180571000 & -1.746899000 & 2.173287000 \\
\hline & 1 & 2.141771000 & -0.589785000 & 2.876903000 \\
\hline \multirow{17}{*}{ INT-2 } & 6 & -2.670703000 & -0.822513000 & 0.026739000 \\
\hline & 6 & -2.558266000 & 0.593350000 & 0.112441000 \\
\hline & 6 & -3.629097000 & 1.428274000 & -0.264915000 \\
\hline & 6 & -4.800025000 & 0.896879000 & -0.794140000 \\
\hline & 6 & -4.907700000 & -0.496270000 & -0.930694000 \\
\hline & 6 & -3.870889000 & -1.331722000 & -0.523961000 \\
\hline & 6 & 0.025035000 & 0.839821000 & 0.012934000 \\
\hline & 6 & -1.335170000 & 1.258874000 & 0.608939000 \\
\hline & 1 & -3.512610000 & 2.503789000 & -0.159262000 \\
\hline & 1 & -5.611159000 & 1.549718000 & -1.103870000 \\
\hline & 1 & -5.812440000 & -0.932889000 & -1.347755000 \\
\hline & 1 & -3.983941000 & -2.409434000 & -0.606148000 \\
\hline & 16 & -1.478027000 & -1.944811000 & 0.696377000 \\
\hline & 8 & -1.349033000 & 2.229373000 & 1.365155000 \\
\hline & 8 & 0.138072000 & 0.651451000 & -1.202556000 \\
\hline & 7 & 1.041695000 & 0.804054000 & 0.902580000 \\
\hline & 1 & 0.818851000 & 1.055605000 & 1.858411000 \\
\hline
\end{tabular}




\begin{tabular}{|c|c|c|c|c|}
\hline & 6 & 2.432760000 & 0.450471000 & 0.602016000 \\
\hline & 6 & 2.543319000 & -0.888925000 & -0.150659000 \\
\hline & 6 & 3.192422000 & 1.547714000 & -0.170169000 \\
\hline & 1 & 2.913561000 & 0.327856000 & 1.580288000 \\
\hline & 6 & 4.006247000 & -1.305936000 & -0.332579000 \\
\hline & 1 & 2.049484000 & -0.806121000 & -1.120820000 \\
\hline & 6 & 4.661425000 & 1.150339000 & -0.385113000 \\
\hline & 1 & 2.698131000 & 1.715141000 & -1.134585000 \\
\hline & 1 & 3.123170000 & 2.481556000 & 0.398881000 \\
\hline & 6 & 4.781618000 & -0.210922000 & -1.086114000 \\
\hline & 1 & 4.053826000 & -2.251569000 & -0.886107000 \\
\hline & 1 & 4.468172000 & -1.478937000 & 0.649515000 \\
\hline & 1 & 5.167432000 & 1.923909000 & -0.974754000 \\
\hline & 1 & 5.174080000 & 1.108264000 & 0.586704000 \\
\hline & 1 & 5.833454000 & -0.507706000 & -1.168130000 \\
\hline & 1 & 4.393151000 & -0.133306000 & -2.111072000 \\
\hline & 7 & 1.765330000 & -1.954126000 & 0.589084000 \\
\hline & 1 & 1.896603000 & -2.870899000 & 0.149308000 \\
\hline & 1 & 2.065954000 & -2.043895000 & 1.565540000 \\
\hline & 1 & 0.725365000 & -1.766798000 & 0.591029000 \\
\hline \multirow{24}{*}{ TS-3 } & 6 & 0.236103000 & 2.628844000 & 0.092956000 \\
\hline & 6 & -0.961548000 & 3.391568000 & 0.133070000 \\
\hline & 6 & -0.940589000 & 4.724858000 & 0.580839000 \\
\hline & 6 & 0.236433000 & 5.304140000 & 1.045304000 \\
\hline & 6 & 1.410954000 & 4.541319000 & 1.049194000 \\
\hline & 6 & 1.414149000 & 3.227395000 & 0.579520000 \\
\hline & 6 & -2.706040000 & 1.475154000 & 0.272576000 \\
\hline & 6 & -2.273320000 & 2.845317000 & -0.289341000 \\
\hline & 1 & -1.869437000 & 5.288553000 & 0.580806000 \\
\hline & 1 & 0.239532000 & 6.328718000 & 1.405513000 \\
\hline & 1 & 2.338240000 & 4.973741000 & 1.417285000 \\
\hline & 1 & 2.337509000 & 2.659047000 & 0.583919000 \\
\hline & 16 & 0.259487000 & 1.025807000 & -0.679574000 \\
\hline & 8 & -3.089508000 & 3.478251000 & -0.951560000 \\
\hline & 8 & -2.505704000 & 1.202181000 & 1.462790000 \\
\hline & 7 & -3.365718000 & 0.675185000 & -0.588550000 \\
\hline & 1 & -3.495279000 & 1.002399000 & -1.540143000 \\
\hline & 6 & 1.754987000 & -2.545487000 & 0.061431000 \\
\hline & 6 & 1.288229000 & -3.759426000 & 0.572174000 \\
\hline & 6 & 1.335757000 & -4.008983000 & 1.949171000 \\
\hline & 6 & 1.849594000 & -3.037110000 & 2.814865000 \\
\hline & 6 & 2.313775000 & -1.825250000 & 2.304246000 \\
\hline & 6 & 2.273497000 & -1.563754000 & 0.924635000 \\
\hline & 1 & 1.720211000 & -2.383076000 & -1.010290000 \\
\hline
\end{tabular}




\begin{tabular}{|c|c|c|c|c|}
\hline & 1 & 0.899951000 & -4.514320000 & -0.106119000 \\
\hline & 1 & 0.972676000 & -4.954498000 & 2.342843000 \\
\hline & 1 & 1.885711000 & -3.223344000 & 3.884626000 \\
\hline & 1 & 2.707913000 & -1.063401000 & 2.968818000 \\
\hline & 6 & 2.743129000 & -0.220684000 & 0.457232000 \\
\hline & 8 & 3.267053000 & 0.574412000 & 1.238780000 \\
\hline & 6 & 2.573108000 & 0.166965000 & -0.974690000 \\
\hline & 1 & 2.094576000 & -0.458262000 & -1.707781000 \\
\hline & 1 & 2.874905000 & 1.158618000 & -1.266966000 \\
\hline & 35 & 4.727943000 & -0.715301000 & -1.621325000 \\
\hline & 6 & -3.905818000 & -0.632832000 & -0.217358000 \\
\hline & 6 & -5.129103000 & -0.967953000 & -1.086494000 \\
\hline & 6 & -2.846891000 & -1.749087000 & -0.340150000 \\
\hline & 1 & -4.218091000 & -0.564464000 & 0.831429000 \\
\hline & 6 & -5.711417000 & -2.345801000 & -0.738175000 \\
\hline & 1 & -4.836058000 & -0.948716000 & -2.146074000 \\
\hline & 1 & -5.879366000 & -0.182100000 & -0.945184000 \\
\hline & 6 & -3.420817000 & -3.121936000 & 0.025864000 \\
\hline & 1 & -2.446269000 & -1.763807000 & -1.358713000 \\
\hline & 6 & -4.646361000 & -3.446204000 & -0.842888000 \\
\hline & 1 & -6.550296000 & -2.564714000 & -1.408746000 \\
\hline & 1 & -6.118558000 & -2.323228000 & 0.282363000 \\
\hline & 1 & -2.647363000 & -3.888492000 & -0.103924000 \\
\hline & 1 & -3.706325000 & -3.126233000 & 1.086963000 \\
\hline & 1 & -5.061115000 & -4.411628000 & -0.532177000 \\
\hline & 1 & -4.331632000 & -3.557994000 & -1.889913000 \\
\hline & 7 & -1.659331000 & -1.435952000 & 0.546970000 \\
\hline & 1 & -1.949466000 & -1.221881000 & 1.507173000 \\
\hline & 1 & -1.004702000 & -2.227684000 & 0.580664000 \\
\hline & 1 & -1.113331000 & -0.610316000 & 0.210266000 \\
\hline \multirow{14}{*}{ INT-3 } & 6 & 0.400587000 & 2.664575000 & -0.008391000 \\
\hline & 6 & -0.817087000 & 3.387406000 & 0.077938000 \\
\hline & 6 & -0.809812000 & 4.705665000 & 0.564530000 \\
\hline & 6 & 0.366884000 & 5.296424000 & 1.017285000 \\
\hline & 6 & 1.556106000 & 4.565260000 & 0.968752000 \\
\hline & 6 & 1.578496000 & 3.266855000 & 0.452652000 \\
\hline & 6 & -2.565209000 & 1.462584000 & 0.231409000 \\
\hline & 6 & -2.139685000 & 2.840326000 & -0.326505000 \\
\hline & 1 & -1.750053000 & 5.247244000 & 0.607128000 \\
\hline & 1 & 0.354529000 & 6.308761000 & 1.409454000 \\
\hline & 1 & 2.483089000 & 5.004479000 & 1.327075000 \\
\hline & 1 & 2.517563000 & 2.729466000 & 0.423223000 \\
\hline & 16 & 0.378814000 & 1.087823000 & -0.864557000 \\
\hline & 8 & -2.959906000 & 3.481533000 & -0.969746000 \\
\hline
\end{tabular}




\begin{tabular}{|c|c|c|c|c|}
\hline & 8 & -2.264658000 & 1.138214000 & 1.385566000 \\
\hline & 7 & -3.303640000 & 0.709085000 & -0.606304000 \\
\hline & 1 & -3.533169000 & 1.100236000 & -1.514618000 \\
\hline & 6 & 1.657243000 & -2.467642000 & 0.010934000 \\
\hline & 6 & 1.211035000 & -3.714120000 & 0.459557000 \\
\hline & 6 & 1.108393000 & -3.973319000 & 1.831867000 \\
\hline & 6 & 1.460897000 & -2.983209000 & 2.756293000 \\
\hline & 6 & 1.913135000 & -1.741986000 & 2.309137000 \\
\hline & 6 & 2.021331000 & -1.471065000 & 0.933857000 \\
\hline & 1 & 1.753644000 & -2.296285000 & -1.055537000 \\
\hline & 1 & 0.955693000 & -4.485366000 & -0.261931000 \\
\hline & 1 & 0.759446000 & -4.942842000 & 2.176752000 \\
\hline & 1 & 1.378432000 & -3.177667000 & 3.821984000 \\
\hline & 1 & 2.187897000 & -0.968486000 & 3.019323000 \\
\hline & 6 & 2.510767000 & -0.117505000 & 0.523385000 \\
\hline & 8 & 3.141277000 & 0.586024000 & 1.306852000 \\
\hline & 6 & 2.108229000 & 0.445576000 & -0.834751000 \\
\hline & 1 & 2.108759000 & -0.314798000 & -1.614691000 \\
\hline & 1 & 2.799486000 & 1.231821000 & -1.136191000 \\
\hline & 35 & 5.077095000 & -1.035954000 & -1.421052000 \\
\hline & 6 & -3.944777000 & -0.546653000 & -0.205164000 \\
\hline & 6 & -5.236432000 & -0.756679000 & -1.014198000 \\
\hline & 6 & -3.008546000 & -1.758931000 & -0.385419000 \\
\hline & 1 & -4.196729000 & -0.453618000 & 0.857878000 \\
\hline & 6 & -5.930670000 & -2.074167000 & -0.641022000 \\
\hline & 1 & -4.992862000 & -0.759691000 & -2.086165000 \\
\hline & 1 & -5.899964000 & 0.096292000 & -0.834357000 \\
\hline & 6 & -3.691254000 & -3.075092000 & 0.000628000 \\
\hline & 1 & -2.654597000 & -1.798373000 & -1.420243000 \\
\hline & 6 & -4.984824000 & -3.270806000 & -0.807013000 \\
\hline & 1 & -6.819399000 & -2.203752000 & -1.268995000 \\
\hline & 1 & -6.281969000 & -2.022547000 & 0.398896000 \\
\hline & 1 & -3.003317000 & -3.910700000 & -0.173402000 \\
\hline & 1 & -3.926073000 & -3.063385000 & 1.073718000 \\
\hline & 1 & -5.473777000 & -4.194647000 & -0.478873000 \\
\hline & 1 & -4.736321000 & -3.402738000 & -1.869124000 \\
\hline & 7 & -1.754073000 & -1.572892000 & 0.451240000 \\
\hline & 1 & -1.974324000 & -1.407102000 & 1.439431000 \\
\hline & 1 & -1.142369000 & -2.397768000 & 0.398550000 \\
\hline & 1 & -1.191518000 & -0.765850000 & 0.139963000 \\
\hline \multirow{4}{*}{ INT-3-1 } & 6 & 3.077136000 & -0.608910000 & 0.445195000 \\
\hline & 6 & 2.583944000 & -1.837308000 & -0.041514000 \\
\hline & 6 & 3.431679000 & -2.685723000 & -0.776712000 \\
\hline & 6 & 4.731144000 & -2.295490000 & -1.087984000 \\
\hline
\end{tabular}




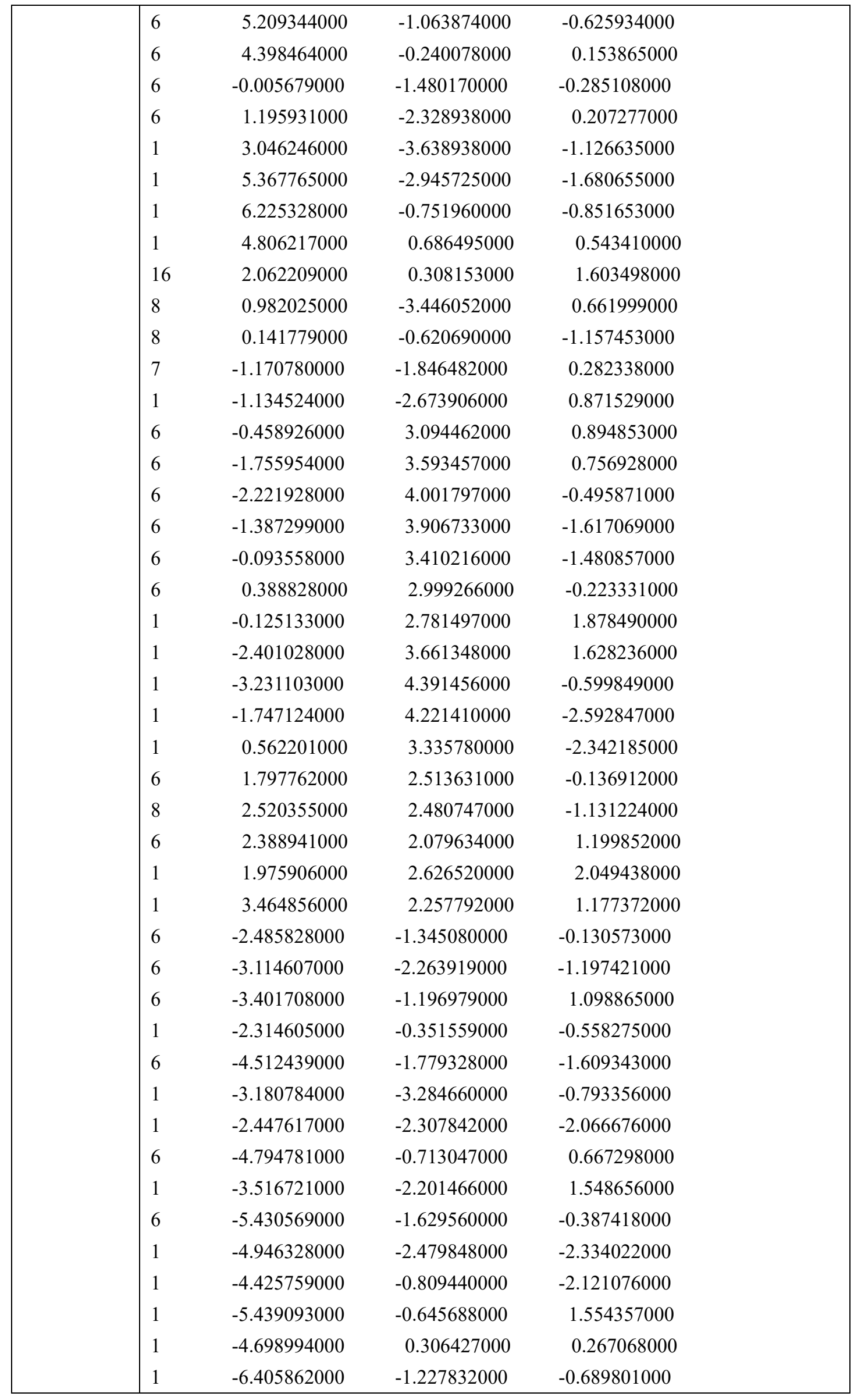




\begin{tabular}{|c|c|c|c|c|}
\hline & 1 & -5.620053000 & -2.620585000 & 0.050937000 \\
\hline & 7 & -2.817105000 & -0.238230000 & 2.051006000 \\
\hline & 1 & -3.390501000 & -0.203730000 & 2.893923000 \\
\hline & 1 & -1.896104000 & -0.559808000 & 2.349148000 \\
\hline \multirow{40}{*}{ TS-4 } & 6 & -0.178828000 & 2.269292000 & -1.490508000 \\
\hline & 6 & -1.341639000 & 2.202334000 & -2.298196000 \\
\hline & 6 & -1.704264000 & 3.303711000 & -3.092189000 \\
\hline & 6 & -0.970123000 & 4.487760000 & -3.058387000 \\
\hline & 6 & 0.160345000 & 4.562064000 & -2.238807000 \\
\hline & 6 & 0.558142000 & 3.462919000 & -1.474814000 \\
\hline & 6 & -2.832342000 & 0.415606000 & -1.097101000 \\
\hline & 6 & -2.215988000 & 0.998508000 & -2.392199000 \\
\hline & 1 & -2.589492000 & 3.225696000 & -3.717336000 \\
\hline & 1 & -1.275892000 & 5.338540000 & -3.660135000 \\
\hline & 1 & 0.746514000 & 5.476621000 & -2.198354000 \\
\hline & 1 & 1.456258000 & 3.530099000 & -0.870648000 \\
\hline & 16 & 0.373780000 & 0.796249000 & -0.627569000 \\
\hline & 8 & -2.571845000 & 0.535398000 & -3.470323000 \\
\hline & 8 & -3.084090000 & 1.159603000 & -0.140952000 \\
\hline & 7 & -3.122906000 & -0.891819000 & -1.167479000 \\
\hline & 1 & -2.877071000 & -1.381678000 & -2.020381000 \\
\hline & 6 & 0.165457000 & 0.841042000 & 2.750076000 \\
\hline & 6 & -0.794775000 & 0.849981000 & 3.765874000 \\
\hline & 6 & -1.086117000 & 2.035700000 & 4.448026000 \\
\hline & 6 & -0.399517000 & 3.210701000 & 4.120870000 \\
\hline & 6 & 0.576730000 & 3.194486000 & 3.122839000 \\
\hline & 6 & 0.863966000 & 2.013282000 & 2.418163000 \\
\hline & 1 & 0.380889000 & -0.086851000 & 2.230401000 \\
\hline & 1 & -1.315606000 & -0.068885000 & 4.022874000 \\
\hline & 1 & -1.840097000 & 2.043534000 & 5.231031000 \\
\hline & 1 & -0.621038000 & 4.136237000 & 4.646248000 \\
\hline & 1 & 1.123012000 & 4.100469000 & 2.876799000 \\
\hline & 6 & 1.961900000 & 2.043292000 & 1.385962000 \\
\hline & 8 & 2.972758000 & 2.739193000 & 1.631073000 \\
\hline & 6 & 1.911447000 & 1.218145000 & 0.188373000 \\
\hline & 1 & 2.474755000 & -0.106249000 & 0.578395000 \\
\hline & 1 & 2.686110000 & 1.511712000 & -0.525639000 \\
\hline & 7 & 3.018644000 & -1.218719000 & 0.794397000 \\
\hline & 1 & 2.318092000 & -1.959819000 & 0.667896000 \\
\hline & 1 & 3.330211000 & -1.256628000 & 1.767935000 \\
\hline & 6 & 4.140712000 & -1.498180000 & -0.145909000 \\
\hline & 6 & 5.406735000 & -0.739794000 & 0.264954000 \\
\hline & 6 & 4.378723000 & -3.017712000 & -0.253602000 \\
\hline & 1 & 3.801696000 & -1.132169000 & -1.125377000 \\
\hline
\end{tabular}




\begin{tabular}{|c|c|c|c|c|}
\hline & 6 & 6.565189000 & -1.039878000 & -0.699923000 \\
\hline & 1 & 5.690495000 & -1.043528000 & 1.284005000 \\
\hline & 1 & 5.201437000 & 0.337078000 & 0.297426000 \\
\hline & 6 & 5.542741000 & -3.304676000 & -1.219557000 \\
\hline & 1 & 4.654935000 & -3.383277000 & 0.745961000 \\
\hline & 6 & 6.821326000 & -2.550266000 & -0.819629000 \\
\hline & 1 & 7.470261000 & -0.524564000 & -0.356113000 \\
\hline & 1 & 6.326589000 & -0.629487000 & -1.691865000 \\
\hline & 1 & 5.725125000 & -4.386607000 & -1.247999000 \\
\hline & 1 & 5.240520000 & -3.006571000 & -2.235344000 \\
\hline & 1 & 7.613779000 & -2.742891000 & -1.553656000 \\
\hline & 1 & 7.185447000 & -2.935527000 & 0.144290000 \\
\hline & 7 & 3.103527000 & -3.670778000 & -0.597751000 \\
\hline & 1 & 2.905697000 & -3.557741000 & -1.592881000 \\
\hline & 1 & 3.164279000 & -4.673849000 & -0.430998000 \\
\hline & 6 & -3.783918000 & -1.643046000 & -0.097707000 \\
\hline & 6 & -5.305778000 & -1.388524000 & -0.098771000 \\
\hline & 6 & -3.470474000 & -3.145849000 & -0.231522000 \\
\hline & 1 & -3.366364000 & -1.277075000 & 0.850384000 \\
\hline & 6 & -6.013905000 & -2.177815000 & 1.011395000 \\
\hline & 1 & -5.707397000 & -1.684700000 & -1.078553000 \\
\hline & 1 & -5.487698000 & -0.314089000 & 0.013021000 \\
\hline & 6 & -4.193703000 & -3.931746000 & 0.881902000 \\
\hline & 1 & -3.864523000 & -3.484839000 & -1.201754000 \\
\hline & 6 & -5.709165000 & -3.677938000 & 0.901278000 \\
\hline & 1 & -7.095393000 & -2.000112000 & 0.957447000 \\
\hline & 1 & -5.682689000 & -1.806741000 & 1.992701000 \\
\hline & 1 & -3.985265000 & -5.001424000 & 0.748304000 \\
\hline & 1 & -3.759015000 & -3.642867000 & 1.851204000 \\
\hline & 1 & -6.169256000 & -4.226086000 & 1.733311000 \\
\hline & 1 & -6.157103000 & -4.073357000 & -0.022510000 \\
\hline & 7 & -2.014924000 & -3.362207000 & -0.276997000 \\
\hline & 1 & -1.601445000 & -3.128770000 & 0.627196000 \\
\hline & 1 & -1.823490000 & -4.352879000 & -0.425111000 \\
\hline \multirow{10}{*}{ INT-4 } & 6 & -1.836600000 & 1.818405000 & -0.831442000 \\
\hline & 6 & -1.051095000 & 1.848397000 & 0.334592000 \\
\hline & 6 & -1.185655000 & 2.935935000 & 1.215304000 \\
\hline & 6 & -2.048239000 & 3.997204000 & 0.935547000 \\
\hline & 6 & -2.800695000 & 3.974573000 & -0.243627000 \\
\hline & 6 & -2.699676000 & 2.889064000 & -1.114583000 \\
\hline & 6 & 1.310144000 & 0.846952000 & -0.081048000 \\
\hline & 6 & -0.015383000 & 0.824247000 & 0.718800000 \\
\hline & 1 & -0.585640000 & 2.950227000 & 2.121923000 \\
\hline & 1 & -2.130407000 & 4.829853000 & 1.628718000 \\
\hline
\end{tabular}




\begin{tabular}{|c|c|c|c|c|}
\hline & 1 & -3.481268000 & 4.789737000 & -0.476698000 \\
\hline & 1 & -3.313340000 & 2.856786000 & -2.012252000 \\
\hline & 16 & -1.846852000 & 0.416945000 & -1.949852000 \\
\hline & 8 & -0.021042000 & 0.283943000 & 1.832196000 \\
\hline & 8 & 1.486987000 & 1.613639000 & -1.040508000 \\
\hline & 7 & 2.243372000 & 0.018274000 & 0.419948000 \\
\hline & 1 & 1.967362000 & -0.522101000 & 1.233531000 \\
\hline & 6 & -3.879414000 & -2.134759000 & -0.840109000 \\
\hline & 6 & -5.221611000 & -2.251463000 & -0.464376000 \\
\hline & 6 & -5.601694000 & -2.027342000 & 0.863519000 \\
\hline & 6 & -4.628548000 & -1.698620000 & 1.814101000 \\
\hline & 6 & -3.284782000 & -1.602236000 & 1.439426000 \\
\hline & 6 & -2.896307000 & -1.808124000 & 0.106427000 \\
\hline & 1 & -3.592056000 & -2.304553000 & -1.874811000 \\
\hline & 1 & -5.969695000 & -2.514615000 & -1.208592000 \\
\hline & 1 & -6.645734000 & -2.110687000 & 1.155341000 \\
\hline & 1 & -4.915016000 & -1.523039000 & 2.848480000 \\
\hline & 1 & -2.529307000 & -1.360235000 & 2.182419000 \\
\hline & 6 & -1.425271000 & -1.792730000 & -0.258381000 \\
\hline & 8 & -0.705466000 & -2.686579000 & 0.282878000 \\
\hline & 6 & -0.889815000 & -0.800639000 & -1.106209000 \\
\hline & 1 & 0.126790000 & -0.943244000 & -1.457890000 \\
\hline & 6 & 3.621043000 & -0.059849000 & -0.060779000 \\
\hline & 6 & 4.512823000 & 0.948192000 & 0.696921000 \\
\hline & 6 & 4.171974000 & -1.495869000 & 0.056894000 \\
\hline & 1 & 3.596304000 & 0.219813000 & -1.121779000 \\
\hline & 6 & 5.977023000 & 0.890283000 & 0.241214000 \\
\hline & 1 & 4.452442000 & 0.725212000 & 1.772446000 \\
\hline & 1 & 4.102460000 & 1.955292000 & 0.557041000 \\
\hline & 6 & 5.650263000 & -1.534469000 & -0.387483000 \\
\hline & 1 & 4.124780000 & -1.789195000 & 1.116755000 \\
\hline & 6 & 6.534219000 & -0.533514000 & 0.370818000 \\
\hline & 1 & 6.576625000 & 1.594986000 & 0.831574000 \\
\hline & 1 & 6.048440000 & 1.216809000 & -0.807025000 \\
\hline & 1 & 6.027855000 & -2.557270000 & -0.255678000 \\
\hline & 1 & 5.694333000 & -1.318940000 & -1.466537000 \\
\hline & 1 & 7.563565000 & -0.580831000 & -0.007345000 \\
\hline & 1 & 6.575645000 & -0.813841000 & 1.434040000 \\
\hline & 7 & 3.309500000 & -2.437399000 & -0.675624000 \\
\hline & 1 & 3.357721000 & -2.241957000 & -1.676808000 \\
\hline & 1 & 3.667596000 & -3.385300000 & -0.559180000 \\
\hline & 6 & -1.911107000 & 1.860491000 & -0.842289000 \\
\hline TS-5 & 6 & -1.050281000 & 1.796800000 & 0.260225000 \\
\hline & 6 & -1.043344000 & 2.862050000 & 1.170893000 \\
\hline
\end{tabular}




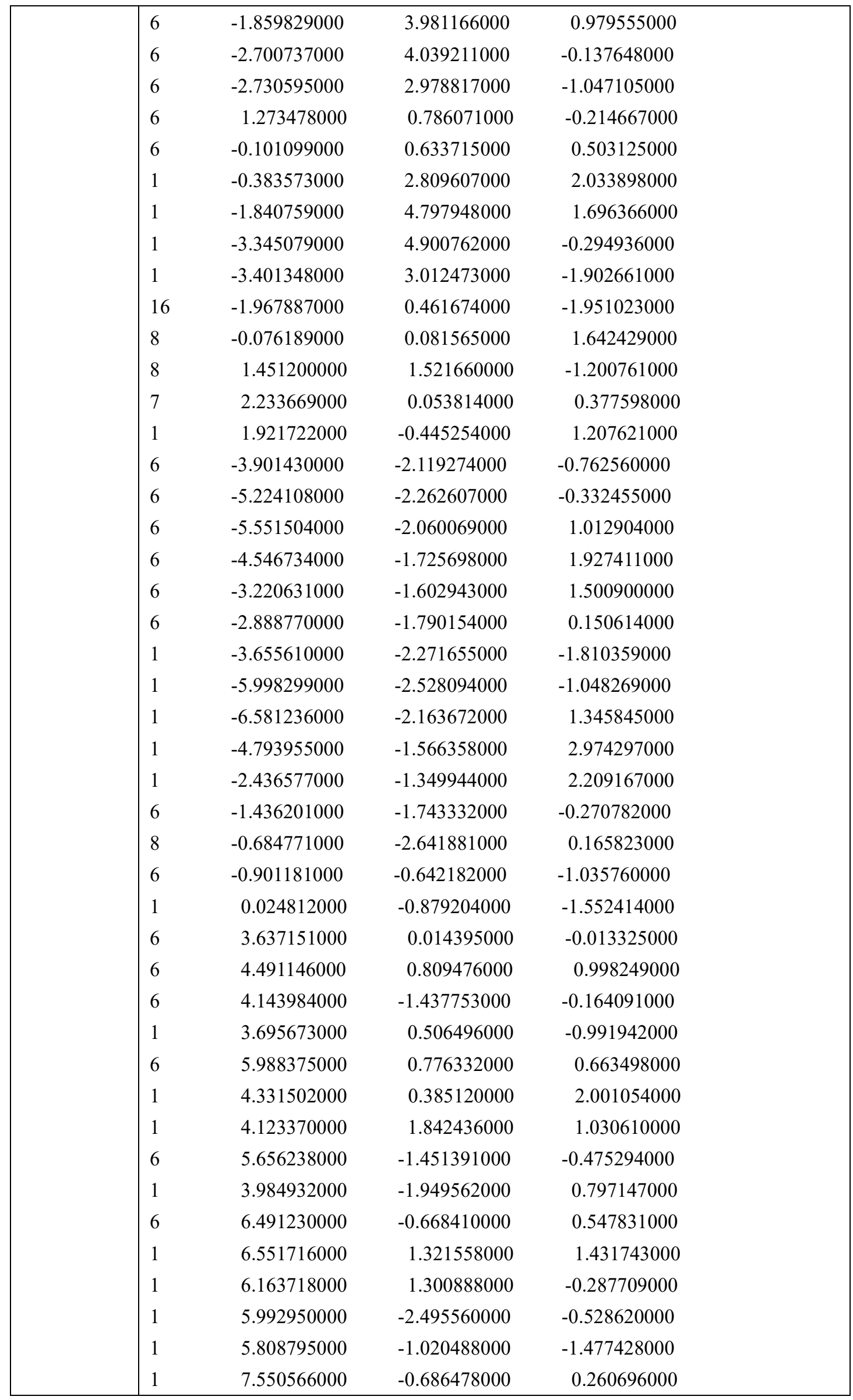




\begin{tabular}{|c|c|c|c|c|}
\hline & 1 & 6.422240000 & -1.158703000 & 1.530555000 \\
\hline & 7 & 3.336324000 & -2.160495000 & -1.161397000 \\
\hline & 1 & 3.508128000 & -1.770148000 & -2.089424000 \\
\hline & 1 & 3.646330000 & -3.131205000 & -1.207585000 \\
\hline \multirow{40}{*}{ INT-5 } & 6 & 1.795815000 & 2.066055000 & 0.842270000 \\
\hline & 6 & 0.802134000 & 1.724369000 & -0.079191000 \\
\hline & 6 & 0.396179000 & 2.674105000 & -1.019035000 \\
\hline & 6 & 0.970859000 & 3.950653000 & -1.029583000 \\
\hline & 6 & 1.965062000 & 4.277788000 & -0.097764000 \\
\hline & 6 & 2.389342000 & 3.333766000 & 0.843637000 \\
\hline & 6 & -1.232237000 & 0.426073000 & 0.593442000 \\
\hline & 6 & 0.226257000 & 0.292526000 & -0.010383000 \\
\hline & 1 & -0.367005000 & 2.407990000 & -1.747240000 \\
\hline & 1 & 0.648586000 & 4.686469000 & -1.762113000 \\
\hline & 1 & 2.418665000 & 5.265890000 & -0.107864000 \\
\hline & 1 & 3.170247000 & 3.581921000 & 1.558401000 \\
\hline & 16 & 2.222288000 & 0.752821000 & 1.967921000 \\
\hline & 8 & 0.226833000 & -0.365762000 & -1.187803000 \\
\hline & 8 & -1.473228000 & 0.940705000 & 1.703209000 \\
\hline & 7 & -2.151312000 & -0.064846000 & -0.247685000 \\
\hline & 1 & -1.711991000 & -0.465540000 & -1.083289000 \\
\hline & 6 & 3.495067000 & -0.566606000 & -0.926998000 \\
\hline & 6 & 4.696012000 & -0.611336000 & -1.637651000 \\
\hline & 6 & 5.507900000 & -1.749833000 & -1.581185000 \\
\hline & 6 & 5.107618000 & -2.851753000 & -0.817201000 \\
\hline & 6 & 3.899312000 & -2.813079000 & -0.120128000 \\
\hline & 6 & 3.081152000 & -1.670133000 & -0.163102000 \\
\hline & 1 & 2.864792000 & 0.311256000 & -0.994510000 \\
\hline & 1 & 4.998375000 & 0.243604000 & -2.236897000 \\
\hline & 1 & 6.445943000 & -1.778148000 & -2.129820000 \\
\hline & 1 & 5.734047000 & -3.738533000 & -0.766585000 \\
\hline & 1 & 3.580525000 & -3.666015000 & 0.471204000 \\
\hline & 6 & 1.791962000 & -1.707065000 & 0.600295000 \\
\hline & 8 & 1.277605000 & -2.794460000 & 0.863501000 \\
\hline & 6 & 1.093799000 & -0.456443000 & 1.140780000 \\
\hline & 1 & 0.419699000 & -0.824965000 & 1.914608000 \\
\hline & 6 & -3.590944000 & -0.079791000 & -0.047130000 \\
\hline & 6 & -4.284334000 & 0.700382000 & -1.183627000 \\
\hline & 6 & -4.146929000 & -1.518955000 & 0.067654000 \\
\hline & 1 & -3.773684000 & 0.436572000 & 0.904274000 \\
\hline & 6 & -5.813267000 & 0.705408000 & -1.050084000 \\
\hline & 1 & -4.003487000 & 0.244181000 & -2.145202000 \\
\hline & 1 & -3.893205000 & 1.725223000 & -1.194765000 \\
\hline & 6 & -5.686311000 & -1.493704000 & 0.184136000 \\
\hline
\end{tabular}




\begin{tabular}{|c|c|c|c|c|}
\hline & 1 & -3.878198000 & -2.054990000 & -0.855110000 \\
\hline & 6 & -6.362787000 & -0.724252000 & -0.959341000 \\
\hline & 1 & -6.257944000 & 1.236537000 & -1.901636000 \\
\hline & 1 & -6.099608000 & 1.263755000 & -0.146192000 \\
\hline & 1 & -6.053788000 & -2.528245000 & 0.221982000 \\
\hline & 1 & -5.954905000 & -1.029394000 & 1.146221000 \\
\hline & 1 & -7.450446000 & -0.709747000 & -0.811217000 \\
\hline & 1 & -6.181544000 & -1.246759000 & -1.910726000 \\
\hline & 7 & -3.488617000 & -2.234879000 & 1.174138000 \\
\hline & 1 & -3.753710000 & -1.808424000 & 2.063430000 \\
\hline & 1 & -3.838111000 & -3.192599000 & 1.211034000 \\
\hline \multirow{33}{*}{ INT-5-1 } & 6 & 1.753556000 & 2.170692000 & 0.816115000 \\
\hline & 6 & 0.779332000 & 1.741785000 & -0.093686000 \\
\hline & 6 & 0.322269000 & 2.612048000 & -1.087392000 \\
\hline & 6 & 0.846495000 & 3.905449000 & -1.169775000 \\
\hline & 6 & 1.819607000 & 4.325943000 & -0.254198000 \\
\hline & 6 & 2.282847000 & 3.463481000 & 0.743769000 \\
\hline & 6 & -1.206660000 & 0.367924000 & 0.584843000 \\
\hline & 6 & 0.276559000 & 0.319575000 & 0.103572000 \\
\hline & 1 & -0.437821000 & 2.283179000 & -1.791722000 \\
\hline & 1 & 0.495512000 & 4.583350000 & -1.942258000 \\
\hline & 1 & 2.226309000 & 5.331506000 & -0.319262000 \\
\hline & 1 & 3.044034000 & 3.791745000 & 1.445747000 \\
\hline & 16 & 2.230416000 & 0.946085000 & 2.008427000 \\
\hline & 8 & 0.312232000 & -0.446270000 & -1.103024000 \\
\hline & 8 & -1.462568000 & 0.816204000 & 1.709719000 \\
\hline & 7 & -2.113963000 & -0.057368000 & -0.306697000 \\
\hline & 1 & -1.750649000 & -0.443024000 & -1.171990000 \\
\hline & 6 & 3.590223000 & -0.537621000 & -0.733599000 \\
\hline & 6 & 4.703693000 & -0.650627000 & -1.568060000 \\
\hline & 6 & 5.335350000 & -1.886893000 & -1.733514000 \\
\hline & 6 & 4.844576000 & -3.014219000 & -1.064096000 \\
\hline & 6 & 3.727145000 & -2.903387000 & -0.239355000 \\
\hline & 6 & 3.082155000 & -1.664163000 & -0.062287000 \\
\hline & 1 & 3.135175000 & 0.438228000 & -0.611959000 \\
\hline & 1 & 5.079594000 & 0.228909000 & -2.083035000 \\
\hline & 1 & 6.205569000 & -1.971484000 & -2.378832000 \\
\hline & 1 & 5.331898000 & -3.977414000 & -1.187540000 \\
\hline & 1 & 3.338224000 & -3.773963000 & 0.278188000 \\
\hline & 6 & 1.875268000 & -1.638274000 & 0.820713000 \\
\hline & 8 & 1.416888000 & -2.686230000 & 1.268224000 \\
\hline & 6 & 1.140919000 & -0.347997000 & 1.247594000 \\
\hline & 1 & 0.470519000 & -0.672755000 & 2.041716000 \\
\hline & 1 & 1.025532000 & -0.124934000 & -1.679344000 \\
\hline
\end{tabular}




\begin{tabular}{|c|c|c|c|c|}
\hline & 6 & -3.557284000 & -0.093125000 & -0.072796000 \\
\hline & 6 & -4.282240000 & 0.693386000 & -1.181932000 \\
\hline & 6 & -4.080676000 & -1.544979000 & 0.029790000 \\
\hline & 1 & -3.719842000 & 0.405076000 & 0.889429000 \\
\hline & 6 & -5.807046000 & 0.672571000 & -1.004706000 \\
\hline & 1 & -4.022036000 & 0.253887000 & -2.156875000 \\
\hline & 1 & -3.907123000 & 1.723965000 & -1.188148000 \\
\hline & 6 & -5.615537000 & -1.544139000 & 0.193672000 \\
\hline & 1 & -3.834151000 & -2.056800000 & -0.912486000 \\
\hline & 6 & -6.334847000 & -0.765791000 & -0.917497000 \\
\hline & 1 & -6.281644000 & 1.207959000 & -1.836746000 \\
\hline & 1 & -6.074816000 & 1.215915000 & -0.086440000 \\
\hline & 1 & -5.965482000 & -2.584641000 & 0.223615000 \\
\hline & 1 & -5.861865000 & -1.100702000 & 1.171179000 \\
\hline & 1 & -7.417243000 & -0.766366000 & -0.735925000 \\
\hline & 1 & -6.176973000 & -1.272659000 & -1.881272000 \\
\hline & 7 & -3.370002000 & -2.269028000 & 1.096245000 \\
\hline & 1 & -3.633908000 & -1.888532000 & 2.006384000 \\
\hline & 1 & -3.674341000 & -3.242478000 & 1.103512000 \\
\hline \multirow{25}{*}{ TS-6 } & 6 & -0.527581000 & 3.165421000 & -0.235791000 \\
\hline & 6 & 0.726776000 & 2.548930000 & -0.371737000 \\
\hline & 6 & 1.701871000 & 3.138053000 & -1.179705000 \\
\hline & 6 & 1.421024000 & 4.332874000 & -1.850191000 \\
\hline & 6 & 0.166697000 & 4.941900000 & -1.704662000 \\
\hline & 6 & -0.817294000 & 4.364494000 & -0.897140000 \\
\hline & 6 & 1.814272000 & 0.264184000 & -0.163641000 \\
\hline & 6 & 0.901250000 & 1.317870000 & 0.512428000 \\
\hline & 1 & 2.677440000 & 2.670210000 & -1.286491000 \\
\hline & 1 & 2.176113000 & 4.788018000 & -2.485123000 \\
\hline & 1 & -0.049429000 & 5.869351000 & -2.228730000 \\
\hline & 1 & -1.790455000 & 4.836194000 & -0.791157000 \\
\hline & 16 & -1.621257000 & 2.296906000 & 0.856870000 \\
\hline & 8 & 1.571230000 & 1.721691000 & 1.741937000 \\
\hline & 8 & 1.473181000 & -0.268270000 & -1.231344000 \\
\hline & 7 & 2.999367000 & 0.046976000 & 0.432540000 \\
\hline & 1 & 3.098987000 & 0.393789000 & 1.380371000 \\
\hline & 6 & -2.512681000 & 0.443054000 & 3.312383000 \\
\hline & 6 & -3.652172000 & 0.167499000 & 4.076206000 \\
\hline & 6 & -4.293116000 & -1.069054000 & 3.962088000 \\
\hline & 6 & -3.777131000 & -2.040682000 & 3.095697000 \\
\hline & 6 & -2.625394000 & -1.774844000 & 2.353938000 \\
\hline & 6 & -1.989673000 & -0.524083000 & 2.440143000 \\
\hline & 1 & -2.018730000 & 1.403970000 & 3.420497000 \\
\hline & 1 & -4.036079000 & 0.920300000 & 4.759892000 \\
\hline
\end{tabular}




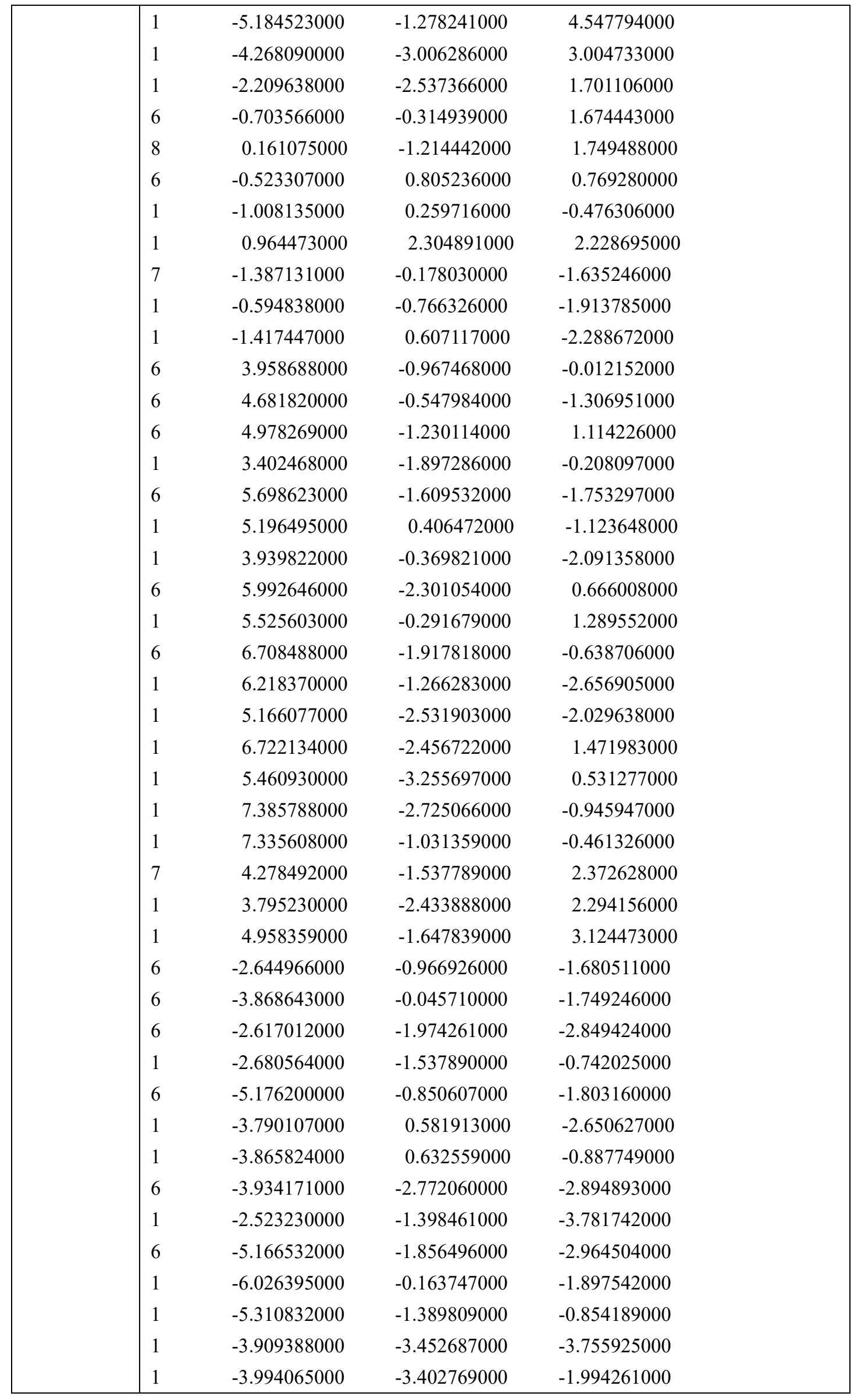




\begin{tabular}{|c|c|c|c|c|}
\hline & 1 & -6.082971000 & -2.460068000 & -2.953442000 \\
\hline & 1 & -5.161040000 & -1.310183000 & -3.919412000 \\
\hline & 7 & -1.400282000 & -2.797841000 & -2.744733000 \\
\hline & 1 & -1.513753000 & -3.500953000 & -2.012984000 \\
\hline & 1 & -1.251180000 & -3.313016000 & -3.611108000 \\
\hline & 6 & -1.374223000 & 2.741215000 & -0.354574000 \\
\hline & 6 & -0.252002000 & 2.310784000 & 0.368389000 \\
\hline & 6 & 0.666781000 & 3.248231000 & 0.842491000 \\
\hline & 6 & 0.466840000 & 4.610989000 & 0.591886000 \\
\hline & 6 & -0.650566000 & 5.030177000 & -0.143036000 \\
\hline & 6 & -1.578859000 & 4.099918000 & -0.621431000 \\
\hline & 6 & 1.037539000 & 0.292716000 & -0.349733000 \\
\hline & 6 & -0.145642000 & 0.802333000 & 0.547154000 \\
\hline & 1 & 1.534330000 & 2.916983000 & 1.408100000 \\
\hline & 1 & 1.179324000 & 5.342095000 & 0.964396000 \\
\hline & 1 & -0.805640000 & 6.088300000 & -0.338925000 \\
\hline & 1 & -2.449647000 & 4.430740000 & -1.181577000 \\
\hline & 16 & -2.438330000 & 1.417034000 & -0.864545000 \\
\hline & 8 & 0.116692000 & 0.484956000 & 1.921622000 \\
\hline & 8 & 0.990514000 & 0.385876000 & -1.587344000 \\
\hline & 7 & 2.091454000 & -0.193671000 & 0.331514000 \\
\hline & 1 & 1.982401000 & -0.209896000 & 1.341050000 \\
\hline & 6 & -4.085998000 & -2.134829000 & 1.208566000 \\
\hline & 6 & -5.337579000 & -2.670621000 & 0.899516000 \\
\hline INT-6 & 6 & -5.815201000 & -2.631300000 & -0.416864000 \\
\hline & 6 & -5.027417000 & -2.052697000 & -1.416382000 \\
\hline & 6 & -3.777883000 & -1.507015000 & -1.101097000 \\
\hline & 6 & -3.291351000 & -1.530113000 & 0.217135000 \\
\hline & 1 & -3.709725000 & -2.175583000 & 2.226643000 \\
\hline & 1 & -5.941472000 & -3.120869000 & 1.684061000 \\
\hline & 1 & -6.786868000 & -3.053864000 & -0.660448000 \\
\hline & 1 & -5.379153000 & -2.033486000 & -2.445261000 \\
\hline & 1 & -3.166081000 & -1.085658000 & -1.894436000 \\
\hline & 6 & -1.950006000 & -0.974188000 & 0.598253000 \\
\hline & 8 & -1.251292000 & -1.669768000 & 1.466945000 \\
\hline & 6 & -1.484582000 & 0.220282000 & 0.093079000 \\
\hline & 1 & -0.295584000 & -0.431746000 & 2.011423000 \\
\hline & 6 & 3.338451000 & -0.661114000 & -0.265821000 \\
\hline & 6 & 4.503446000 & 0.248141000 & 0.176729000 \\
\hline & 6 & 3.619134000 & -2.143450000 & 0.071200000 \\
\hline & 1 & 3.206027000 & -0.571432000 & -1.350426000 \\
\hline & 6 & 5.852287000 & -0.202366000 & -0.400853000 \\
\hline & 1 & 4.556987000 & 0.242523000 & 1.276082000 \\
\hline & 1 & 4.278342000 & 1.278856000 & -0.123667000 \\
\hline
\end{tabular}




\begin{tabular}{|c|c|c|c|c|}
\hline & 6 & 4.987707000 & -2.572251000 & -0.501173000 \\
\hline & 1 & 3.658878000 & -2.235920000 & 1.167024000 \\
\hline & 6 & 6.141001000 & -1.666897000 & -0.045753000 \\
\hline & 1 & 6.651592000 & 0.450665000 & -0.027486000 \\
\hline & 1 & 5.837561000 & -0.088916000 & -1.495116000 \\
\hline & 1 & 5.178467000 & -3.614353000 & -0.211064000 \\
\hline & 1 & 4.924416000 & -2.558157000 & -1.600730000 \\
\hline & 1 & 7.081545000 & -1.996326000 & -0.506007000 \\
\hline & 1 & 6.273278000 & -1.759806000 & 1.042686000 \\
\hline & 7 & 2.502911000 & -2.990600000 & -0.380687000 \\
\hline & 1 & 2.467105000 & -2.996323000 & -1.401284000 \\
\hline & 1 & 2.678068000 & -3.956652000 & -0.103978000 \\
\hline \multirow{32}{*}{ INT-6-1 } & 6 & -1.334047000 & 2.836591000 & -0.296836000 \\
\hline & 6 & -0.184658000 & 2.375745000 & 0.355679000 \\
\hline & 6 & 0.817217000 & 3.275575000 & 0.725281000 \\
\hline & 6 & 0.661029000 & 4.635752000 & 0.441063000 \\
\hline & 6 & -0.490216000 & 5.086662000 & -0.218703000 \\
\hline & 6 & -1.497747000 & 4.192759000 & -0.593665000 \\
\hline & 6 & 0.982110000 & 0.250386000 & -0.297178000 \\
\hline & 6 & -0.146578000 & 0.875066000 & 0.585990000 \\
\hline & 1 & 1.710161000 & 2.918998000 & 1.232137000 \\
\hline & 1 & 1.434682000 & 5.341365000 & 0.729492000 \\
\hline & 1 & -0.608267000 & 6.144113000 & -0.439589000 \\
\hline & 1 & -2.390987000 & 4.549047000 & -1.098823000 \\
\hline & 16 & -2.497567000 & 1.555641000 & -0.689986000 \\
\hline & 8 & 0.172258000 & 0.546417000 & 1.961251000 \\
\hline & 8 & 0.833304000 & 0.207473000 & -1.523707000 \\
\hline & 7 & 2.083785000 & -0.149878000 & 0.361012000 \\
\hline & 1 & 2.069646000 & -0.076672000 & 1.372186000 \\
\hline & 6 & -3.942583000 & -2.304949000 & 1.116357000 \\
\hline & 6 & -5.164908000 & -2.895203000 & 0.793752000 \\
\hline & 6 & -5.720055000 & -2.716482000 & -0.478874000 \\
\hline & 6 & -5.039494000 & -1.948131000 & -1.428074000 \\
\hline & 6 & -3.817070000 & -1.352829000 & -1.106454000 \\
\hline & 6 & -3.258717000 & -1.512688000 & 0.173990000 \\
\hline & 1 & -3.512890000 & -2.448243000 & 2.102641000 \\
\hline & 1 & -5.684829000 & -3.495518000 & 1.535790000 \\
\hline & 1 & -6.670679000 & -3.179243000 & -0.730368000 \\
\hline & 1 & -5.452473000 & -1.820218000 & -2.425145000 \\
\hline & 1 & -3.282898000 & -0.790716000 & -1.866553000 \\
\hline & 6 & -1.966470000 & -0.896621000 & 0.544633000 \\
\hline & 8 & -1.210044000 & -1.715125000 & 1.349358000 \\
\hline & 6 & -1.521944000 & 0.333032000 & 0.185704000 \\
\hline & 1 & -0.543407000 & -1.163832000 & 1.813097000 \\
\hline
\end{tabular}




\begin{tabular}{|c|c|c|c|c|}
\hline & 1 & -0.381268000 & 1.089025000 & 2.550329000 \\
\hline & 6 & 3.285951000 & -0.688018000 & -0.278156000 \\
\hline & 6 & 4.501107000 & 0.184051000 & 0.094968000 \\
\hline & 6 & 3.516695000 & -2.171647000 & 0.090589000 \\
\hline & 1 & 3.109505000 & -0.621023000 & -1.357294000 \\
\hline & 6 & 5.805410000 & -0.338700000 & -0.523293000 \\
\hline & 1 & 4.599915000 & 0.202937000 & 1.190953000 \\
\hline & 1 & 4.307285000 & 1.215332000 & -0.224005000 \\
\hline & 6 & 4.839550000 & -2.671829000 & -0.528398000 \\
\hline & 1 & 3.601080000 & -2.235207000 & 1.185783000 \\
\hline & 6 & 6.047224000 & -1.805575000 & -0.143378000 \\
\hline & 1 & 6.644461000 & 0.288575000 & -0.196543000 \\
\hline & 1 & 5.752454000 & -0.249119000 & -1.618399000 \\
\hline & 1 & 4.998480000 & -3.713746000 & -0.219855000 \\
\hline & 1 & 4.729712000 & -2.680800000 & -1.624183000 \\
\hline & 1 & 6.953032000 & -2.184816000 & -0.633515000 \\
\hline & 1 & 6.221082000 & -1.878936000 & 0.940643000 \\
\hline & 7 & 2.345733000 & -2.979766000 & -0.286511000 \\
\hline & 1 & 2.267621000 & -3.019145000 & -1.303954000 \\
\hline & 1 & 2.488042000 & -3.942702000 & 0.018243000 \\
\hline \multirow{24}{*}{ TS-7 } & 6 & -1.428014000 & 2.917499000 & 0.022688000 \\
\hline & 6 & -0.163424000 & 2.303032000 & 0.179154000 \\
\hline & 6 & 0.988116000 & 3.106571000 & 0.287948000 \\
\hline & 6 & 0.863712000 & 4.489814000 & 0.259222000 \\
\hline & 6 & -0.403884000 & 5.086775000 & 0.111186000 \\
\hline & 6 & -1.554922000 & 4.312136000 & -0.012860000 \\
\hline & 6 & 0.907580000 & 0.089165000 & -0.504727000 \\
\hline & 6 & -0.232081000 & 0.846310000 & 0.198423000 \\
\hline & 1 & 1.963026000 & 2.642804000 & 0.402785000 \\
\hline & 1 & 1.746785000 & 5.115216000 & 0.350125000 \\
\hline & 1 & -0.487625000 & 6.169868000 & 0.088865000 \\
\hline & 1 & -2.527006000 & 4.780310000 & -0.135891000 \\
\hline & 16 & -2.739791000 & 1.763557000 & -0.148610000 \\
\hline & 8 & 0.230168000 & 0.179910000 & 2.055838000 \\
\hline & 8 & 0.763206000 & -0.127923000 & -1.717389000 \\
\hline & 7 & 2.013279000 & -0.177781000 & 0.201218000 \\
\hline & 1 & 1.937822000 & -0.030558000 & 1.204984000 \\
\hline & 6 & -3.769568000 & -2.540910000 & 0.847289000 \\
\hline & 6 & -4.988560000 & -3.147500000 & 0.549778000 \\
\hline & 6 & -5.706664000 & -2.759855000 & -0.588063000 \\
\hline & 6 & -5.198834000 & -1.764599000 & -1.430512000 \\
\hline & 6 & -3.982595000 & -1.149622000 & -1.133379000 \\
\hline & 6 & -3.258611000 & -1.526598000 & 0.013997000 \\
\hline & 1 & -3.210168000 & -2.835689000 & 1.729026000 \\
\hline
\end{tabular}




\begin{tabular}{|c|c|c|c|c|}
\hline & 1 & -5.380444000 & -3.921230000 & 1.204059000 \\
\hline & 1 & -6.655350000 & -3.236122000 & -0.820384000 \\
\hline & 1 & -5.745063000 & -1.474279000 & -2.323517000 \\
\hline & 1 & -3.579405000 & -0.401097000 & -1.808481000 \\
\hline & 6 & -1.968124000 & -0.904107000 & 0.341748000 \\
\hline & 8 & -1.118654000 & -1.634934000 & 1.040016000 \\
\hline & 6 & -1.590488000 & 0.403907000 & 0.037312000 \\
\hline & 1 & -0.472074000 & -0.943809000 & 1.583081000 \\
\hline & 1 & -0.348122000 & 0.723681000 & 2.617634000 \\
\hline & 6 & 3.225679000 & -0.774251000 & -0.360282000 \\
\hline & 6 & 4.337847000 & 0.290555000 & -0.459536000 \\
\hline & 6 & 3.681005000 & -1.996586000 & 0.466841000 \\
\hline & 1 & 2.964743000 & -1.112248000 & -1.369582000 \\
\hline & 6 & 5.647388000 & -0.285706000 & -1.014640000 \\
\hline & 1 & 4.515540000 & 0.708343000 & 0.542516000 \\
\hline & 1 & 3.980518000 & 1.115047000 & -1.088416000 \\
\hline & 6 & 5.007639000 & -2.551116000 & -0.094919000 \\
\hline & 1 & 3.859049000 & -1.656780000 & 1.498253000 \\
\hline & 6 & 6.113151000 & -1.489048000 & -0.183926000 \\
\hline & 1 & 6.418130000 & 0.495323000 & -1.028703000 \\
\hline & 1 & 5.496250000 & -0.598822000 & -2.058394000 \\
\hline & 1 & 5.330784000 & -3.390471000 & 0.535282000 \\
\hline & 1 & 4.815121000 & -2.965711000 & -1.096815000 \\
\hline & 1 & 7.020345000 & -1.928355000 & -0.618326000 \\
\hline & 1 & 6.380200000 & -1.152114000 & 0.828852000 \\
\hline & 7 & 2.602514000 & -2.996313000 & 0.536539000 \\
\hline & 1 & 2.453018000 & -3.407116000 & -0.386418000 \\
\hline & 1 & 2.894179000 & -3.766076000 & 1.138758000 \\
\hline \multirow{16}{*}{ INT-7 } & 6 & -1.771238000 & 2.860931000 & 0.272780000 \\
\hline & 6 & -0.513275000 & 2.433090000 & -0.219072000 \\
\hline & 6 & 0.413762000 & 3.402104000 & -0.658120000 \\
\hline & 6 & 0.079187000 & 4.747662000 & -0.587736000 \\
\hline & 6 & -1.175118000 & 5.154643000 & -0.085809000 \\
\hline & 6 & -2.109189000 & 4.219420000 & 0.348867000 \\
\hline & 6 & 0.854552000 & 0.347982000 & -0.799607000 \\
\hline & 6 & -0.365538000 & 0.995087000 & -0.175334000 \\
\hline & 1 & 1.380697000 & 3.094832000 & -1.046313000 \\
\hline & 1 & 0.790653000 & 5.497314000 & -0.922294000 \\
\hline & 1 & -1.418220000 & 6.212571000 & -0.040517000 \\
\hline & 1 & -3.075737000 & 4.534352000 & 0.730866000 \\
\hline & 16 & -2.773642000 & 1.501845000 & 0.731796000 \\
\hline & 8 & 1.078665000 & -0.455370000 & 2.832905000 \\
\hline & 8 & 0.898319000 & 0.236773000 & -2.036231000 \\
\hline & 7 & 1.857251000 & 0.033652000 & 0.036333000 \\
\hline
\end{tabular}




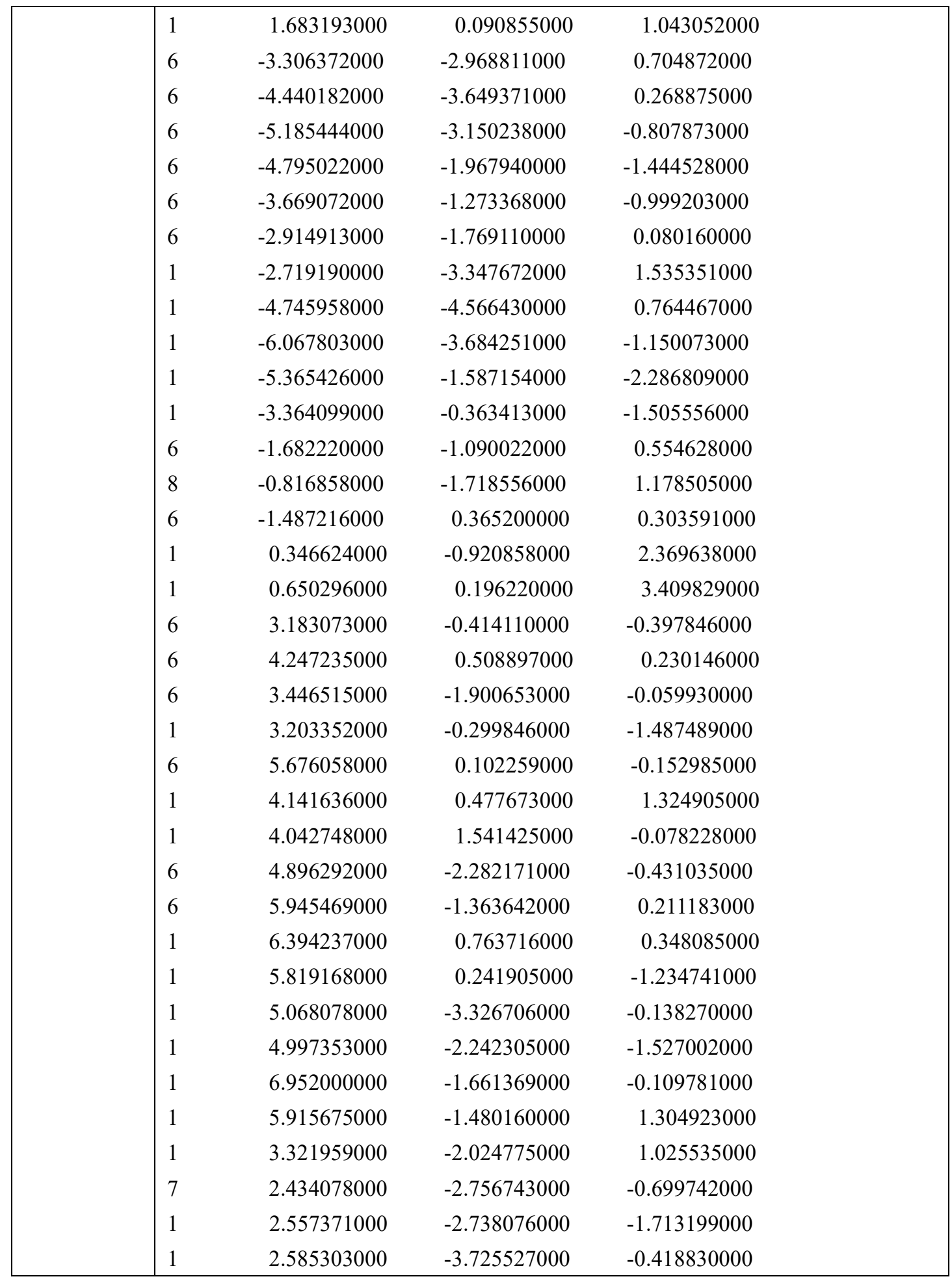

Table S2. Cartesian coordinates of key stationary points involved in the selectivity issues, optimized at the B3LYP-PCM/6-31+G* level of theory in DMF.

\begin{tabular}{l|rrrr}
\hline & 6 & 3.748437000 & 0.072172000 & 0.244444000 \\
TS-10-syn & 6 & 2.942233000 & -0.846228000 & -0.479894000 \\
& 6 & 3.574126000 & -1.848584000 & -1.242413000 \\
& 6 & 4.963897000 & -1.914014000 & -1.269072000 \\
& 6 & 5.746253000 & -0.994477000 & -0.542863000 \\
\hline
\end{tabular}




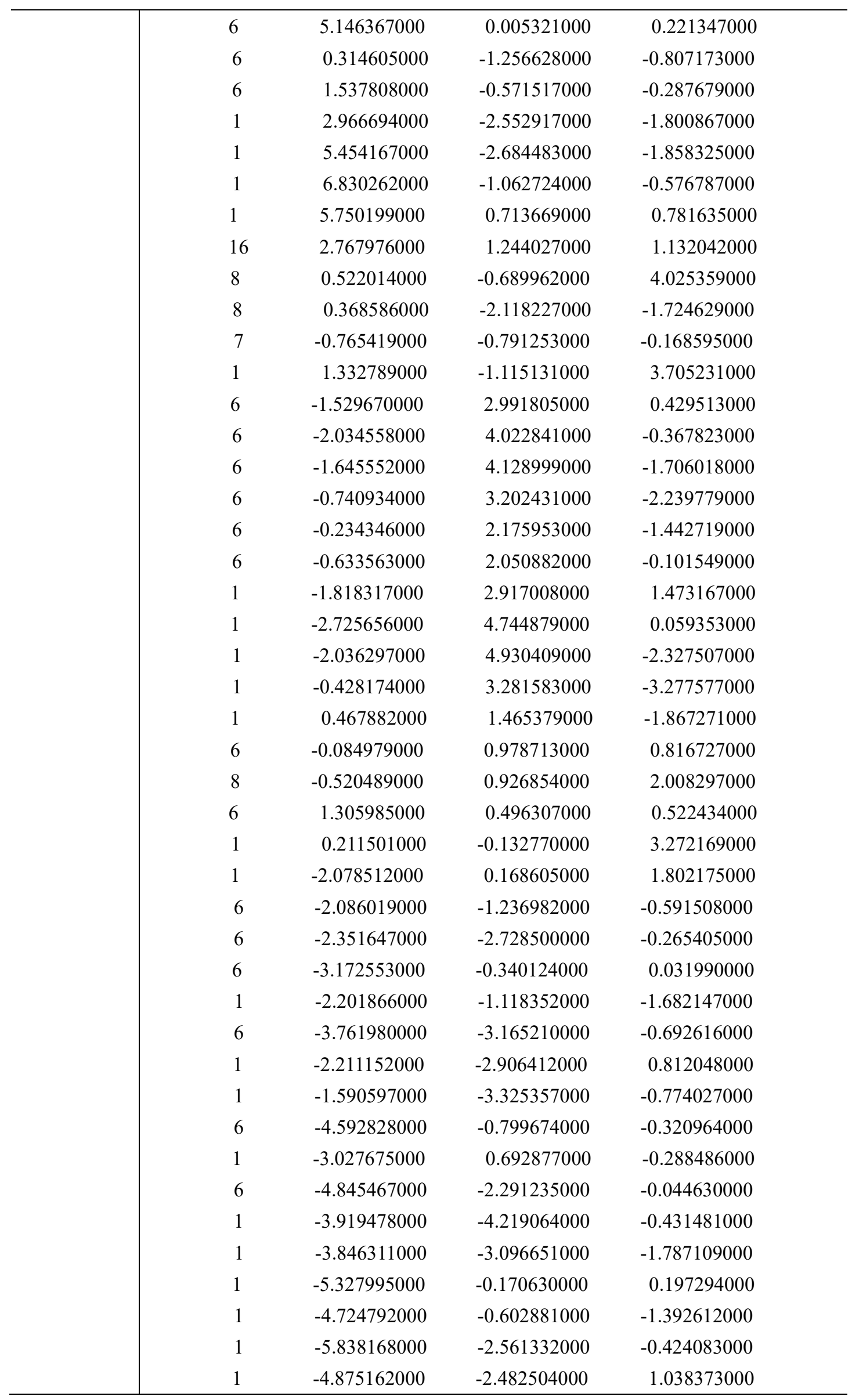




\begin{tabular}{|c|c|c|c|c|}
\hline & 7 & -2.987360000 & -0.288286000 & 1.543447000 \\
\hline & 1 & -3.740661000 & 0.246838000 & 1.986239000 \\
\hline & 1 & -2.995421000 & -1.220531000 & 1.965630000 \\
\hline \multirow{41}{*}{ TS-10-anti } & 6 & 3.620987000 & -0.340156000 & -0.089632000 \\
\hline & 6 & 2.582306000 & -1.238806000 & -0.446002000 \\
\hline & 6 & 2.934791000 & -2.493270000 & -0.985673000 \\
\hline & 6 & 4.277590000 & -2.816728000 & -1.153227000 \\
\hline & 6 & 5.292752000 & -1.909275000 & -0.790525000 \\
\hline & 6 & 4.973512000 & -0.662835000 & -0.255742000 \\
\hline & 6 & -0.076096000 & -1.320165000 & -0.420507000 \\
\hline & 6 & 1.270397000 & -0.677123000 & -0.194239000 \\
\hline & 1 & 2.148064000 & -3.188982000 & -1.255201000 \\
\hline & 1 & 4.548989000 & -3.783651000 & -1.569033000 \\
\hline & 1 & 6.335896000 & -2.180813000 & -0.928921000 \\
\hline & 1 & 5.754528000 & 0.039232000 & 0.022593000 \\
\hline & 16 & 2.977812000 & 1.167403000 & 0.554667000 \\
\hline & 8 & 1.091634000 & -0.211456000 & 3.985990000 \\
\hline & 8 & -0.149045000 & -2.580362000 & -0.546226000 \\
\hline & 7 & -1.042805000 & -0.409049000 & -0.411686000 \\
\hline & 1 & 1.883111000 & -0.630433000 & 3.613396000 \\
\hline & 6 & -1.234601000 & 3.448530000 & 0.530130000 \\
\hline & 6 & -1.665620000 & 4.553979000 & -0.203173000 \\
\hline & 6 & -1.174348000 & 4.772051000 & -1.495999000 \\
\hline & 6 & -0.250508000 & 3.879714000 & -2.052934000 \\
\hline & 6 & 0.180493000 & 2.773322000 & -1.320896000 \\
\hline & 6 & -0.309260000 & 2.546928000 & -0.023375000 \\
\hline & 1 & -1.603720000 & 3.274884000 & 1.535966000 \\
\hline & 1 & -2.379943000 & 5.247488000 & 0.232382000 \\
\hline & 1 & -1.508689000 & 5.634577000 & -2.066523000 \\
\hline & 1 & 0.131322000 & 4.045763000 & -3.056533000 \\
\hline & 1 & 0.887583000 & 2.075860000 & -1.759452000 \\
\hline & 6 & 0.151026000 & 1.389113000 & 0.790188000 \\
\hline & 8 & -0.266549000 & 1.225019000 & 1.957559000 \\
\hline & 6 & 1.333060000 & 0.581111000 & 0.330311000 \\
\hline & 1 & 0.675951000 & 0.261290000 & 3.231164000 \\
\hline & 6 & -2.443139000 & -0.799663000 & -0.456479000 \\
\hline & 6 & -2.879944000 & -1.598653000 & -1.706489000 \\
\hline & 6 & -2.858562000 & -1.560215000 & 0.821985000 \\
\hline & 1 & -3.023640000 & 0.138546000 & -0.466273000 \\
\hline & 6 & -4.376444000 & -1.945197000 & -1.669918000 \\
\hline & 1 & -2.283039000 & -2.514106000 & -1.767620000 \\
\hline & 1 & -2.644458000 & -0.997075000 & -2.593387000 \\
\hline & 6 & -4.342235000 & -1.932806000 & 0.865681000 \\
\hline & 6 & -4.736506000 & -2.721664000 & -0.394285000 \\
\hline
\end{tabular}




\begin{tabular}{|c|c|c|c|c|}
\hline & 1 & -4.643419000 & -2.534929000 & -2.556223000 \\
\hline & 1 & -4.974740000 & -1.022597000 & -1.713749000 \\
\hline & 1 & -4.561231000 & -2.516031000 & 1.769242000 \\
\hline & 1 & -4.946860000 & -1.014945000 & 0.918305000 \\
\hline & 1 & -5.811225000 & -2.937231000 & -0.361673000 \\
\hline & 1 & -4.218510000 & -3.691220000 & -0.395287000 \\
\hline & 7 & -2.516370000 & -0.679574000 & 2.012551000 \\
\hline & 1 & -2.494371000 & -1.206453000 & 2.890984000 \\
\hline & 1 & -3.205621000 & 0.070776000 & 2.128169000 \\
\hline & 1 & -1.596216000 & -0.217335000 & 1.886738000 \\
\hline & 1 & -2.226764000 & -2.445469000 & 0.926418000 \\
\hline \multirow{33}{*}{ INT-10-syn } & 6 & 3.679969000 & 0.076265000 & 0.297909000 \\
\hline & 6 & 2.999093000 & -0.848608000 & -0.542012000 \\
\hline & 6 & 3.750088000 & -1.754999000 & -1.312320000 \\
\hline & 6 & 5.139350000 & -1.727691000 & -1.231816000 \\
\hline & 6 & 5.797637000 & -0.808399000 & -0.392457000 \\
\hline & 6 & 5.075739000 & 0.100461000 & 0.381089000 \\
\hline & 6 & 0.346234000 & -1.275160000 & -0.966275000 \\
\hline & 6 & 1.577560000 & -0.664489000 & -0.421243000 \\
\hline & 1 & 3.242412000 & -2.465734000 & -1.957200000 \\
\hline & 1 & 5.725074000 & -2.425819000 & -1.823501000 \\
\hline & 1 & 6.883026000 & -0.804710000 & -0.344339000 \\
\hline & 1 & 5.587159000 & 0.807488000 & 1.027940000 \\
\hline & 16 & 2.556522000 & 1.129662000 & 1.185806000 \\
\hline & 8 & 0.241360000 & -1.688922000 & 3.522246000 \\
\hline & 8 & 0.257547000 & -2.210663000 & -1.768351000 \\
\hline & 7 & -0.718556000 & -0.602610000 & -0.398376000 \\
\hline & 1 & 1.198966000 & -1.548244000 & 3.585479000 \\
\hline & 6 & -0.943094000 & 2.953165000 & 0.807115000 \\
\hline & 6 & -1.219007000 & 4.230293000 & 0.305406000 \\
\hline & 6 & -1.201649000 & 4.465104000 & -1.071722000 \\
\hline & 6 & -0.905220000 & 3.413719000 & -1.946785000 \\
\hline & 6 & -0.632143000 & 2.139126000 & -1.446209000 \\
\hline & 6 & -0.657122000 & 1.895852000 & -0.065442000 \\
\hline & 1 & -0.955498000 & 2.777178000 & 1.877718000 \\
\hline & 1 & -1.446207000 & 5.039347000 & 0.994843000 \\
\hline & 1 & -1.416107000 & 5.456749000 & -1.461338000 \\
\hline & 1 & -0.888649000 & 3.584212000 & -3.020112000 \\
\hline & 1 & -0.408071000 & 1.329208000 & -2.134559000 \\
\hline & 6 & -0.281408000 & 0.508018000 & 0.499503000 \\
\hline & 8 & -0.738344000 & 0.338440000 & 1.819767000 \\
\hline & 6 & 1.219627000 & 0.318712000 & 0.441584000 \\
\hline & 1 & -0.075424000 & -0.998962000 & 2.897008000 \\
\hline & 1 & -1.756186000 & 0.364048000 & 1.746909000 \\
\hline
\end{tabular}




\begin{tabular}{|c|c|c|c|c|}
\hline & 6 & -2.091233000 & -1.125784000 & -0.597856000 \\
\hline & 6 & -2.327231000 & -2.450008000 & 0.159007000 \\
\hline & 6 & -3.242268000 & -0.131763000 & -0.321481000 \\
\hline & 1 & -2.142778000 & -1.352577000 & -1.669604000 \\
\hline & 6 & -3.646539000 & -3.094341000 & -0.302750000 \\
\hline & 1 & -2.344729000 & -2.264818000 & 1.241338000 \\
\hline & 1 & -1.491639000 & -3.129737000 & -0.032915000 \\
\hline & 6 & -4.561489000 & -0.772200000 & -0.812108000 \\
\hline & 1 & -3.062776000 & 0.786225000 & -0.886743000 \\
\hline & 6 & -4.839562000 & -2.135988000 & -0.154842000 \\
\hline & 1 & -3.829266000 & -4.016882000 & 0.262115000 \\
\hline & 1 & -3.546019000 & -3.386929000 & -1.358485000 \\
\hline & 1 & -5.386667000 & -0.075605000 & -0.616895000 \\
\hline & 1 & -4.504153000 & -0.896804000 & -1.902210000 \\
\hline & 1 & -5.739392000 & -2.578031000 & -0.600270000 \\
\hline & 1 & -5.071570000 & -2.001965000 & 0.912123000 \\
\hline & 7 & -3.302422000 & 0.266369000 & 1.112189000 \\
\hline & 1 & -3.833059000 & 1.131885000 & 1.203046000 \\
\hline & 1 & -3.804455000 & -0.428967000 & 1.662985000 \\
\hline \multirow{25}{*}{ INT-10-anti } & 6 & 3.672107000 & -0.087080000 & 0.045728000 \\
\hline & 6 & 2.867470000 & -1.187756000 & -0.361286000 \\
\hline & 6 & 3.494911000 & -2.365157000 & -0.807909000 \\
\hline & 6 & 4.885203000 & -2.425373000 & -0.839506000 \\
\hline & 6 & 5.666747000 & -1.327205000 & -0.431780000 \\
\hline & 6 & 5.068914000 & -0.148902000 & 0.014889000 \\
\hline & 6 & 0.168568000 & -1.513171000 & -0.443850000 \\
\hline & 6 & 1.475051000 & -0.851634000 & -0.226548000 \\
\hline & 1 & 2.893154000 & -3.212716000 & -1.121427000 \\
\hline & 1 & 5.375358000 & -3.332162000 & -1.183119000 \\
\hline & 1 & 6.750584000 & -1.395670000 & -0.464331000 \\
\hline & 1 & 5.674628000 & 0.696211000 & 0.329100000 \\
\hline & 16 & 2.693517000 & 1.299993000 & 0.571777000 \\
\hline & 8 & 0.572265000 & 2.304219000 & 3.877878000 \\
\hline & 8 & -0.033898000 & -2.672378000 & -0.816713000 \\
\hline & 7 & -0.799118000 & -0.574379000 & -0.140863000 \\
\hline & 1 & 0.141265000 & 3.172976000 & 3.878040000 \\
\hline & 6 & -1.344773000 & 2.984137000 & 0.180474000 \\
\hline & 6 & -1.743802000 & 4.089347000 & -0.582027000 \\
\hline & 6 & -1.485614000 & 4.128637000 & -1.953683000 \\
\hline & 6 & -0.824815000 & 3.054836000 & -2.563774000 \\
\hline & 6 & -0.426523000 & 1.954677000 & -1.803740000 \\
\hline & 6 & -0.685694000 & 1.909487000 & -0.424920000 \\
\hline & 1 & -1.549096000 & 2.953103000 & 1.244641000 \\
\hline & 1 & -2.257084000 & 4.916731000 & -0.098713000 \\
\hline
\end{tabular}




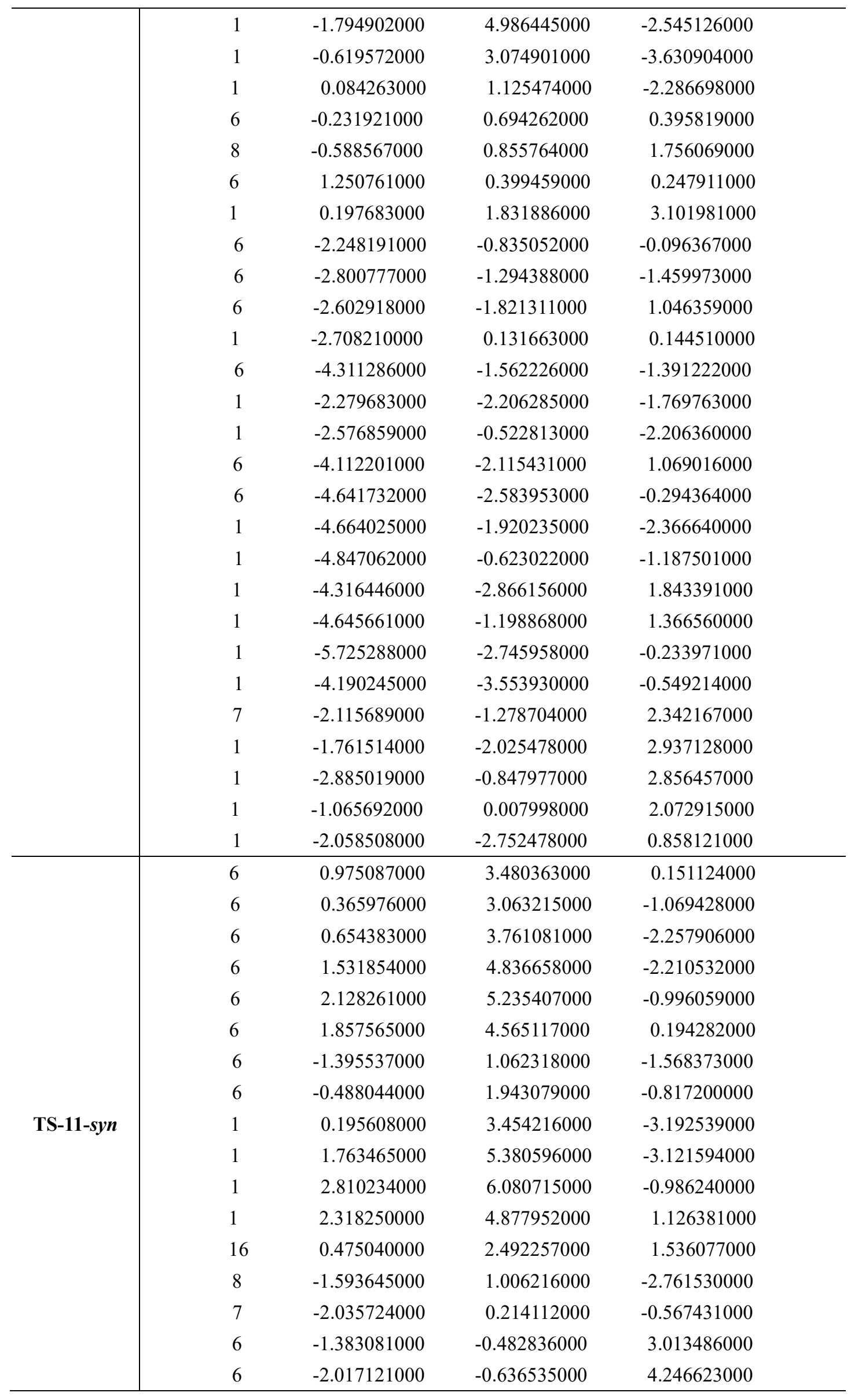




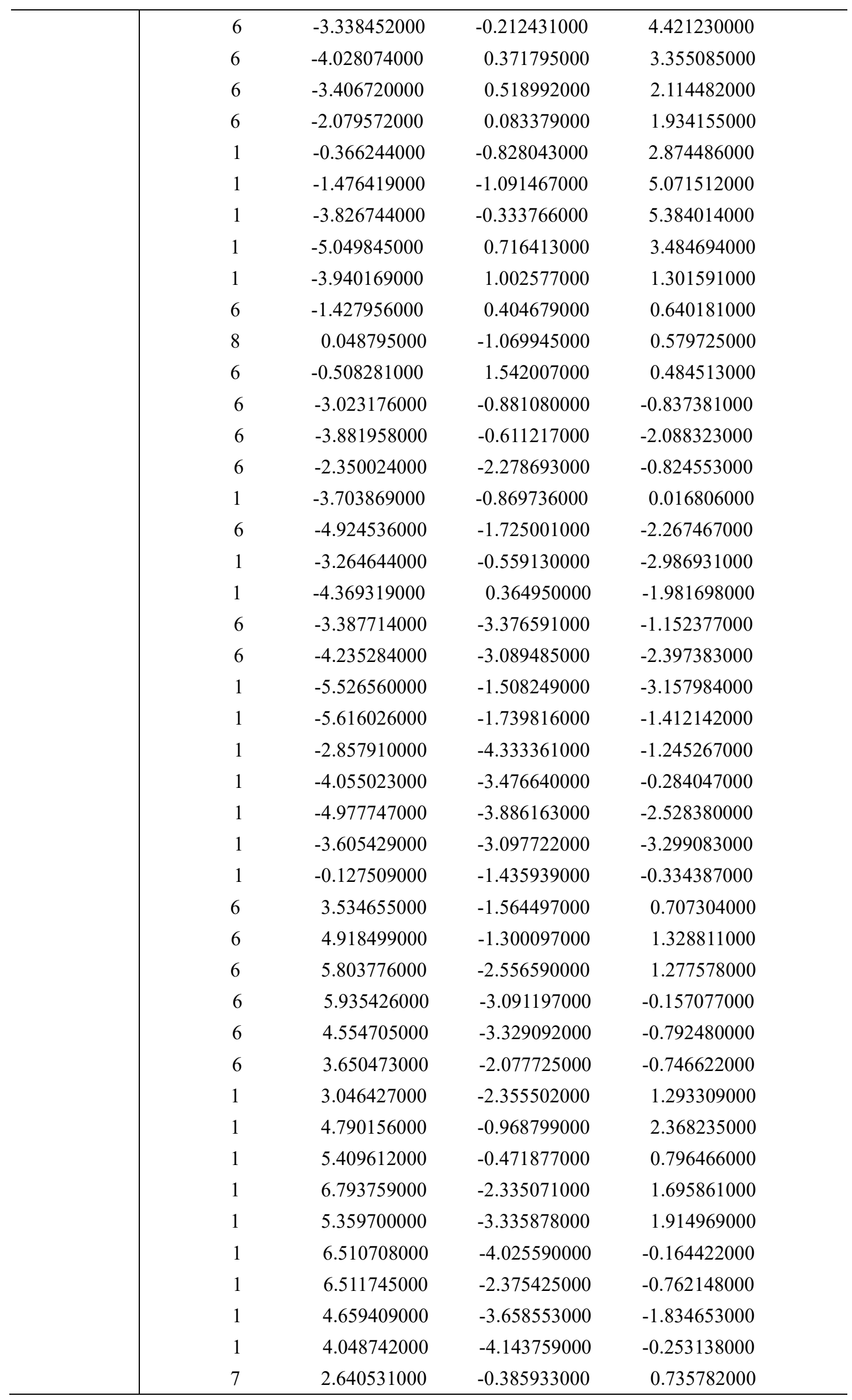




\begin{tabular}{|c|c|c|c|c|}
\hline & 1 & 2.940400000 & 0.249720000 & -0.007116000 \\
\hline & 1 & 2.745662000 & 0.116296000 & 1.617938000 \\
\hline & 1 & 1.034426000 & -0.751076000 & 0.597740000 \\
\hline & 1 & -2.008028000 & -2.466929000 & 0.199468000 \\
\hline & 7 & -1.125782000 & -2.270132000 & -1.651636000 \\
\hline & 1 & -1.311722000 & -1.968692000 & -2.607903000 \\
\hline & 1 & -0.748318000 & -3.215114000 & -1.719897000 \\
\hline & 1 & 2.644776000 & -2.361555000 & -1.080466000 \\
\hline & 7 & 4.070113000 & -0.973225000 & -1.635751000 \\
\hline & 1 & 3.875896000 & -1.221108000 & -2.604371000 \\
\hline & 1 & 5.075984000 & -0.818669000 & -1.582781000 \\
\hline \multirow{33}{*}{ TS-11-anti } & 6 & 0.693853000 & 3.573821000 & 0.407292000 \\
\hline & 6 & -0.058923000 & 3.370314000 & -0.786627000 \\
\hline & 6 & -0.015644000 & 4.342276000 & -1.804162000 \\
\hline & 6 & 0.766987000 & 5.474778000 & -1.619352000 \\
\hline & 6 & 1.509769000 & 5.659616000 & -0.434598000 \\
\hline & 6 & 1.482457000 & 4.715153000 & 0.588528000 \\
\hline & 6 & -1.740444000 & 1.359829000 & -1.477615000 \\
\hline & 6 & -0.765423000 & 2.128959000 & -0.698150000 \\
\hline & 1 & -0.586468000 & 4.199386000 & -2.716350000 \\
\hline & 1 & 0.809358000 & 6.230551000 & -2.398129000 \\
\hline & 1 & 2.113946000 & 6.554271000 & -0.315303000 \\
\hline & 1 & 2.054741000 & 4.864275000 & 1.498922000 \\
\hline & 16 & 0.492023000 & 2.253173000 & 1.573619000 \\
\hline & 8 & -2.220635000 & 1.570860000 & -2.568825000 \\
\hline & 7 & -2.108667000 & 0.211977000 & -0.645938000 \\
\hline & 6 & -0.982412000 & -1.148387000 & 2.622156000 \\
\hline & 6 & -1.415896000 & -1.522724000 & 3.895582000 \\
\hline & 6 & -2.653693000 & -1.089864000 & 4.377500000 \\
\hline & 6 & -3.457817000 & -0.271449000 & 3.579468000 \\
\hline & 6 & -3.039304000 & 0.092203000 & 2.299919000 \\
\hline & 6 & -1.802380000 & -0.360932000 & 1.800345000 \\
\hline & 1 & -0.011896000 & -1.476016000 & 2.273281000 \\
\hline & 1 & -0.777972000 & -2.150788000 & 4.510537000 \\
\hline & 1 & -2.985772000 & -1.380663000 & 5.370038000 \\
\hline & 1 & -4.414327000 & 0.087255000 & 3.948465000 \\
\hline & 1 & -3.662171000 & 0.746269000 & 1.699946000 \\
\hline & 6 & -1.368772000 & 0.217457000 & 0.495139000 \\
\hline & 8 & 0.258462000 & -0.990458000 & -0.059165000 \\
\hline & 6 & -0.557209000 & 1.450565000 & 0.465261000 \\
\hline & 1 & 0.097805000 & -1.929237000 & 0.141797000 \\
\hline & 6 & -3.016881000 & -0.818985000 & -1.236988000 \\
\hline & 6 & -2.253557000 & -2.085036000 & -1.728092000 \\
\hline & 6 & -4.235954000 & -1.171466000 & -0.368322000 \\
\hline
\end{tabular}




\begin{tabular}{|c|c|c|c|c|}
\hline & 1 & -3.387221000 & -0.306159000 & -2.130435000 \\
\hline & 6 & -3.243500000 & -3.013195000 & -2.457449000 \\
\hline & 1 & -1.873668000 & -2.621414000 & -0.847983000 \\
\hline & 6 & -5.196186000 & -2.084117000 & -1.147311000 \\
\hline & 6 & -4.482526000 & -3.355732000 & -1.621557000 \\
\hline & 1 & -2.703552000 & -3.922669000 & -2.745209000 \\
\hline & 1 & -3.556699000 & -2.523188000 & -3.392362000 \\
\hline & 1 & -6.050826000 & -2.336251000 & -0.507768000 \\
\hline & 1 & -5.598533000 & -1.540825000 & -2.014767000 \\
\hline & 1 & -5.165613000 & -3.980874000 & -2.209959000 \\
\hline & 1 & -4.182392000 & -3.952499000 & -0.747722000 \\
\hline & 6 & 3.842332000 & -1.334586000 & -1.490837000 \\
\hline & 6 & 4.821065000 & -2.364771000 & -2.075645000 \\
\hline & 6 & 6.156297000 & -2.361341000 & -1.315685000 \\
\hline & 6 & 5.938978000 & -2.575545000 & 0.190683000 \\
\hline & 6 & 4.964568000 & -1.543915000 & 0.788997000 \\
\hline & 6 & 3.631407000 & -1.549037000 & 0.012719000 \\
\hline & 1 & 4.985087000 & -2.154261000 & -3.139710000 \\
\hline & 1 & 4.371300000 & -3.366833000 & -2.018288000 \\
\hline & 1 & 6.821741000 & -3.138561000 & -1.712392000 \\
\hline & 1 & 6.665233000 & -1.399389000 & -1.477044000 \\
\hline & 1 & 6.893693000 & -2.522343000 & 0.730051000 \\
\hline & 1 & 5.533533000 & -3.584575000 & 0.363876000 \\
\hline & 1 & 5.405952000 & -0.541882000 & 0.683565000 \\
\hline & 1 & 3.154792000 & -2.528273000 & 0.167880000 \\
\hline & 7 & 2.688072000 & -0.538114000 & 0.559462000 \\
\hline & 1 & 2.955446000 & 0.396626000 & 0.243145000 \\
\hline & 1 & 2.799966000 & -0.535564000 & 1.578384000 \\
\hline & 1 & 2.875950000 & -1.383887000 & -2.007857000 \\
\hline & 1 & 1.323656000 & -0.774529000 & 0.236123000 \\
\hline & 7 & 4.680427000 & -1.724728000 & 2.224668000 \\
\hline & 1 & 5.504524000 & -1.492855000 & 2.777297000 \\
\hline & 1 & 4.475567000 & -2.705803000 & 2.420011000 \\
\hline & 1 & 4.240337000 & -0.320852000 & -1.653407000 \\
\hline & 1 & -3.921206000 & -1.685788000 & 0.545576000 \\
\hline & 7 & -1.110508000 & -1.821079000 & -2.606180000 \\
\hline & 1 & -0.399444000 & -1.284989000 & -2.110560000 \\
\hline & 1 & -1.401322000 & -1.279052000 & -3.419623000 \\
\hline & 1 & -4.750952000 & -0.252334000 & -0.069295000 \\
\hline \multirow{5}{*}{ INT-11-syn } & 6 & 3.664941000 & 0.377110000 & 0.092550000 \\
\hline & 6 & 3.002195000 & -0.873869000 & -0.118737000 \\
\hline & 6 & 3.767970000 & -2.051896000 & -0.252416000 \\
\hline & 6 & 5.147645000 & -1.964369000 & -0.166314000 \\
\hline & 6 & 5.787480000 & -0.722230000 & 0.053417000 \\
\hline
\end{tabular}




\begin{tabular}{|c|c|c|c|c|}
\hline & 6 & 5.059789000 & 0.455050000 & 0.187367000 \\
\hline & 6 & 0.386308000 & -1.445330000 & -0.459383000 \\
\hline & 6 & 1.596704000 & -0.666635000 & -0.144848000 \\
\hline & 1 & 3.274189000 & -3.004477000 & -0.414738000 \\
\hline & 1 & 5.750875000 & -2.861634000 & -0.265830000 \\
\hline & 1 & 6.870951000 & -0.685674000 & 0.117357000 \\
\hline & 1 & 5.560124000 & 1.403912000 & 0.353301000 \\
\hline & 16 & 2.543587000 & 1.734039000 & 0.203565000 \\
\hline & 8 & 0.267050000 & -2.597078000 & -0.788164000 \\
\hline & 7 & -0.723482000 & -0.463513000 & -0.417703000 \\
\hline & 6 & -0.618118000 & 2.982332000 & 0.820346000 \\
\hline & 6 & -1.289756000 & 4.200943000 & 0.845186000 \\
\hline & 6 & -2.270561000 & 4.479673000 & -0.113530000 \\
\hline & 6 & -2.587014000 & 3.533646000 & -1.096878000 \\
\hline & 6 & -1.946697000 & 2.299146000 & -1.111530000 \\
\hline & 6 & -0.955699000 & 2.009227000 & -0.146673000 \\
\hline & 1 & 0.131710000 & 2.765134000 & 1.574454000 \\
\hline & 1 & -1.045827000 & 4.933841000 & 1.607818000 \\
\hline & 1 & -2.782523000 & 5.437426000 & -0.100787000 \\
\hline & 1 & -3.329348000 & 3.761727000 & -1.855375000 \\
\hline & 1 & -2.169284000 & 1.586990000 & -1.899278000 \\
\hline & 6 & -0.217381000 & 0.758405000 & -0.171265000 \\
\hline & 6 & 1.214014000 & 0.640847000 & 0.021234000 \\
\hline & 6 & -2.156689000 & -0.849409000 & -0.257068000 \\
\hline & 6 & -2.620711000 & -1.905845000 & -1.275070000 \\
\hline & 6 & -2.437736000 & -1.223869000 & 1.222671000 \\
\hline & 1 & -2.725906000 & 0.058274000 & -0.455575000 \\
\hline & 6 & -4.121129000 & -2.182398000 & -1.079211000 \\
\hline & 1 & -2.059382000 & -2.835712000 & -1.157862000 \\
\hline & 1 & -2.423335000 & -1.538221000 & -2.289141000 \\
\hline & 6 & -3.937964000 & -1.572620000 & 1.371494000 \\
\hline & 6 & -4.420159000 & -2.624638000 & 0.360895000 \\
\hline & 1 & -4.439775000 & -2.952200000 & -1.792069000 \\
\hline & 1 & -4.699451000 & -1.276229000 & -1.312524000 \\
\hline & 1 & -4.114156000 & -1.908933000 & 2.401105000 \\
\hline & 1 & -4.524243000 & -0.651645000 & 1.237598000 \\
\hline & 1 & -5.496174000 & -2.792433000 & 0.491294000 \\
\hline & 1 & -3.932298000 & -3.590939000 & 0.556292000 \\
\hline & 1 & -2.238091000 & -0.326823000 & 1.822186000 \\
\hline & 7 & -1.485806000 & -2.236729000 & 1.697475000 \\
\hline & 1 & -1.663791000 & -3.147527000 & 1.275549000 \\
\hline & 1 & -1.597642000 & -2.360783000 & 2.702683000 \\
\hline \multirow{2}{*}{ INT-11-anti } & 6 & 3.739574000 & 0.470311000 & -0.050343000 \\
\hline & 6 & 3.187028000 & -0.849245000 & -0.017890000 \\
\hline
\end{tabular}




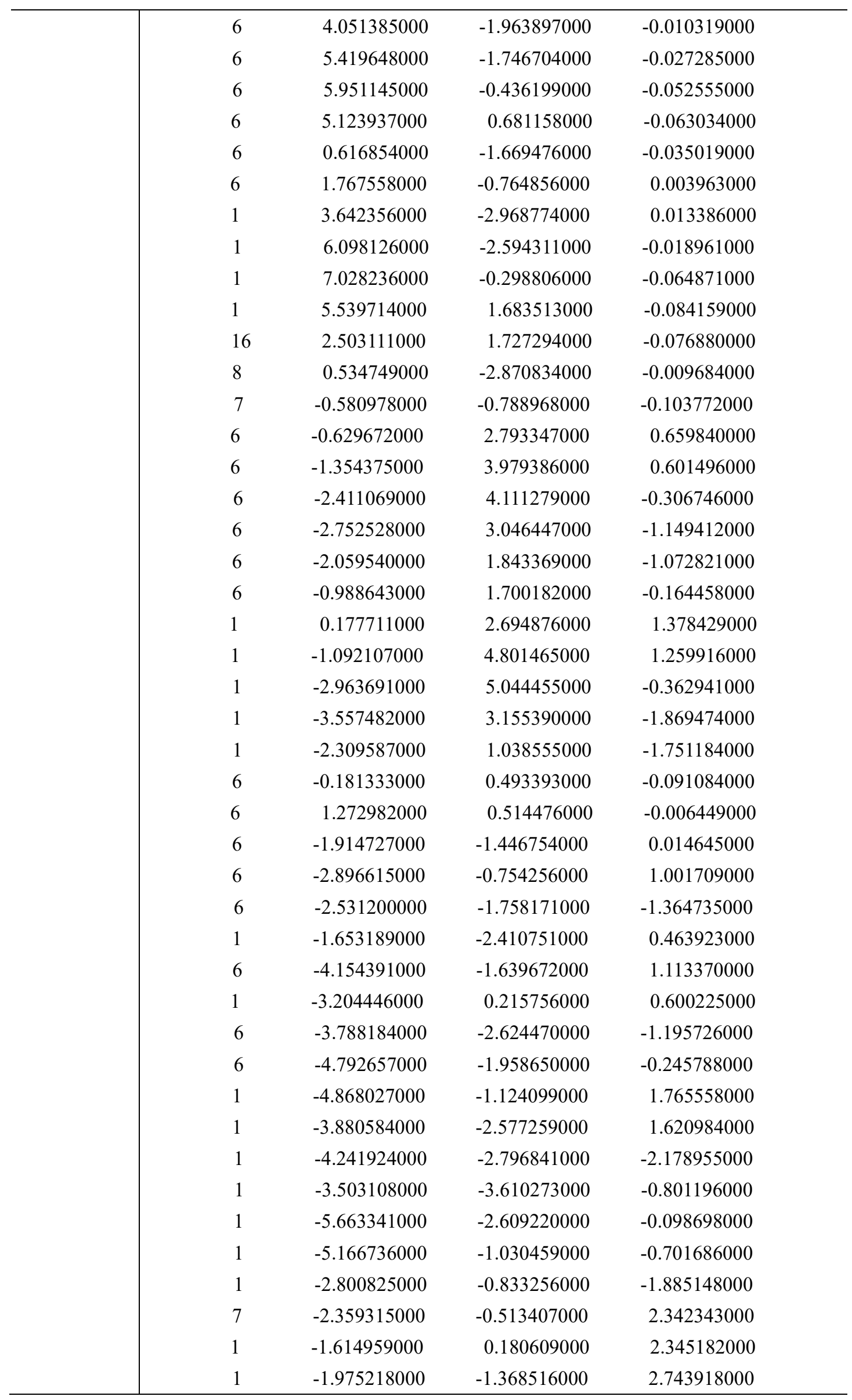




\begin{tabular}{|c|c|c|c|c|}
\hline & 1 & -1.787683000 & -2.271432000 & -1.984855000 \\
\hline & 6 & -3.545487000 & 0.479672000 & -0.396649000 \\
\hline & 6 & -3.040258000 & -0.738266000 & 0.154891000 \\
\hline & 6 & -3.936897000 & -1.777385000 & 0.481618000 \\
\hline & 6 & -5.290147000 & -1.591333000 & 0.246127000 \\
\hline & 6 & -5.772836000 & -0.386117000 & -0.312237000 \\
\hline & 6 & -4.912162000 & 0.656541000 & -0.641283000 \\
\hline & 6 & -0.536971000 & -1.450289000 & 0.868032000 \\
\hline & 6 & -1.623939000 & -0.659170000 & 0.264547000 \\
\hline & 1 & -3.561999000 & -2.704367000 & 0.903265000 \\
\hline & 1 & -5.992466000 & -2.382763000 & 0.489761000 \\
\hline & 1 & -6.838336000 & -0.268720000 & -0.486583000 \\
\hline & 1 & -5.293393000 & 1.579928000 & -1.065963000 \\
\hline & 16 & -2.274363000 & 1.668419000 & -0.699869000 \\
\hline & 8 & -0.600846000 & -2.505217000 & 1.455078000 \\
\hline & 7 & 0.676272000 & -0.650245000 & 0.707325000 \\
\hline & 6 & 0.936205000 & 2.824261000 & -0.638132000 \\
\hline & 6 & 1.618548000 & 4.023464000 & -0.470953000 \\
\hline & 6 & 2.444092000 & 4.212421000 & 0.646527000 \\
\hline & 6 & 2.595238000 & 3.193222000 & 1.593318000 \\
\hline & 6 & 1.936230000 & 1.979052000 & 1.421248000 \\
\hline & 6 & 1.097241000 & 1.783701000 & 0.302512000 \\
\hline \multirow[t]{22}{*}{ TS-12-syn } & 1 & 0.318808000 & 2.673899000 & -1.518506000 \\
\hline & 1 & 1.509538000 & 4.812694000 & -1.208322000 \\
\hline & 1 & 2.966672000 & 5.155387000 & 0.778512000 \\
\hline & 1 & 3.225035000 & 3.344837000 & 2.464467000 \\
\hline & 1 & 2.035171000 & 1.197153000 & 2.166988000 \\
\hline & 6 & 0.322381000 & 0.561962000 & 0.186565000 \\
\hline & 6 & -1.086243000 & 0.530307000 & -0.156383000 \\
\hline & 6 & 2.066546000 & -1.233243000 & 0.563482000 \\
\hline & 6 & 2.012995000 & -2.769642000 & 0.398740000 \\
\hline & 6 & 2.786000000 & -0.537001000 & -0.633853000 \\
\hline & 1 & 2.622764000 & -1.005941000 & 1.478114000 \\
\hline & 6 & 3.401231000 & -3.362480000 & 0.111458000 \\
\hline & 1 & 1.337318000 & -3.035038000 & -0.425143000 \\
\hline & 1 & 1.600775000 & -3.220586000 & 1.301499000 \\
\hline & 6 & 4.126296000 & -1.232089000 & -0.971612000 \\
\hline & 6 & 4.020941000 & -2.748249000 & -1.144976000 \\
\hline & 1 & 3.296561000 & -4.449082000 & 0.007540000 \\
\hline & 1 & 4.064550000 & -3.190614000 & 0.971666000 \\
\hline & 1 & 4.534535000 & -0.752069000 & -1.869653000 \\
\hline & 1 & 4.829586000 & -1.014882000 & -0.154629000 \\
\hline & 1 & 5.016105000 & -3.169122000 & -1.333381000 \\
\hline & 1 & 3.405742000 & -2.994860000 & -2.022479000 \\
\hline
\end{tabular}




\begin{tabular}{|c|c|c|c|c|}
\hline & 1 & 3.053315000 & 0.478300000 & -0.332553000 \\
\hline & 7 & 1.836094000 & -0.378125000 & -1.735786000 \\
\hline & 1 & 1.541928000 & -1.263121000 & -2.146241000 \\
\hline & 1 & 2.223784000 & 0.198565000 & -2.479778000 \\
\hline \multirow{40}{*}{ TS-12-anti } & 6 & 3.629545000 & 0.487613000 & 0.251386000 \\
\hline & 6 & 3.125358000 & -0.780565000 & -0.170991000 \\
\hline & 6 & 4.028730000 & -1.820930000 & -0.472722000 \\
\hline & 6 & 5.388626000 & -1.586452000 & -0.338399000 \\
\hline & 6 & 5.871243000 & -0.330222000 & 0.091780000 \\
\hline & 6 & 5.003264000 & 0.715220000 & 0.391981000 \\
\hline & 6 & 0.589563000 & -1.622115000 & -0.595105000 \\
\hline & 6 & 1.702485000 & -0.741210000 & -0.204526000 \\
\hline & 1 & 3.654984000 & -2.786601000 & -0.797844000 \\
\hline & 1 & 6.095932000 & -2.378784000 & -0.564111000 \\
\hline & 1 & 6.941767000 & -0.174631000 & 0.188025000 \\
\hline & 1 & 5.383496000 & 1.678334000 & 0.717740000 \\
\hline & 16 & 2.346669000 & 1.666950000 & 0.547505000 \\
\hline & 8 & 0.582089000 & -2.794678000 & -0.901425000 \\
\hline & 7 & -0.610142000 & -0.812246000 & -0.520735000 \\
\hline & 6 & -0.923584000 & 2.755813000 & 0.589171000 \\
\hline & 6 & -1.642597000 & 3.920873000 & 0.349839000 \\
\hline & 6 & -2.511022000 & 3.998656000 & -0.748836000 \\
\hline & 6 & -2.659997000 & 2.904696000 & -1.605883000 \\
\hline & 6 & -1.955719000 & 1.726075000 & -1.363401000 \\
\hline & 6 & -1.083005000 & 1.640333000 & -0.260582000 \\
\hline & 1 & -0.274583000 & 2.691809000 & 1.457717000 \\
\hline & 1 & -1.531522000 & 4.769081000 & 1.018410000 \\
\hline & 1 & -3.065755000 & 4.913607000 & -0.935652000 \\
\hline & 1 & -3.319815000 & 2.968580000 & -2.465774000 \\
\hline & 1 & -2.042026000 & 0.890622000 & -2.048736000 \\
\hline & 6 & -0.276080000 & 0.442552000 & -0.062781000 \\
\hline & 6 & 1.160082000 & 0.466607000 & 0.160654000 \\
\hline & 6 & -1.869646000 & -1.504035000 & -0.106377000 \\
\hline & 6 & -2.515831000 & -0.738885000 & 1.065866000 \\
\hline & 6 & -2.894368000 & -1.811416000 & -1.203148000 \\
\hline & 1 & -1.511321000 & -2.469697000 & 0.270570000 \\
\hline & 6 & -3.590166000 & -1.639328000 & 1.704924000 \\
\hline & 1 & -3.024743000 & 0.148708000 & 0.673413000 \\
\hline & 6 & -4.011438000 & -2.683831000 & -0.594474000 \\
\hline & 6 & -4.642199000 & -2.035737000 & 0.650571000 \\
\hline & 1 & -4.064848000 & -1.100924000 & 2.533728000 \\
\hline & 1 & -3.114324000 & -2.535251000 & 2.127957000 \\
\hline & 1 & -4.781505000 & -2.869250000 & -1.352352000 \\
\hline & 1 & -3.592710000 & -3.664252000 & -0.325869000 \\
\hline
\end{tabular}




\begin{tabular}{|c|c|c|c|c|}
\hline & 1 & -5.371530000 & -2.720707000 & 1.099389000 \\
\hline & 1 & -5.199655000 & -1.137279000 & 0.349130000 \\
\hline & 1 & -3.322523000 & -0.883680000 & -1.601235000 \\
\hline & 7 & -1.453230000 & -0.243016000 & 1.937097000 \\
\hline & 1 & -0.975769000 & -0.979197000 & 2.457488000 \\
\hline & 1 & -1.776398000 & 0.464970000 & 2.593745000 \\
\hline & 1 & -2.406428000 & -2.334680000 & -2.032615000 \\
\hline \multirow{37}{*}{ INT-12-syn } & 6 & -3.271507000 & 0.109862000 & -0.747891000 \\
\hline & 6 & -2.800722000 & -0.735413000 & 0.293485000 \\
\hline & 6 & -3.721403000 & -1.516656000 & 1.015884000 \\
\hline & 6 & -5.070812000 & -1.444697000 & 0.688378000 \\
\hline & 6 & -5.519971000 & -0.607915000 & -0.352443000 \\
\hline & 6 & -4.627832000 & 0.174563000 & -1.083278000 \\
\hline & 6 & -0.315821000 & -1.193983000 & 1.265812000 \\
\hline & 6 & -1.373568000 & -0.623929000 & 0.407101000 \\
\hline & 1 & -3.373047000 & -2.165009000 & 1.813667000 \\
\hline & 1 & -5.789842000 & -2.043113000 & 1.240354000 \\
\hline & 1 & -6.579075000 & -0.568789000 & -0.590628000 \\
\hline & 1 & -4.979264000 & 0.818763000 & -1.883511000 \\
\hline & 16 & -1.959874000 & 1.016929000 & -1.527366000 \\
\hline & 8 & -0.410468000 & -2.048924000 & 2.135811000 \\
\hline & 7 & 0.880454000 & -0.566688000 & 0.880906000 \\
\hline & 6 & 0.974001000 & 2.872525000 & -0.776732000 \\
\hline & 6 & 1.283654000 & 4.190231000 & -0.435214000 \\
\hline & 6 & 1.621421000 & 4.512566000 & 0.883187000 \\
\hline & 6 & 1.648205000 & 3.511441000 & 1.857124000 \\
\hline & 6 & 1.346597000 & 2.189356000 & 1.517680000 \\
\hline & 6 & 1.012210000 & 1.863402000 & 0.198357000 \\
\hline & 1 & 0.695597000 & 2.653777000 & -1.805626000 \\
\hline & 1 & 1.254232000 & 4.962326000 & -1.198434000 \\
\hline & 1 & 1.859604000 & 5.538757000 & 1.148363000 \\
\hline & 1 & 1.907656000 & 3.753843000 & 2.883734000 \\
\hline & 1 & 1.369188000 & 1.413176000 & 2.275143000 \\
\hline & 6 & 0.652954000 & 0.421674000 & -0.178099000 \\
\hline & 6 & -0.811442000 & 0.237695000 & -0.489947000 \\
\hline & 6 & 2.190128000 & -1.262943000 & 0.756680000 \\
\hline & 6 & 2.043216000 & -2.775584000 & 0.484796000 \\
\hline & 6 & 2.859351000 & -0.485415000 & -0.405513000 \\
\hline & 1 & 2.786803000 & -1.106001000 & 1.660595000 \\
\hline & 6 & 3.324820000 & -3.387183000 & -0.096717000 \\
\hline & 1 & 1.220887000 & -2.948204000 & -0.223026000 \\
\hline & 1 & 1.759518000 & -3.274696000 & 1.414292000 \\
\hline & 6 & 3.958396000 & -1.207929000 & -1.185263000 \\
\hline & 6 & 3.699758000 & -2.702176000 & -1.416069000 \\
\hline
\end{tabular}




\begin{tabular}{|c|c|c|c|c|}
\hline & 1 & 3.165532000 & -4.460032000 & -0.253040000 \\
\hline & 1 & 4.152792000 & -3.291667000 & 0.620185000 \\
\hline & 1 & 4.151586000 & -0.674950000 & -2.123994000 \\
\hline & 1 & 4.868525000 & -1.095281000 & -0.582595000 \\
\hline & 1 & 4.601690000 & -3.150907000 & -1.847202000 \\
\hline & 1 & 2.896998000 & -2.853093000 & -2.152056000 \\
\hline & 1 & 3.244742000 & 0.465734000 & -0.033637000 \\
\hline & 7 & 1.645803000 & -0.073057000 & -1.242074000 \\
\hline & 1 & 1.249960000 & -0.864284000 & -1.763385000 \\
\hline & 1 & 1.884054000 & 0.651450000 & -1.927547000 \\
\hline \multirow{34}{*}{ INT-12-anti } & 6 & 3.478091000 & 0.170153000 & 0.560993000 \\
\hline & 6 & 2.936301000 & -0.858879000 & -0.256188000 \\
\hline & 6 & 3.805818000 & -1.764089000 & -0.891865000 \\
\hline & 6 & 5.176443000 & -1.630429000 & -0.700148000 \\
\hline & 6 & 5.697171000 & -0.608575000 & 0.118272000 \\
\hline & 6 & 4.856583000 & 0.299668000 & 0.759268000 \\
\hline & 6 & 0.383332000 & -1.500625000 & -0.885744000 \\
\hline & 6 & 1.503333000 & -0.767676000 & -0.271082000 \\
\hline & 1 & 3.403373000 & -2.553794000 & -1.518509000 \\
\hline & 1 & 5.856546000 & -2.323944000 & -1.186204000 \\
\hline & 1 & 6.771709000 & -0.524099000 & 0.252931000 \\
\hline & 1 & 5.263196000 & 1.085848000 & 1.388138000 \\
\hline & 16 & 2.220728000 & 1.208299000 & 1.263464000 \\
\hline & 8 & 0.392475000 & -2.558384000 & -1.499041000 \\
\hline & 7 & -0.789922000 & -0.781360000 & -0.578116000 \\
\hline & 6 & -0.854231000 & 2.853691000 & 0.610493000 \\
\hline & 6 & -1.238497000 & 4.107059000 & 0.131236000 \\
\hline & 6 & -1.698854000 & 4.244834000 & -1.182332000 \\
\hline & 6 & -1.772933000 & 3.123742000 & -2.012414000 \\
\hline & 6 & -1.396167000 & 1.865389000 & -1.533969000 \\
\hline & 6 & -0.939871000 & 1.723663000 & -0.218643000 \\
\hline & 1 & -0.480297000 & 2.779563000 & 1.629949000 \\
\hline & 1 & -1.171577000 & 4.973248000 & 0.782935000 \\
\hline & 1 & -1.995252000 & 5.221082000 & -1.555353000 \\
\hline & 1 & -2.127533000 & 3.222358000 & -3.034419000 \\
\hline & 1 & -1.455311000 & 0.995872000 & -2.179383000 \\
\hline & 6 & -0.488690000 & 0.359646000 & 0.306637000 \\
\hline & 6 & 1.003520000 & 0.249359000 & 0.490363000 \\
\hline & 6 & -1.985404000 & -1.530085000 & -0.121144000 \\
\hline & 6 & -2.584137000 & -0.606712000 & 0.937285000 \\
\hline & 6 & -3.076883000 & -1.920273000 & -1.116803000 \\
\hline & 1 & -1.628051000 & -2.452148000 & 0.359807000 \\
\hline & 6 & -3.581102000 & -1.326320000 & 1.829127000 \\
\hline & 1 & -3.076963000 & 0.240528000 & 0.450613000 \\
\hline
\end{tabular}




\begin{tabular}{|c|c|c|c|c|}
\hline & 6 & -4.183765000 & -2.644753000 & -0.314711000 \\
\hline & 6 & -4.706562000 & -1.830231000 & 0.888762000 \\
\hline & 1 & -3.992918000 & -0.651798000 & 2.587183000 \\
\hline & 1 & -3.101592000 & -2.166963000 & 2.345730000 \\
\hline & 1 & -5.020071000 & -2.882270000 & -0.981652000 \\
\hline & 1 & -3.787094000 & -3.604925000 & 0.043598000 \\
\hline & 1 & -5.407729000 & -2.438665000 & 1.470177000 \\
\hline & 1 & -5.271113000 & -0.961405000 & 0.523862000 \\
\hline & 1 & -3.474230000 & -1.027204000 & -1.615492000 \\
\hline & 7 & -1.339800000 & 0.005064000 & 1.556732000 \\
\hline & 1 & -0.844940000 & -0.663375000 & 2.160170000 \\
\hline & 1 & -1.554873000 & 0.829592000 & 2.126719000 \\
\hline & 1 & -2.669651000 & -2.580487000 & -1.888558000 \\
\hline \multirow{31}{*}{ 4-syn } & 6 & -3.224360000 & 0.053298000 & -0.790261000 \\
\hline & 6 & -2.760249000 & -0.732259000 & 0.299717000 \\
\hline & 6 & -3.687891000 & -1.479681000 & 1.047773000 \\
\hline & 6 & -5.035413000 & -1.434248000 & 0.701389000 \\
\hline & 6 & -5.477120000 & -0.655755000 & -0.385557000 \\
\hline & 6 & -4.576738000 & 0.092805000 & -1.143588000 \\
\hline & 6 & -0.275374000 & -1.144528000 & 1.306295000 \\
\hline & 6 & -1.332323000 & -0.603272000 & 0.427648000 \\
\hline & 1 & -3.346854000 & -2.083901000 & 1.882845000 \\
\hline & 1 & -5.756951000 & -2.008552000 & 1.275926000 \\
\hline & 1 & -6.533338000 & -0.634904000 & -0.639321000 \\
\hline & 1 & -4.920682000 & 0.692539000 & -1.981301000 \\
\hline & 16 & -1.901102000 & 0.920841000 & -1.599149000 \\
\hline & 8 & -0.388883000 & -1.979443000 & 2.206103000 \\
\hline & 7 & 0.914189000 & -0.546082000 & 0.908729000 \\
\hline & 6 & 1.007385000 & 2.857190000 & -0.810455000 \\
\hline & 6 & 1.258879000 & 4.191525000 & -0.482933000 \\
\hline & 6 & 1.500423000 & 4.554933000 & 0.846545000 \\
\hline & 6 & 1.485984000 & 3.573774000 & 1.841068000 \\
\hline & 6 & 1.234647000 & 2.237064000 & 1.511158000 \\
\hline & 6 & 0.997291000 & 1.864506000 & 0.182511000 \\
\hline & 1 & 0.816282000 & 2.601093000 & -1.850825000 \\
\hline & 1 & 1.263307000 & 4.945369000 & -1.265797000 \\
\hline & 1 & 1.697393000 & 5.592586000 & 1.102564000 \\
\hline & 1 & 1.674255000 & 3.843737000 & 2.877089000 \\
\hline & 1 & 1.228367000 & 1.478520000 & 2.287218000 \\
\hline & 6 & 0.717865000 & 0.396735000 & -0.219767000 \\
\hline & 6 & -0.759634000 & 0.211749000 & -0.503030000 \\
\hline & 6 & 2.211439000 & -1.262505000 & 0.774612000 \\
\hline & 6 & 2.032801000 & -2.766212000 & 0.479484000 \\
\hline & 6 & 2.856287000 & -0.470429000 & -0.394674000 \\
\hline
\end{tabular}




\begin{tabular}{|c|c|c|c|c|}
\hline & 1 & 2.807931000 & -1.132377000 & 1.684578000 \\
\hline & 6 & 3.299201000 & -3.391636000 & -0.120292000 \\
\hline & 1 & 1.208936000 & -2.895132000 & -0.233929000 \\
\hline & 1 & 1.742045000 & -3.284210000 & 1.398101000 \\
\hline & 6 & 3.960441000 & -1.201365000 & -1.166936000 \\
\hline & 6 & 3.679485000 & -2.686372000 & -1.427892000 \\
\hline & 1 & 3.125060000 & -4.460948000 & -0.293906000 \\
\hline & 1 & 4.133487000 & -3.321293000 & 0.594312000 \\
\hline & 1 & 4.150149000 & -0.662486000 & -2.104973000 \\
\hline & 1 & 4.882694000 & -1.117008000 & -0.575112000 \\
\hline & 1 & 4.567901000 & -3.150071000 & -1.875183000 \\
\hline & 1 & 2.861968000 & -2.798422000 & -2.153058000 \\
\hline & 1 & 3.294246000 & 0.449981000 & 0.024570000 \\
\hline & 7 & 1.665032000 & -0.133385000 & -1.214590000 \\
\hline & 1 & 1.899803000 & 0.571936000 & -1.912094000 \\
\hline \multirow{29}{*}{ 4-anti } & 6 & -3.452747000 & 0.138196000 & -0.597812000 \\
\hline & 6 & -2.909244000 & -0.859899000 & 0.255951000 \\
\hline & 6 & -3.780993000 & -1.749015000 & 0.910138000 \\
\hline & 6 & -5.152731000 & -1.631487000 & 0.704033000 \\
\hline & 6 & -5.673997000 & -0.639203000 & -0.148040000 \\
\hline & 6 & -4.830202000 & 0.253514000 & -0.808824000 \\
\hline & 6 & -0.348613000 & -1.478957000 & 0.902417000 \\
\hline & 6 & -1.473516000 & -0.756494000 & 0.282218000 \\
\hline & 1 & -3.379908000 & -2.516826000 & 1.564694000 \\
\hline & 1 & -5.831384000 & -2.315339000 & 1.206299000 \\
\hline & 1 & -6.747950000 & -0.565040000 & -0.294638000 \\
\hline & 1 & -5.235463000 & 1.017788000 & -1.465634000 \\
\hline & 16 & -2.189999000 & 1.156400000 & -1.324978000 \\
\hline & 8 & -0.372595000 & -2.535981000 & 1.534060000 \\
\hline & 7 & 0.813577000 & -0.776102000 & 0.584232000 \\
\hline & 6 & 0.947972000 & 2.833881000 & -0.654000000 \\
\hline & 6 & 1.284173000 & 4.099005000 & -0.166060000 \\
\hline & 6 & 1.603696000 & 4.268583000 & 1.185287000 \\
\hline & 6 & 1.581336000 & 3.164045000 & 2.040826000 \\
\hline & 6 & 1.245301000 & 1.897417000 & 1.550515000 \\
\hline & 6 & 0.930965000 & 1.717891000 & 0.197739000 \\
\hline & 1 & 0.695388000 & 2.731859000 & -1.707449000 \\
\hline & 1 & 1.293679000 & 4.950122000 & -0.841784000 \\
\hline & 1 & 1.866876000 & 5.251919000 & 1.565810000 \\
\hline & 1 & 1.829074000 & 3.283049000 & 3.092525000 \\
\hline & 1 & 1.232994000 & 1.042342000 & 2.218164000 \\
\hline & 6 & 0.533292000 & 0.338121000 & -0.369312000 \\
\hline & 6 & -0.971290000 & 0.235497000 & -0.506176000 \\
\hline & 6 & 2.013665000 & -1.517149000 & 0.133091000 \\
\hline
\end{tabular}




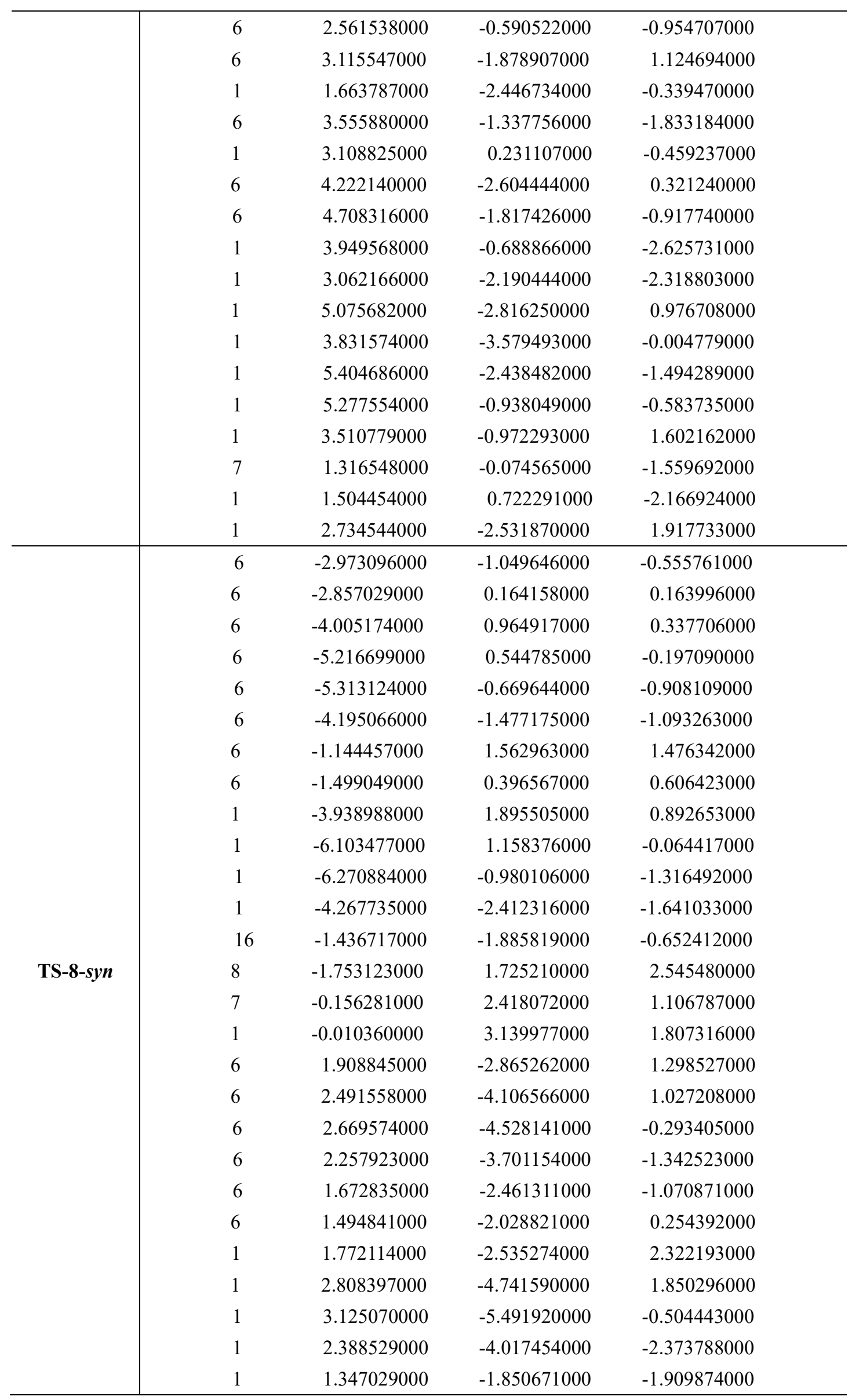




\begin{tabular}{|c|c|c|c|c|}
\hline & 6 & 0.842642000 & -0.673922000 & 0.605157000 \\
\hline & 8 & 0.985226000 & -0.344162000 & 1.929585000 \\
\hline & 6 & -0.635409000 & -0.605120000 & 0.246921000 \\
\hline & 6 & 0.494354000 & 2.707647000 & -0.183912000 \\
\hline & 6 & 1.684201000 & 3.642581000 & 0.116009000 \\
\hline & 6 & 0.895608000 & 1.478398000 & -1.029503000 \\
\hline & 1 & -0.214769000 & 3.258091000 & -0.819564000 \\
\hline & 6 & 2.449953000 & 4.052217000 & -1.148093000 \\
\hline & 1 & 2.365731000 & 3.142737000 & 0.815481000 \\
\hline & 1 & 1.301114000 & 4.534750000 & 0.627662000 \\
\hline & 6 & 1.713522000 & 1.920823000 & -2.261588000 \\
\hline & 6 & 2.912369000 & 2.818201000 & -1.932592000 \\
\hline & 1 & 3.306885000 & 4.675178000 & -0.866386000 \\
\hline & 1 & 1.804537000 & 4.670778000 & -1.788140000 \\
\hline & 1 & 2.022715000 & 1.032261000 & -2.826164000 \\
\hline & 1 & 1.024714000 & 2.468558000 & -2.917796000 \\
\hline & 1 & 3.405643000 & 3.117218000 & -2.864932000 \\
\hline & 1 & 3.659805000 & 2.263380000 & -1.347984000 \\
\hline & 8 & 3.184802000 & 0.749382000 & 1.797084000 \\
\hline & 1 & 3.226689000 & 1.578332000 & 2.298629000 \\
\hline & 1 & 1.970004000 & 0.091841000 & 2.067032000 \\
\hline & 1 & -0.005929000 & 0.991946000 & -1.400933000 \\
\hline & 7 & 1.648867000 & 0.425122000 & -0.254896000 \\
\hline & 1 & 2.222973000 & -0.090658000 & -0.925032000 \\
\hline & 1 & 2.369593000 & 0.809406000 & 0.488064000 \\
\hline \multirow{19}{*}{ TS-8-anti } & 6 & -2.063315000 & -2.210866000 & -0.693803000 \\
\hline & 6 & -2.499251000 & -1.239095000 & 0.238531000 \\
\hline & 6 & -3.869701000 & -1.169932000 & 0.564322000 \\
\hline & 6 & -4.756620000 & -2.056747000 & -0.033585000 \\
\hline & 6 & -4.303833000 & -3.020114000 & -0.959157000 \\
\hline & 6 & -2.956664000 & -3.106297000 & -1.298068000 \\
\hline & 6 & -1.600165000 & 0.678121000 & 1.698688000 \\
\hline & 6 & -1.403844000 & -0.421401000 & 0.707903000 \\
\hline & 1 & -4.221235000 & -0.433866000 & 1.280947000 \\
\hline & 1 & -5.813151000 & -2.009530000 & 0.215266000 \\
\hline & 1 & -5.014221000 & -3.704983000 & -1.413914000 \\
\hline & 1 & -2.608207000 & -3.847810000 & -2.011236000 \\
\hline & 16 & -0.332029000 & -2.126489000 & -0.942081000 \\
\hline & 8 & -2.081166000 & 0.460876000 & 2.820640000 \\
\hline & 7 & -1.264686000 & 1.921375000 & 1.285440000 \\
\hline & 1 & -1.376726000 & 2.643542000 & 1.991520000 \\
\hline & 6 & 2.720279000 & -1.302918000 & -1.231743000 \\
\hline & 6 & 3.743170000 & -2.199434000 & -1.556495000 \\
\hline & 6 & 4.367882000 & -2.945777000 & -0.554458000 \\
\hline
\end{tabular}




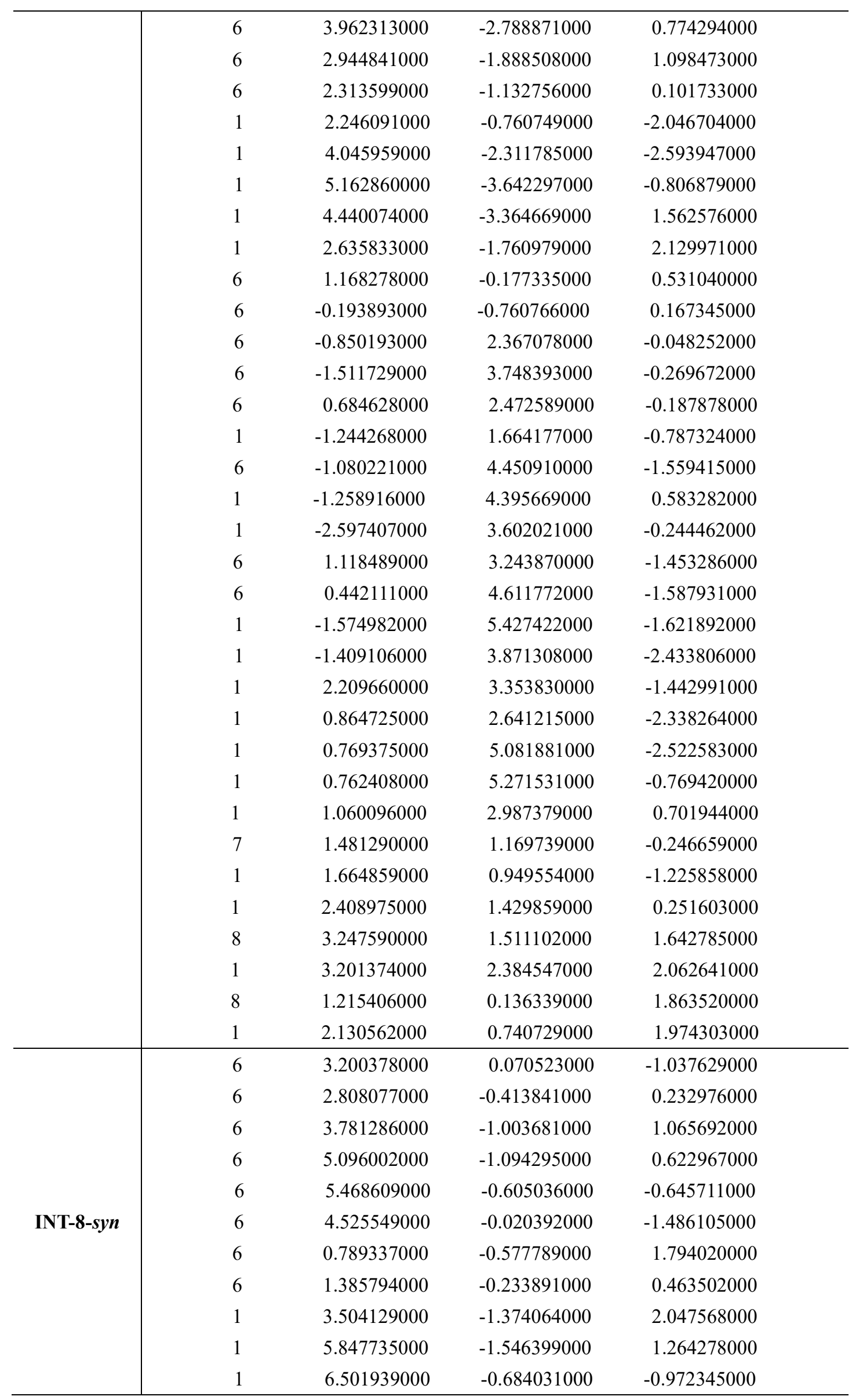




\begin{tabular}{|c|c|c|c|c|}
\hline & 1 & 4.809601000 & 0.355551000 & -2.465036000 \\
\hline & 16 & 1.840552000 & 0.745875000 & -1.910667000 \\
\hline & 8 & 1.291343000 & -0.087585000 & 2.819700000 \\
\hline & 7 & -0.286095000 & -1.401991000 & 1.887492000 \\
\hline & 1 & -0.567291000 & -1.530802000 & 2.855944000 \\
\hline & 6 & -2.400123000 & 2.525362000 & 0.052610000 \\
\hline & 6 & -2.749026000 & 3.689043000 & 0.741108000 \\
\hline & 6 & -1.780901000 & 4.408298000 & 1.449707000 \\
\hline & 6 & -0.462055000 & 3.950924000 & 1.459597000 \\
\hline & 6 & -0.113621000 & 2.781876000 & 0.773616000 \\
\hline & 6 & -1.076197000 & 2.049457000 & 0.065506000 \\
\hline & 1 & -3.167699000 & 1.998873000 & -0.504284000 \\
\hline & 1 & -3.779221000 & 4.035123000 & 0.718468000 \\
\hline & 1 & -2.052501000 & 5.314938000 & 1.983947000 \\
\hline & 1 & 0.303614000 & 4.499394000 & 2.002132000 \\
\hline & 1 & 0.917507000 & 2.447238000 & 0.799618000 \\
\hline & 6 & -0.722904000 & 0.776272000 & -0.738547000 \\
\hline & 8 & -0.859210000 & 1.034955000 & -2.143258000 \\
\hline & 6 & 0.746783000 & 0.375931000 & -0.584288000 \\
\hline & 6 & -0.945368000 & -2.319496000 & 0.936133000 \\
\hline & 6 & -2.256803000 & -2.765963000 & 1.619146000 \\
\hline & 6 & -1.188792000 & -1.698119000 & -0.467319000 \\
\hline & 1 & -0.311910000 & -3.205537000 & 0.790609000 \\
\hline & 6 & -3.111722000 & -3.679657000 & 0.734053000 \\
\hline & 1 & -2.841956000 & -1.874976000 & 1.890832000 \\
\hline & 1 & -2.007397000 & -3.273341000 & 2.560251000 \\
\hline & 6 & -2.114427000 & -2.606431000 & -1.304482000 \\
\hline & 6 & -3.420481000 & -2.998002000 & -0.604404000 \\
\hline & 1 & -4.036589000 & -3.935958000 & 1.264618000 \\
\hline & 1 & -2.578676000 & -4.623935000 & 0.550808000 \\
\hline & 1 & -2.301418000 & -2.113540000 & -2.265585000 \\
\hline & 1 & -1.553689000 & -3.523385000 & -1.537220000 \\
\hline & 1 & -4.002610000 & -3.665252000 & -1.251857000 \\
\hline & 1 & -4.043933000 & -2.109314000 & -0.427362000 \\
\hline & 8 & -3.664844000 & 0.542374000 & -2.291232000 \\
\hline & 1 & -1.815712000 & 1.077593000 & -2.360713000 \\
\hline & 1 & -3.958245000 & -0.076933000 & -2.978795000 \\
\hline & 1 & -0.238264000 & -1.673485000 & -1.001029000 \\
\hline & 7 & -1.671120000 & -0.300612000 & -0.361645000 \\
\hline & 1 & -3.248511000 & -0.004893000 & -1.583705000 \\
\hline & 1 & -2.021912000 & -0.113142000 & 0.574043000 \\
\hline \multirow{3}{*}{ INT-8-anti } & 6 & 3.274383000 & -0.266244000 & 0.980664000 \\
\hline & 6 & 2.845696000 & 0.113808000 & -0.313507000 \\
\hline & 6 & 3.796135000 & 0.610616000 & -1.228481000 \\
\hline
\end{tabular}




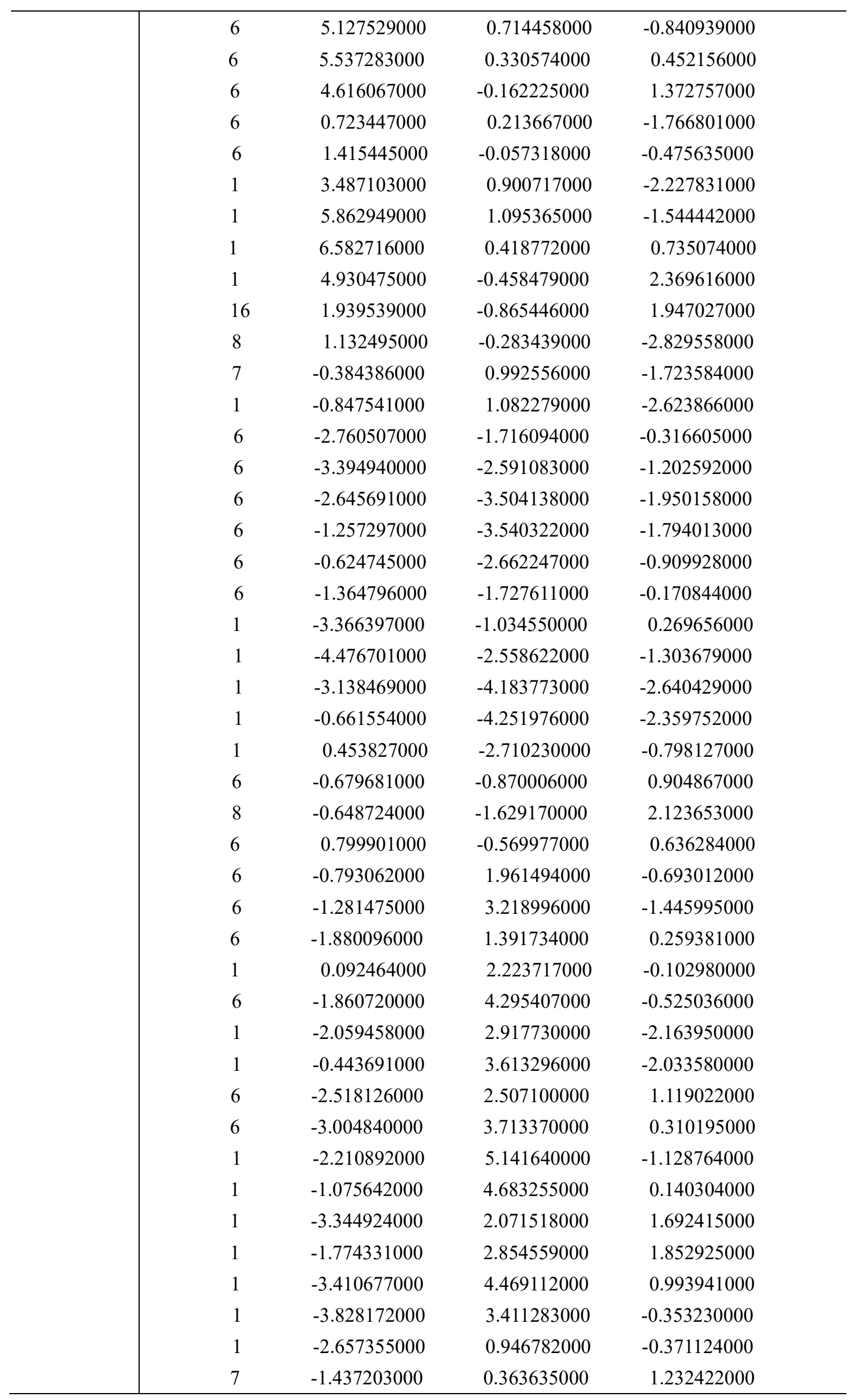




\begin{tabular}{|c|c|c|c|c|}
\hline & 1 & -0.899651000 & 0.825935000 & 1.967027000 \\
\hline & 1 & -2.878352000 & -0.247659000 & 2.468595000 \\
\hline & 8 & -3.261799000 & -0.962529000 & 3.031785000 \\
\hline & 1 & -1.561041000 & -1.661011000 & 2.490463000 \\
\hline & 1 & -3.251426000 & -0.618540000 & 3.939716000 \\
\hline \multirow{39}{*}{ TS-9-syn } & 6 & -4.392382000 & 0.642780000 & -0.801099000 \\
\hline & 6 & -3.790720000 & -0.595177000 & -1.136851000 \\
\hline & 6 & -4.570309000 & -1.577043000 & -1.785251000 \\
\hline & 6 & -5.901411000 & -1.309097000 & -2.073218000 \\
\hline & 6 & -6.482007000 & -0.069174000 & -1.731384000 \\
\hline & 6 & -5.735603000 & 0.916982000 & -1.095471000 \\
\hline & 6 & -1.543972000 & -1.825307000 & -1.103829000 \\
\hline & 6 & -2.409551000 & -0.659818000 & -0.712940000 \\
\hline & 1 & -4.125498000 & -2.528059000 & -2.059188000 \\
\hline & 1 & -6.505304000 & -2.062878000 & -2.570454000 \\
\hline & 1 & -7.525151000 & 0.119905000 & -1.968717000 \\
\hline & 1 & -6.181096000 & 1.872038000 & -0.833620000 \\
\hline & 16 & -3.257068000 & 1.720272000 & -0.019544000 \\
\hline & 8 & -1.493284000 & -2.118650000 & -2.310361000 \\
\hline & 7 & -0.856656000 & -2.550918000 & -0.193953000 \\
\hline & 1 & -0.327297000 & -3.291288000 & -0.646581000 \\
\hline & 6 & -0.211109000 & 3.043134000 & -0.403869000 \\
\hline & 6 & 0.195788000 & 4.367897000 & -0.264851000 \\
\hline & 6 & 0.589443000 & 4.857096000 & 0.987078000 \\
\hline & 6 & 0.558789000 & 4.014113000 & 2.099133000 \\
\hline & 6 & 0.145134000 & 2.685548000 & 1.962973000 \\
\hline & 6 & -0.229719000 & 2.179826000 & 0.707175000 \\
\hline & 1 & -0.503280000 & 2.675664000 & -1.381860000 \\
\hline & 1 & 0.208999000 & 5.018494000 & -1.134617000 \\
\hline & 1 & 0.908724000 & 5.890145000 & 1.092762000 \\
\hline & 1 & 0.843599000 & 4.387578000 & 3.078522000 \\
\hline & 1 & 0.085964000 & 2.062179000 & 2.849381000 \\
\hline & 6 & -0.703164000 & 0.763153000 & 0.575102000 \\
\hline & 8 & 0.562610000 & 0.192459000 & -0.941912000 \\
\hline & 6 & -1.989033000 & 0.499003000 & -0.109078000 \\
\hline & 6 & -0.772989000 & -2.585746000 & 1.274836000 \\
\hline & 6 & 0.635373000 & -3.076035000 & 1.659954000 \\
\hline & 6 & -1.139642000 & -1.271017000 & 1.993929000 \\
\hline & 1 & -1.502893000 & -3.324546000 & 1.638034000 \\
\hline & 6 & 0.779919000 & -3.251209000 & 3.178285000 \\
\hline & 1 & 1.379535000 & -2.360010000 & 1.285975000 \\
\hline & 1 & 0.832598000 & -4.029736000 & 1.153774000 \\
\hline & 6 & -0.959591000 & -1.446094000 & 3.517657000 \\
\hline & 6 & 0.432565000 & -1.954351000 & 3.921550000 \\
\hline
\end{tabular}




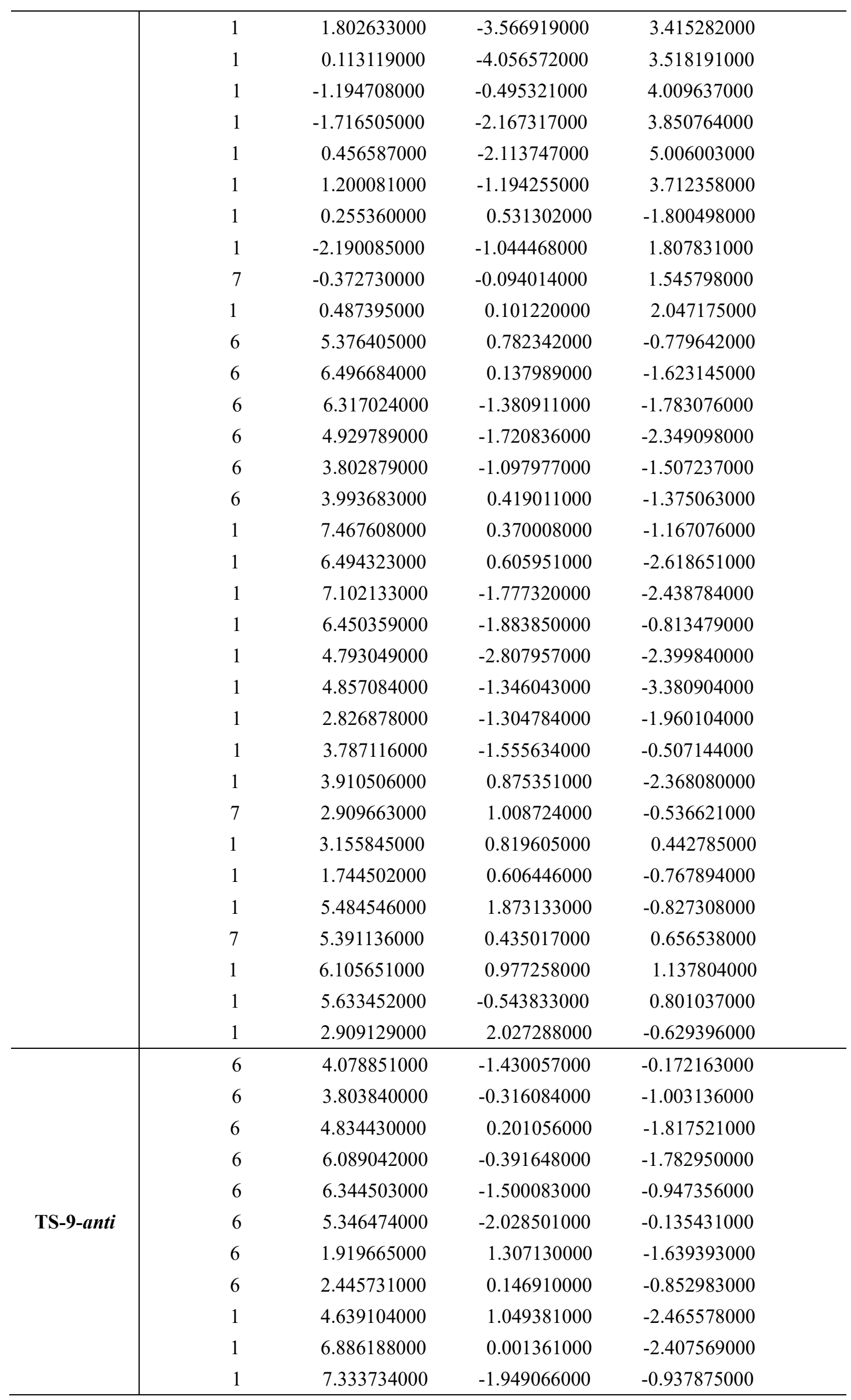




\begin{tabular}{|c|c|c|c|c|c|}
\hline & & 1 & 5.543735000 & -2.881769000 & 0.506706000 \\
\hline & & 16 & 2.665834000 & -1.917326000 & 0.733092000 \\
\hline & & 8 & 1.980690000 & 1.322225000 & -2.876278000 \\
\hline & & 7 & 1.429721000 & 2.336988000 & -0.914850000 \\
\hline & & 1 & 1.055969000 & 3.110516000 & -1.457091000 \\
\hline & & 6 & -0.446232000 & -2.885825000 & 0.033996000 \\
\hline & & 6 & -1.027599000 & -4.075552000 & 0.466487000 \\
\hline & & 6 & -1.532138000 & -4.184421000 & 1.768526000 \\
\hline & & 6 & -1.440146000 & -3.096731000 & 2.637754000 \\
\hline & & 6 & -0.856955000 & -1.900929000 & 2.206497000 \\
\hline & & 6 & -0.366925000 & -1.777740000 & 0.895012000 \\
\hline & & 1 & -0.071484000 & -2.806146000 & -0.979263000 \\
\hline & & 1 & -1.091918000 & -4.918644000 & -0.215637000 \\
\hline & & 1 & -1.986241000 & -5.113559000 & 2.101764000 \\
\hline & & 1 & -1.810605000 & -3.173885000 & 3.656008000 \\
\hline & & 1 & -0.757174000 & -1.080113000 & 2.910355000 \\
\hline & & 6 & 0.296661000 & -0.492627000 & 0.470784000 \\
\hline & & 8 & -0.538470000 & -0.331902000 & -1.469826000 \\
\hline & & 6 & 1.715277000 & -0.597310000 & 0.038813000 \\
\hline & & 6 & 1.474597000 & 2.488738000 & 0.539906000 \\
\hline & & 6 & 1.728472000 & 3.974192000 & 0.871896000 \\
\hline & & 6 & 0.178599000 & 2.018286000 & 1.221949000 \\
\hline & & 1 & 2.319639000 & 1.907748000 & 0.917710000 \\
\hline & & 6 & 1.774765000 & 4.239781000 & 2.381640000 \\
\hline & & 1 & 0.932870000 & 4.583609000 & 0.418138000 \\
\hline & & 1 & 2.669780000 & 4.270375000 & 0.395217000 \\
\hline & & 6 & 0.223002000 & 2.279405000 & 2.743698000 \\
\hline & & 6 & 0.488147000 & 3.756919000 & 3.061323000 \\
\hline & & 1 & 1.924852000 & 5.311406000 & 2.558410000 \\
\hline & & 1 & 2.639713000 & 3.721659000 & 2.819870000 \\
\hline & & 1 & -0.726059000 & 1.961338000 & 3.191874000 \\
\hline & & 1 & 1.010692000 & 1.652271000 & 3.183055000 \\
\hline & & 1 & 0.552946000 & 3.888395000 & 4.147866000 \\
\hline & & 1 & -0.361676000 & 4.364335000 & 2.718382000 \\
\hline & & 1 & -0.645263000 & 2.599608000 & 0.786965000 \\
\hline & & 7 & -0.216802000 & 0.599731000 & 1.025999000 \\
\hline & & 1 & -1.169524000 & 0.459563000 & 1.350622000 \\
\hline & & 1 & -0.014496000 & -0.776380000 & -2.157430000 \\
\hline & & 6 & -3.910812000 & 0.079567000 & -1.152817000 \\
\hline & & 6 & -4.234632000 & -1.144112000 & -0.290714000 \\
\hline & & 6 & -5.239033000 & -0.788140000 & 0.816816000 \\
\hline & & 6 & -6.511762000 & -0.152715000 & 0.236316000 \\
\hline & & 6 & -6.177873000 & 1.065819000 & -0.640173000 \\
\hline & & 6 & -5.177118000 & 0.716939000 & -1.757762000 \\
\hline
\end{tabular}




\begin{tabular}{|c|c|c|c|c|}
\hline & 1 & -3.405650000 & 0.836630000 & -0.537246000 \\
\hline & 1 & -3.312648000 & -1.550336000 & 0.140327000 \\
\hline & 1 & -4.663288000 & -1.928443000 & -0.932946000 \\
\hline & 1 & -5.488077000 & -1.690483000 & 1.388344000 \\
\hline & 1 & -4.769286000 & -0.087844000 & 1.522871000 \\
\hline & 1 & -7.191420000 & 0.143777000 & 1.044941000 \\
\hline & 1 & -7.047937000 & -0.899287000 & -0.367822000 \\
\hline & 1 & -7.088969000 & 1.477658000 & -1.092587000 \\
\hline & 1 & -5.745440000 & 1.862539000 & -0.015402000 \\
\hline & 1 & -5.639762000 & -0.027087000 & -2.422077000 \\
\hline & 7 & -2.943263000 & -0.268843000 & -2.235430000 \\
\hline & 1 & -1.803286000 & -0.348393000 & -1.855569000 \\
\hline & 1 & -3.002491000 & 0.473169000 & -2.943932000 \\
\hline & 7 & -4.773350000 & 1.845755000 & -2.613955000 \\
\hline & 1 & -4.552380000 & 2.662171000 & -2.042274000 \\
\hline & 1 & -5.543441000 & 2.122027000 & -3.220770000 \\
\hline & 1 & -3.210942000 & -1.142498000 & -2.695441000 \\
\hline \multirow{27}{*}{ INT-9-syn } & 6 & 2.974093000 & 0.643867000 & -0.764474000 \\
\hline & 6 & 2.686466000 & -0.432162000 & 0.115095000 \\
\hline & 6 & 3.736573000 & -1.293153000 & 0.507260000 \\
\hline & 6 & 5.012241000 & -1.074491000 & 0.012348000 \\
\hline & 6 & 5.276103000 & -0.000981000 & -0.867449000 \\
\hline & 6 & 4.265361000 & 0.866136000 & -1.263832000 \\
\hline & 6 & 0.796742000 & -1.384814000 & 1.566994000 \\
\hline & 6 & 1.288655000 & -0.492901000 & 0.457139000 \\
\hline & 1 & 3.538974000 & -2.113723000 & 1.188406000 \\
\hline & 1 & 5.822496000 & -1.736103000 & 0.304190000 \\
\hline & 1 & 6.285301000 & 0.151819000 & -1.238958000 \\
\hline & 1 & 4.469652000 & 1.692144000 & -1.937900000 \\
\hline & 16 & 1.555589000 & 1.603887000 & -1.106482000 \\
\hline & 8 & 1.431834000 & -1.360965000 & 2.630446000 \\
\hline & 7 & -0.333427000 & -2.127279000 & 1.454833000 \\
\hline & 1 & -0.521094000 & -2.623923000 & 2.322449000 \\
\hline & 6 & -2.635195000 & 2.484555000 & -0.493097000 \\
\hline & 6 & -3.165772000 & 3.746190000 & -0.240328000 \\
\hline & 6 & -2.519291000 & 4.614146000 & 0.648272000 \\
\hline & 6 & -1.332120000 & 4.224240000 & 1.277617000 \\
\hline & 6 & -0.785005000 & 2.969628000 & 1.019582000 \\
\hline & 6 & -1.439046000 & 2.083482000 & 0.138945000 \\
\hline & 1 & -3.121574000 & 1.836940000 & -1.216955000 \\
\hline & 1 & -4.075037000 & 4.058128000 & -0.744818000 \\
\hline & 1 & -2.937601000 & 5.597416000 & 0.843172000 \\
\hline & 1 & -0.833112000 & 4.897016000 & 1.968239000 \\
\hline & 1 & 0.128512000 & 2.662701000 & 1.518587000 \\
\hline
\end{tabular}




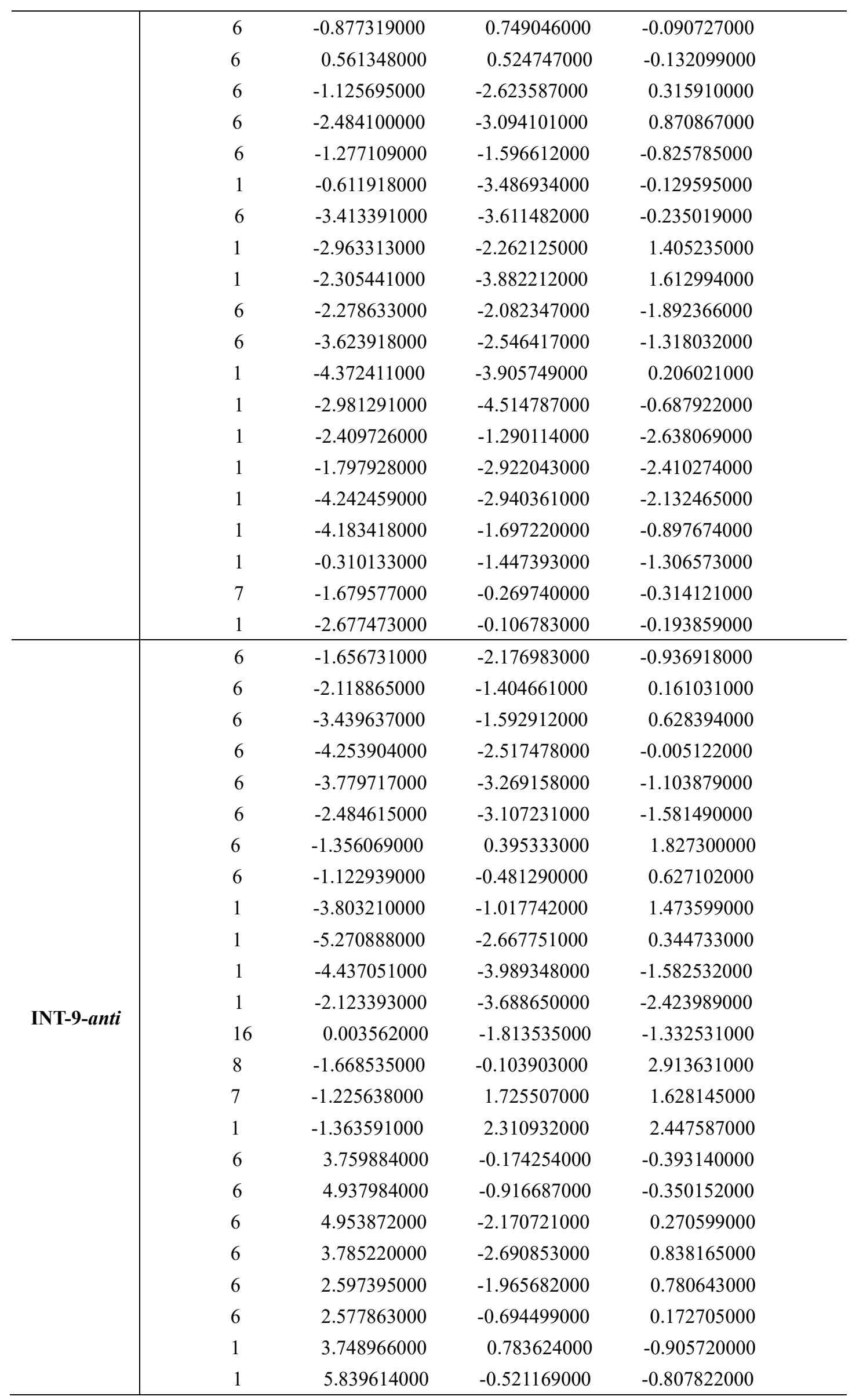




\begin{tabular}{|c|c|c|c|c|}
\hline & 1 & 5.875210000 & -2.744900000 & 0.306762000 \\
\hline & 1 & 3.799303000 & -3.661126000 & 1.325132000 \\
\hline & 1 & 1.695533000 & -2.365532000 & 1.232579000 \\
\hline & 6 & 1.333351000 & 0.100905000 & 0.168429000 \\
\hline & 6 & 0.063065000 & -0.573264000 & -0.075730000 \\
\hline & 6 & -0.912912000 & 2.371706000 & 0.355113000 \\
\hline & 6 & -1.635606000 & 3.725348000 & 0.233640000 \\
\hline & 6 & 0.599150000 & 2.590995000 & 0.189345000 \\
\hline & 1 & -1.278868000 & 1.727384000 & -0.447256000 \\
\hline & 6 & -1.331176000 & 4.393945000 & -1.115591000 \\
\hline & 1 & -1.321676000 & 4.382907000 & 1.056893000 \\
\hline & 1 & -2.711739000 & 3.554002000 & 0.347660000 \\
\hline & 6 & 0.923363000 & 3.218837000 & -1.182056000 \\
\hline & 6 & 0.179036000 & 4.555417000 & -1.338529000 \\
\hline & 1 & -1.826012000 & 5.371087000 & -1.157193000 \\
\hline & 1 & -1.760371000 & 3.787154000 & -1.925275000 \\
\hline & 1 & 2.004444000 & 3.372079000 & -1.271884000 \\
\hline & 1 & 0.625714000 & 2.519618000 & -1.974582000 \\
\hline & 1 & 0.376411000 & 4.959859000 & -2.337978000 \\
\hline & 1 & 0.583205000 & 5.280023000 & -0.618017000 \\
\hline & 1 & 0.926589000 & 3.276804000 & 0.978448000 \\
\hline & 7 & 1.455652000 & 1.390877000 & 0.406184000 \\
\hline & 1 & 2.394923000 & 1.662960000 & 0.690849000 \\
\hline \multirow{21}{*}{$5-s y n$} & 6 & -2.980054000 & -0.550962000 & -0.838373000 \\
\hline & 6 & -2.689625000 & 0.382221000 & 0.186674000 \\
\hline & 6 & -3.744588000 & 1.149557000 & 0.721965000 \\
\hline & 6 & -5.033668000 & 0.976685000 & 0.231727000 \\
\hline & 6 & -5.301403000 & 0.042885000 & -0.789722000 \\
\hline & 6 & -4.278547000 & -0.728896000 & -1.333629000 \\
\hline & 6 & -0.735333000 & 1.200395000 & 1.657831000 \\
\hline & 6 & -1.274448000 & 0.412416000 & 0.504415000 \\
\hline & 1 & -3.545184000 & 1.862814000 & 1.514722000 \\
\hline & 1 & -5.847383000 & 1.568017000 & 0.642507000 \\
\hline & 1 & -6.316636000 & -0.078764000 & -1.157214000 \\
\hline & 1 & -4.482029000 & -1.449150000 & -2.120685000 \\
\hline & 16 & -1.536307000 & -1.402782000 & -1.356804000 \\
\hline & 8 & -1.340710000 & 1.150963000 & 2.743266000 \\
\hline & 7 & 0.428313000 & 1.896727000 & 1.556726000 \\
\hline & 1 & 0.662442000 & 2.321957000 & 2.450482000 \\
\hline & 6 & 2.738599000 & -2.418718000 & -0.428324000 \\
\hline & 6 & 3.263679000 & -3.675254000 & -0.128481000 \\
\hline & 6 & 2.540450000 & -4.568029000 & 0.672904000 \\
\hline & 6 & 1.287452000 & -4.196001000 & 1.168193000 \\
\hline & 6 & 0.756327000 & -2.940476000 & 0.860794000 \\
\hline
\end{tabular}




\begin{tabular}{|c|c|c|c|c|}
\hline & 6 & 1.478048000 & -2.035293000 & 0.064135000 \\
\hline & 1 & 3.294012000 & -1.724101000 & -1.050361000 \\
\hline & 1 & 4.234974000 & -3.962002000 & -0.523070000 \\
\hline & 1 & 2.950821000 & -5.547003000 & 0.906243000 \\
\hline & 1 & 0.721854000 & -4.880974000 & 1.794185000 \\
\hline & 1 & -0.215113000 & -2.658301000 & 1.256470000 \\
\hline & 6 & 0.937742000 & -0.676633000 & -0.231899000 \\
\hline & 6 & -0.542158000 & -0.484582000 & -0.231573000 \\
\hline & 6 & 1.166516000 & 2.497302000 & 0.424184000 \\
\hline & 6 & 2.563064000 & 2.884276000 & 0.945489000 \\
\hline & 6 & 1.237309000 & 1.603033000 & -0.838005000 \\
\hline & 1 & 0.644189000 & 3.416620000 & 0.119578000 \\
\hline & 6 & 3.413310000 & 3.553689000 & -0.142517000 \\
\hline & 1 & 3.068160000 & 1.980942000 & 1.310393000 \\
\hline & 1 & 2.449483000 & 3.561563000 & 1.802786000 \\
\hline & 6 & 2.138388000 & 2.266561000 & -1.901385000 \\
\hline & 6 & 3.527663000 & 2.654108000 & -1.380279000 \\
\hline & 1 & 4.406987000 & 3.783757000 & 0.261292000 \\
\hline & 1 & 2.957967000 & 4.514402000 & -0.425593000 \\
\hline & 1 & 2.218418000 & 1.583075000 & -2.755501000 \\
\hline & 1 & 1.620103000 & 3.165122000 & -2.265629000 \\
\hline & 1 & 4.089309000 & 3.164737000 & -2.172888000 \\
\hline & 1 & 4.090931000 & 1.747529000 & -1.122954000 \\
\hline & 1 & 0.226755000 & 1.538030000 & -1.260049000 \\
\hline & 7 & 1.752289000 & 0.280248000 & -0.500544000 \\
\hline \multirow{19}{*}{ 5-anti } & 6 & 1.443555000 & 2.265289000 & -0.996675000 \\
\hline & 6 & 1.901719000 & 1.643513000 & 0.190976000 \\
\hline & 6 & 3.141045000 & 2.039598000 & 0.734404000 \\
\hline & 6 & 3.882098000 & 3.025933000 & 0.094327000 \\
\hline & 6 & 3.411115000 & 3.631534000 & -1.088636000 \\
\hline & 6 & 2.191207000 & 3.256838000 & -1.645490000 \\
\hline & 6 & 1.239034000 & -0.194004000 & 1.878576000 \\
\hline & 6 & 0.981441000 & 0.628836000 & 0.655657000 \\
\hline & 1 & 3.504274000 & 1.577865000 & 1.647330000 \\
\hline & 1 & 4.836889000 & 3.335290000 & 0.510416000 \\
\hline & 1 & 4.004949000 & 4.402150000 & -1.572380000 \\
\hline & 1 & 1.828059000 & 3.723886000 & -2.556282000 \\
\hline & 16 & -0.115094000 & 1.630554000 & -1.488448000 \\
\hline & 8 & 1.463170000 & 0.331660000 & 2.980748000 \\
\hline & 7 & 1.234149000 & -1.533678000 & 1.692984000 \\
\hline & 1 & 1.382467000 & -2.090268000 & 2.530182000 \\
\hline & 6 & -3.821605000 & -0.192484000 & -0.388383000 \\
\hline & 6 & -5.045352000 & 0.472839000 & -0.300922000 \\
\hline & 6 & -5.123069000 & 1.724569000 & 0.321426000 \\
\hline
\end{tabular}




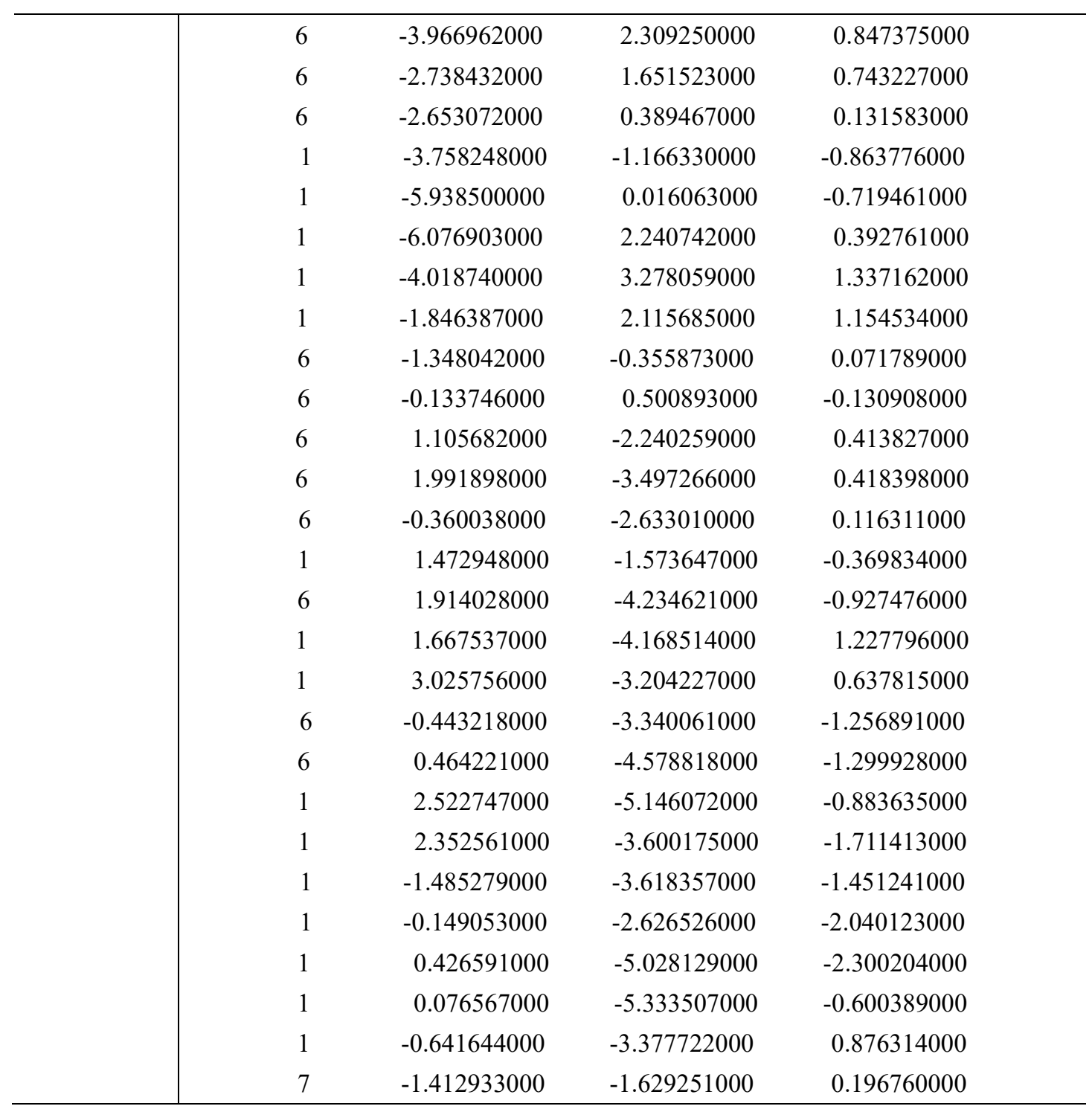

Table S3. Electronic energies $\left(\boldsymbol{E}^{\boldsymbol{B} 3}\right.$ in a.u.), entropies $\left(\boldsymbol{S}^{\boldsymbol{B} 3}\right.$ in cal $\left.\mathrm{mol}^{-1} \mathrm{~K}^{-1}\right)$, and Gibbs free energies $\left(\boldsymbol{G}^{\boldsymbol{B} 3}\right.$ in a.u, computed at the B3LYP-PCM/6-31+G* level of theory in DMF, and single-point energies $\left(\boldsymbol{E}^{\boldsymbol{m} 06}\right.$ in a.u.) computed at the $\mathrm{m} 06-\mathrm{PCM} / 6-311++\mathrm{G}(3 \mathrm{df}, 2 \mathrm{p})$ level of theory in DMF.

\begin{tabular}{|c|c|c|c|c|}
\hline Species & $\boldsymbol{E}^{\boldsymbol{B 3}}$ & $\boldsymbol{S}^{\boldsymbol{B 3}}$ & $\boldsymbol{G}^{\boldsymbol{B 3}}$ & $\boldsymbol{E}^{\boldsymbol{m 0 6}}$ \\
\hline 1 & -855.92066 & 104.704 & -855.87446 & -855.7502479 \\
\hline $3 \mathrm{a}$ & -346.59435 & 101.482 & -346.44041 & -346.4430614 \\
\hline TS-1 & -1202.50293 & 162.028 & -1202.27049 & -1202.186701 \\
\hline INT-1 & -1202.50343 & 165.211 & -1202.2713 & -1202.186845 \\
\hline TS-2 & -1202.48737 & 161.095 & -1202.25711 & -1202.17001 \\
\hline INT-2 & -1202.5341 & 165.274 & -1202.30021 & -1202.213544 \\
\hline TS-3 & -4158.56846 & 233.089 & -4158.23133 & -4160.36795 \\
\hline 2 & -2956.04016 & 108.418 & -2955.9658 & -2958.161626 \\
\hline INT-3 & -4158.59901 & 239.313 & -4158.26395 & -4160.408907 \\
\hline INT-3-1 & -1586.23631 & 225.429 & -1585.91157 & -1585.756569 \\
\hline $3 \mathrm{aHBr}$ & -2918.97298 & 120.802 & -2918.81293 & -2921.11268 \\
\hline TS-4 & -1932.80745 & 291.83 & -1932.30694 & -1932.176377 \\
\hline
\end{tabular}




\begin{tabular}{|c|c|c|c|c|}
\hline INT-4 & -1585.74759 & 219.767 & -1585.43328 & -1585.271344 \\
\hline $3 \mathrm{aH}+$ & -347.05225 & 101.061 & -346.8821 & -346.8967883 \\
\hline TS-5 & -1585.74659 & 214.501 & -1585.43009 & -1585.269413 \\
\hline INT-5 & -1585.75777 & 215.574 & -1585.4403 & -1585.280715 \\
\hline INT-5-1 & -1586.23643 & 217.45 & -1585.90574 & -1585.764416 \\
\hline TS-6 & -1932.81014 & 272.465 & -1932.29783 & -1932.19403 \\
\hline INT-6 & -1585.76163 & 211.884 & -1585.44147 & -1585.288835 \\
\hline INT-6-1 & -1586.22629 & 217.428 & -1585.89567 & -1585.758601 \\
\hline TS-7 & -1586.19559 & 212.161 & -1585.86677 & -1585.719233 \\
\hline INT-7-anti & -1586.25648 & 225.45 & -1585.9318 & -1585.783568 \\
\hline INT-7-syn & -1586.25631 & 230.445 & -1585.93510 & -1585.782392 \\
\hline TS-10-syn & -1586.22402 & 220.813 & -1585.89718 & -1585.751048 \\
\hline INT-10-syn & -1586.24528 & 218.061 & -1585.91587 & -1585.782102 \\
\hline TS-11-syn & -1856.81100 & 266.145 & -1856.30729 & -1856.197191 \\
\hline INT-11-syn & -1433.77962 & 190.463 & -1433.47796 & -1433.313162 \\
\hline TS-12-syn & -1433.76966 & 184.243 & -1433.46553 & -1433.30536 \\
\hline INT-12-syn & -1433.79969 & 181.246 & -1433.48960 & -1433.335347 \\
\hline 4-syn & -1433.36196 & 181.926 & -1433.06763 & -1432.9010835 \\
\hline syn- $3 \mathrm{a} \cdot \mathrm{H}^{+}$ & -347.04957 & 100.474 & -346.87898 & -346.8950959 \\
\hline syn-3a & -346.59111 & 100.838 & -346.43644 & -346.4402926 \\
\hline TS-10-anti & -1586.22448 & 219.338 & -1585.89635 & -1585.749632 \\
\hline INT-10-anti & -1586.25162 & 220.211 & -1585.92413 & -1585.785272 \\
\hline TS-11-anti & -1856.80716 & 265.973 & -1856.30559 & -1856.192581 \\
\hline INT-11-anti & -1433.77933 & 192.549 & -1433.47934 & -1433.313242 \\
\hline TS-12-anti & -1433.77989 & 182.753 & -1433.4745 & -1433.314133 \\
\hline INT-12-anti & -1433.80166 & 182.231 & -1433.49228 & -1433.336719 \\
\hline 4-anti & -1433.36322 & 182.996 & -1433.06979 & -1432.901792 \\
\hline anti-3a & -346.59435 & 101.482 & -346.44041 & -346.4430614 \\
\hline anti-3a $\cdot \mathrm{H}^{+}$ & -347.05225 & 101.061 & -346.88210 & -346.8967883 \\
\hline TS-8-syn & -1586.20556 & 201.219 & -1585.86929 & -1585.737415 \\
\hline INT-8-syn & -1586.23036 & 210.233 & -1585.89492 & -1585.771828 \\
\hline TS-9-syn & -1856.82426 & 261.649 & -1856.31898 & -1856.209509 \\
\hline INT-9-syn & -1433.81214 & 186.567 & -1433.50665 & -1433.343852 \\
\hline 5-syn & -1433.36838 & 186.953 & -1433.07776 & -1432.900481 \\
\hline TS-8-anti & -1586.20795 & 203.059 & -1585.87268 & -1585.738584 \\
\hline INT-8-anti & -1586.23795 & 208.705 & -1585.90147 & -1585.775391 \\
\hline TS-9-anti & -1856.82904 & 264.015 & -1856.32535 & -1856.21171 \\
\hline INT-9-anti & -1433.81492 & 186.237 & -1433.50910 & -1433.345952 \\
\hline 5-anti & -1433.36757 & 186.537 & -1433.07678 & -1432.89927 \\
\hline
\end{tabular}


Table S4. The number of imaginary frequencies of the main stationary points, and the values of imaginary frequencies $\left(\mathrm{cm}^{-1}\right)$ for all the located transition states, determined at the B3LYP-PCM/6$31+\mathrm{G}^{*}$ level of theory in DMF.

\begin{tabular}{|c|c|c|c|c|c|}
\hline Names & No. of I.F. & value of I.F. & Names & No. of I.F. & value of I.F. \\
\hline TS-1 & 1 & $-82.5 \mathrm{i}$ & INT-11-syn & 0 & none \\
\hline INT-1 & 0 & none & TS-12-syn & 1 & $-44.8 \mathrm{i}$ \\
\hline TS-2 & 1 & $-1346.2 \mathrm{i}$ & INT-12-syn & 0 & none \\
\hline INT-2 & 0 & none & 4-syn & 0 & none \\
\hline TS-3 & 1 & $-404.7 \mathrm{i}$ & TS-10-anti & 1 & $-19.9 \mathrm{i}$ \\
\hline INT-3 & 0 & none & INT-10-anti & 0 & none \\
\hline INT-3-1 & 0 & none & TS-11-anti & 1 & $-408.7 \mathrm{i}$ \\
\hline TS-4 & 1 & $-1191.8 \mathrm{i}$ & INT-11-anti & 0 & none \\
\hline INT-4 & 0 & none & TS-12-anti & 1 & $-73.9 \mathrm{i}$ \\
\hline TS-5 & 1 & $-147.7 \mathrm{i}$ & INT-12-anti & 0 & none \\
\hline INT-5 & 0 & none & 4-anti & 0 & none \\
\hline INT-5-1 & 0 & none & TS-8-syn & 1 & $-261.4 \mathrm{i}$ \\
\hline TS-6 & 1 & $-1308.5 \mathrm{i}$ & INT-8-syn & 0 & none \\
\hline INT-6 & 0 & none & TS-9-syn & 1 & $-974.0 \mathrm{i}$ \\
\hline INT-6-1 & 0 & none & INT-9-syn & 0 & none \\
\hline TS-7 & 1 & $-555.9 \mathrm{i}$ & $\mathbf{5 - s y n}$ & 0 & none \\
\hline INT-7-anti & 0 & none & TS-8-anti & 1 & $-370.3 \mathrm{i}$ \\
\hline INT-7-syn & 0 & none & INT-8-anti & 0 & none \\
\hline TS-10-syn & 1 & $-124.7 \mathrm{i}$ & TS-9-anti & 1 & $-737.2 \mathrm{i}$ \\
\hline INT-10-syn & 0 & none & INT-9-anti & 0 & none \\
\hline TS-11-syn & 1 & $-160.0 \mathrm{i}$ & $\mathbf{5 - a n t i}$ & 0 & none \\
\hline
\end{tabular}

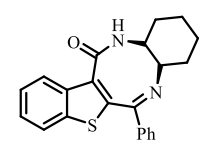

-10.0
syn-5

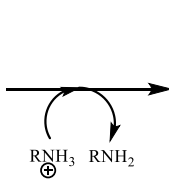

$\underset{\oplus}{\mathrm{RNH}_{3}} \mathrm{RNH}_{2}$

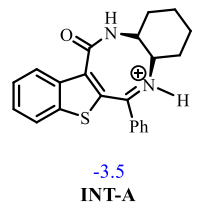

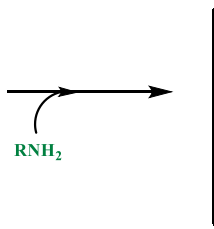

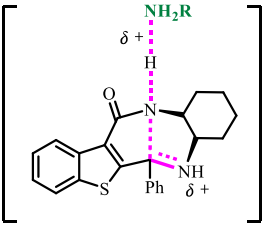

27.6

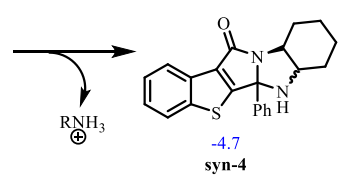

syn-4

Figure S1. The reaction mechanism and free-energy variations (in $\mathrm{kcal} / \mathrm{mol}$ ) for the conversion between product $\mathbf{5}$ and product $\mathbf{4}$ for 3a-syn, at the M06//B3LYP level of theory.

\section{Computational Methods}

All calculations were finished using the Gaussian 16 computational program $^{[1]}$. Geometrical optimizations were performed by the B3LYP density functional method ${ }^{[2]}$ with the $6-31+g^{*}$ basis set for all elements. The default self-consistent reaction field polarizable continuum model ${ }^{[3]}$ was used to consider the implicit solvation effects of DMF. All of the resultant stationary point geometries were characterized by vibrational 
analyses, from which zero point energies and Gibbs free-energies were obtained, in addition to confirming whether all of the structures resided at minima or first-order saddle points on the potential energy surfaces. To further refine the electronic energies obtained, single-point energies were computed using the m06 density functional method $^{[4]}$ with the $6-311++G(3 \mathrm{df}, 2 \mathrm{p})$ basis set for all elements. Considering the default entropic data obtained from the Gaussian output files are the idea-gas-phase entropies, which would exaggerate the activation entropies for the bimolecular reaction in solution. ${ }^{[5]}$ Hence, the default entropies are scaled by a factor of 0.7 in Gibbs freeenergy determinations.

\section{X-ray data collection and structure determinations}

Single crystals of $\mathbf{4 p}, \mathbf{5 a}, \mathbf{6}$ and $\mathbf{7}$ were grown by slow diffusion of $n$-hexane into a dichloromethane solution. X-ray single-crystal diffraction data was collected on a Rigaku XtaLAB P200 diffractometer at 293(2) K with MoK $\alpha$ radiation $(\lambda=0.71073 \AA)$ or CuKa $(\lambda=1.54184 \AA)$ in the $\omega$ scan mode. The program SAINT was used for integration of the diffraction profiles. All of the structures were solved using direct methods using the SHELXS program of the SHELXTL package and refined using full matrix least-squares methods with SHELXL (semi empirical absorption corrections were applied using the SADABS program). Other non-hydrogen atoms were located in successive difference Fourier syntheses and refined with anisotropic thermal parameters on $F 2$. The hydrogen atoms were generated theoretically onto the specific atoms and refined isotopically with fixed thermal factors. Detailed crystallographic data were summarized in Table S1 and S2. 
Table S5 Crystal Date and Structure Refinements for $\mathbf{4 p , 5 a}$

\begin{tabular}{|c|c|c|}
\hline & $4 p$ & $\mathbf{5 a}$ \\
\hline Chemical formula & $\mathrm{C}_{23} \mathrm{H}_{22} \mathrm{~N}_{2} \mathrm{OS}$ & $\mathrm{C}_{24} \mathrm{H}_{24} \mathrm{Cl}_{2} \mathrm{~N}_{2} \mathrm{OS}$ \\
\hline Formula weight & 374.48 & 459.41 \\
\hline Crystal system & triclinic & monoclinic \\
\hline Space group & $\mathrm{P}-1$ & P 1 20/c 1 \\
\hline$a(\AA)$ & $6.3337(11)$ & $9.9614(1)$ \\
\hline$b(\AA)$ & $11.4985(18)$ & $10.6273(1)$ \\
\hline$c(\AA)$ & $13.3558(15)$ & $21.4625(3)$ \\
\hline$V\left(\AA^{3}\right)$ & $949.0(3)$ & $2219.04(4)$ \\
\hline$\alpha\left(^{\circ}\right)$ & $90.378(11)$ & 90 \\
\hline$\beta\left({ }^{\circ}\right)$ & $102.026(12)$ & $102.405(1)$ \\
\hline$\gamma\left(\left(^{\circ}\right)\right.$ & $93.819(13)$ & 90 \\
\hline$Z$ & 2 & 4 \\
\hline $\mathrm{F}(000)$ & 396.0 & 960.0 \\
\hline$G O F$ & 1.081 & 1.085 \\
\hline$D / \mathrm{g} \mathrm{cm}^{-3}$ & 1.311 & 1.375 \\
\hline$\mu\left(\mathrm{mm}^{-1}\right)$ & 0.186 & 3.654 \\
\hline$T / K$ & $293 \mathrm{~K}$ & 140 \\
\hline$R^{a} / R^{b}$ & $0.0698 / 0.1533$ & $0.0463 / 0.1363$ \\
\hline \multicolumn{3}{|c|}{${ }^{a} R=\Sigma|| F_{\mathrm{o}}|-| F_{\mathrm{c}}|| / \Sigma\left|F_{\mathrm{o}}\right| \cdot{ }^{b} R_{w}=\left[\Sigma\left[w\left(F_{o}{ }^{2}-F_{c}^{2}\right)^{2}\right] / \Sigma w\left(\mathrm{Fo}^{2}\right)^{2}\right]^{1 / 2}$} \\
\hline
\end{tabular}


Table S6 Crystal Date and Structure Refinements for $\mathbf{6}$ and $\mathbf{7}$

\begin{tabular}{|c|c|c|}
\hline & 6 & 7 \\
\hline Chemical formula & $\mathrm{C}_{23} \mathrm{H}_{20} \mathrm{FNO}_{3} \mathrm{~S}$ & $\mathrm{C}_{24} \mathrm{H}_{23} \mathrm{FN}_{2} \mathrm{O}_{4} \mathrm{~S}$ \\
\hline Formula weight & 409.46 & 454.50 \\
\hline Crystal system & monoclinic & triclinic \\
\hline Space group & P $121 / \mathrm{n} 1$ & $P-1$ \\
\hline$a(\AA)$ & $10.6561(9)$ & $9.6710(8)$ \\
\hline$b(\AA)$ & $10.8386(8)$ & $9.8852(8)$ \\
\hline$c(\AA)$ & $17.3501(13)$ & $12.2363(10)$ \\
\hline$V\left(\AA^{3}\right)$ & $2002.5(3)$ & $1113.66(16)$ \\
\hline$\alpha\left(^{\circ}\right)$ & 90 & $94.891(7)$ \\
\hline$\beta\left(^{\circ}\right)$ & $92.099(7)$ & $101.174(7)$ \\
\hline$\gamma\left(\left(^{\circ}\right)\right.$ & 90 & $101.834(7)$ \\
\hline$Z$ & 4 & 2 \\
\hline $\mathrm{F}(000)$ & 856.0 & 476.0 \\
\hline$G O F$ & 0.966 & 1.042 \\
\hline$D / \mathrm{g} \mathrm{cm}^{-3}$ & 1.358 & 1.355 \\
\hline$\mu\left(\mathrm{mm}^{-1}\right)$ & 0.195 & 1.653 \\
\hline$T / K$ & 293 & 293 \\
\hline$R^{a} / R^{b}$ & $0.0544 / 0.1832$ & $0.0841 / 0.2562$ \\
\hline
\end{tabular}


4. X-ray Crystal Structures (30\% thermal ellipsoid probability levels)
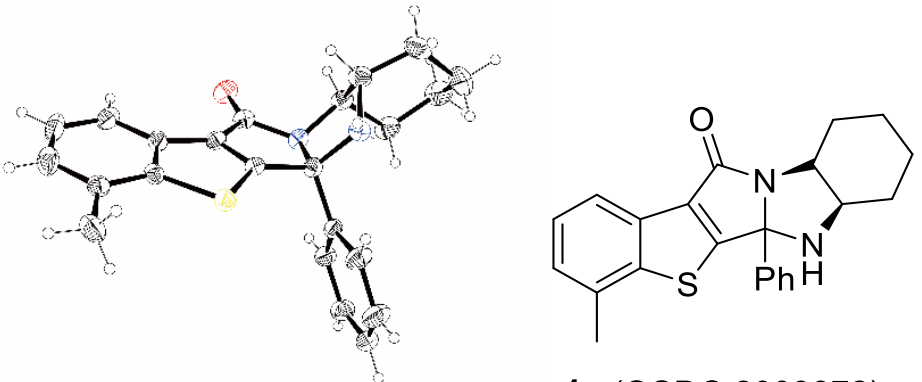

4p (CCDC 2003872)
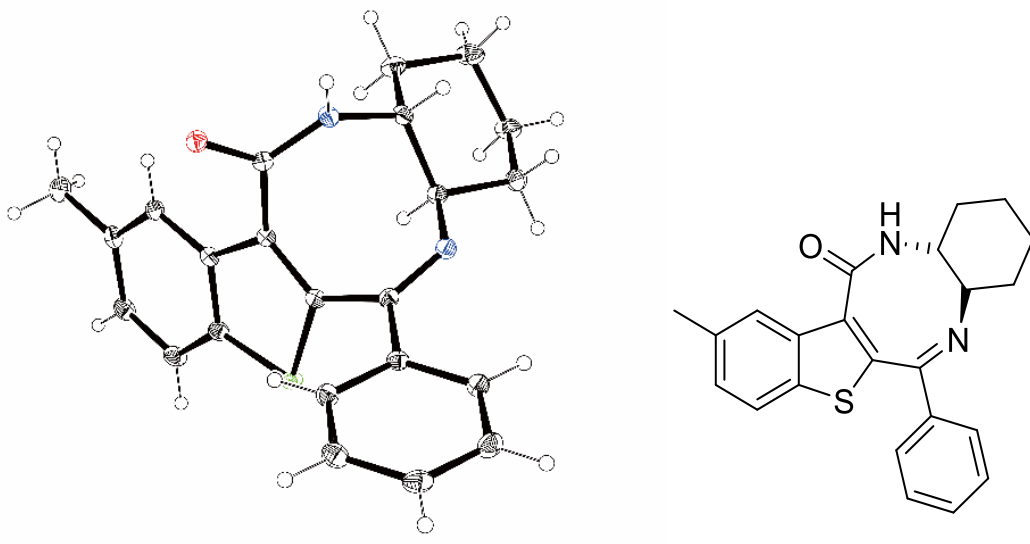

$5 a(C C D C 2003873)$
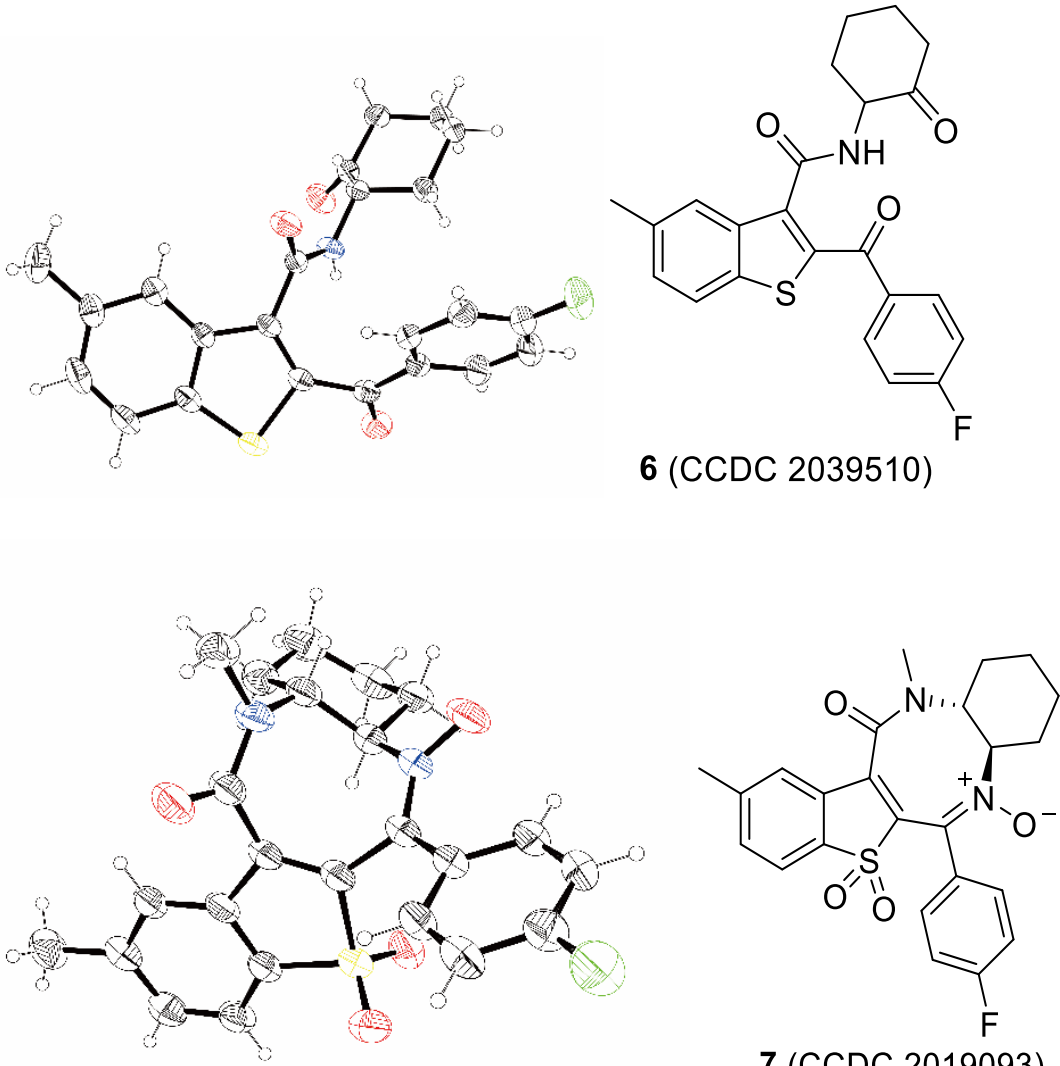

7 (CCDC 2019093) 


\section{References}

(1) Frisch, M. J.; Trucks, G. W.; Schlegel, H. B.; Scuseria, G. E.; Robb, M. A.; Cheeseman, J. R.; Scalmani, G.; Barone, V.; Petersson, G. A.; Nakatsuji, H.; Li, X.; Caricato, M.; Marenich, A. V.; Bloino, J.; Janesko, B. G.; Gomperts, R.; Mennucci, B.; Hratchian, H. P.; Ortiz, J. V.; Izmaylov, A. F.; Sonnenberg, J. L.; Williams-Young, D.; Ding, F.; Lipparini, F.; Egidi, F.; Goings, J.; Peng, B.; Petrone, A.; Henderson, T.; Ranasinghe, D.; Zakrzewski, V. G.; Gao, J.; Rega, N.; Zheng, G.; Liang, W.; Hada, M.; Ehara, M.; Toyota, K.; Fukuda, R.; Hasegawa, J.; Ishida, M.; Nakajima, T.; Honda, Y.; Kitao, O.; Nakai, H.; Vreven, T.; Throssell, K.; Montgomery, J. A.; Peralta, Jr., J. E.; Ogliaro, F.; Bearpark, M. J.; Heyd, J. J.; Brothers, E. N.; Kudin, K. N.; Staroverov, V. N.; Keith, T. A.; Kobayashi, R.; Normand, J.; Raghavachari, K.; Rendell, A. P.; Burant, J. C.; Iyengar, S. S.; Tomasi, J.; Cossi, M.; Millam, J. M.; Klene, M.; Adamo, C.; Cammi, R.; Ochterski, J. W.; Martin, R. L.; Morokuma, K.; Farkas, O.; Foresman, J. B.; Fox, D. J. Gaussian 16, Revision C.01, Gaussian, Inc., Wallingford CT, 2016.

(2) Becke, A. D. Density-functional thermochemistry. III. The role of exact exchange. J. Chem. Phys. 1993, $98,5648-5652$.

(3) Scalmani, G.; Frisch, M. J. Continuous surface charge polarizable continuum models of solvation. I. General formalism. J. Chem. Phys. 2010, 132, 114110-114124.

(4) Zhao, Y.; Truhlar, D. G. A new local density functional for main-group thermochemistry, transition metal bonding, thermochemical kinetics, and noncovalent interactions. J. Chem. Phys. 2006, 125, 194101-194118.

(5) a) Huang, F.; Lu, G.; Zhao, L.; Li, H.; Wang, Z.-X. The Catalytic Role of N-Heterocyclic Carbene in a Metal-Free Conversion of Carbon Dioxide into Methanol: A Computational Mechanism Study. J. Am. Chem. Soc. 2010, 132, 12388-12396; b) Plata, R. E.; Singleton, D. A. A Case Study of the Mechanism of Alcohol-Mediated Morita Baylis-Hillman Reactions. The Importance of Experimental Observations. J. Am. Chem. Soc. 2015, 137, 3811-3826; c) Kua, J.; Rodriguez, A. A.; Marucci, L. A.; Galloway, M. M.; De Haan, D. O. Free Energy Map for the Co-Oligomerization of Formaldehyde and Ammonia. J. Phys. Chem. A 2015, 119, 2122-2131. 